\title{
EVALUATION OF ENDOCRANIAL BONY CHANGES IN RELATION TO TUBERCULOSIS IN THE ROBERT J. TERRY ANATOMICAL SKELETAL COLLECTION (WASHINGTON, DC, USA)
}

\section{By: Olga SPEKKER}

Supervisors:

Erika MOLNÁR, PhD

ASSISTANT PROFESSOR

\section{György PÁLFI, PhD}

ASSOCIATE PROFESSOR \& HEAD OF DEPARTMENT

DOCTORAL SCHOOL OF BIOLOGY



DEPARTMENT OF BIOLOGICAL ANTHROPOLOGY FACULTY OF SCIENCE AND INFORMATICS UNIVERSITY OF SZEGED

SZEGED 
To my beloved grandfather, in memoriam.

-2 - 
"We do not know a truth without knowing its cause."

-Aristotle 


\section{TABLE OF CONTENTS}

TITLE PAGE



EPIGRAPH









1.1 Aetiological agents, transmission, and pathogenesis of tuberculosis ...................................10

1.2 Palaeoepidemiological and epidemiological aspects of tuberculosis ....................................11

1.3 Palaeopathological diagnosis of tuberculosis - Non-endocranial bony changes ..................12

1.4 Palaeopathological diagnosis of tuberculosis - Endocranial bony changes .........................25













4.1.1 Abnormally pronounced digital impressions.................................................................. 48



4.1.3 Abnormal blood vessel impressions.............................................................................6



4.2 Co-occurrence of endocranial alterations probably associated with tuberculosis ................86

4.3 Association of endocranial and non-endocranial lesions likely related to tuberculosis ........95



6 RESEARCH SIGNIFICANCE \& PERSPECTIVES ............................................ 128
















\section{LIST OF ABBREVIATIONS}

ABVI: $\quad$ abnormal blood vessel impression

AIDS: acquired immune deficiency syndrome

APDI: abnormally pronounced digital impression

BCG: $\quad$ Bacillus Calmette-Guérin vaccine

CNS: $\quad$ central nervous system

CSF: $\quad$ cerebrospinal fluid

DI: digital impression

eICP: $\quad$ elevated intracranial pressure

GI: $\quad$ granular impression

HIV: $\quad$ human immunodeficiency virus

HPO: hypertrophic pulmonary osteopathy

MDR-TB: multidrug-resistant tuberculosis

MTBC: $\quad$ Mycobacterium tuberculosis complex

NMNH: National Museum of Natural History

NTB: non-tuberculous

PA: periosteal apposition

PNBF: periosteal new bone formation

RR-TB: $\quad$ rifampicin-resistant tuberculosis

SES: $\quad$ serpens endocrania symmetrica

TB: $\quad$ tuberculosis/tuberculous

TBM: $\quad$ tuberculous meningitis

WHO: World Health Organization 


\section{LIST OF FIGURES \& TABLES \\ (containing shortened titles)}

\section{FIGURES}

Figure 1: TB osteolytic lesions in the vertebral body

Figure 2: Complications of spinal TB: Pott's gibbus and signs of an overlying cold abscess

Figure 3: Vertebral hypervascularisation affecting the thoracic bodies

Figure 4: Lytic lesions representing TB osteomyelitis of the clavicle.

Figure 5: TB arthritis of the knee joint

Figure 6: PNBFs referring to pulmonary TB and/or TB pleurisy on the visceral surface of ribs.

Figure 7: PNBFs indicative of HPO on the diaphysis of long tubular bones .24

Figure 8: APDIs suggestive of eICP on the endocranial surface of the frontal bone.....

Figure 9: ABVIs and PAs on the frontal bone 30

Figure 10: ABVIs and PAs on the frontal bone (thin-ground sections viewed in polarised light) ................31



Figure 12: GIs accompanied by ABVIs representing TBM on the inner surface of the parietal bone ............33





Figure 15: Selection, moving, and evaluation of skeletons in the Terry Collection .....................................40

Figure 16: Demographic profile of specimens surveyed in the TB group............................................41

Figure 17: Demographic profile of specimens surveyed in the NTB group ..........................................41

Figure 18: Different types of endocranial bony alterations probably related to TBM...............................42

Figure 19: Different stages of multifocal PAs and ABVIs on the endocranial surface of the frontal bone.....44

Figure 20: Different stages of multifocal GIs on the inner surface of the occipital bone...........................45

Figure 21: Different stages of the prominence of APDIs on the endocranial surface of the frontal bone.......46

Figure 22: Demographic profile of specimens exhibiting APDIs in the TB group ..................................49

Figure 23: Demographic profile of specimens exhibiting APDIs in the NTB group .................................50

Figure 24: Different stages of the prominence of APDIs on the inner surface of the skull........................51

Figure 25: Distribution of specimens affected by APDIs by stages of the prominence ..............................52

Figure 26: Shallow APDIs on the endocranial surface of the frontal bone (Terry No. 30R) ......................53

Figure 27: Moderately deep APDIs on the inner surface of the frontal bone (Terry No. 382R) ..................54 
Figure 28: Multifocal PAs and ABVIs in the APDIs on the frontal bone (Terry No. 1033)

Figure 29: Demographic profile of specimens exhibiting PAs in the TB group

Figure 30: Demographic profile of specimens exhibiting PAs in the NTB group .58

Figure 31: Typical localisations of PAs on the endocranial surface of the skullcap and skull base .59

Figure 32: Distribution of specimens affected by PAs by no. of concomitantly involved cranial bones 60

Figure 33: Multifocal ABVIs accompanied by PAs on the occipital bone (Terry No. 987)

Figure 34: Multifocal GIs accompanied by PAs on the temporal bone (Terry No. 1027) ...... .63

Figure 35: Multifocal ABVIs and PAs on the frontal and parietal bones (Terry No. 1027)

Figure 36: Multifocal PAs on the endocranial surface of the frontal bone (Terry No. 1300) ...... .65

Figure 37: Demographic profile of specimens exhibiting ABVIs in the TB group..... .67

Figure 38: Demographic profile of specimens exhibiting ABVIs in the NTB group..... .68

Figure 39: Typical localisations of ABVIs on the inner surface of the skullcap and skull base

Figure 40: Distribution of specimens affected by ABVIs by no. of concurrently involved cranial bones ......70

Figure 41: Multifocal ABVIs accompanied by PAs on the parietal bone (Terry No. 254) .72

Figure 42: ABVIs on the sphenoid and parietal bones (Terry No. 1030). .73

Figure 43: ABVIs on the endocranial surface of the occipital bone (Terry No. 1030). .74

Figure 44: ABVIs on the inner surface of the frontal and parietal bones (Terry No. 1555). .75

Figure 45: Demographic profile of specimens exhibiting GIs in the TB group .77

Figure 46: Demographic profile of specimens exhibiting GIs in the NTB group ...... .78

Figure 47: Typical localisations of GIs on the endocranial surface of the skull base..... .79

Figure 48: Distribution of specimens affected by GIs by no. of simultaneously involved cranial bones........80

Figure 49: GIs on the endocranial surface of the frontal, parietal, and temporal bones (Terry No. 522).......82

Figure 50: GIs on the inner surface of the frontal and occipital bones (Terry No. 562) .83

Figure 51: Unifocal GIs on the endocranial surface of the occipital and sphenoid bones (Terry No. 566).....84

Figure 52: Multifocal GIs on the inner surface of the frontal bone (Terry No. 933R) .85

Figure 53: Demographic profile of specimens with association of endocranial lesions in the TB group.......87

Figure 54: Demographic profile of specimens with association of endocranial lesions in the NTB group.....88

Figure 55: Distribution of specimens by no. of presented probable TBM-related endocranial lesion types ...89

Figure 56: Multifocal ABVIs and PAs in the APDIs on the frontal bone (Terry No. 304)

Figure 57: Multifocal PAs and GIs in the APDIs on the frontal bone (Terry No. 1159) .91 
Figure 58: Multifocal GIs and PAs on the endocranial surface of the occipital bone (Terry No. 1222) .........92

Figure 59: Multifocal ABVIs covered by PAs on the inner surface of the parietal bone (Terry No. 1222)....93

Figure 60: Multifocal ABVIs and PAs on the occipital and temporal bones (Terry No. 1322) ....................94

Figure 61: Demographic profile of specimens with association of endo- and non-endocranial lesions in the TB group.........96

Figure 62: Demographic profile of specimens with association of endo- and non-endocranial lesions in the NTB group ......97

Figure 63: Distribution of specimens by no. of presented probable TB-related non-endocranial lesion types.......98

Figure 64: No. of specimens exhibiting different types of TB-related non-endocranial bony changes..........99

Figure 65: Slight PNBFs on the visceral surface of the vertebral end of a rib (Terry No. 468) ...................100

Figure 66: Cortical erosion and PNBFs on the thoracic vertebral bodies (Terry No. 468) .........................101

Figure 67: Erosive lesions and bony bridges on the lumbar vertebral bodies (Terry No. 468) ....................102

Figure 68: TB involvement of the sacroiliac joint and pubic symphysis (Terry No. 468) .........................103

Figure 69: ABVIs on the endocranial surface of the frontal and parietal bones (Terry No. 902) ................104

Figure 70: TB arthritis of the atlanto-occipital and facet joints (Terry No. 902) ......................................105

Figure 71: Superficial vertebral changes on the thoracic and lumbar vertebrae (Terry No. 902) ...............106

Figure 72: TB osteomyelitis of the body of the sternum (Terry No. 902) ...............................................107

Figure 73: Small erosive lesion in the costal groove of a rib (Terry No. 902) .......................................108

Figure 74: Slight PNBFs on the medial surface of the mid-shaft of the tibia (Terry No. 902)....................108

Figure 75: Slight erosion on the articular surfaces of the sacroiliac joint (Terry No. 902) .......................109

Figure 76: Typical localisations of PAs, ABVIs, and GIs on the endocranial surface of the skull 123

\section{TABLES}

Table 1: Data collection sheet for recording endocranial alteration types probably related to TBM 43

Table 2: No. of specimens showing APDIs in the TB group and NTB group by sex................................48

Table 3: No. of specimens exhibiting PAs in the TB group and NTB group by sex .................................56

Table 4: Distribution of specimens revealing PAs by affected cranial bones, extent, and no. of lesions ........61

Table 5: No. of specimens exhibiting ABVIs in the TB group and NTB group by sex .66

Table 6: Distribution of specimens showing ABVIs by affected cranial bones, extent, and no. of lesions.....71

Table 7: No. of specimens exhibiting GIs in the TB group and NTB group by sex 76

Table 8: Distribution of specimens revealing GIs by affected cranial bones, extent, and no. of lesions .........81

Table 9: No. of specimens showing association of endocranial bony alterations by sex. .86

Table 10: No. of specimens exhibiting association of endo- and non-endocranial bony changes by sex .95 
1 INTRODUCTION

$-9-$ 


\subsection{Aetiological agents, transmission, and pathogenesis of tuberculosis}

Tuberculosis (TB) is an airborne infectious disease of both humans and animals that is caused by a number of pathogenic mycobacterial species belonging to the Mycobacterium tuberculosis complex (MTBC) (i.e., M. tuberculosis, M. africanum, M. canettii, M. bovis, M. caprae, M. microti, M. pinnipedii, M. orygis, M. mungi, M. suricattae, "dassie bacillus", and "chimpanzee bacillus"), with M. tuberculosis being the most common cause of TB in humans. TB bacteria enter the human body usually via the respiratory tract through inhalation of droplets expelled into the air by an individual with active pulmonary TB disease during sneezing, coughing or talking. Following inhalation, pathogens that reach the alveoli in the lungs may be eliminated by the host's immune system (i.e., rapid clearance) (Flynn \& Chan, 2001; Flynn et al., 2011; Gideon \& Flynn, 2011; Ernst, 2012; Gagneux, 2012; Gengenbacher \& Kaufmann, 2012; Miranda et al., 2012; Bañuls et al., 2015; Brites \& Gagneux, 2015; Fogel, 2015; Getahun et al., 2015; Clarke et al., 2016; Pai et al., 2016).

However, in most people, TB bacteria are able to escape elimination in the alveoli and invade into the lung parenchyma, where their presence triggers the recruitment of an increasing number of immune cells (e.g., macrophages, dendritic cells, and lymphocytes) to sites of infection, ultimately resulting in the formation of tuberculous granulomas, also known as tubercles. On the one hand, tubercles provide an isolated microenvironment in which host cells interact to control and prevent dissemination of the infection; whereas on the other hand, they function as a survival niche in which TB bacteria can replicate or persist in a dormant state within the lung tissue until opportunity arises for them to reactivate and spread. In the minority of people affected $(\sim 10 \%)$ - mainly in infants and children -, tubercles fail to contain the infection and TB bacteria can disseminate throughout the lung or into other parts of the body; thus, the infection progresses into primary active TB disease (i.e., the infected person becomes symptomatic and contagious), usually within the first 2 years after exposure. Nevertheless, in most cases ( 90\%) - defined as latent TB infection (i.e., the infected person is asymptomatic and not contagious) -, TB bacteria remain dormant within the tubercles for a long time (even for a lifetime), with subsequent reactivation occurring only when certain factors (e.g., malnutrition, HIV infection, and diabetes mellitus) compromise the host's immune system (Flynn \& Chan, 2001; Flynn et al., 2011; Gideon \& Flynn, 2011; Ernst, 2012; Gengenbacher \& Kaufmann, 2012; Miranda et al., 2012; Fogel, 2015; Getahun et al., 2015; Pai et al., 2016). 
Although tuberculosis primarily affects the lungs (i.e., pulmonary TB), the haematogenous or lymphogenous spread of TB bacteria to other parts of the body, including the skeleton or the central nervous system (CNS), results in the development of different types of extra-pulmonary tuberculosis (e.g., miliary TB, skeletal TB, and CNS TB) that together constitute about 15 to $20 \%$ of all cases with active tuberculosis (Golden \& Vikram, 2005; Vanhoenacker et al., 2009; Mohapatra et al., 2011; Fuentes Ferrer et al., 2012; Pai et al., 2016; Held et al., 2017).

\subsection{Palaeoepidemiological and epidemiological aspects of tuberculosis}

Tuberculosis is one of the oldest known infectious diseases that has been plaguing mankind for thousands of years: the earliest recognised and verified TB cases (e.g., Nicklisch et al., 2012; Spekker et al., 2012; Köhler et al., 2014, 2016; Baker et al., 2015; Hershkovitz et al., 2015; Masson et al., 2015; Sparacello et al., 2017) come from the Neolithic period. Apparently, TB remained relatively sporadic until the 1700's but - as a consequence of increased population density and unsanitary living conditions - started to reach epidemic levels during the Industrial Revolution. From the second half of the $19^{\text {th }}$ century, a number of factors (such as the general improvement in living conditions, sanitation, and nutrition) have been attributed to a reduction in the number of TB cases in developed countries. Later, the decline in TB incidence rates was even more accelerated by the introduction of the Bacillus Calmette-Guérin (BCG) vaccine and the use of antibiotics (e.g., streptomycin, isoniazid, and rifampicin). TB epidemic trends in Hungary followed those of Western Europe, with an approximately 75-year delay. The steady decrease in TB incidence in developed countries has led to predictions of the complete eradication of the disease by the end of the $20^{\text {th }}$ century. Nonetheless, particularly fuelled by the growing human immunodeficiency virus/acquired immune deficiency syndrome (HIV/AIDS) pandemic and the emergence of multidrug-resistant tuberculosis (MDR-TB), TB incidence started to increase again in the late 1980's. In 1993 (WHO, 1994), tuberculosis has been declared a global health threat by the World Health Organization (WHO) (Friend \& Watson, 1998; Hutás, 1999; Pálfi et al., 1999, 2015; Zumla et al., 2000; Roberts \& Buikstra, 2003; Smith, 2003; Golden \& Vikram, 2005; Gagneux, 2012; Galagan, 2014; Bañuls et al., 2015; David et al., 2015; Fogel, 2015; Pai et al., 2016).

In this day and age, more than 2 billion people - about one-third of the total population of the world - are latently infected with the causative agent of TB, of whom only 5 to $15 \%$ are at risk of eventually developing active TB disease during their lifetime (Golden \& 
Vikram, 2005; Flynn et al., 2011; Gengenbacher \& Kaufmann, 2012; Miranda et al., 2012; Getahun et al., 2015; Esteves et al., 2017; WHO, 2017). According to estimates of the WHO (2017), in 2016, there were approximately 10.4 million new incident cases of active TB disease, of which about $10 \%$ were among HIV-positive people. Tuberculosis remained one of the top 10 causes of death and the leading cause of death from a single infectious agent (ranking above HIV/AIDS), with accounting for 1.3 million deaths among HIV-negative individuals and for an additional 374,000 TB-related deaths among people co-infected with HIV. Drug resistance surveillance data show that in 2016, there were an estimated 490,000 new incident cases of MDR-TB worldwide, of which $6.2 \%$ were extensively drug-resistant. An additional 110,000 people were susceptible to isoniazid but resistant to rifampicin (RRTB), and a total of 240,000 deaths occurred due to MDR/RR-TB globally. Tuberculosis has been reported from all parts of the world but the majority of new incident cases and deaths (more than 95\%) occurred in low-income and middle-income countries. In 2016, the most affected WHO region was the South-East Asia Region ( $45 \%)$, followed by the African ( 25\%) and Western Pacific Regions ( 17\%). Seven countries comprised about two-thirds of new incident cases (in descending order): India, Indonesia, China, the Philippines, Pakistan, Nigeria, and South Africa.

The global health emergency presented by TB today has sparked a renewed interest and funding to the research of the disease and of its aetiological agents, including science projects concerning the origin and evolutionary history of the MTBC, as well as the palaeopathological diagnostics for TB (Pálfi et al., 2015; Pai et al., 2016; WHO, 2017).

\subsection{Palaeopathological diagnosis of tuberculosis - Non-endocranial bony changes}

Traditionally, the diagnosis of TB in ancient human bone remains relies on the identification of pathological lesions in the skeleton that are related to different forms of TB, such as pulmonary TB, osteoarticular TB, and CNS TB (Kelley \& Micozzi, 1984; Aufderheide \& Rodríguez-Martín, 1998; Ortner, 2003, 2008; Santos \& Roberts, 2006; Pálfi et al., 2012; Mariotti et al., 2015).

Nowadays, skeletal tuberculosis occurs in less than $2 \%$ of all active TB cases and accounts for 10 to $35 \%$ of extra-pulmonary TB cases. Nevertheless, over the past two decades, the incidence of osteoarticular TB has increased worldwide, especially in lowincome and middle-income countries, due in part to the HIV/AIDS pandemic. In high-TBburden countries, skeletal TB affects more commonly children and young adults, mainly during the first three decades of life; whereas in low-TB-burden countries, the majority of 
osteoarticular TB cases occur mainly in foreign-born younger adults (particularly between 20 to 30 years of age) from TB-endemic regions of the world or in native-born older adults (predominantly between 60 to 70 years of age). Similar to other forms of TB, predisposing factors for tuberculous involvement of the bones and/or joints include factors contributing to impairment of the host's immune system, such as HIV infection, immunosuppressive therapy, and alcohol or substance abuse (Houshian et al., 2000; Jutte et al., 2004; Golden \& Vikram, 2005; Kumar, 2005; Sandher et al., 2007; Vanhoenacker et al., 2009; Garg \& Somvanshi, 2011; Fuentes Ferrer et al., 2012; Rasouli et al., 2012; Pigrau-Serrallach \& Rodríguez-Pardo, 2013; Patel et al., 2016; De la Garza Ramos et al., 2017; Esteves et al., 2017; Held et al., 2017).

Osteoarticular tuberculosis is usually secondary to haematogenous spread of TB bacteria from a primary location outside the skeleton (such as the respiratory, alimentary or genitourinary system) into the bone and/or synovial tissue during or shortly after the bacteraemic stage of primary infection or late reactivation of the disease; however, in the minority of TB cases, lymphogenous dissemination, contiguous spread from adjacent structures or direct inoculation of pathogens into a skeletal site can also occur. Any part of the skeleton can be affected by TB but areas containing large amounts of red bone marrow are most frequently predisposed to be involved because of the rich blood supply of the marrow; thus, the most preferential skeletal localisation of TB is the spine $(50-70 \%)$, followed by the hip and knee joints (10-15\% each). Deposition of TB bacteria into the skeleton can lead to the development of three main forms of osteoarticular TB: spinal tuberculosis or tuberculous spondylitis (i.e., combination of tuberculous vertebral osteomyelitis and arthritis), tuberculous osteomyelitis of the extra-spinal bones, and tuberculous arthritis of the extra-spinal joints, with the first being the most common type ( 50\%) (Aufderheide \& Rodríguez-Martín, 1998; Lagier, 1999; Ortner, 2003; Golden \& Vikram, 2005; Kumar, 2005; Spiegel et al., 2005; Vanhoenacker et al., 2009; Agrawal et al., 2010; Garg \& Somvanshi, 2011; Fuentes Ferrer et al., 2012; Rasouli et al., 2012; PigrauSerrallach \& Rodríguez-Pardo, 2013; Patel et al., 2016; De la Garza Ramos et al., 2017; Esteves et al., 2017).

Tuberculous spondylitis - also known as spondylitis tuberculosa or Pott's disease in honour of English surgeon Sir Percivall Pott (1779) who first described this clinical entity in the modern era - is characterised by an insidious onset and a slowly progressive course, with disease duration generally varying from a few months to a few years. Any part of the vertebral column can be involved by tuberculosis but the most frequently affected regions 
are the lower thoracic (40-50\%) and upper lumbar (35-40\%) spine; whereas the cervical region is involved in only approximately $10 \%$ of all cases with tuberculous spondylitis. Although spinal TB typically affects two or more contiguous vertebrae, multilevel noncontiguous involvement or solitary vertebral lesions may also occur in some patients. Based on the localisation pattern of tuberculous alterations in the vertebra, five main types of tuberculous spondylitis can be distinguished, namely the paradiscal, central, anterior subligamentous, posterior, and articular forms (Ortner, 2003; Kumar, 2005; Spiegel et al., 2005; Sridhar \& Krishnan, 2009; Agrawal et al., 2010; Garg \& Somvanshi, 2011; Fuentes Ferrer et al., 2012; Rasouli et al., 2012; Pigrau-Serrallach \& Rodríguez-Pardo, 2013; Acharya \& Gibbs, 2016; Patel et al., 2016; De la Garza Ramos et al., 2017; Esteves et al., 2017).

The most common type of spinal TB, namely the paradiscal form, accounts for approximately 90 to $95 \%$ of all cases with tuberculous spondylitis. It arises from the anterior subchondral region of the vertebral body (adjacent to the superior or inferior cartilaginous endplates) that has a dense end-arteriolar network making it susceptible to bacterial seeding via the segmental arterial circulation (anterograde blood flow). Lodgement of TB bacteria into the subchondral region triggers the onset of granulomatous inflammation of the cancellous bone with tubercle formation in the red bone marrow, followed by secondary involvement of the bone trabeculae. Development and caseous necrosis of the gradually expanding and coalescing tubercles located in the marrow induce growth of the initial intravertebral abscess and establishment of additional intra-vertebral abscesses within the affected vertebral body, as well as deprivation of the blood supply to cancellous bone due to TB involvement of segmental artery branches with consequent thrombosis and arterial occlusion. Any or all of the aforementioned processes result in necrosis and resorption of bone trabeculae that ultimately lead to the formation of osteolytic lesions in the subchondral region (Fig. 1A-B), with subsequent involvement of the entire vertebral body and occasionally of the posterior vertebral elements. Later, the intra-vertebral abscess extends towards the anterior and/or posterior longitudinal ligaments that - at least temporarily resist the horizontal progression of its passage; thus, TB infection spreads vertically (upwards or downwards) beneath the ligaments from the primarily affected vertebra into a similar location at one or more contiguous vertebrae or beyond, with relative sparing of the adjoining intervertebral discs until more advanced stages of the disease, most likely due to lack of proteolytic enzymes in TB bacteria (Aufderheide \& Rodríguez-Martín, 1998; Ortner, 
2003; Kumar, 2005; Spiegel et al., 2005; Agrawal et al., 2010; Garg \& Somvanshi, 2011; Rasouli et al., 2012; Acharya \& Gibbs, 2016; Esteves et al., 2017).

A)



B)



Figure 1: TB osteolytic lesions affecting the upper half of the $L 2$ body: A) superior view and $B$ ) left antero-lateral view (Bácsalmás - Homokbánya, Hungary, $16^{\text {th }}-17^{\text {th }}$ century AD, Grave No. 115, Juvenis, male) (Maczel, 2003).

Nevertheless, progressive destruction of the subchondral region of two adjacent vertebral bodies may compromise nutrition of the intervertebral disc abutted by them that can lead to its degeneration or result in its herniation into the weakened adjoining vertebral bodies, with gradual diminution or eventual loss of the intervertebral disc space. Degenerated and/or herniated discs are more prone to seeding by TB bacteria from the subchondral cancellous bone either via subligamentous dissemination or contiguous spread through the cartilaginous endplate. In children, the intervertebral disc - because of its vascularised nature - can represent the initial site of tuberculous spondylitis; whereas in adults, the age-related avascularity of the intervertebral disc prevents it from serving as the primary focus of infection in the vertebral column; therefore, dissemination of TB bacteria from the infected intervertebral disc into the abutting vertebral bodies occurs scarcely (Aufderheide \& Rodríguez-Martín, 1998; Agrawal et al., 2010; Garg \& Somvanshi, 2011; Rasouli et al., 2012; Esteves et al., 2017).

The weakening of the affected vertebral bodies due to TB osteolytic lesions may result in their consequent collapse under the weight of the trunk that is characterised by a wedgeshaped appearance, since cavitation of the infected vertebrae predominantly involves their anterior part, with intact or less destroyed posterior vertebral elements. Depending on its location in the spine, the collapse of one or more contiguous vertebrae may lead to the 
development of different spinal deformity types, most frequently of a sharply angular kyphosis in the thoracic region (i.e., Pott's gibbus) with its severity particularly determined by the extent of vertebral destruction, the spinal region affected by TB osteolytic lesions, as well as the number of vertebrae involved by the pathological process (Fig. 2A). Nonetheless, vertebral collapse occurring in the lumbar spine may terminate with telescoping of the affected area. Progressive vertebral destruction and consequent spinal deformity may be associated with mechanical instability of the vertebral column that may result in subsequent bony fusion of the remnants of collapsed vertebral bodies, bony ankylosis of the facet joints, as well as formation of bony extensions interconnecting the adjacent vertebrae (i.e., bony bridges), in order to restabilise the spine. Destructive vertebral lesions very likely attributable to the paradiscal form of spinal TB were identified in a number of ancient human skeletons (e.g., Bartels, 1907; Marcsik, 1978; Bennike, 1999; Blondiaux et al., 1999; Gładykowska-Rzeczycka, 1999; Horáčková et al., 1999; Marcsik et al., 1999, 2009; Maczel, 2003; Suzuki \& Inoue, 2007; Ösz et al., 2009; Hajdu et al., 2012; Arrieta et al., 2014; Baker et al., 2015; Balázs et al., 2015; Kajdocsi Lovász, 2015; Molnár et al., 2015; Sparacello et al., 2017) and mummies (e.g., Allison et al., 1973; Nerlich et al., 1997; Strouhal, 1999; Lombardi \& Cáceres, 2000) from all parts of the world, and represent much of the evidence for tuberculosis in antiquity (Aufderheide \& Rodríguez-Martín, 1998; Palmer, 2002; Ortner, 2003; Agrawal et al., 2010; Jain, 2010; Garg \& Somvanshi, 2011; Rasouli et al., 2012; Esteves et al., 2017).

A)



B)



Figure 2: A) severe angular kyphosis (Pott's gibbus) involving the upper thoracic vertebrae (C7-T8) (Sükösd - Ságod, Hungary, $7^{\text {th }}-8^{\text {th }}$ century AD, Grave No. 19, Juvenis, female) (Marcsik et al., 1999) and $B$ ) probable traces of an overlying TB cold abscess on the anterior surface of the sacrum (Vésztó - Mágori-halom, $4^{\text {th }}-49^{\text {th }}$ century BC, Grave No. 6, Maturus, female) (Spekker et al., 2012). 
Formation of an extra-vertebral cold abscess or abscessus frigidus (i.e., a slowly progressive abscess without characteristic signs of inflammation, such as heat, erythema or tenderness that may become encapsulated and calcified over time) around osteolytic vertebral lesions is a common complication of tuberculous spondylitis - occurring in about two-thirds of all cases with spinal TB - that is secondary to the extension of infection from the affected area of the vertebral column into adjacent ligaments and soft tissues. The TB material may be accumulated within the prevertebral and/or paravertebral spaces with the formation of prevertebral (e.g., retropharyngeal abscess in the cervical region), as well as unilateral or bilateral paravertebral abscesses (e.g., psoas abscess in the thoracolumbar region), commonly associated with fistulae. The TB cold abscess may remain localised at the initial site of infection; however, in most cases, it extends vertically (usually downwards) beneath the anterior and/or posterior longitudinal ligaments or along the fascial planes. In response to an overlying TB cold abscess, erosive cortical bone destruction, as well as reactive new bone formations, may occur on the adjacent surfaces of bones (e.g., vertebrae, hip bones, and femora) (Fig. 2B). The above-mentioned bony changes and/or remains of TB cold abscesses were occasionally observed during the palaeopathological examination of ancient human skeletal and mummified remains dated to different archaeological periods (e.g., Dutour et al., 1999; Marcsik et al., 2009; Madkour, 2011a; Spekker et al., 2012; Kajdocsi Lovász, 2015; Molnár et al., 2015) (Aufderheide \& Rodríguez-Martín, 1998; Ortner, 2003; Garg \& Somvanshi, 2011; Rasouli et al., 2012; Acharya \& Gibbs, 2016; Ingole et al., 2016; Patel et al., 2016; Turliuc et al., 2016; Esteves et al., 2017).

Other types of spinal TB (e.g., central, anterior subligamentous, posterior, and articular forms) all together comprise approximately 5 to $10 \%$ of all cases with tuberculous spondylitis. The central type, resulting from haematogenous dissemination of TB bacteria via the Batson's paravertebral venous plexus into the spine (retrograde blood flow), arises from the mid-section of one or more distant or adjacent vertebral bodies instead of their subchondral region. Later, the infection extends centrifugally to involve the entire vertebral body without or with minimal involvement of the intervertebral disc. Destruction of the vertebral body by TB osteolytic lesions may lead to its subsequent ballooning and concentric collapse that resembles vertebra plana and indicates complete compression of the vertebral body under the weight of the trunk, with consequent development of spinal deformity (Kumar, 2005; Agrawal et al., 2010; Garg \& Somvanshi, 2011; Esteves et al., 2017).

The anterior subligamentous form of spinal TB is characterised by erosive cortical bone destruction mainly affecting the anterior and lateral surfaces of the involved vertebral 
bodies. It may be secondary to the development of an extra-vertebral TB abscess between the vertebral body and the anterior longitudinal ligament or periosteum that may extend vertically (upwards or downwards) over several contiguous vertebrae beneath the aforementioned fibrous structures. Subligamentous spread of the TB abscess results in stripping of the anterior longitudinal ligament and periosteum from the anterior and lateral vertebral surfaces that leads to deprivation of the periosteal blood supply to the vertebral body with consequent ischaemia. The combination of ischaemic and pressure effects caused by the presence and extension of the extra-vertebral TB abscess results in shallow erosion of the anterior and lateral surfaces of the affected vertebral bodies that produces a scalloped appearance (i.e., anterior "gouge defect" or "aneurysmal syndrome"). As the pathological process progresses, it may subsequently involve the anterior and lateral portions of the affected vertebral bodies, since avascular vertebrae are more susceptible to infection. Besides the erosive cortical bone destruction, interconnecting, stabilising bony extensions (i.e., bony bridges) may occur on the anterior and/or lateral aspects of the involved adjoining vertebral bodies that contribute to the preservation of the intervertebral disc spaces and prevention of the development of vertebral collapse and subsequent spinal deformity even in cases with severe bone loss (Sorrel \& Sorrel-Dejerine, 1932; Reid, 1949; Aufderheide \& Rodríguez-Martín, 1998; Palmer, 2002; Ortner, 2003; Kumar, 2005; Spiegel et al., 2005; Agrawal et al., 2010; Garg \& Somvanshi, 2011; Rivas-Garcia et al., 2013).

In one of the less frequent types of spinal TB (2-3\%), namely in the posterior or appendiceal form, the granulomatous inflammatory reaction is isolated to the posterior vertebral elements, without or with secondary involvement of the vertebral body. The disease may result from haematogenous spread of TB bacteria via the posterior external venous plexus (retrograde blood flow) or by direct inoculation of pathogens into the posterior vertebral elements (i.e., the pedicles and laminae of the vertebral arch, and the transverse and spinous processes). In posterior tuberculous spondylitis, the TB osteolytic lesions mainly affect only one vertebra, with pedicles and laminae representing the most common sites of involvement. Destruction of the posterior vertebral elements only is rarely accompanied by spinal deformity, since barely affecting the stability of the vertebral column; however, it is often associated with severe neurological deficit (e.g., paralysis), due in part to the compressive effect exerted by the TB material on the spinal cord or cauda equina (Aufderheide \& Rodríguez-Martín, 1998; Ortner, 2003; Sridhar \& Krishnan, 2009; Garg \& Somvanshi, 2011; Yazdi \& Rahimizadeh, 2012; Al-Khudaira \& Meir, 2014; Kumar, 2017). 
Similar to the posterior type, the articular or synovial form of spinal TB occurs in only the minority of patients with tuberculous spondylitis (e.g., the involvement of the atlantooccipital joint can be observed in less than $1 \%$ of all cases with spinal TB). It is usually secondary to haematogenous dissemination of TB bacteria through synovial vessels into the synovial membrane of the atlanto-occipital, atlanto-axial or facet joints (anterograde blood flow), with subsequent development of TB arthritis of the affected intervertebral joints (Ortner, 2003; Garg \& Somvanshi, 2011; Fuentes Ferrer et al., 2012; Qureshi et al., 2013; Mansukhani et al., 2014; Kumar, 2017).

In addition, circumferential, multiple, smooth-walled resorptive lesions (pits) often connected by horizontal vascular impressions on the anterior and lateral aspects of the thoracic (Fig. 3A-B) and lumbar vertebral bodies - representing hypervascularisation of the involved vertebrae - were described in several palaeopathological and palaeomicrobiological studies on osteoarchaeological series and documented skeletal collections (e.g., Ménard, 1888; Baker, 1999; Haas et al., 2000; Maczel, 2003; Giacon, 2008; Spekker et al., 2012; Baker et al., 2015; Guichón et al., 2015; Mariotti et al., 2015; Masson et al., 2015; Molnár et al., 2015) as probable but not specific signs of early-stage spinal TB.

A)



B)



Figure 3: Circumferential, multiple, smooth-walled pits connected by horizontal vascular impressions on the A) anterior and B) left lateral aspects of the T5-9 vertebrae (Vésztő - Mágori-halom, Hungary, $4^{t^{\text {th }}-49^{\text {th }}}$ century BC, Grave No. 33, Juvenis, male) (Spekker et al., 2012). 
In this day and age, extra-spinal TB osteomyelitis (Fig. 4A-B) is a rare medical condition, accounting for about 2 to $3 \%$ of all cases with osteoarticular TB. Any bone of the extra-spinal skeleton (e.g., sternum, ribs, as well as hip and cranial bones) can be affected by the disease but the metaphyses of short and long tubular bones are most frequently predisposed to be involved because of their rich blood supply. Similar to other forms of osteoarticular tuberculosis, extra-spinal $\mathrm{TB}$ osteomyelitis is generally secondary to haematogenous spread of TB bacteria from a primary location outside the skeleton into the bone. Lodgement of pathogens into the cancellous bone triggers the onset of its granulomatous inflammation with consequent formation of round or oval TB osteolytic lesions at sites of bacterial deposition. As the pathological process progresses, extra-osseous TB cold abscesses overlying the osteolytic lesions may develop that can be accompanied by fistulous perforation of the skin. Later, the infection may extend from the initial metaphyseal focus into the epiphysis, with subsequent TB arthritis of the adjacent joint. Although extraspinal TB osteomyelitis typically affects only one bone in the skeleton, simultaneous involvement of multiple bones may also occur in some patients, with the TB osteolytic lesions in different stages of development, as bacterial seeding of the affected bones may occur at different times during the course of the disease. Signs of extra-spinal TB osteomyelitis were occasionally identified in different bones - such as the cranial bones (e.g., Blondiaux et al., 1999; Strouhal, 1999; Dawson \& Brown, 2012; Colombo et al., 2015), tibia (e.g., Horáčková et al., 1999), humerus (e.g., Blondiaux et al., 1999) or clavicle (e.g., Spekker et al., 2012) - during the palaeopathological examination of ancient human skeletons and mummies (Aufderheide \& Rodríguez-Martín, 1998; Kam et al., 2000; Ortner, 2003; Spiegel et al., 2005; Vanhoenacker et al., 2009; Madkour, 2011b; Wiratnaya et al., 2017).

A)



B)



Figure 4: Smooth-walled lytic lesions on the A) acromial $(7 \times 8 \times 6 \mathrm{~mm})$ and B) sternal $(6 \times 7 \times 8 \mathrm{~mm})$ ends of the right clavicle, probably representing TB osteomyelitis (Vésztő - Mágori-halom, Hungary, $45^{\text {th }}-49^{\text {th }}$ century BC, Grave No. 6, Maturus, female) (Spekker et al., 2012). 
The second most common form of skeletal TB is tuberculous arthritis of the extraspinal joints, particularly of the large, weight-bearing joints, such as the hip (i.e., coxitis tuberculosa) and knee (i.e., gonitis tuberculosa) (Fig. 5A-B). It typically affects only one joint (i.e., monoarticular involvement) ( 90\%) and - similar to tuberculous spondylitis - has an insidious onset and a slowly progressive course. TB arthritis is usually secondary to seeding of TB bacteria into the synovial membrane of the joint capsule by haematogenous dissemination of pathogens through synovial vessels or into the subchondral bone by contiguous spread of microorganisms from an osteomyelitic focus in the metaphysis (more common in children) or epiphysis (more common in adults) of long tubular bones; therefore, the disease may begin in the synovial membrane (i.e., TB synovitis), in the subchondral bone or in both concomitantly. When TB arthritis starts as a synovitis, granulomatous inflammation of the synovial membrane results in synovial effusion and hypertrophy over the articular cartilage, as well as studding of the inner surface of the synovial membrane with tubercles. Invasion of the articular cartilage by the pannus initially formed at the joint periphery leads to progressive marginal erosion of the underlying articular cartilage and subsequently of the subchondral bone on both sides of the affected joint. Later, spreading of the pannus across the joint (from the periphery to the weight-bearing central areas) results in ultimate loss of the articular cartilage and further bone erosion, with gradual diminution and eventual obliteration of the articular cavity. Progressive destruction of the joint with consequent instability may be associated with the development of subluxation or dislocation. In more advanced stages of TB arthritis, reactive new bone formations may occur along the periarticular bone, and the disease may terminate in bony ankylosis of the affected joint. Extension of the infection into adjacent ligaments and soft tissues may result in the formation of cold abscesses with associated fistulae. When TB arthritis starts in the subchondral bone, the granulomatous inflammation of the cancellous bone results in the development of osteolytic lesions that may erode into the articular cavity, with subsequent TB involvement of the synovial membrane. Articular changes very likely attributable to tuberculosis were detected in extra-spinal weight-bearing (e.g., Blondiaux et al., 1999; Buzhilova et al., 1999; Gładykowska-Rzeczycka, 1999; Horáčková et al., 1999; Marcsik et al., 1999, 2009; Maczel, 2003; Ösz et al., 2009; Kajdocsi Lovász, 2015; Molnár et al., 2015; Paja et al., 2015; Cieślik, 2017) and non-weight-bearing (e.g., Bennike, 1999; Gładykowska-Rzeczycka, 1999; Maczel, 2003; Kajdocsi Lovász, 2015) joints in a number of ancient human remains dated to different archaeological periods (Aufderheide \& Rodríguez-Martín, 1998; Ortner, 2003; 
Spiegel et al., 2005; Vanhoenacker et al., 2009; Al-Sayyad \& Abumunaser, 2011; Mohapatra et al., 2011; Pigrau-Serrallach \& Rodríguez-Pardo, 2013; Tseng et al., 2014; Shapiro, 2015).

A)



B)



Figure 5: TB involvement of the right knee joint resulting in fusion of the adjoining surfaces of the right femur and tibia in a flexed position: $\mathrm{A}$ ) antero-medial view and B) lateral view (Bátmonostor Pusztafalu, Hungary, $12^{\text {th }}-16^{\text {th }}$ century AD, Grave No. 993, Senium, male) (photos by László Paja) (Paja et al., 2015).

Besides the above-mentioned lesions, periosteal new bone formations (PNBFs) affecting the visceral surface of ribs, as well as the shaft of short and long tubular bones, were associated with TB following examination of skeletons of known cause of death from modern identified skeletal collections (e.g., Hamann-Todd Human Osteological Collection, Robert J. Terry Anatomical Skeletal Collection, and Coimbra Identified Skeletal Collection), as they occurred more frequently in individuals reported to have died of TB than in specimens identified to have died of non-tuberculous (NTB) causes (e.g., Kelley \& Micozzi, 1984; Roberts et al., 1994; Winland et al., 1997; Rothschild \& Rothschild, 1998, 1999; Santos \& Roberts, 2001, 2006; Hershkovitz et al., 2002; Maczel, 2003; Matos \& Santos, 2006; Giacon, 2008; Mariotti et al., 2015).

PNBFs on the inner surface of ribs (Fig. 6) may very likely result from pulmonary TB that provokes a localised or generalised inflammatory response on the visceral costal surfaces by initiating the development of TB pleurisy with or without collection of pus in the pleural cavity (i.e., pleural empyema). PNBFs particularly involve ribs in the middle part of the thoracic cage $\left(4^{\text {th }}-8^{\text {th }}\right)$ - especially at their vertebral ends - , with the pattern and 
distribution of lesions resembling that of most often observed in the affected lung tissue during the pathogenesis of pulmonary TB. Localised PNBFs involving one or a few ribs may represent a focal TB pulmonary lesion subjacent to the pleura that leads to transpleural inflammation of ribs without pleural empyema; whereas generalised and multiple level PNBFs affecting many ribs may be indicative of a widespread costal inflammation with pleural empyema. Nevertheless, several pulmonary diseases other than TB, as well as nonpulmonary ones (e.g., acute lobar pneumonia, bronchiectasis, metastatic carcinoma, pyogenic osteomyelitis, syphilis, and actinomycosis), can also stimulate the formation of PNBFs on the inner surface of ribs (Roberts et al., 1994; Santos \& Roberts, 2001, 2006; Matos \& Santos, 2006; Mariotti et al., 2015).



Figure 6: PNBFs on the visceral surface of two rib fragments (Tápé - Széntéglaégető, Hungary, $12^{\text {th }}-13^{\text {th }}$ century BC, Grave No. 157, Maturus, male) (photo by László Paja) (Spekker et al., 2016).

Diffuse, bilateral, symmetrical PNBFs predominantly affecting the distal and periarticular parts of the shaft of short and long tubular bones (Fig. 7A-C) are characteristic features of hypertrophic pulmonary osteopathy (HPO). Although the aetiology of HPO is still unknown, the secondary form of the disease is frequently associated with pulmonary (e.g., pulmonary TB and lung cancer), as well as non-pulmonary (e.g., inflammatory bowel disease and cirrhosis) conditions. Similar to the previously discussed non-endocranial bony changes probably related to TB, signs of HPO were also observed during the palaeopathological examination of ancient human remains (e.g., Spekker et al., 2012; Masson et al., 2013, 2015; Baker et al., 2015; Hershkovitz et al., 2015; Anselmo et al., 2016) (Kelly et al., 1991; Assis et al., 2011; Yap et al., 2017).

Tuberculous involvement of the central nervous system (such as TB meningitis) may also result in the development of pathological bony changes, affecting the endocranial surface of the skull. 
Figure 7: PNBFs on the A) lateral surface of the diaphysis of the right tibia, B) anterior surface of the diaphysis of the right femur, and $\mathrm{C}$ ) postero-medial surface of the diaphysis of the left radius (Vésztö Mágori-halom, Hungary, $45^{\text {th }}-49^{\text {th }}$ century BC, Grave No. 6, Maturus, female) (Spekker et al., 2012).

A)



B)



C)






\subsection{Palaeopathological diagnosis of tuberculosis - Endocranial bony changes}

Nowadays, CNS TB - one of the most devastating clinical manifestations of tuberculosis - occurs in approximately $1 \%$ of all active TB cases and accounts for 5 to $15 \%$ of extra-pulmonary TB cases. TB may be exclusively confined to the CNS; however, concomitant active extra-neural forms of the disease - such as pulmonary $\mathrm{TB}$, miliary $\mathrm{TB}$ or spinal TB - may be present in about one-half of the patients with CNS TB. Furthermore, at autopsy, a large number of individuals died of pulmonary TB without developing neurological symptoms revealed tubercles in the CNS; therefore, indicating that involvement of the central nervous system in pulmonary TB is quite common (Arbeláez et al., 2004; Katti, 2004; Gauba \& Varma, 2005; Bill, 2006; Rock et al., 2008; Vinnard \& Macgregor, 2009; Cherian \& Thomas, 2011; Christensen et al., 2011; Marx \& Chan, 2011; Bini \& Hernández Pando, 2014; Daniele, 2014).

Usually, tuberculosis of the CNS results from haematogenous dissemination of TB bacteria from a primary location outside the CNS (such as the lungs or the gastrointestinal tract) and is characterised by a slowly progressive granulomatous inflammatory reaction that may affect the meninges, or the brain or spinal cord parenchyma. CNS TB develops in two stages. The initial stage involves the formation of small $(0.5-2 \mathrm{~mm})$ tubercles - also known as Rich foci - around TB bacteria deposited in the CNS via blood circulation during or shortly after the bacteraemic stage of primary infection or late reactivation of tuberculosis elsewhere in the body. Following their establishment, Rich foci may remain dormant for many years. Later, the enlargement or rupture of one or more Rich foci results in the development of different types of CNS TB (e.g., tuberculous meningitis, tuberculomas, and abscesses) (Rich \& McCordock, 1933; Garg, 1999, 2010; Bernaerts et al., 2003; Khoo et al., 2003; Arbeláez et al., 2004; Katti, 2004; Gauba \& Varma, 2005; Bill, 2006; Myers, 2007; Rock et al., 2008; Be et al., 2009; Vinnard \& Macgregor, 2009; Bano et al., 2012; Brancusi et al., 2012; Bini \& Hernández Pando, 2014; Daniele, 2014; Chaudhary et al., 2017).

The most common form of TB involvement of the CNS is tuberculous meningitis (TBM) of the leptomeninges (i.e., the pia and arachnoid mater), also known as leptomeningitis tuberculosa, accounting for about 70 to $80 \%$ of all cases with CNS TB. One of the most important risk factors for TBM is age. In low-income and middle-income countries with a high incidence of tuberculosis, children under the age of 5 years represent the most vulnerable group affected by the disease, usually developing TBM within 3 to 6 months of primary infection. However, in high-income countries with a low incidence of 
tuberculosis, TBM occurs predominantly in adults, particularly in immigrants from TBendemic regions of the world and in HIV-positive people, who are five times more likely to develop the disease than HIV-negative individuals. In adults, TBM usually results from the reactivation of dormant Rich foci, often many years after the primary infection. Besides age and HIV-infection, other predisposing factors for TBM include malnutrition, alcoholism, diabetes mellitus, and recent use of immunosuppressive drugs (such as corticosteroids) (Garg, 1999, 2010; Bernaerts et al., 2003; Katti, 2004; Gauba \& Varma, 2005; Bill, 2006; Myers, 2007; Rock et al., 2008; Thwaites et al., 2009; Cherian \& Thomas, 2011; Christensen et al., 2011; Bano et al., 2012; Thwaites, 2013; Bini \& Hernández Pando, 2014; Daniele, 2014; Taheri et al., 2015; Tyagi et al., 2016; Chaudhary et al., 2017; Vita et al., 2017).

At any age, TBM is one of the most severe extra-pulmonary manifestations of TB, with high short-term mortality and substantial excess morbidity among survivors: approximately one-third of the affected individuals die of the disease and up to one-half of the survivors remain with serious neurological sequels, despite the initiation of antituberculosis therapy. Early, accurate diagnosis and prompt, adequate treatment are crucial in determining the clinical outcome of TBM; nevertheless, these are hampered by several factors: the presenting clinical and radiological features of the disease are often non-specific and may mimic those of other infectious or non-infectious conditions; the conventional laboratory tests may be slow and/or relatively insensitive; and the choice of anti-tuberculosis drugs, dosage regimen, and therapy duration are empiric in TBM and largely based on the experience in treatment of pulmonary tuberculosis. The emergence of MDR-TB further complicates the management of the disease by causing a delay in diagnosis and limiting therapeutic options: drug-susceptibility tests may be time-consuming and some of the second-line drugs have a poor cerebrospinal fluid (CSF) penetration. If left untreated, TBM usually leads to death within 5 to 8 weeks after the onset of its symptoms; although, in some cases (e.g., Griffith, 1919; Schmidt, 1941; Green, 1943; Saito, 1956; Traub et al., 1986; Kent et al., 1993; Newton, 1994), the disease has a protracted course that can last for several months or even years (Thwaites et al., 2002; Bernaerts et al., 2003; Arbeláez et al., 2004; Gauba \& Varma, 2005; Thwaites \& Tran, 2005; Bill, 2006; Be et al., 2009; Garg, 2010; Christensen et al., 2011; Marx \& Chan, 2011; Brancusi et al., 2012; Thwaites, 2013; Bini \& Hernández Pando, 2014; Daniele, 2014; Rajashekar et al., 2014; van Toorn \& Solomons, 2014; Ramirez-Lapausa et al., 2015; Taheri et al., 2015; Vita et al., 2017).

As for its pathogenesis, TBM usually develops subsequent to the rupture of one or more meningeal, subpial, and/or subependymal caseating Rich foci into the subarachnoid 
space or into the ventricular system, both occupied by the CSF. The release of sufficient numbers of $\mathrm{TB}$ bacteria into the CSF triggers the onset of diffuse granulomatous inflammation of the leptomeninges, with a strong predilection for the basal areas of the brain. Nonetheless, not only the pia and arachnoid mater but additionally, the dura mater can be affected by the disease. Besides the small tubercles primarily formed in the leptomeninges and later also in the dura mater, characteristic pathological features of TBM include enhancing basal meningeal exudate, progressive hydrocephalus, and vasculitis of blood vessels adjacent to or traversing the exudate (Aschoff, 1936; Garg, 1999, 2010; Bernaerts et al., 2003; Arbeláez et al., 2004; Katti, 2004; Donald et al., 2005; Gauba \& Varma, 2005; Bill, 2006; Be et al., 2009; Vinnard \& Macgregor, 2009; Christensen et al., 2011; Bano et al., 2012; Brancusi et al., 2012; Bini \& Hernández Pando, 2014; Rajashekar et al., 2014; Taheri et al., 2015; Chaudhary et al., 2017).

In the initial stage of TBM, the granulomatous inflammatory reaction results in the formation of a thick, gelatinous or slightly nodular exudate between the two layers of the leptomeninges, primarily located in close vicinity to the basal subarachnoid cisterns, along the infero-medial surface of the frontal lobes, the antero-medial surface of the temporal lobes, the floor of the third ventricle, and the superior aspect of the cerebellum. From here, the inflammatory exudate rapidly extends towards the basal subarachnoid cisterns (i.e., the interpeduncular and suprasellar cisterns). As the disease progresses, the infection may gradually spread to other subarachnoid cisterns, such as the prepontine, ambient, and Sylvian cisterns, and eventually, it can reach the meninges covering the cerebral convexities, the ependymal surface of the ventricles, and the choroid plexuses (Bernaerts et al., 2003; Arbeláez et al., 2004; Gauba \& Varma, 2005; Rock et al., 2008; Vinnard \& Macgregor, 2009; Bano et al., 2012; Raut et al., 2013; Taheri et al., 2015; Chaudhary et al., 2017).

The inflammatory exudate, partially or completely filling the subarachnoid space and the ventricular pathways, may result in the development of persistent and progressive hydrocephalus (i.e., disturbance of CSF flow, reabsorption or production, leading to an increase in volume occupied by the cerebrospinal fluid in the CNS) that is one of the most common complications of tuberculous meningitis, occurring in more than two-thirds of the cases. In TBM, either the communicating or the non-communicating form of hydrocephalus can develop, with the former type being more frequent. The communicating type of TB hydrocephalus generally occurs when the inflammatory exudate blocks the flow of the cerebrospinal fluid within the subarachnoid space, resulting in impaired reabsorption of the CSF. In later stages of the disease, inflammation of the ependymal surface of the ventricles 
and of the choroid plexuses leads to overproduction of the CSF, also contributing to the progression of communicating TB hydrocephalus. The non-communicating form of TB hydrocephalus develops when the inflammatory exudate obstructs the pathways connecting the ventricles (i.e., the foramina of Monro and the aqueduct of Sylvius) or the passages between the fourth ventricle and the subarachnoid space (i.e., the foramina of Luschka and the foramen of Magendie), resulting in blockage of the CSF flow. Tuberculous hydrocephalus is often associated with elevated intracranial pressure (eICP) (Bernaerts et al., 2003; Arbeláez et al., 2004; Gauba \& Varma, 2005; Rajshekhar, 2009; Vinnard \& Macgregor, 2009; Bano et al., 2012; Morgado et al., 2013; Nielsen \& Breedt, 2013; Raut et al., 2013; van Toorn \& Solomons, 2014; Taheri et al., 2015; Tyagi et al., 2016).

Since the late $20^{\text {th }}$ century, palaeopathological studies on osteoarchaeological series (e.g., Schultz, 1993, 1999, 2001, 2003; Templin \& Schultz, 1994; Teschler-Nicola et al., 1994, 2015; Jankauskas \& Schultz, 1995; Jankauskas, 1999; Maczel, 2003; Schultz \& Schmidt-Schultz, 2015) and documented skeletal collections (e.g., Hershkovitz et al., 2002; Maczel, 2003; Schultz \& Schmidt-Schultz, 2015) have revealed a positive correlation between TBM and a number of endocranial bony alterations, namely abnormally pronounced digital impressions (APDIs), periosteal appositions (PAs), abnormal blood vessel impressions (ABVIs), serpens endorcrania symmetrica (SES), and granular impressions (GIs).

Digital impressions (DIs), also known as convolutional markings, on the inner surface of the skull (i.e., shallow depressions resembling to the imprint of a finger and corresponding to cerebral gyri that are intervened by thicker bony ridges corresponding to cerebral sulci) are probably formed by localised pressure of the pulsating brain underlying the bone. Although very pronounced DIs, generally confined to the base and lower two-thirds of the skull vault, may be normal in childhood (particularly during periods of rapid brain growth), their prominence decreases during adolescence. In adulthood, the presence of abnormally pronounced digital impressions (Fig. 8) over the upper portion of the skull vault indicates a prolonged rise in the intracranial pressure: according to estimates, the formation of APDIs secondary to eICP requires at least 10 weeks. Although APDIs can be caused by TB hydrocephalus accompanied by eICP, they are not pathognomonic features of TBM, since other pathological conditions (such as other CNS infections, brain tumours or haemorrhages) can also result in the development of eICP (Schüller, 1940-41; du Boulay, 1956, 1980; Bell, 1978; Schultz, 1993, 2001, 2003; Mahomed et al., 2012; Paul et al., 2013, 2014; Desai et al., 2014; Pemmaiah, 2015). 




Figure 8: APDIs on the endocranial surface of the squamous part of the frontal bone (Eperjes - Ifjú Gárda Tsz., Hungary, $10^{\text {th }}-11^{\text {th }}$ century AD, Grave No. 4/A, Infantia II) (Spekker et al., 2014).

Abnormal blood vessel impressions (Fig. 9A-B, 10A-B) and periosteal appositions (Fig. 9B, 10A-B) localised on the inner surface of the skull were recognised as vestiges of inflammatory and/or haemorrhagic processes of the meninges (e.g., Mensforth $e t$ al., 1978; Schultz, 1993, 1999, 2001, 2003; Maczel, 2003; Lewis, 2004). These non-specific bony changes may be generated by a number of infectious (e.g., non-specific and specific meningitis) and non-infectious (e.g., trauma and scurvy) conditions, including TBM. The initial stage of the haemorrhagic process involves the formation of small, patch-like areas of very short, sinuous, branching blood vessel impressions extending into the endocranial lamina of the skull consequent to the development of an epidural haemorrhage. As the meningeal process progresses, ABVIs may be covered by appositions of newly formed bone (PAs) with a fibrous, porous, irregular, scab-like appearance. In more advanced stages, the groups of tongue-like PAs with a very smooth, more mature appearance are separated by an extensive net-like aggregation of ABVIs. Bony changes resulted from haemorrhagic meningeal reactions can only be found external to the original bone surface. The inflammatory meningeal process is expressed by small, flat, plate-like appositions of newly built bone (PAs) that are oriented tangentially to the endocranial surface of the affected bone. Later, isolated plates may be confluent as one or more layers, their contours become indistinct, and their plate-like character will be lost as a consequence of bone remodelling. 
In contrast to the haemorrhagic meningeal processes, the inflammatory reactions always affect the original bone surface, frequently also the deeper structures of the original bone material. Both ABVIs and PAs are generally situated in the APDIs; nevertheless, they may spread over larger areas of the skull vault in more advanced stages. Although the bony vestiges of meningeal processes may be confused by macroscopic examinations only, they can be differentiated by microscopic investigations (e.g., polarised light microscopy) into inflammatory, haemorrhagic, and mixed forms (Mensforth et al., 1978; Schultz, 1993, 2001, 2003).

A)



B)



Figure 9: A) ABVIs on the endocranial surface of the squamous part of the frontal bone (Vésztö Mágori-halom, Hungary, $4^{\text {th }}-49^{\text {th }}$ century BC, Grave No. 5, Infantia II) (Spekker et al., 2012) and B) small ABVIs accompanied by PAs on the inner surface of the frontal bone

(Tápé - Széntéglaégetô, Hungary, $12^{\text {th }}-13^{\text {th }}$ century BC, Grave No. 61, Infantia I) (Spekker et al., 2016). 
Figure 10: Newly built bone formations (PAs) and abnormal blood vessel impressions (white arrows) representing haemorrhagic-inflammatory processes of the meninges probably due to tuberculosis on the endocranial surface of the frontal bone of an adult individual (historic collection of the Department of Pathology, University of Göttingen, Göttingen, Germany; GP-1985616). Thin-ground sections (A): thickness: $50 \mu \mathrm{m}$, magnification: $25 x$ and B) thickness: $70 \mu \mathrm{m}$, magnification: $25 \times 1.6 x)$ viewed in polarised light using a hilfsobject red $1^{\text {st }}$ order (quartz) as compensator (photos by Michael Schultz) (Schultz, 2003).

A)



B)




During the examination of skeletons of known cause of death from the Hamann-Todd Human Osteological Collection, Hershkovitz and his colleagues (2002) observed serpentine branching surface excavations characterised by a maze-like appearance (Fig. 11) predominantly in specimens identified to have died of respiratory diseases, particularly of tuberculosis. They termed these lesions, generally located on the endocranial surface of the frontal bone (predominantly around its most protruding parts), parietal bones (particularly around their most protruding part or along the superior sagittal sinus), and occipital bone (mainly along the dominant transverse sinus), as "serpens endocrania symmetrica". SES may represent an advanced stage of the haemorrhagic process of the meninges: Hershkovitz and his co-workers (2002) attributed it to vascular anomaly, namely to changes in the primary and secondary anastomotic arteries traversing the dura mater, subsequent to the development of an epidural haemorrhage. The pathological process leading to the formation of SES affects only the superficial part of the endocranial lamina of the skull with no diploic and/or ectocranial involvement (Hershkovitz et al., 2002; Maczel, 2003; Lewis, 2004).



Figure 11: SES along the sagittal and transverse sinuses on the endocranial surface of the squamous part of the occipital bone (Tápé - Széntéglaégető, Hungary, $12^{\text {th }}-13^{\text {th }}$ century BC,

Grave No. 608, Juvenis, male) (Spekker et al., 2016). 
In contrast to the above-mentioned endocranial alterations, $\operatorname{Schultz}(1999,2001,2003)$ described small (0.5-1.0 $\mathrm{mm}$ in diameter), relatively shallow (less than $0.5 \mathrm{~mm}$ in depth), roundish impressions with smooth margins and walls (Fig. 12, 13A-B), generally appearing as isolated or confluent lesions grouped in clusters on the inner surface of the skull base or sometimes of the lateral skull vault, as pathognomonic features of TBM. These granular impressions - particularly situated in the greater wings of the sphenoid bone, the squamous part of the temporal bones, and the lateral and squamous parts of the occipital bone - may be established by pressure atrophy of the tubercles formed in the dura mater during later stages of TBM. Although GIs were named as sharply demarcated erosive defects by Hershkovitz and his colleagues (2002), the lesions have an erosive appearance with more irregular shape and sharper walls and margins (Fig. 13A, C) only when the pathological process progresses, and the tubercles resulting in the impressions become caseating. Similar to the aforementioned endocranial alteration types, GIs represent a pathological process that affects only the superficial part of the inner lamina of the skull with no diploic and/or ectocranial involvement (Schultz, 1999, 2001, 2003; Hershkovitz et al., 2002; Maczel, 2003; Schultz \& Schmidt-Schultz, 2015).



Figure 12: GIs accompanied by ABVIs on the endocranial surface of the right parietal bone (Csongrád - Felgyő (Ürmös-tanya), Hungary, $7^{\text {th }}-8^{\text {th }}$ century AD, Grave No. 221, Juvenis, female?)

(Maczel, 2003). 
Figure 13: Granular impressions probably caused by pressure atrophy of the tubercles on the endocranial surface of the frontal bone (right side) of a 22-year-old male (individual 59) from the Weisbach Collection (Vienna, Austria; end of the $19^{\text {th }}$ century) who was clinically diagnosed with TB: A) group of three GIs (arrows) (thickness: $70 \mu \mathrm{m}$; magnification: 16x), B) a flat, roundish impression with smooth walls (thickness: $70 \mu \mathrm{m}$; magnification: 100x), and C) an erosive impression with sharper walls (thickness: $70 \mu \mathrm{m}$; magnification: 100x). Thin-ground sections viewed in polarised light using a hilfsobject red $1^{\text {st }}$ order (quartz) as compensator (photos by Michael Schultz) (Schultz, 2001, 2003).

A)



B)



C)




2 AIMS \& OBJECTIVES 
Using modern medical knowledge, palaeopathologists attempt to establish a retrospective diagnosis of prehistoric and historic TB cases by identifying pathological conditions in bone remains of people lived in the past that may be related to tuberculosis (e.g., vertebral changes, rib lesions, and endocranial alterations). However, on the one hand, probable TB-associated bony changes observed in recent cases may differ from those of detectable in skeletons from archaeological sites, due in part to the introduction of antibiotics in the treatment of tuberculosis. On the other hand, in modern clinical cases, bony lesions cannot be surveyed with macromorphological methods but with medical imaging techniques (e.g., X-ray radiography, computed tomography, and magnetic resonance imaging) only; nevertheless, subtle bony alterations probably related to TB (e.g., PNBFs) may be impossible to be visualised by the latter ones. Therefore, they are not relevant to the diagnosis of tuberculosis in recent cases and are not described as diagnostic criteria for the disease by physicians in the modern medical literature, even if they could be potentially important elements of TB identification for palaeopathologists. Thus, utilisation of modern diagnostic criteria for tuberculosis in the palaeopathological practice may not be appropriate (Santos \& Roberts, 2001, 2006; Ortner, 2003; Roberts \& Buikstra, 2003; Matos \& Santos, 2006).

Nonetheless, detailed analysis of well-documented collections of pre-antibiotic era skeletons of known cause of death can serve as a unique and important basis for the diagnosis of TB in past human populations, since bone remains of specimens identified to have died of tuberculosis and not treated with antibiotics may exhibit similar TB-related bony changes to those of observable in skeletons from archaeological sites; they can be directly surveyed with macromorphological methods; and even subtle bony changes can be recognised in them. Therefore, examination of such collections (e.g., Hamann-Todd Human Osteological Collection, Robert J. Terry Anatomical Skeletal Collection, and Coimbra Identified Skeletal Collection) can contribute to determining the appropriate palaeopathological diagnostic criteria for TB (Santos \& Roberts, 2001, 2006; Matos \& Santos, 2006; Pálfi et al., 2012; Mariotti et al., 2015).

In the last three decades, the Terry Collection has been used to define and refine palaeopathological diagnostic criteria for tuberculosis in several studies (e.g., Roberts et al., 1994; Winland et al., 1997; Rothschild \& Rothschild, 1998, 1999; Pálfi et al., 2012); however, probable TB-associated endocranial alterations were beyond the scope of the aforementioned research projects. Although a number of endocranial bony alterations (e.g., APDIs; PAs; ABVIs, including SES; and GIs) have been attributed to TB infection in the palaeopathological literature, their diagnostic value has more recently been questioned (e.g., 
Lewis, 2004; Roberts et al., 2009; Janovic et al., 2015), as their precise aetiology is still a matter of controversy, and additionally, similar or even the same morphological features can be found in non-TB-related cases, such as in non-specific inflammatory (e.g., bacterial meningitis) and haemorrhagic (e.g., epidural haematoma) processes.

The main aim of the present PhD dissertation is to expand knowledge and understanding about the development of probable TB-associated endocranial alterations and to improve their palaeopathological interpretation, as well as to contribute to strengthening their diagnostic value in the identification of TB in human osteoarchaeological material. Therefore, review of the modern medical and palaeopathological literature regarding tuberculosis was conducted, with special attention to bony changes likely related to TB. Furthermore, a detailed investigation focusing on the macromorphological characteristics and frequencies of the above-mentioned lesions, as well as of their co-occurrence with each other and with non-endocranial bony changes probably related to $\mathrm{TB}$, was performed on all individuals recorded to have died of different types of tuberculosis in the Terry Collection, and on a control group consisting of randomly selected specimens from the remaining skeletons of the Terry Collection, identified to have died of causes other than TB.

\section{The objectives of the present $\mathrm{PhD}$ dissertation are the following:}

1) To macroscopically evaluate skeletons from the Terry Collection for the presence of the four types of probable TBM-associated endocranial alterations, as well as for their cooccurrence with each other and with non-endocranial lesions possibly related to TB;

2) To compare the frequencies of the four types of probable TBM-related endocranial alterations, as well as of their co-occurrence with each other and with non-endocranial bony changes possibly associated with tuberculosis, between individuals recorded to have died of $\mathrm{TB}$ and specimens identified to have died of causes other than $\mathrm{TB}$, considering sex and age at death of individuals;

3) To macromorphologically characterise the four probable TBM-associated endocranial alteration types detected in skeletons from the Terry Collection regarding the prominence (APDIs), as well as the localisation, extent, and number (PAs, ABVIs, and GIs) of lesions on the affected cranial bone(s);

4) To provide example cases showing the most important macromorphological characteristics of the four types of possible TBM-related endocranial alterations; and

5) To evaluate the diagnostic value of the four probable TBM-associated endocranial alteration types examined in skeletons from the Terry Collection. 
3 MATERIALS \& METHODS 


\subsection{The Robert J. Terry Anatomical Skeletal Collection}

The Robert J. Terry Anatomical Skeletal Collection - currently curated in the Department of Anthropology at the National Museum of Natural History (NMNH) (Smithsonian Institution, Washington, DC, USA) (Fig. 14A-B) - consists of 1,728 human skeletons (1,011 males and 717 females) that were accumulated first by Robert J. Terry, professor of anatomy and head of the Anatomy Department at Washington University Medical School in St. Louis (Missouri, USA), from the second decade of the $20^{\text {th }}$ century until his retirement in 1941, and later by Mildred Trotter, who succeeded Terry as anatomy professor, between 1941 and 1967. In the Terry Collection, individuals were born between 1828 and 1943 and died between 1905 and 1966, with age at death ranging from 16 to 102 years. Owing to Terry's well-established uniform protocol for the collecting, cataloguing, maceration, and storage of bone remains, almost all of the skeletons in the Terry Collection are complete and well-preserved, and for each of them, a series of documentary forms (e.g., morgue record, dental chart, anthropometric and anthroposcopic data form, and bone inventory list) providing various biographical information (e.g., name, sex, age at death, "race", occupation, and cause of death) and basic anthropological data is available at the NMNH. Therefore, the Terry Collection serves as an invaluable resource for anthropological and medical research, including developing new criteria for the diagnosis of specific infectious diseases (such as tuberculosis) in osteoarchaeological material from the preantibiotic era (Hunt \& Albanese, 2005).

A)



B)



Figure 14: Current locations of skeletons from the Terry Collection: A) Natural History Building and B) Museum Support Center (http://2.bp.blogspo t.com/-gMOj8xL8pC8/T9-rS_HBYNI/AAAAAAAAAOU/1P-DVfCXIko/s1600/IMG_1616.jpg). 


\subsection{Materials and methods}

As part of a comprehensive research project, a detailed investigation (Fig. 15A-C) focusing on the macromorphological characteristics, frequencies, and co-occurrences of different types of pathological bony alterations probably related to tuberculosis (e.g., vertebral changes, rib lesions, and endocranial alterations) was performed on all specimens $(\mathrm{N}=302)$ recorded to have died of different types of TB (e.g., pulmonary TB, miliary TB, peritoneal TB, and skeletal TB) in the Terry Collection (Suppl. table 1), and on a control group (NTB group) consisting of randomly selected individuals $(\mathrm{N}=302)$ from the remaining specimens of the Terry Collection, identified to have died of causes other than TB (e.g., other infectious diseases, cardiovascular problems, cancer, and external causes, such as suicide or homicide) (Suppl. table 2). It must be noted that even if the recorded cause of death may not have been tuberculosis, individuals could still have suffered from the disease but their death was attributed to another cause (Roberts et al., 1994; Santos \& Roberts, 2001).

A)



B)



C)



Figure 15: A) Selection, B) moving, and C) evaluation of skeletons in the Terry Collection.

From the 604 skeletons surveyed in the Terry Collection, 177 were excluded from the examination considering endocranial alterations probably associated with TBM: the skullcap was missing in two cases and the skull was not sectioned in a further 173 cases; therefore, precluding the accurate observation of the inner surface of the skull; whereas the age at death was uncertain in two additional cases; thus, compromising the statistical analysis of data. The remaining 427 late adolescent (16-19 years old; $\Sigma=7$; three males and four females) and adult ( $>20$ years old; $\Sigma=420 ; 272$ males and 148 females) individuals with skulls sectioned 
in the transverse plane and occasionally also in the mid-sagittal plane were divided into two main groups on the basis of their causes of death:

1) TB group, consisting of 234 specimens (169 males and 65 females) identified to have died of TB, with age at death ranging from 16 to 81 years (Fig. 16, Suppl. table 1); and



$\square$ Males $(\Sigma=169)$

$\square$ Females $(\Sigma=65)$

Figure 16: Demographic profile of specimens surveyed in the TB group $(\Sigma=234)$.

2) Control (NTB) group, composed of 193 individuals (106 males and 87 females) recorded to have died of causes other than $\mathrm{TB}$, with age at death ranging from 20 to 90 years (Fig. 17, Suppl. table 2).



Figure 17: Demographic profile of specimens surveyed in the NTB group $(\Sigma=193)$.

The endocranial surface of the selected skulls was macroscopically surveyed for the presence of the four types of endocranial alterations probably related to TBM (i.e., periosteal appositions (Fig. 18A); abnormal blood vessel impressions, including SES (Fig. 18B); granular impressions (Fig. 18C); and abnormally pronounced digital impressions (Fig. 18D)), without prior knowledge of the cause of death of specimens. 
A)



C)



B)



D)



Figure 18: Different types of endocranial alterations probably related to TBM:

A) PAs in the left parietal bone (Terry No. 987, 23-year-old, male, pulmonary TB), B) ABVIs on the right side of the squamous part of the frontal bone (Terry No. 329, 18-year-old, male, pulmonary TB),

C) GIs on the left side of the orbital part of the frontal bone (Terry No. 1159, 26-year-old, male, pulmonary TB), and D) APDIs in the right parietal bone (Terry No. 89R, c. 32-year-old, male, TB).

During the macromorphological investigation, a lamp was always positioned at a distance of few centimetres from the bone surface, since the examined bony changes can have a very subtle appearance, making their detection difficult. For each selected individual, detailed written and pictorial descriptions of all observed endocranial alterations probably associated with TBM were made on a data collection sheet prepared for the current research project (Table 1). 


\begin{tabular}{|c|c|c|c|c|c|c|c|c|}
\hline \multirow{2}{*}{$\begin{array}{c}\text { Terry No. } \\
1159\end{array}$} & \multirow{2}{*}{$\begin{array}{c}\text { Frontal } \\
\text { bone }\end{array}$} & \multicolumn{2}{|c|}{ Parietal bones } & \multicolumn{2}{|c|}{ Temporal bones } & \multicolumn{2}{|c|}{ Sphenoid bone } & \multirow{2}{*}{$\begin{array}{c}\text { Occipital } \\
\text { bone }\end{array}$} \\
\hline & & Left & Right & Left & Right & Left & Right & \\
\hline PAs & M 1 & M 1 & M 1 & M 1 & M 1 & $\mathbf{0}$ & $\mathbf{0}$ & M 1 \\
\hline ABVIs & $\mathbf{0}$ & $\mathbf{0}$ & $\mathbf{0}$ & $\mathbf{0}$ & $\mathbf{0}$ & $\mathbf{0}$ & $\mathbf{0}$ & $\mathbf{0}$ \\
\hline GIs & M 1 & $\mathbf{0}$ & $\mathbf{0}$ & $\mathbf{0}$ & $\mathbf{0}$ & $\mathbf{0}$ & $\mathbf{0}$ & M 1 \\
\hline \multicolumn{8}{|c|}{ Abnormally pronounced digital impressions (APDIs) } & + \\
\hline
\end{tabular}

Table 1: Data collection sheet for recording endocranial alteration types probably related to TBM. Example case: Terry No. 1159, 26-year-old, male, pulmonary TB $(0$ not present, $M=$ multifocal, $1=$ stage $1(x<25 \%),+$ = very slight $)$.

With respect to the PAs, ABVIs (Fig. 19A-C), and GIs (Fig. 20A-C), the affected cranial bone(s) (considering the left and right greater wings of the sphenoid bone as two separate bones); the number of detected lesions in the affected cranial bone(s) (unifocal or multifocal); and the extent of the endocranial surface area the observed lesion(s) covered (x) in the affected cranial bone(s) (4-level scale: 1) $\mathrm{x}<25 \%$, 2) $25 \% \leq \mathrm{x}<50 \%$, 3) $50 \% \leq \mathrm{x}<$ $75 \%$, and 4) $75 \% \leq x$ ) were recorded; whereas concerning the APDIs (Fig. 21A-C), their prominence was registered (3-level scale: 1) very slight: shallow DIs $(<2 \mathrm{~mm}$ in depth) predominantly over the anterior portion of the endocranial surface, 2) slight: deeper DIs (2$4 \mathrm{~mm}$ in depth) particularly over the anterior and middle portions of the endocranial surface, and 3) pronounced: deep DIs (4 $\mathrm{mm}<$ in depth) all over the endocranial surface) (Table 1). Besides the above-mentioned endocranial alterations, the presence of different types of nonendocranial bony lesions probably related to TB (e.g., vertebral changes, rib lesions, and joint alterations) was also recorded in the 427 selected specimens from the Terry Collection.

After the detailed macromorphological evaluation of the skeletons, all information collected was entered into a spreadsheet in Microsoft Office Excel 2010, and subsequent statistical analysis of data was performed: absolute and percentage frequencies of the four types of endocranial alterations probably associated with TBM, as well as of their cooccurrence with each other and with non-endocranial bony changes possibly related to TB, were calculated in both the TB group and NTB group; and $\chi^{2}$ testing of data to determine the significance of differences (if any) in frequencies of all examined endocranial alteration types, as well as of their association with each other and with non-endocranial bony lesions probably related to TB between the two groups, was undertaken using the MedCalc statistical software package. 
Figure 19: Different stages of multifocal PAs and ABVIs on the squamous part of the frontal bone: A) stage 2 (Terry No. 1322, 34-year-old, male, pulmonary TB), B) stage 3 (Terry No. 306, 18-year-old, female, pulmonary TB), and C) stage 4 (Terry No. 254, 21-year-old, male, pulmonary TB).

A)



B)



C)




Figure 20: Different stages of multifocal GIs on the squamous part of the occipital bone:

A) stage 1 (Terry No. 1106, 28-year-old, male, skeletal TB), B) stage 2 (Terry No. 933R, 40-year-old, male, peritoneal TB), and C) stage 3 (Terry No. 562, 17-year-old, female, pulmonary TB).

A)



B)



C)




Figure 21: Different stages of the prominence of APDIs on the squamous part of the frontal bone: A) very slight (Terry No. 1036, 38-year-old, male, pulmonary TB), B) slight (Terry No. 265, 32-year-old, male, TB), and C) pronounced (Terry No. 251, 34-year-old, male, pulmonary TB).

A)



B)



C)




4 RESULTS \& CASE STUDIES 


\subsection{Endocranial alteration types probably related to tuberculosis}

\subsubsection{Abnormally pronounced digital impressions}

\begin{tabular}{|c|c|c|c|c|c|}
\hline \multicolumn{2}{|c|}{$\Sigma=427$} & \multicolumn{2}{|c|}{ TB group } & \multicolumn{2}{|c|}{ NTB group } \\
\hline \multicolumn{2}{|c|}{$\begin{array}{l}\text { Number of specimens } \\
\text { affected by APDIs }\end{array}$} & \multicolumn{2}{|c|}{$\begin{array}{c}154 / 234 \\
(65.81 \%)\end{array}$} & \multicolumn{2}{|c|}{$\begin{array}{c}62 / 193 \\
(32.12 \%)\end{array}$} \\
\hline Females & Males & $\begin{array}{c}34 / 65 \\
(52.31 \%)\end{array}$ & $\begin{array}{c}120 / 169 \\
(71.01 \%)\end{array}$ & $\begin{array}{c}19 / 87 \\
(21.84 \%)\end{array}$ & $\begin{array}{c}43 / 106 \\
(40.57 \%)\end{array}$ \\
\hline
\end{tabular}

Table 2: Number of specimens exhibiting APDIs in the TB group and NTB group by sex.

From a total of 427 skeletons evaluated, 216 (50.59\%) exhibited APDIs on the inner surface of the skull: 154 (65.81\%) of 234 individuals recorded to have died of TB and 62 (32.12\%) of 193 specimens identified to have died of causes other than TB (Table 2); thus, there was a statistically extremely significant difference in the frequencies of APDIs between the TB group and NTB group $\left(\chi^{2}=46.680, \mathrm{df}=1, \mathrm{P}<0.0001\right)$. When the two groups were compared considering the sex (Table 2), the difference in the frequencies of APDIs remained significant for both females $\left(\chi^{2}=13.896, \mathrm{df}=1, \mathrm{P}=0.0002\right)$ and males $\left(\chi^{2}=23.759\right.$, $\mathrm{df}=1, \mathrm{P}<0.0001)$. Furthermore, an approximately 20 percentage point difference in the frequencies of APDIs between females and males was found in both groups: $52.31 \%$ versus $71.01 \%$ in the TB group and $21.84 \%$ versus $40.57 \%$ in the NTB group, respectively (Table 2). The $\chi^{2}$ comparison of the frequencies of APDIs between the two sexes (Table 2) revealed a statistically significant difference in both individuals with $\mathrm{TB}$ as the cause of death $\left(\chi^{2}=6.487, \mathrm{df}=1, \mathrm{P}=0.0109\right)$ and specimens with NTB causes of death $\left(\chi^{2}=6.850, \mathrm{df}=1\right.$, $\mathrm{P}=0.0089$ ).

Concerning the distribution of affected individuals by age at death, APDIs occurred with the highest frequency among specimens under the age of 30 years $(61 / 70,87.14 \%)$ : with $88.89 \%$ (56/63) in the TB group (Fig. 22A) and with $71.43 \%(5 / 7)$ in the NTB group (Fig. 23A). Of females and males under the age of 30 years and identified to have died of TB, 81.82\% (18/22) (Fig. 22B) and 92.68\% (38/41) (Fig. 22C) showed APDIs, respectively. Nevertheless, more than one-half of females between 30 and 49 years of age $(14 / 25,56.00 \%)$ (Fig. 22B) and more than two-thirds of males between 30 and 59 years of age (70/104, 67.31\%) (Fig. 22C) also exhibited APDIs in the TB group. Moreover, among individuals recorded to have died of causes other than TB, besides females $(2 / 3,66.67 \%)$ (Fig. 23B) and males $(3 / 4,75.00 \%)$ (Fig. 23C) under the age of 30 years, males between 60 and 69 years of age $(12 / 22,54.55 \%)$ (Fig. 23C) were most frequently affected by APDIs. The $\chi^{2}$ testing of the frequencies of APDIs between various age groups was not assessed because of the low number of specimens in certain age groups. 
Figure 22: Demographic profile of specimens exhibiting APDIs in the TB group:

A) total sample $(154 / 234,65.81 \%)$, B) females $(34 / 65,52.31 \%)$, and C) males $(120 / 169,71.01 \%)$.

A)



$\square$ Number of specimens $(\Sigma=234) \quad \square$ Number of specimens affected by APDIs $(\Sigma=154)$

B)



$\square$ Number of females $(\Sigma=65) \quad \square$ Number of females affected by APDIs $(\Sigma=34)$

C)



$\square$ Number of males $(\Sigma=169) \quad \square$ Number of males affected by APDIs $(\Sigma=120)$ 
Figure 23: Demographic profile of specimens exhibiting APDIs in the NTB group:

A) total sample $(62 / 193,32.12 \%)$, B) females (19/87, 21.84\%), and C) males (43/106, 40.57\%).

A)



$\square$ Number of specimens $(\Sigma=193) \quad \square$ Number of specimens affected by APDIs $(\Sigma=62)$

B)



$\square$ Number of females $(\Sigma=87) \quad \square$ Number of females affected by APDIs $(\Sigma=19)$

C)



$\square$ Number of males $(\Sigma=106) \quad \square$ Number of males affected by APDIs $(\Sigma=43)$ 
As for the prominence of APDIs, from the 216 skulls affected, 148 (68.52\%), 51 (23.61\%), and 17 (7.87\%) had very slight (Fig. 24A, 25A-B), slight (Fig. 24B, 25A-B), and pronounced APDIs (Fig. 24C-D, 25A-B), respectively.

A)



C)



B)



D)



Figure 24: Different stages of the prominence of APDIs on the inner surface of the skull: A) very slight (skullcap, Terry No. 30R, 26-year-old, male, TB meningitis), B) slight (skullcap, Terry No. 382R, 26year-old, male, pulmonary TB), C) pronounced (skullcap, Terry No. 1033, 26-year-old, male, pulmonary TB), and D) pronounced (skull base, Terry No. 1033, 26-year-old, male, pulmonary TB). 
Although the very slight stage of the prominence of APDIs was more common among specimens with NTB causes of death (Fig. 24A, 25A-B) and the more pronounced (i.e., slight and pronounced) stages of the prominence of APDIs occurred more frequently among individuals with TB as the cause of death (Fig. 24B-D, 25A-B), statistically significant difference between the two groups was found only in the frequencies of the very slight stage of the prominence of APDIs (very slight: $\chi^{2}=8.530, \mathrm{df}=1, \mathrm{P}=0.0035$; slight: $\chi^{2}=3.312, \mathrm{df}=1$, $\mathrm{P}=0.0688$; and pronounced: $\chi^{2}=3.564, \mathrm{df}=1, \mathrm{P}=0.0591$ ).

A)



B)



Figure 25: Distribution of specimens affected by APDIs in the A) TB group ( $\Sigma=154)$ and B) NTB group ( $\Sigma=62)$ by stages of the prominence of APDIs.

Of the 154 specimens with APDIs in the TB group, 124 were identified to have died of pulmonary TB (Suppl. table 1, 3). Nine additional individuals died of other types of tuberculosis, such as skeletal TB (four cases), peritoneal TB (two cases), TB meningitis (two cases), and miliary TB (one case); whereas in the remaining 21 cases, the type of tuberculosis as the cause of death was not specified on the morgue record and/or death certificate (Suppl. table 1, 3). In the NTB group, the most frequently registered NTB causes of death were cardiovascular problems, followed by respiratory diseases, infectious diseases other than $\mathrm{TB}$, and different types of cancer among specimens exhibiting APDIs on the inner surface of the skull (Suppl. table 2, 4).

In the skeleton of Terry No. 30R - a 26-year-old male recorded to have died of TBM (Suppl. table 1) -, pathological bony changes that may be attributed to tuberculosis were registered both in the cranial and postcranial elements. Regarding the skull, shallow APDIs (very slight stage) were noted on the squamous part of the frontal bone (Fig. 24A, 26), probably referring to eICP secondary to TBM (e.g., Schultz, 1993, 2001, 2003). Furthermore, GIs - described by Schultz (e.g., 1999, 2001, 2003) and Schultz \& Schmidt- 
Schultz (2015) as pathognomonic vestiges of TBM, since representing pressure atrophy of the tubercles that affect the dura mater - were detected on the orbital part of the frontal bone and on the squamous part of the occipital bone, covering less than one-fourth (stage 1) of the inner surfaces.



Figure 26: Shallow APDIs (very slight stage) on both sides of the squamous part of the frontal bone (Terry No. 30R, 26-year-old, male, TB meningitis).

Besides the endocranial alterations very likely associated with TBM, in the vertebral column, signs of hypervascularisation were noted in the form of circumferential pitting on the lateral and anterior aspects of the lower thoracic (T10-12), as well as on the lateral aspects of the lumbar (L1-5) vertebral bodies. Although vertebral hypervascularisation is not a specific feature of tuberculosis, it has been described in relation to early-stage skeletal TB in a number of studies (e.g., Ménard, 1888; Baker, 1999; Maczel, 2003; Giacon, 2008; Mariotti et al., 2015). The recorded cause of death of Terry No. 30R supports the tuberculous origin of the observed endocranial and vertebral changes.

The skeletal remains of Terry No. 382R - a 26-year-old male whose morgue record states pulmonary TB as the cause of death (Suppl. table 1) - exhibited numerous pathological bony changes that probably resulted from tuberculosis. In the skull, APDIs (slight stage) affecting the squamous part of the frontal bone (Fig. 24B, 27) and the left and right parietal bones (Fig. 24B) were registered, indicating eICP possibly due to hydrocephalus that may be associated with TBM (e.g., Schultz, 1993, 2001, 2003). 




Figure 27: APDIs (slight stage) on both sides of the squamous part of the frontal bone (Terry No. 382R, 26-year-old, male, pulmonary TB).

As for the postcranial skeleton, all left side ribs showed slight PNBFs on the visceral surface of the vertebral end $\left(2^{\text {nd }}-12^{\text {th }}\right)$, body $\left(10^{\text {th }}-12^{\text {th }}\right)$, and/or sternal end $\left(1^{\text {st }}, 5^{\text {th }}-8^{\text {th }}\right.$, and $\left.10^{\text {th }}\right)$. Moreover, slight PNBFs occurred on the vertebral end $\left(2^{\text {nd }}-11^{\text {th }}\right)$ and occasionally on the sternal end $\left(3^{\text {rd }}-7^{\text {th }}\right)$ of ten right side ribs $\left(2^{\text {nd }}-11^{\text {th }}\right)$, exclusively affecting the visceral surfaces. Besides the ribs, the visceral surface of the middle and lower parts of the manubrium and the upper and middle parts of the body of the sternum, as well as the upper part of the costal surface of both scapulae, also revealed slight PNBFs. PNBFs affecting bones of the thoracic region (ribs, sternum, and scapulae) may represent vestiges of an inflammatory response secondary to pulmonary TB and/or TB pleurisy (e.g., Kelley \& Micozzi, 1984; Roberts et al., 1994; Santos \& Roberts, 2001, 2006; Maczel, 2003; Matos \& Santos, 2006; Giacon, 2008; Mariotti et al., 2015). In the vertebral column, signs of hypervascularisation possibly referring to early-stage skeletal TB (e.g., Ménard, 1888; Baker, 1999; Maczel, 2003; Giacon, 2008; Mariotti et al., 2015) were detected on the lateral and anterior aspects of the cervical (C2-7), thoracic (T1-12), and lumbar (L1-5) vertebral bodies, as well as on the ventral surface of the lower part of the sacrum (S3-5). Based on the recorded cause of death of Terry No. 382R, the most likely aetiology of the observed endocranial and postcranial bony changes is tuberculosis.

Similar to the previous cases, both the cranial and postcranial remains of Terry No. 1033 - a 26-year-old male who had died of pulmonary TB (Suppl. table 1) - showed different types of pathological bony changes that may be ascribed to tuberculosis. 
Regarding the cranium, deep APDIs (pronounced stage) were registered all over the inner surface of the skullcap (Fig. 24C, 28) and skull base (Fig. 24D), indicating eICP possibly due to tuberculous involvement of the CNS (e.g., Schultz, 1993, 2001, 2003). Furthermore, non-specific vestiges of haemorrhagic and/or inflammatory meningeal reactions - namely multifocal, small, serpentine branching ABVIs accompanied by slight PAs (e.g., Schultz, 1993, 1999, 2001, 2003) - were noted in the deep DIs located in the frontal (Fig. 28) and the left and right parietal bones, covering less than one-fourth (stage 1) of the inner surfaces.



Figure 28: Multifocal PAs and ABVIs localised in the deep APDIs (pronounced stage) on both sides of the squamous part of the frontal bone (Terry No. 1033, 26-year-old, male, pulmonary TB).

With respect to the postcranial elements, slight PNBFs - frequently described as not specific but probable signs of pulmonary TB and/or TB pleurisy (e.g., Kelley \& Micozzi, 1984; Roberts et al., 1994; Santos \& Roberts, 2001, 2006; Maczel, 2003; Matos \& Santos, 2006; Giacon, 2008; Mariotti et al., 2015) - occurred on the vertebral end $\left(2^{\text {nd }}-4^{\text {th }}\right.$ and $7^{\text {th }}-$ $\left.8^{\text {th }}\right)$, and occasionally on the body $\left(2^{\text {nd }}-4^{\text {th }}\right)$ and/or sternal end $\left(3^{\text {rd }}-4^{\text {th }}\right)$ of five left side ribs $\left(2^{\text {nd }}-4^{\text {th }}\right.$ and $\left.7^{\text {th }}-8^{\text {th }}\right)$, as well as on the vertebral end of two right side ribs $\left(7^{\text {th }}-8^{\text {th }}\right)$, exclusively affecting the visceral surfaces. In the vertebral column, multiple, smooth-walled resorptive pits often connected by horizontal, superficial vascular channels were recognised on the lateral aspects of the thoracic (T3-12) and lumbar (L1-5) vertebral bodies, possibly referring to early-stage skeletal TB (e.g., Ménard, 1888; Baker, 1999; Maczel, 2003; Giacon, 2008; Mariotti et al., 2015). Although the observed endocranial and postcranial bony changes are not pathognomonic features of TB, the cause of death of Terry No. 1033 supports their tuberculous origin. 


\subsubsection{Periosteal appositions}

\begin{tabular}{|c|c|c|c|c|c|}
\hline \multicolumn{2}{|c|}{$\Sigma=427$} & \multicolumn{2}{|c|}{ TB group } & \multicolumn{2}{|c|}{ NTB group } \\
\hline \multicolumn{2}{|c|}{$\begin{array}{l}\text { Number of specimens } \\
\text { affected by PAs }\end{array}$} & \multicolumn{2}{|c|}{$\begin{array}{c}47 / 234 \\
(20.09 \%)\end{array}$} & \multicolumn{2}{|c|}{$\begin{array}{c}20 / 193 \\
(10.36 \%)\end{array}$} \\
\hline Females & Males & $\begin{array}{c}15 / 65 \\
(23.08 \%)\end{array}$ & $\begin{array}{c}32 / 169 \\
(18.93 \%)\end{array}$ & $\begin{array}{c}9 / 87 \\
(10.34 \%)\end{array}$ & $\begin{array}{c}11 / 106 \\
(10.38 \%)\end{array}$ \\
\hline
\end{tabular}

Table 3: Number of specimens exhibiting PAs in the TB group and NTB group by sex.

Periosteal appositions were observed in 67 of 427 (15.69\%) skeletons evaluated: of specimens identified to have died of TB, 20.09\% (47/234) showed PAs on the endocranial surface of the skull; whereas of individuals recorded to have died of causes other than TB, $10.36 \%(20 / 193)$ exhibited these lesions (Table 3). The $\chi^{2}$ comparison of the frequencies of PAs revealed a statistically significant difference between the two groups $\left(\chi^{2}=6.841, \mathrm{df}=1\right.$, $\mathrm{P}=0.0089$ ). Nevertheless, when the two groups were compared considering the sex (Table 3), there was no statistically significant difference between specimens with TB as the cause of death and individuals with NTB causes of death (females: $\chi^{2}=3.629, \mathrm{df}=1, \mathrm{P}=0.0568$; males: $\left.\chi^{2}=2.997, \mathrm{df}=1, \mathrm{P}=0.0834\right)$. The frequencies of PAs in females and males were very similar in both the TB group and NTB group (Table 3), and the $\chi^{2}$ testing of data revealed no statistically significant difference between the two sexes (TB group: $\chi^{2}=0.277, \mathrm{df}=1$, $\mathrm{P}=0.5987$; NTB group: $\chi^{2}=0.0529, \mathrm{df}=1, \mathrm{P}=0.8181$ ).

As for the distribution of affected specimens by age at death in the TB group, periosteal appositions occurred with the highest frequency among individuals between 20 and 29 years of age (25/56, 44.64\%) (Fig 29A): with 44.44\% (8/18) among females (Fig. 29B) and with $44.74 \%$ (17/38) among males (Fig. 29C). Among specimens under the age of 20 years, only an 18-year-old female revealed PAs on the inner surface of the skull (Fig. 29B); whereas among individuals above the age of 29 years - except for males between 30 and 39 years of age $(8 / 41,19.51 \%)$ (Fig. 29C) - PAs were registered only in a few cases both among females (Fig. 29B) and males (Fig. 29C). In contrast to the TB group, there was no specimen under the age of 30 years and exhibiting PAs on the inner surface of the skull in the NTB group (Fig. 30A-C). With the exception of a 37-year-old male and a 39-year-old female, individuals with NTB causes of death and PAs $(18 / 20,90.00 \%)$ were above the age of 40 years (Fig. 30A-C). The $\chi^{2}$ testing of the frequencies of PAs between various age groups was not assessed because of the low number of specimens in certain age groups. 
Figure 29: Demographic profile of specimens exhibiting PAs in the TB group:

A) total sample $(47 / 234,20.09 \%)$, B) females $(15 / 65,23.08 \%)$, and C) males $(32 / 169,18.93 \%)$.

A)



$\square$ Number of specimens $(\Sigma=234) \quad \square$ Number of specimens affected by PAs $(\Sigma=47)$

B)



$\square$ Number of females $(\Sigma=65) \quad \square$ Number of females affected by PAs $(\Sigma=15)$

C)



$\square$ Number of males $(\Sigma=169) \quad \square$ Number of males affected by PAs $(\Sigma=32)$ 
Figure 30: Demographic profile of specimens exhibiting PAs in the NTB group:

A) total sample $(20 / 193,10.36 \%)$, B) females $(9 / 87,10.34 \%)$, and C) males $(11 / 106,10.38 \%)$.

A)



$\square$ Number of specimens $(\Sigma=193) \quad \square$ Number of specimens affected by PAs $(\Sigma=20)$

B)



$\square$ Number of females $(\Sigma=87) \quad \square$ Number of females affected by PAs $(\Sigma=9)$

C)



$\square$ Number of males $(\Sigma=106) \quad \square$ Number of males affected by PAs $(\Sigma=11)$ 
Regarding the localisation of PAs on the inner surface of the skull, the frontal bone (predominantly its most protruding portions (Fig. 31A - blue) and/or orbital part (Fig. 31B - blue)) and the left and right parietal bones (particularly their most protruding portions and/or their parts along the superior sagittal sinus (Fig. 31A - blue)) represented the most common sites of involvement in both the TB group and NTB group (Table 4A-B). Nonetheless, less often the occipital bone (generally its squamous part (Fig. 31B - orange)), the left and right temporal bones (predominantly their squamous parts (Fig. 31B - orange)), and more rarely the left and right greater wings of the sphenoid bone (Fig. 31B - yellow) were also affected (Table $\mathbf{4 A}-\mathbf{B})$.

A)



B)



Figure 31: Typical localisations of PAs on the inner surface of the A) skullcap and B) skull base (blue: most commonly affected areas, orange: commonly affected areas, and yellow: less commonly affected areas) (drawings by Luca Kis).

Although the number of cranial bones concurrently involved by PAs (considering the left and right greater wings of the sphenoid bone as two separate bones) varied from one to eight in both groups, in 53.19\% (25/47) of individuals with TB as the cause of death and PAs, at least four cranial bones were simultaneously affected (Fig. 32A); whereas in nearly two-thirds $(13 / 20,65.00 \%)$ of specimens with NTB causes of death and PAs, less than four cranial bones were concomitantly involved (Fig. 32B). 
A)



B)

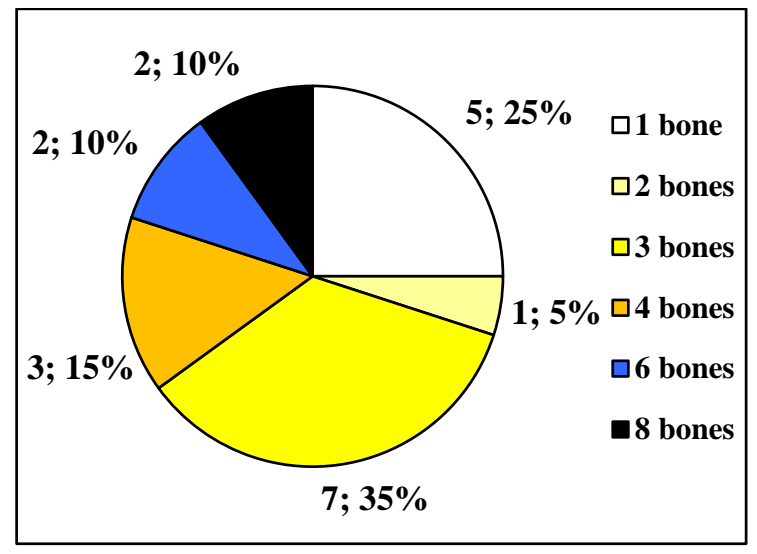

Figure 32: Distribution of specimens affected by PAs in the A) TB group ( $\Sigma=47)$ and

B) NTB group $(\Sigma=20)$ by number of simultaneously involved cranial bones (considering the left and right greater wings of the sphenoid bone as two separate bones).

With respect to the number of presented lesions in the TB group, the vast majority of PAs occurred as multifocal alterations on the inner surface of the skull in all cranial bones examined, except for the left and right greater wings of the sphenoid bone, where the ratio of multifocal and unifocal PAs was about 3:2 (Table 4A). Among individuals recorded to have died of causes other than TB, almost exclusively multifocal PAs were registered in all cranial bones evaluated (Table 4B). Concerning the extent of detected lesions, the majority of PAs observed in the TB group covered less than one-half of the endocranial surfaces in all cranial bones examined (Table 4A). However, the extent of PAs noted around the most protruding parts of the frontal and the left and right parietal bones, as well as in the squamous part of the left temporal bone, exceeded one-half of the inner surfaces quite often: in 20.59\% (7/34), 23.68\% (9/38), 20.00\% (7/35), and 22.22\% (4/18) of cases, respectively (Table 4A). Among specimens with NTB causes of death, only four PAs detected around the most protruding portions of the frontal and the left and right parietal bones covered more than one-half of the endocranial surfaces (Table 4B).

Among individuals with PAs in the TB group, the type of tuberculosis as the cause of death was not specified on the morgue record and/or death certificate in six cases (Suppl. table 1,3 ). In the remaining 41 cases - with the exception of two specimens identified to have died of TB meningitis and an individual recorded to have died of skeletal TB pulmonary TB (38 cases) was registered as the cause of death (Suppl. table 1, 3). Among specimens with PAs in the NTB group, cardiovascular problems (14 cases), pneumonia (three cases), cancer (one case), syphilis (one case), and appendicitis (one case) were recorded as NTB causes of death (Suppl. table 2, 4). 
Table 4: Distribution of specimens exhibiting PAs in the A) TB group and B) NTB group by affected cranial bones (considering the left and right greater wings of the sphenoid bone as two separate bones), extent, and number of lesions $(\mathrm{L}=$ left, $R=$ right).

\begin{tabular}{|c|c|c|c|c|c|c|c|c|c|}
\hline \multirow{2}{*}{\multicolumn{2}{|c|}{$\begin{array}{c}\text { A) } \\
\text { TB group }(\Sigma=234)\end{array}$}} & Frontal bone & $\begin{array}{l}\text { Parietal bone } \\
\text { (L) }\end{array}$ & $\begin{array}{l}\text { Parietal bone } \\
\text { (R) }\end{array}$ & $\begin{array}{l}\text { Temporal bone } \\
\text { (L) }\end{array}$ & $\begin{array}{l}\text { Temporal bone } \\
\text { (R) }\end{array}$ & $\begin{array}{l}\text { Sphenoid bone } \\
\text { (L) }\end{array}$ & $\begin{array}{l}\text { Sphenoid bone } \\
\text { (R) }\end{array}$ & Occipital bone \\
\hline & & $\begin{array}{c}34 / 47 \\
(72.34 \%)\end{array}$ & $\begin{array}{c}38 / 47 \\
(80.85 \%)\end{array}$ & $\begin{array}{c}35 / 47 \\
(74.47 \%)\end{array}$ & $\begin{array}{c}18 / 47 \\
(38.30 \%)\end{array}$ & $\begin{array}{c}20 / 47 \\
(42.55 \%)\end{array}$ & $\begin{array}{c}10 / 47 \\
(21.28 \%)\end{array}$ & $\begin{array}{c}13 / 47 \\
(27.66 \%)\end{array}$ & $\begin{array}{c}27 / 47 \\
(57.45 \%)\end{array}$ \\
\hline \multirow{4}{*}{  } & $x<25 \%$ & $\begin{array}{c}18 / 34 \\
(52.94 \%)\end{array}$ & $\begin{array}{c}23 / 38 \\
(60.53 \%)\end{array}$ & $\begin{array}{c}21 / 35 \\
(60.00 \%)\end{array}$ & $\begin{array}{c}10 / 18 \\
(55.56 \%)\end{array}$ & $\begin{array}{c}14 / 20 \\
(70.00 \%)\end{array}$ & $\begin{array}{c}7 / 10 \\
(70.00 \%)\end{array}$ & $\begin{array}{c}9 / 13 \\
(69.23 \%)\end{array}$ & $\begin{array}{c}24 / 27 \\
(88.89 \%)\end{array}$ \\
\hline & $25 \% \leq x<50 \%$ & $\begin{array}{c}9 / 34 \\
(26.47 \%)\end{array}$ & $\begin{array}{c}6 / 38 \\
(15.79 \%)\end{array}$ & $\begin{array}{c}7 / 35 \\
(20.00 \%)\end{array}$ & $\begin{array}{c}4 / 18 \\
(22.22 \%)\end{array}$ & $\begin{array}{c}3 / 20 \\
(15.00 \%)\end{array}$ & $\begin{array}{c}1 / 10 \\
(10.00 \%)\end{array}$ & $\begin{array}{c}3 / 13 \\
(23.08 \%)\end{array}$ & $\begin{array}{c}1 / 27 \\
(3.70 \%)\end{array}$ \\
\hline & $50 \% \leq x<75 \%$ & $\begin{array}{c}2 / 34 \\
(5.88 \%) \\
\end{array}$ & $\begin{array}{c}4 / 38 \\
(10.53 \%) \\
\end{array}$ & $\begin{array}{c}4 / 35 \\
(11.43 \%) \\
\end{array}$ & $\begin{array}{c}3 / 18 \\
(16.67 \%) \\
\end{array}$ & $\begin{array}{c}3 / 20 \\
(15.00 \%)\end{array}$ & - & $\begin{array}{c}1 / 13 \\
(7.69 \%) \\
\end{array}$ & $\begin{array}{c}1 / 27 \\
(3.70 \%) \\
\end{array}$ \\
\hline & $75 \% \leq x$ & $\begin{array}{c}5 / 34 \\
(14.71 \%) \\
\end{array}$ & $\begin{array}{c}5 / 38 \\
(13.16 \%) \\
\end{array}$ & $\begin{array}{c}3 / 35 \\
(8.57 \%) \\
\end{array}$ & $\begin{array}{c}1 / 18 \\
(5.56 \%) \\
\end{array}$ & - & $\begin{array}{c}2 / 10 \\
(20.00 \%)\end{array}$ & - & $\begin{array}{c}1 / 27 \\
(3.70 \%) \\
\end{array}$ \\
\hline \multirow{2}{*}{  } & Unifocal & $\begin{array}{c}1 / 34 \\
(2.94 \%)\end{array}$ & $\begin{array}{c}3 / 38 \\
(7.89 \%)\end{array}$ & $\begin{array}{c}4 / 35 \\
(11.43 \%)\end{array}$ & $\begin{array}{c}1 / 18 \\
(5.56 \%)\end{array}$ & $\begin{array}{c}2 / 20 \\
(10.00 \%)\end{array}$ & $\begin{array}{c}4 / 10 \\
(40.00 \%)\end{array}$ & $\begin{array}{c}5 / 13 \\
(38.46 \%)\end{array}$ & $\begin{array}{c}4 / 27 \\
(14.81 \%)\end{array}$ \\
\hline & Multifocal & $\begin{array}{c}33 / 34 \\
(97.06 \%) \\
\end{array}$ & $\begin{array}{c}\mathbf{3 5} / 38 \\
(\mathbf{9 2 . 1 1 \% )} \\
\end{array}$ & $\begin{array}{c}\mathbf{3 1 / 3 5} \\
(\mathbf{8 8 . 5 7 \%}) \\
\end{array}$ & $\begin{array}{c}17 / 18 \\
(94.44 \%) \\
\end{array}$ & $\begin{array}{c}18 / 20 \\
(90.00 \%) \\
\end{array}$ & $\begin{array}{c}6 / 10 \\
(60.00 \%) \\
\end{array}$ & $\begin{array}{c}8 / 13 \\
(61.54 \%) \\
\end{array}$ & $\begin{array}{c}23 / 27 \\
(85.19 \%) \\
\end{array}$ \\
\hline
\end{tabular}

\begin{tabular}{|c|c|c|c|c|c|c|c|c|c|}
\hline \multirow{2}{*}{\multicolumn{2}{|c|}{$\begin{array}{c}\text { B) } \\
\text { NTB group }(\Sigma=193)\end{array}$}} & Frontal bone & $\begin{array}{l}\text { Parietal bone } \\
\text { (L) }\end{array}$ & $\begin{array}{l}\text { Parietal bone } \\
\text { (R) }\end{array}$ & $\begin{array}{l}\text { Temporal bone } \\
\text { (L) }\end{array}$ & $\begin{array}{l}\text { Temporal bone } \\
\text { (R) }\end{array}$ & $\begin{array}{l}\text { Sphenoid bone } \\
\text { (L) }\end{array}$ & $\begin{array}{l}\text { Sphenoid bone } \\
\text { (R) }\end{array}$ & Occipital bone \\
\hline & & $\begin{array}{c}14 / 20 \\
(70.00 \%) \\
\end{array}$ & $\begin{array}{c}15 / 20 \\
(\mathbf{7 5 . 0 0 \% )} \\
\end{array}$ & $\begin{array}{c}15 / 20 \\
(75.00 \%) \\
\end{array}$ & $\begin{array}{c}4 / 20 \\
(20.00 \%)\end{array}$ & $\begin{array}{c}4 / 20 \\
(20.00 \%)\end{array}$ & $\begin{array}{c}2 / 20 \\
(10.00 \%)\end{array}$ & $\begin{array}{c}2 / 20 \\
(10.00 \%)\end{array}$ & $\begin{array}{c}12 / 20 \\
(60.00 \%)\end{array}$ \\
\hline \multirow{4}{*}{  } & $x<25 \%$ & $\begin{array}{c}8 / 14 \\
(57.14 \%) \\
\end{array}$ & $\begin{array}{c}14 / 15 \\
(93.33 \%) \\
\end{array}$ & $\begin{array}{c}12 / 15 \\
(80.00 \%)\end{array}$ & $\begin{array}{c}3 / 4 \\
(75.00 \%) \\
\end{array}$ & $\begin{array}{c}2 / 4 \\
(50.00 \%)\end{array}$ & $\begin{array}{c}1 / 2 \\
(50.00 \%)\end{array}$ & $\begin{array}{c}1 / 2 \\
(50.00 \%)\end{array}$ & $\begin{array}{c}10 / 12 \\
(83.33 \%)\end{array}$ \\
\hline & $25 \% \leq x<50 \%$ & $\begin{array}{c}5 / 14 \\
(35.71 \%) \\
\end{array}$ & - & $\begin{array}{c}1 / 15 \\
(6.67 \%) \\
\end{array}$ & $\begin{array}{c}1 / 4 \\
(25.00 \%) \\
\end{array}$ & $\begin{array}{c}2 / 4 \\
(50.00 \%) \\
\end{array}$ & $\begin{array}{c}1 / 2 \\
(50.00 \%)\end{array}$ & $\begin{array}{c}1 / 2 \\
(50.00 \%)\end{array}$ & $\begin{array}{c}2 / 12 \\
(16.67 \%) \\
\end{array}$ \\
\hline & $50 \% \leq x<75 \%$ & $\begin{array}{c}1 / 14 \\
(7.14 \%) \\
\end{array}$ & - & $\begin{array}{c}1 / 15 \\
(6.67 \%) \\
\end{array}$ & - & - & - & - & - \\
\hline & $75 \% \leq x$ & - & $\begin{array}{c}1 / 15 \\
(6.67 \%) \\
\end{array}$ & $\begin{array}{c}1 / 15 \\
(6.67 \%) \\
\end{array}$ & - & - & - & - & - \\
\hline \multirow{2}{*}{ 离 } & Unifocal & $\begin{array}{c}2 / 14 \\
(14.29 \%) \\
\end{array}$ & $\begin{array}{c}1 / 15 \\
(6.67 \%) \\
\end{array}$ & $\begin{array}{c}1 / 15 \\
(6.67 \%) \\
\end{array}$ & $\begin{array}{c}1 / 4 \\
(25.00 \%) \\
\end{array}$ & $\begin{array}{c}1 / 4 \\
(25.00 \%) \\
\end{array}$ & - & - & $\begin{array}{c}3 / 12 \\
(25.00 \%) \\
\end{array}$ \\
\hline & Multifocal & $\begin{array}{c}12 / 14 \\
(85.71 \%)\end{array}$ & $\begin{array}{c}14 / 15 \\
(93.33 \%)\end{array}$ & $\begin{array}{c}14 / 15 \\
(93.33 \%)\end{array}$ & $\begin{array}{c}3 / 4 \\
(75.00 \%)\end{array}$ & $\begin{array}{c}3 / 4 \\
(75.00 \%)\end{array}$ & $\begin{array}{c}2 / 2 \\
(100.00 \%)\end{array}$ & $\begin{array}{c}2 / 2 \\
(100.00 \%)\end{array}$ & $\begin{array}{c}9 / 12 \\
(75.00 \%)\end{array}$ \\
\hline
\end{tabular}


The skeletal remains of Terry No. 987 - a 23-year-old male whose recorded cause of death was pulmonary TB (Suppl. table 1) - exhibited numerous pathological bony changes probably resulted from tuberculosis. In the skull, unifocal GIs - described by Schultz (e.g., 1999, 2001, 2003) and Schultz \& Schmidt-Schultz (2015) as pathognomonic vestiges of TBM, since representing pressure atrophy of the tubercles that affect the dura mater - were observed on the squamous part of the occipital bone. Furthermore, not specific but probable signs of TBM, namely multifocal, small, serpentine branching ABVIs accompanied by PAs (e.g., Schultz, 1993, 1999, 2001, 2003), were recognised on the squamous part of the frontal and occipital (Fig. 33) bones.



Figure 33: Multifocal ABVIs accompanied by PAs on the squamous part of the occipital bone (Terry No. 987, 23-year-old, male, pulmonary TB).

The left (Fig. 18A (p. 42)) and right parietal bones, the squamous part of the left and right temporal bones, and the left and right greater wings of the sphenoid bone also revealed patches of PAs. Moreover, APDIs (very slight stage) affecting the squamous part of the frontal bone and the left and right parietal bones were recorded, indicating eICP possibly due to hydrocephalus that may be associated with TBM (e.g., Schultz, 1993, 2001, 2003).

In the postcranial skeleton, nine left side ribs $\left(3^{\text {rd }}-11^{\text {th }}\right)$ showed slight PNBFs on the visceral surface of the sternal end $\left(4^{\text {th }}-11^{\text {th }}\right)$, and occasionally on the body $\left(4^{\text {th }}-6^{\text {th }}\right.$ and $9^{\text {th }}-$ $\left.11^{\text {th }}\right)$ and/or vertebral end $\left(3^{\text {rd }}-7^{\text {th }}\right)$ that may represent signs of an inflammatory response secondary to pulmonary TB and/or TB pleurisy (e.g., Kelley \& Micozzi, 1984; Roberts et al., 1994; Santos \& Roberts, 2001, 2006; Maczel, 2003; Matos \& Santos, 2006; Giacon, 2008; Mariotti et al., 2015). Besides the ribs, the anterior and posterior surfaces of both 
humeri (predominantly the distal portion of the shaft), the anterior surface of both radii (particularly the distal portion of the shaft), the lateral surface of the right ulna (all along the shaft), the posterior, medial or lateral surfaces of three left side $\left(2^{\text {nd }}-4^{\text {th }}\right)$ and four right side $\left(2^{\text {nd }}-5^{\text {th }}\right)$ metacarpals (mainly the middle and distal portions of the shaft), the lateral surface of both femora (particularly the proximal portion of the shaft), the lateral surface of both tibiae (all along the shaft), the medial surface of both fibulae (predominantly the middle and distal portions of the shaft), and the plantar surface of both $5^{\text {th }}$ metatarsals (mainly the distal portion of the shaft) also exhibited slight PNBFs, probably referring to HPO associated with pulmonary TB (e.g., Mensforth et al., 1978; Kelly et al., 1991; Winland et al., 1997; Rothschild \& Rothschild, 1998, 1999; Santos \& Roberts, 2001; Hershkovitz et al., 2002; Assis et al., 2011). Based on the recorded cause of death of Terry No. 987, the most likely aetiology of the observed endocranial and postcranial bony changes is tuberculosis.

Similar to the previous case, the skeletal remains of Terry No. 1027 - a c. 41-yearold female whose morgue record states pulmonary TB as the cause of death (Suppl. table 1) - revealed endocranial bony changes probably resulted from TBM. The orbital part of the frontal bone, the left and right parietal bones along the squamous suture, the squamous part of the occipital and the left and right (Fig. 34) temporal bones, and the right greater wing of the sphenoid bone exhibited GIs that were described by Schultz (e.g., 1999, 2001, 2003) and Schultz \& Schmidt-Schultz (2015) as pathognomonic vestiges of TBM.



Figure 34: Multifocal GIs accompanied by PAs on the squamous part of the right temporal bone (Terry No. 1027, c. 41-year-old, female, pulmonary TB). 
Furthermore, non-specific signs of inflammatory-haemorrhagic processes of the meninges - namely multifocal, serpentine branching ABVIs and patches of PAs (e.g., Schultz, 1993, 1999, 2001, 2003) -, occurred on the squamous part of the frontal bone (Fig. 35), in the left and right parietal bones (Fig. 35), on the squamous part of the right temporal bone (Fig. 34), and on the right greater wing of the sphenoid bone. There were no other bony changes probably associated with TB in the skeleton. Based on the recorded cause of death of Terry No. 1027, the tuberculous origin of the observed lesions cannot be excluded.



Figure 35: Multifocal ABVIs and PAs covering the endocranial surface of the frontal, as well as the left and right parietal bones (Terry No. 1027, c. 41-year-old, female, pulmonary TB).

Both the cranial and postcranial remains of Terry No. 1300 - a 28-year-old male who had died of TB (Suppl. table 1) - showed different types of pathological bony changes that may be ascribed to tuberculosis. Concerning the skull, shallow APDIs (very slight stage) 
were recorded on the squamous part of the frontal bone, indicating eICP possibly due to tuberculous involvement of the CNS (e.g., Schultz, 1993, 2001, 2003). Moreover, nonspecific vestiges of inflammatory-haemorrhagic processes of the meninges - namely multifocal, small patches of PAs (e.g., Schultz, 1993, 1999, 2001, 2003) - were detected on the squamous and orbital (Fig. 36) parts of the frontal bone, along the sagittal suture in the left and right parietal bones, and on the squamous part of the left and right temporal bones.



Figure 36: Multifocal PAs on the orbital part of the frontal bone (Terry No. 1300, 28-year-old, male, TB).

Besides the endocranial alterations probably associated with TBM, slight PNBFs frequently described as not specific but probable signs of pulmonary TB and/or TB pleurisy (e.g., Kelley \& Micozzi, 1984; Roberts et al., 1994; Santos \& Roberts, 2001, 2006; Maczel, 2003; Matos \& Santos, 2006; Giacon, 2008; Mariotti et al., 2015) - were observed on the visceral surface of the sternal $\left(4^{\text {th }}-5^{\text {th }}\right)$ and/or vertebral $\left(5^{\text {th }}\right)$ end of two right side ribs $\left(4^{\text {th }}\right.$ $\left.5^{\text {th }}\right)$. In the vertebral column, signs of hypervascularisation were recognised on the lateral aspects of the lower thoracic (T7-12) and lumbar (L1-5) vertebral bodies, possibly referring to early-stage skeletal TB (e.g., Ménard, 1888; Baker, 1999; Maczel, 2003; Giacon, 2008; Mariotti et al., 2015). Although the registered endocranial and postcranial bony changes are not pathognomonic features of TB, the cause of death of Terry No. 1300 supports their tuberculous origin. 


\subsubsection{Abnormal blood vessel impressions}

\begin{tabular}{|c|c|c|c|c|c|}
\hline \multicolumn{2}{|c|}{$\Sigma=427$} & \multicolumn{2}{|c|}{ TB group } & \multicolumn{2}{|c|}{ NTB group } \\
\hline \multicolumn{2}{|c|}{$\begin{array}{c}\text { Number of specimens } \\
\text { affected by ABVIs }\end{array}$} & \multicolumn{2}{|c|}{$\begin{array}{c}50 / 234 \\
(21.37 \%)\end{array}$} & \multicolumn{2}{|c|}{$\begin{array}{c}12 / 193 \\
(6.22 \%)\end{array}$} \\
\hline Females & Males & $\begin{array}{c}20 / 65 \\
(30.77 \%)\end{array}$ & $\begin{array}{c}30 / 169 \\
(17.75 \%)\end{array}$ & $\begin{array}{c}6 / 87 \\
(6.90 \%)\end{array}$ & $\begin{array}{c}6 / 106 \\
(5.66 \%)\end{array}$ \\
\hline
\end{tabular}

Table 5: Number of specimens exhibiting ABVIs in the TB group and NTB group by sex.

From a total of 427 skeletons evaluated, 62 (14.52\%) exhibited ABVIs on the inner surface of the skull: $50(21.37 \%)$ of 234 individuals recorded to have died of TB and 12 (6.22\%) of 193 specimens identified to have died of causes other than TB (Table 5); thus, there was a statistically extremely significant difference in the frequencies of ABVIs between the TB group and NTB group $\left(\chi^{2}=18.357, \mathrm{df}=1, \mathrm{P}<0.0001\right)$. When the two groups were compared considering the sex (Table 5), the difference in the frequencies of ABVIs remained significant for both females $\left(\chi^{2}=13.317, \mathrm{df}=1, \mathrm{P}=0.0003\right)$ and males $\left(\chi^{2}=7.342\right.$, $\mathrm{df}=1, \mathrm{P}=0.0067)$. Furthermore, an approximately 13 percentage point difference in the frequencies of ABVIs between females and males was found in the TB group: $30.77 \%$ versus $17.75 \%$, respectively (Table 5). The $\chi^{2}$ comparison of the frequencies of ABVIs between the two sexes in the TB group (Table 5) revealed a statistically significant difference $\left(\chi^{2}=3.992, \mathrm{df}=1, \mathrm{P}=0.0457\right)$. Nevertheless, the frequencies of ABVIs among females and males were very similar in the NTB group (Table 5); thus, there was no statistically significant difference between the two sexes $\left(\chi^{2}=0.00295, \mathrm{df}=1, \mathrm{P}=0.9567\right)$.

As for the distribution of affected individuals by age at death, abnormal blood vessel impressions occurred with the highest frequency among specimens under the age of 30 years $(28 / 63,44.44 \%)$ in the TB group (Fig. 37A): with 59.09\% (13/22) among females (Fig. 37B) and with $36.59 \%$ (15/41) among males (Fig. 37C). With the exception of females between 40 and 49 years of age $(4 / 13,30.77 \%$ ) (Fig. 37B) and males between 30 and 39 years of age (9/41, 21.95\%) (Fig. 37C), ABVIs were observed only in a few cases among individuals above the age of 29 years (Fig. 37A). In contrast to the TB group, only a 22-year-old male exhibited ABVIs on the inner surface of the skull among specimens under the age of 30 years in the NTB group (Fig. 38A-C). The rest of the individuals with NTB causes of death and ABVIs (11/12, 91.67\%) were above the age of 40 years (Fig. 38A-C). The $\chi^{2}$ testing of the frequencies of ABVIs between various age groups was not assessed because of the low number of specimens in certain age groups. 
Figure 37: Demographic profile of specimens exhibiting ABVIs in the TB group:

A) total sample $(50 / 234,21.37 \%)$, B) females $(20 / 65,30.77 \%)$, and C) males $(30 / 169,17.75 \%)$.

A)



$\square$ Number of specimens $(\Sigma=234) \quad \square$ Number of specimens affected by ABVIs $(\Sigma=50)$

B)



$\square$ Number of females $(\Sigma=65) \quad \square$ Number of females affected by ABVIs $(\Sigma=20)$

C)



$\square$ Number of males $(\Sigma=169) \quad \square$ Number of males affected by ABVIs $(\Sigma=30)$ 
Figure 38: Demographic profile of specimens exhibiting ABVIs in the NTB group:

A) total sample $(12 / 193,6.22 \%)$, B) females $(6 / 87,6.90 \%)$, and C) males $(6 / 106,5.66 \%)$.

A)



$\square$ Number of specimens $(\Sigma=193) \quad \square$ Number of specimens affected by ABVIs $(\Sigma=12)$

B)

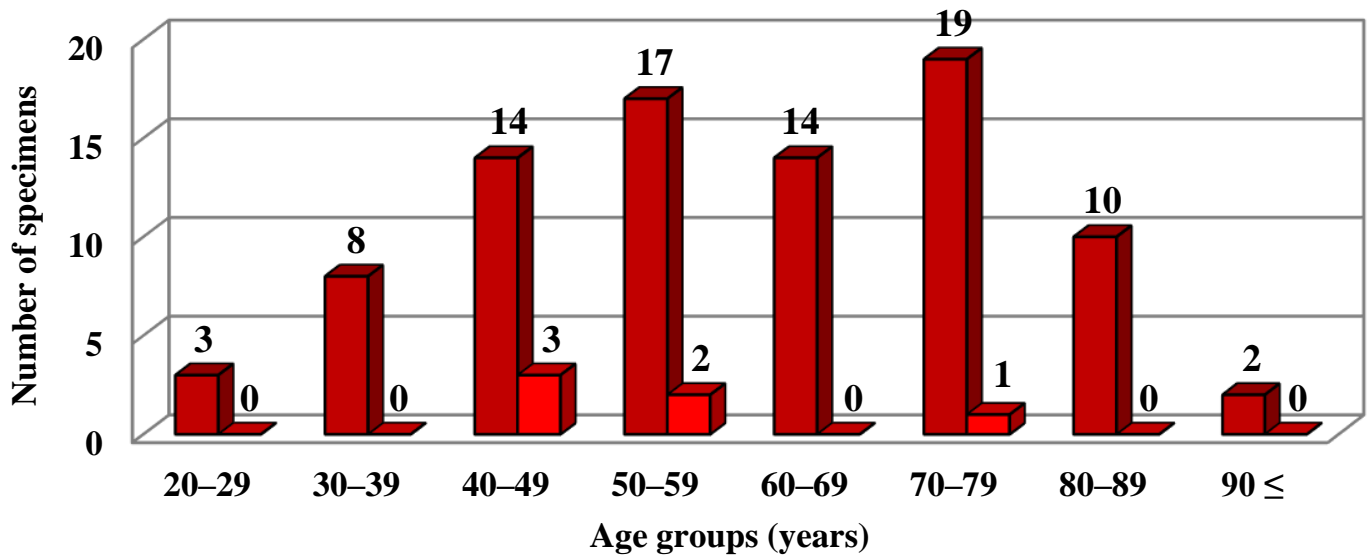

$\square$ Number of females $(\Sigma=87) \quad \square$ Number of females affected by ABVIs $(\Sigma=6)$

C)



$\square$ Number of males $(\Sigma=106) \quad \square$ Number of males affected by ABVIs $(\Sigma=6)$ 
Concerning the localisation of ABVIs, the frontal and the left and right parietal bones (particularly their most protruding portions and/or their parts along the superior sagittal sinus (Fig. 39A - blue)) represented the most common sites of involvement in both the TB group and NTB group (Table 6A-B). Occasionally, the involvement of the occipital bone (generally along the superior sagittal and/or transverse sinuses (Fig. 39B - orange)) was also registered among individuals with $\mathrm{TB}$ as the cause of death (Table 6A). With the exception of two cases showing ABVIs on the squamous part of the right temporal bone, and two further cases on the left and right greater wings of the sphenoid bone in the TB group (Fig. 39B - yellow), the left and right temporal bones, and the left and right greater wings of the sphenoid bone were not affected by these lesions both among specimens identified to have died of TB and individuals recorded to have died of causes other than TB (Table 6A-B).

A)



B)



Figure 39: Typical localisations of ABVIs on the inner surface of the A) skullcap and B) skull base (blue: most commonly affected areas, orange: commonly affected areas, and yellow: less commonly affected areas) (drawings by Luca Kis).

The number of cranial bones concurrently involved by ABVIs (considering the left and right greater wings of the sphenoid bone as two separate bones) varied from one to six in the TB group and from one to four in the NTB group. In nearly two-thirds (32/50, 64.00\%) of specimens with $\mathrm{TB}$ as the cause of death and ABVIs, at least three cranial bones were 
simultaneously affected (Fig. 40A); whereas in three-fourths $(9 / 12,75.00 \%)$ of individuals with NTB causes of death and ABVIs, less than three cranial bones were concomitantly involved (Fig. 40B).

A)



B)



Figure 40: Distribution of specimens affected by ABVIs in the A) TB group $(\Sigma=50)$ and B) NTB group $(\Sigma=12)$ by number of simultaneously involved cranial bones (considering the left and right greater wings of the sphenoid bone as two separate bones).

Regarding the number of presented lesions, ABVIs were almost exclusively detected as multifocal bony changes on the inner surface of the frontal, parietal, and occipital bones in both the TB group and NTB group (Table 6A-B). Nonetheless, unifocal ABVIs occurred in the left and right parietal, right temporal, and/or occipital bones in a few cases among both specimens identified to have died of TB and individuals recorded to have died of causes other than TB (Table 6A-B). With respect to the extent of registered lesions, the majority of observed ABVIs covered less than one-half of the endocranial surfaces in all cranial bones examined in both the TB group and NTB group (Table 6A-B). However, in the TB group, the extent of ABVIs noted in the frontal and the left and right parietal bones exceeded onehalf of the inner surfaces quite often: in $30.95 \%$ (13/42), 18.42\% (7/38), and 19.44\% (7/36) of cases, respectively (Table 6A).

Of 50 specimens with ABVIs in the TB group, 42 were identified to have died of pulmonary TB (Suppl. table 1, 3). Five additional individuals died of other types of tuberculosis, such as peritoneal TB (three cases), TB meningitis (one case), and miliary TB (one case); whereas in the remaining three cases, the type of tuberculosis as the cause of death was not specified on the morgue record and/or death certificate (Suppl. table 1, 3). In the NTB group, the most frequently registered NTB causes of death were cardiovascular problems, followed by respiratory diseases, syphilis, and different types of cancer among specimens exhibiting ABVIs on the inner surface of the skull (Suppl. table 2, 4). 
Table 6: Distribution of specimens exhibiting ABVIs in the A) TB group and B) NTB group by affected cranial bones (considering the left and right greater wings of the sphenoid bone as two separate bones), extent, and number of lesions $(\mathrm{L}=$ left, $R=$ right).

\begin{tabular}{|c|c|c|c|c|c|c|c|c|c|}
\hline & A) & Frontal bone & $\begin{array}{c}\text { Parietal bone } \\
\text { (L) }\end{array}$ & $\begin{array}{l}\text { Parietal bone } \\
\text { (R) }\end{array}$ & $\begin{array}{c}\text { Temporal bone } \\
\text { (L) }\end{array}$ & $\begin{array}{l}\text { Temporal bone } \\
\text { (R) }\end{array}$ & $\begin{array}{c}\text { Sphenoid bone } \\
\text { (L) }\end{array}$ & $\begin{array}{c}\text { Sphenoid bone } \\
\text { (R) }\end{array}$ & Occipital bone \\
\hline TB & oup $(\Sigma=234)$ & $\begin{array}{c}42 / 50 \\
(84.00 \%)\end{array}$ & $\begin{array}{c}38 / 50 \\
(76.00 \%)\end{array}$ & $\begin{array}{c}36 / 50 \\
(72.00 \%)\end{array}$ & $\begin{array}{c}\mathbf{0} / \mathbf{5 0} \\
(\mathbf{0 . 0 0 \%})\end{array}$ & $\begin{array}{c}2 / 50 \\
(4.00 \%)\end{array}$ & $\begin{array}{c}1 / 50 \\
(2.00 \%)\end{array}$ & $\begin{array}{c}1 / 50 \\
(2.00 \%)\end{array}$ & $\begin{array}{c}16 / 50 \\
(32.00 \%)\end{array}$ \\
\hline$\tilde{0}$ & $x<25 \%$ & $\begin{array}{c}13 / 42 \\
(30.95 \%)\end{array}$ & $\begin{array}{c}27 / 38 \\
(71.05 \%)\end{array}$ & $\begin{array}{c}23 / 36 \\
(63.89 \%)\end{array}$ & - & $\begin{array}{c}1 / 2 \\
(50.00 \%)\end{array}$ & - & - & $\begin{array}{c}15 / 16 \\
(93.75 \%)\end{array}$ \\
\hline$\frac{a}{0}$ & $25 \% \leq x<50 \%$ & $\begin{array}{c}16 / 42 \\
(38.10 \%)\end{array}$ & $\begin{array}{c}4 / 38 \\
(10.53 \%)\end{array}$ & $\begin{array}{c}6 / 36 \\
(16.67 \%)\end{array}$ & - & - & - & - & $\begin{array}{c}1 / 16 \\
(6.25 \%)\end{array}$ \\
\hline  & $50 \% \leq x<75 \%$ & $\begin{array}{c}7 / 42 \\
(16.67 \%)\end{array}$ & $\begin{array}{c}5 / 38 \\
(13.16 \%)\end{array}$ & $\begin{array}{c}5 / 36 \\
(13.89 \%)\end{array}$ & - & $\begin{array}{c}1 / 2 \\
(50.00 \%)\end{array}$ & $\begin{array}{c}1 / 1 \\
(100.00 \%)\end{array}$ & - & - \\
\hline 竎 & $75 \% \leq x$ & $\begin{array}{c}6 / 42 \\
(14.29 \%)\end{array}$ & $\begin{array}{c}2 / 38 \\
(5.26 \%)\end{array}$ & $\begin{array}{c}2 / 36 \\
(5.56 \%)\end{array}$ & - & - & - & $\begin{array}{c}1 / 1 \\
(100.00 \%)\end{array}$ & - \\
\hline อ̆ & Unifocal & - & $\begin{array}{c}2 / 38 \\
(5.26 \%)\end{array}$ & $\begin{array}{c}2 / 36 \\
(5.56 \%)\end{array}$ & - & $\begin{array}{c}2 / 2 \\
(100.00 \%)\end{array}$ & $\begin{array}{c}1 / 1 \\
(100.00 \%)\end{array}$ & $\begin{array}{c}1 / 1 \\
(100.00 \%)\end{array}$ & $\begin{array}{c}3 / 16 \\
(18.75 \%)\end{array}$ \\
\hline$\Xi^{\Xi}$ 离 & Multifocal & $\begin{array}{c}42 / 42 \\
(100.00 \%)\end{array}$ & $\begin{array}{c}36 / 38 \\
(94.74 \%)\end{array}$ & $\begin{array}{c}34 / 36 \\
(94.44 \%)\end{array}$ & - & - & - & - & $\begin{array}{c}13 / 16 \\
(81.25 \%)\end{array}$ \\
\hline
\end{tabular}

\begin{tabular}{|c|c|c|c|c|c|c|c|c|c|}
\hline \multirow{2}{*}{\multicolumn{2}{|c|}{ BTB group $(\Sigma=193)$}} & Frontal bone & $\begin{array}{c}\text { Parietal bone } \\
\text { (L) }\end{array}$ & $\begin{array}{c}\text { Parietal bone } \\
\text { (R) }\end{array}$ & $\begin{array}{c}\text { Temporal bone } \\
\text { (L) }\end{array}$ & $\begin{array}{l}\text { Temporal bone } \\
\text { (R) }\end{array}$ & $\begin{array}{c}\text { Sphenoid bone } \\
\text { (L) }\end{array}$ & $\begin{array}{l}\text { Sphenoid bone } \\
\text { (R) }\end{array}$ & Occipital bone \\
\hline & & $\begin{array}{c}5 / 12 \\
(41.67 \%)\end{array}$ & $\begin{array}{c}9 / 12 \\
(75.00 \%)\end{array}$ & $\begin{array}{c}10 / 12 \\
(83.33 \%)\end{array}$ & $\begin{array}{c}0 / 12 \\
(0.00 \%)\end{array}$ & $\begin{array}{c}0 / 12 \\
(0.00 \%)\end{array}$ & $\begin{array}{c}0 / 12 \\
(0.00 \%)\end{array}$ & $\begin{array}{c}0 / 12 \\
(0.00 \%)\end{array}$ & $\begin{array}{c}1 / 12 \\
(8.33 \%)\end{array}$ \\
\hline \multirow{4}{*}{  } & $x<25 \%$ & $\begin{array}{c}2 / 5 \\
(40.00 \%)\end{array}$ & $\begin{array}{c}6 / 9 \\
(66.67 \%)\end{array}$ & $\begin{array}{c}7 / 10 \\
(70.00 \%)\end{array}$ & - & - & - & - & $\begin{array}{c}1 / 1 \\
(100.00 \%)\end{array}$ \\
\hline & $25 \% \leq x<50 \%$ & $\begin{array}{c}2 / 5 \\
(40.00 \%)\end{array}$ & $\begin{array}{c}2 / 9 \\
(22.22 \%)\end{array}$ & $\begin{array}{c}2 / 10 \\
(20.00 \%)\end{array}$ & - & - & - & - & - \\
\hline & $50 \% \leq x<75 \%$ & $\begin{array}{c}1 / 5 \\
(20.00 \%)\end{array}$ & $\begin{array}{c}1 / 9 \\
(11.11 \%)\end{array}$ & $\begin{array}{c}1 / 10 \\
(10.00 \%)\end{array}$ & - & - & - & - & - \\
\hline & $75 \% \leq x$ & - & - & - & - & - & - & - & - \\
\hline \multirow{2}{*}{$\frac{\grave{d}}{\bar{\Xi}}$} & Unifocal & - & $\begin{array}{c}1 / 9 \\
(11.11 \%)\end{array}$ & $\begin{array}{c}1 / 10 \\
(10.00 \%)\end{array}$ & - & - & - & - & - \\
\hline & Multifocal & $\begin{array}{c}5 / 5 \\
(100.00 \%)\end{array}$ & $\begin{array}{c}8 / 9 \\
(88.89 \%)\end{array}$ & $\begin{array}{c}9 / 10 \\
(90.00 \%)\end{array}$ & - & - & - & - & $\begin{array}{c}1 / 1 \\
(100.00 \%)\end{array}$ \\
\hline
\end{tabular}


In the skeleton of Terry No. 254 - a 21-year-old male recorded to have died of pulmonary TB (Suppl. table 1) -, pathological bony changes that may be attributed to tuberculosis were registered both in the cranial and postcranial elements. Concerning the skull, deep APDIs (pronounced stage) were noted all over the inner surface of the skullcap and skull base, referring to eICP possibly due to tuberculous involvement of the CNS (e.g., Schultz, 1993, 2001, 2003). Furthermore, non-specific vestiges of haemorrhagic and/or inflammatory meningeal reactions - namely multifocal, small, serpentine branching ABVIs accompanied by slight PAs (e.g., Schultz, 1993, 1999, 2001, 2003) - were detected in the deep DIs located in the frontal (Fig. 19C (p. 44)) and parietal (Fig. 41) bones, covering about three-fourths (stage 4) of the inner surfaces. Moreover, the endocranial surface of the squamous part of the temporal bones and the right greater wing of the sphenoid bone also exhibited small patches of PAs.



Figure 41: Multifocal ABVIs accompanied by PAs along the squamous suture in the right parietal bone (Terry No. 254, 21-year-old, male, pulmonary TB).

Besides the endocranial alterations probably associated with TBM, very slight PNBFs - frequently described as not specific but probable signs of pulmonary TB and/or TB pleurisy (e.g., Kelley \& Micozzi, 1984; Roberts et al., 1994; Santos \& Roberts, 2001, 2006; Maczel, 2003; Matos \& Santos, 2006; Giacon, 2008; Mariotti et al., 2015) - occurred on the sternal end (left $3^{\text {rd }}-6^{\text {th }}$, right $2^{\text {nd }}-3^{\text {rd }}$ and $6^{\text {th }}-8^{\text {th }}$ ), and occasionally on the body (right $3^{\text {rd }}-$ $\left.4^{\text {th }}\right)$ and vertebral end (right $\left.2^{\text {nd }}-3^{\text {rd }}\right)$ of four left side $\left(3^{\text {rd }}-6^{\text {th }}\right)$ and seven right side $\left(2^{\text {nd }}-8^{\text {th }}\right)$ ribs, exclusively affecting the visceral surfaces. Slight PNBFs were also recorded on the anterior surface of both humeri and radii (predominantly on the distal portion of the shaft), on the posterior surface of the right ulna (all along the shaft), in the supraspinatous fossa of 
both scapulae, and on the medial surface of both calcanei, indicating HPO probably related to pulmonary TB (e.g., Mensforth et al., 1978; Kelly et al., 1991; Winland et al., 1997; Rothschild \& Rothschild, 1998, 1999; Santos \& Roberts, 2001; Hershkovitz et al., 2002; Assis et al., 2011). Although the observed endocranial and postcranial bony changes are not pathognomonic features of TB, the cause of death of Terry No. 254 supports their tuberculous origin.

Similar to the previous case, the skeletal remains of Terry No. 1030 - a 62-year-old male whose death certificate states pulmonary TB as the cause of death (Suppl. table 1) revealed endocranial bony changes probably resulted from TBM. Multifocal, serpentine branching ABVIs - described by Schultz (e.g., 1993, 1999, 2001, 2003) as non-specific vestiges of inflammatory-haemorrhagic processes of the meninges - occurred on the inner surface of the frontal and the left and right parietal (Fig. 42A) bones, predominantly along the sagittal sinus. The detected lesions covered more than three-fourths (stage 4) of the inner surfaces. The left and right (Fig. 42B) greater wings of the sphenoid bone and the squamous part of the occipital bone (Fig. 43) (particularly along the sagittal and transverse sinuses) also showed ABVIs. There were no other bony changes probably associated with TB in the skeleton. Based on the recorded cause of death of Terry No. 1030, the tuberculous origin of the observed ABVIs cannot be excluded.

A)



B)

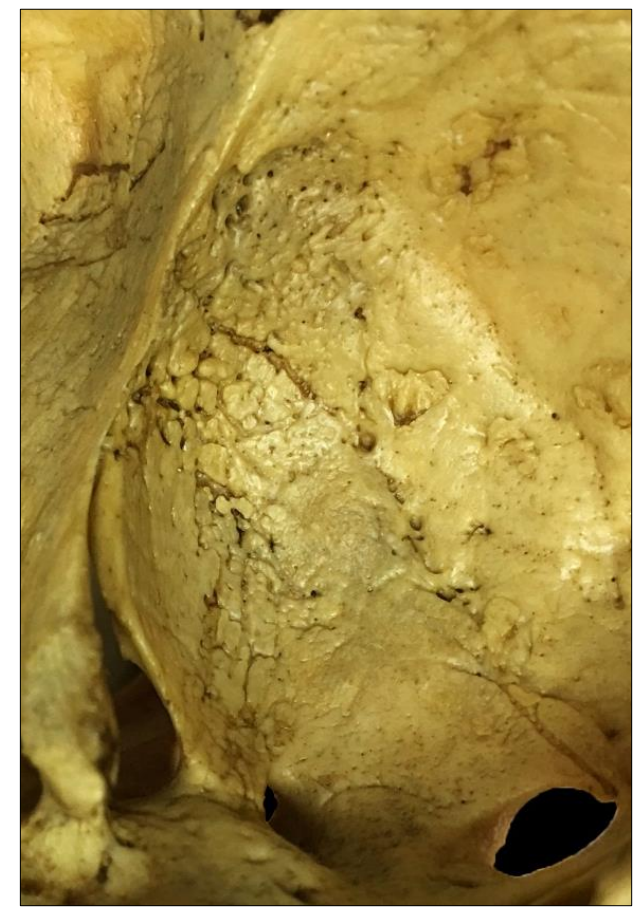

Figure 42: ABVIs A) along the sagittal sinus in the parietal bones and B) on the right greater wing of the sphenoid bone (Terry No. 1030, 62-year-old, male, pulmonary TB). 




Figure 43: ABVIs along the sagittal and transverse sinuses on the squamous part of the occipital bone (Terry No. 1030, 62-year-old, male, pulmonary TB).

Both the cranial and postcranial remains of Terry No. 1555 - a 41-year-old female who had died of pulmonary TB (Suppl. table 1) - showed different types of pathological bony changes that may be ascribed to tuberculosis. In the skull, shallow APDIs (very slight stage) affecting the squamous part of the frontal and the left and right parietal bones were recorded, referring to eICP possibly due to hydrocephalus that may be related to TBM (e.g., Schultz, 1993, 2001, 2003). Non-specific signs of the inflammatory-haemorrhagic meningeal processes, namely ABVIs (e.g., Schultz, 1993, 1999, 2001, 2003), were noted on the endocranial surface of the frontal and the left and right parietal bones, particularly around their most protruding portions (Fig. 44).

As for the postcranial skeleton, five left $\left(1^{\text {st }}-5^{\text {th }}\right)$ and three right $\left(2^{\text {nd }}-4^{\text {th }}\right)$ side ribs showed slight PNBFs on the visceral surface of the vertebral end that may represent vestiges of an inflammatory response secondary to pulmonary TB and/or TB pleurisy (e.g., Kelley \& Micozzi, 1984; Roberts et al., 1994; Santos \& Roberts, 2001, 2006; Maczel, 2003; Matos \& Santos, 2006; Giacon, 2008; Mariotti et al., 2015). Besides the ribs, the medial and lateral surfaces of both tibiae (predominantly the proximal and distal portions of the shaft), the anterior and posterior surfaces of both fibulae (particularly the middle portion of the shaft), the medial surface of both calcanei, the anterior and medial surfaces of both radii (mainly 
the distal portion of the shaft), the supraspinatous fossa of both scapulae, and the palmar surface of both $1^{\text {st }}$ metacarpals (particularly the proximal portion of the shaft) also exhibited slight PNBFs, indicating HPO probably associated with pulmonary TB (e.g., Mensforth et al., 1978; Kelly et al., 1991; Winland et al., 1997; Rothschild \& Rothschild, 1998, 1999; Santos \& Roberts, 2001; Hershkovitz et al., 2002; Assis et al., 2011). Based on the recorded cause of death of Terry No. 1555, the most likely aetiology of the observed endocranial and postcranial bony changes is tuberculosis.



Figure 44: ABVIs on the endocranial surface of the frontal and parietal bones, predominantly affecting the most protruding portions (Terry No. 1555, 41-year-old, female, pulmonary TB). 


\subsubsection{Granular impressions}

\begin{tabular}{|c|c|c|c|c|c|}
\hline \multicolumn{2}{|c|}{$\Sigma=427$} & \multicolumn{2}{|c|}{ TB group } & \multicolumn{2}{|c|}{ NTB group } \\
\hline \multicolumn{2}{|c|}{$\begin{array}{l}\text { Number of specimens } \\
\text { affected by GIs }\end{array}$} & \multicolumn{2}{|c|}{$\begin{array}{c}68 / 234 \\
(29.06 \%)\end{array}$} & \multicolumn{2}{|c|}{$\begin{array}{c}6 / 193 \\
(3.11 \%)\end{array}$} \\
\hline Females & Males & $\begin{array}{c}20 / 65 \\
(30.77 \%)\end{array}$ & $\begin{array}{c}48 / 169 \\
(28.40 \%)\end{array}$ & $\begin{array}{c}2 / 87 \\
(2.30 \%)\end{array}$ & $\begin{array}{c}4 / 106 \\
(3.77 \%)\end{array}$ \\
\hline
\end{tabular}

Table 7: Number of specimens exhibiting GIs in the TB group and NTB group by sex.

GIs were detected in $17.33 \%(74 / 427)$ of skeletons examined: in $29.06 \%(68 / 234)$ of the TB group and in $3.11 \%(6 / 193)$ of the NTB group (Table 7). The $\chi^{2}$ testing of the frequencies of GIs in specimens with TB as the cause of death and individuals with NTB causes of death revealed a statistically extremely significant difference between the two groups $\left(\chi^{2}=47.922, \mathrm{df}=1, \mathrm{P}<0.0001\right)$. When the two groups were compared considering the sex (Table 7), the difference in the frequencies of GIs remained extremely significant for both females $\left(\chi^{2}=22.115, \mathrm{df}=1, \mathrm{P}<0.0001\right)$ and males $\left(\chi^{2}=24.188, \mathrm{df}=1, \mathrm{P}<0.0001\right)$. However, the frequencies of GIs in females and males were very similar in both groups (Table 7); thus, there was no statistically significant difference between the two sexes (TB group: $\chi^{2}=0.0386, \mathrm{df}=1, \mathrm{P}=0.8443$; NTB group: $\chi^{2}=0.0291, \mathrm{df}=1, \mathrm{P}=0.8645$ ).

With respect to the distribution of affected specimens by age at death in the TB group, granular impressions occurred with the highest frequency among individuals under the age of 20 years (4/7, 57.14\%) (Fig. 45A): with 50.00\% (2/4) among females (Fig. 45B) and with $66.67 \%$ (2/3) among males (Fig. 45C). Nevertheless, more than one-third (55/158, 34.81\%) of specimens between 20 and 49 years of age and recorded to have died of TB also exhibited GIs on the endocranial surface of the skull (females: 17/43, 39.53\%; males: 38/115, 33.04\%) (Fig. 45A-C). Except for males between 50 and 59 years of age $(5 / 27,18.52 \%)$ (Fig. 45C), GIs occurred only in a few cases among individuals above the age of 49 years: in a 73-yearold female, in two males between 60 and 69 years of age, and in a 71-year-old male (Fig. 45A-C). In the NTB group, with the exception of a 25 -year-old male, specimens affected by GIs were above the age of 40 years: a female and two males between 40 and 49 years of age, a 54-year-old male, and a 60-year-old female (Fig. 46A-C). The $\chi^{2}$ testing of the frequencies of GIs between various age groups was not assessed because of the low number of individuals in certain age groups. 
Figure 45: Demographic profile of specimens exhibiting GIs in the TB group:

A) total sample $(68 / 234,29.06 \%)$, B) females $(20 / 65,30.77 \%)$, and C) males $(48 / 169,28.40 \%)$.

A)



$\square$ Number of specimens $(\Sigma=234) \quad \square$ Number of specimens affected by GIs $(\Sigma=68)$

B)



$\square$ Number of females $(\Sigma=65) \quad \square$ Number of females affected by GIs $(\Sigma=20)$

C)



$\square$ Number of males $(\Sigma=169) \quad \square$ Number of males affected by GIs $(\Sigma=48)$ 
Figure 46: Demographic profile of specimens exhibiting GIs in the NTB group:

A) total sample $(6 / 193,3.11 \%)$, B) females $(2 / 87,2.30 \%)$, and C) males $(4 / 106,3.77 \%)$.

A)



$\square$ Number of specimens ( $\Sigma=193) \quad \square$ Number of specimens affected by GIs $(\Sigma=6)$

B)



- Number of females $(\Sigma=87) \quad \square$ Number of females affected by GIs $(\Sigma=2)$

C)



Age groups (years)

$\square$ Number of males $(\Sigma=106)$

$\square$ Number of males affected by GIs $(\Sigma=4)$ 
Concerning the localisation of GIs, the most commonly affected area of the inner surface of the skull was the squamous part of the occipital bone (Fig. 47 - blue) in both the TB group and NTB group (Table 8A-B). Furthermore, GIs were quite often observed in the orbital part of the frontal bone and in the squamous part of the left and right temporal bones (Fig. 47 - orange; Table 8A-B). Occasionally, the involvement of the left and right greater wings of the sphenoid bone, as well as the left and right parietal bones (predominantly along the squamous suture), was also registered (Fig. 47 - yellow; Table 8A-B).



Figure 47: Typical localisations of GIs on the inner surface of the skull base (blue: most commonly affected areas, orange: commonly affected areas, and yellow: less commonly affected areas) (drawing by Luca Kis).

In both groups, less than four cranial bones (considering the left and right greater wings of the sphenoid bone as two separate bones) were simultaneously affected by GIs in approximately two-thirds of individuals (TB group: 49/68, 72.06\%; NTB group: 4/6, 66.67\%) (Fig. 48A-B). 
A)



B)

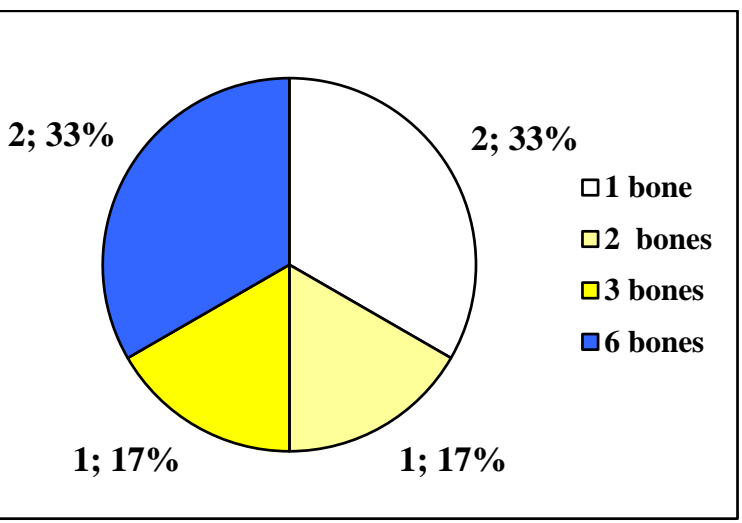

Figure 48: Distribution of specimens affected by GIs in the A) TB group $(\Sigma=68)$ and B) NTB group $(\Sigma=6)$ by number of simultaneously involved cranial bones (considering the left and right greater wings of the sphenoid bone as two separate bones).

Regarding the number of presented lesions among specimens identified to have died of TB, GIs were particularly recorded as multifocal bony changes in the occipital and frontal bones, as unifocal alterations on the left and right greater wings of the sphenoid bone and in the left and right parietal bones; whereas the frequencies of unifocal and multifocal GIs were similar in the left and right temporal bones (Table 8A). Among individuals recorded to have died of causes other than TB, only two GIs involving the left and right greater wings of the sphenoid bone were registered as unifocal alterations (Table 8B). As for the extent of detected lesions, the majority of GIs observed in the TB group covered less than one-fourth of the endocranial surfaces in all cranial bones examined (Table 8A). Nonetheless, the extent of GIs in the squamous part of the right temporal bone and on the right greater wing of the sphenoid bone exceeded one-fourth of the inner surfaces quite often: in $30.00 \%(6 / 20)$ and $42.11 \%$ (8/19) of cases, respectively (Table 8A). In the NTB group, only three GIs detected on the left and right greater wings of the sphenoid bone and in the squamous part of the occipital bone covered more than one-fourth of the endocranial surfaces (Table 8B).

From a total of 68 individuals with GIs in the TB group, 53 died of pulmonary TB (Suppl. table 1, 3). Five additional specimens died of other types of tuberculosis, such as skeletal TB (three cases), TB meningitis (one case), and peritoneal TB (one case); whereas in the remaining ten cases, the type of tuberculosis as the cause of death was not specified on the morgue record and/or death certificate (Suppl. table 1, 3). Among the NTB causes of death of individuals with GIs, cardiovascular problems (three cases), cancer (two cases), and peritonitis (one case) were recorded (Suppl. table 2, 4). 
Table 8: Distribution of specimens exhibiting GIs in the A) TB group and B) NTB group by affected cranial bones (considering the left and right greater wings of the sphenoid bone as two separate bones), extent, and number of lesions $(\mathrm{L}=$ left, $R=$ right).

\begin{tabular}{|c|c|c|c|c|c|c|c|c|c|}
\hline \multirow{2}{*}{\multicolumn{2}{|c|}{$\begin{array}{c}\text { A) } \\
\text { TB group }(\Sigma=234)\end{array}$}} & Frontal bone & $\begin{array}{c}\text { Parietal bone } \\
\text { (L) }\end{array}$ & $\begin{array}{l}\text { Parietal bone } \\
\text { (R) }\end{array}$ & $\begin{array}{c}\text { Temporal bone } \\
\text { (L) }\end{array}$ & $\begin{array}{l}\text { Temporal bone } \\
\text { (R) }\end{array}$ & $\begin{array}{l}\text { Sphenoid bone } \\
\text { (L) }\end{array}$ & $\begin{array}{l}\text { Sphenoid bone } \\
\text { (R) }\end{array}$ & Occipital bone \\
\hline & & $\begin{array}{c}32 / 68 \\
(47.06 \%)\end{array}$ & $\begin{array}{c}6 / 68 \\
(8.82 \%)\end{array}$ & $\begin{array}{c}10 / 68 \\
(14.71 \%)\end{array}$ & $\begin{array}{c}20 / 68 \\
(29.41 \%)\end{array}$ & $\begin{array}{c}20 / 68 \\
(29.41 \%)\end{array}$ & $\begin{array}{c}10 / 68 \\
(14.71 \%)\end{array}$ & $\begin{array}{c}19 / 68 \\
(27.94 \%)\end{array}$ & $\begin{array}{c}62 / 68 \\
(91.18 \%)\end{array}$ \\
\hline \multirow{4}{*}{  } & $x<25 \%$ & $\begin{array}{c}31 / 32 \\
(96.88 \%) \\
\end{array}$ & $\begin{array}{c}6 / 6 \\
(100.00 \%)\end{array}$ & $\begin{array}{c}10 / 10 \\
(100.00 \%)\end{array}$ & $\begin{array}{c}18 / 20 \\
(90.00 \%)\end{array}$ & $\begin{array}{c}14 / 20 \\
(70.00 \%)\end{array}$ & $\begin{array}{c}9 / 10 \\
(90.00 \%)\end{array}$ & $\begin{array}{c}11 / 19 \\
(57.89 \%)\end{array}$ & $\begin{array}{c}50 / 62 \\
(80.65 \%)\end{array}$ \\
\hline & $25 \% \leq x<50 \%$ & $\begin{array}{c}1 / 32 \\
(3.13 \%) \\
\end{array}$ & - & - & $\begin{array}{c}2 / 20 \\
(10.00 \%)\end{array}$ & $\begin{array}{c}2 / 20 \\
(10.00 \%)\end{array}$ & $\begin{array}{c}1 / 10 \\
(10.00 \%)\end{array}$ & $\begin{array}{c}7 / 19 \\
(36.84 \%) \\
\end{array}$ & $\begin{array}{c}11 / 62 \\
(17.74 \%) \\
\end{array}$ \\
\hline & $50 \% \leq x<75 \%$ & - & - & - & - & $\begin{array}{c}4 / 20 \\
(20.00 \%) \\
\end{array}$ & - & $\begin{array}{c}1 / 19 \\
(5.26 \%) \\
\end{array}$ & $\begin{array}{c}1 / 62 \\
(1.61 \%) \\
\end{array}$ \\
\hline & $75 \% \leq x$ & - & - & - & - & - & - & - & - \\
\hline \multirow{2}{*}{$\frac{\grave{\Xi}}{\bar{E}}$} & Unifocal & $\begin{array}{c}8 / 32 \\
(25.00 \%)\end{array}$ & $\begin{array}{c}4 / 6 \\
(66.67 \%)\end{array}$ & $\begin{array}{c}6 / 10 \\
(60.00 \%)\end{array}$ & $\begin{array}{c}9 / 20 \\
(45.00 \%)\end{array}$ & $\begin{array}{c}9 / 20 \\
(45.00 \%)\end{array}$ & $\begin{array}{c}9 / 10 \\
(90.00 \%)\end{array}$ & $\begin{array}{c}14 / 19 \\
(73.68 \%)\end{array}$ & $\begin{array}{c}9 / 62 \\
(14.52 \%)\end{array}$ \\
\hline & Multifocal & $\begin{array}{c}24 / 32 \\
(\mathbf{7 5 . 0 0 \%}) \\
\end{array}$ & $\begin{array}{c}2 / 6 \\
(33.33 \%) \\
\end{array}$ & $\begin{array}{c}4 / 10 \\
(40.00 \%) \\
\end{array}$ & $\begin{array}{c}11 / 20 \\
(55.00 \%)\end{array}$ & $\begin{array}{c}11 / 20 \\
(55.00 \%)\end{array}$ & $\begin{array}{c}1 / 10 \\
(10.00 \%)\end{array}$ & $\begin{array}{c}5 / 19 \\
(26.32 \%) \\
\end{array}$ & $\begin{array}{c}53 / 62 \\
(85.48 \%) \\
\end{array}$ \\
\hline
\end{tabular}

\begin{tabular}{|c|c|c|c|c|c|c|c|c|c|}
\hline & B) & Frontal bone & $\begin{array}{c}\text { Parietal bone } \\
\text { (L) }\end{array}$ & $\begin{array}{c}\text { Parietal bone } \\
\text { (R) }\end{array}$ & $\begin{array}{c}\text { Temporal bone } \\
\text { (L) }\end{array}$ & $\begin{array}{c}\text { Temporal bone } \\
\text { (R) }\end{array}$ & $\begin{array}{l}\text { Sphenoid bone } \\
\text { (L) }\end{array}$ & $\begin{array}{c}\text { Sphenoid bone } \\
\text { (R) }\end{array}$ & Occipital bone \\
\hline NTl & $\operatorname{roup}(\Sigma=193)$ & $\begin{array}{c}4 / 6 \\
(66.67 \%)\end{array}$ & $\begin{array}{c}1 / 6 \\
(16.67 \%)\end{array}$ & $\begin{array}{c}1 / 6 \\
(16.67 \%)\end{array}$ & $\begin{array}{c}2 / 6 \\
(33.33 \%)\end{array}$ & $\begin{array}{c}3 / 6 \\
(50.00 \%)\end{array}$ & $\begin{array}{c}1 / 6 \\
(16.67 \%)\end{array}$ & $\begin{array}{c}1 / 6 \\
(16.67 \%)\end{array}$ & $\begin{array}{c}6 / 6 \\
(100.00 \%)\end{array}$ \\
\hline  & $x<25 \%$ & $\begin{array}{c}4 / 4 \\
(100.00 \%)\end{array}$ & $\begin{array}{c}1 / 1 \\
(100.00 \%)\end{array}$ & $\begin{array}{c}1 / 1 \\
(100.00 \%)\end{array}$ & $\begin{array}{c}2 / 2 \\
(100.00 \%)\end{array}$ & $\begin{array}{c}3 / 3 \\
(100.00 \%)\end{array}$ & $\begin{array}{c}1 / 1 \\
(100.00 \%)\end{array}$ & $\begin{array}{c}1 / 1 \\
(100.00 \%)\end{array}$ & $\begin{array}{c}5 / 6 \\
(83.33 \%)\end{array}$ \\
\hline  & $25 \% \leq x<50 \%$ & - & - & - & - & - & - & - & - \\
\hline  & $50 \% \leq x<75 \%$ & - & - & - & - & - & - & - & $\begin{array}{c}1 / 6 \\
(16.67 \%)\end{array}$ \\
\hline$\sqrt[x]{x}$ & $75 \% \leq x$ & - & - & - & - & - & - & - & - \\
\hline$\stackrel{\grave{\Xi}}{=}$ & Unifocal & - & - & - & - & - & $\begin{array}{c}1 / 1 \\
(100.00 \%)\end{array}$ & $\begin{array}{c}1 / 1 \\
(100.00 \%)\end{array}$ & $\begin{array}{c}1 / 6 \\
(16.67 \%)\end{array}$ \\
\hline $\bar{z} \quad \underline{\bar{z}}$ & Multifocal & $\begin{array}{c}4 / 4 \\
(100.00 \%)\end{array}$ & $\begin{array}{c}1 / 1 \\
(100.00 \%)\end{array}$ & $\begin{array}{c}1 / 1 \\
(100.00 \%)\end{array}$ & $\begin{array}{c}2 / 2 \\
(100.00 \%)\end{array}$ & $\begin{array}{c}3 / 3 \\
(100.00 \%)\end{array}$ & - & - & $\begin{array}{c}5 / 6 \\
(83.33 \%) \\
\end{array}$ \\
\hline
\end{tabular}


In the skeleton of Terry No. 522 - a 30-year-old male recorded to have died of pulmonary TB (Suppl. table 1) -, pathological bony changes that may be attributed to tuberculosis were registered in both the cranial and postcranial elements. As for the skull, multifocal GIs - described by Schultz (e.g., 1999, 2001, 2003) and Schultz \& SchmidtSchultz (2015) as pathognomonic signs of TBM, since representing pressure atrophy of the tubercles - were detected on the squamous part of the frontal bone (Fig. 49A), in the right parietal bone along the squamous suture (Fig. 49B), and on the squamous part of the left and right (Fig. 49B) temporal bones and of the occipital bone. Furthermore, non-specific vestiges of haemorrhagic and/or inflammatory meningeal reactions - namely multifocal, small, serpentine branching ABVIs accompanied by slight PAs (e.g., Schultz, 1993, 1999, 2001, 2003 ) - were observed in the left parietal and occipital bones, covering less than one-fourth (stage 1) of the inner surfaces. Moreover, shallow APDIs (very slight stage) were noted on the squamous part of the frontal bone, probably referring to eICP secondary to TBM (e.g., Schultz, 1993, 2001, 2003).

A)



B)



Figure 49: GIs A) on the squamous part of the frontal bone and $B$ ) along the squamous suture in the right parietal and temporal bones (Terry No. 522, 30-year-old, male, pulmonary TB).

Besides the endocranial alterations very likely associated with TBM, very slight PNBFs - frequently described as not specific but probable signs of pulmonary TB and/or TB pleurisy (e.g., Kelley \& Micozzi, 1984; Roberts et al., 1994; Santos \& Roberts, 2001, 2006; Maczel, 2003; Matos \& Santos, 2006; Giacon, 2008; Mariotti et al., 2015) - were noted on the visceral surface of the vertebral end of four left side $\left(4^{\text {th }}-7^{\text {th }}\right)$ and ten right side $\left(2^{\text {nd }}-11^{\text {th }}\right)$ ribs. The recorded cause of death of Terry No. 522 supports the tuberculous origin of the detected endocranial and costal changes. 
The skeletal remains of Terry No. 562 - a 17-year-old female whose morgue record states pulmonary TB as the cause of death (Suppl. table 1) - exhibited numerous pathological bony changes that probably resulted from tuberculosis. In the skull, multifocal GIs were registered on the orbital part of the frontal bone (Fig. 50A) and on the squamous part of the right temporal and occipital (Fig. 20C (p. 45), 50B) bones; whereas unifocal GIs were noted on the squamous part of the left temporal bone and on the right greater wing of the sphenoid bone. GIs were described by Schultz (e.g., 1999, 2001, 2003) and Schultz \& Schmidt-Schultz (2015) as pathognomonic vestiges of TBM. Furthermore, APDIs (slight stage) affecting the squamous part of the frontal and the left and right parietal bones were recorded, indicating eICP possibly due to hydrocephalus that may be associated with TBM (e.g., Schultz, 1993, 2001, 2003).

A)



B)



Figure 50: GIs on the right side A) of the orbital part of the frontal bone and B) of the squamous part of the occipital bone (Terry No. 562, 17-year-old, female, pulmonary TB).

With respect to the postcranial skeleton, five left side ribs $\left(2^{\text {nd }}-6^{\text {th }}\right)$ showed slight PNBFs on the visceral surface of the vertebral and sternal ends that may represent vestiges of an inflammatory response secondary to pulmonary TB and/or TB pleurisy (e.g., Kelley \& Micozzi, 1984; Roberts et al., 1994; Santos \& Roberts, 2001, 2006; Maczel, 2003; Matos \& Santos, 2006; Giacon, 2008; Mariotti et al., 2015). In the vertebral column, signs of hypervascularisation possibly referring to early-stage skeletal TB (e.g., Ménard, 1888; Baker, 1999; Maczel, 2003; Giacon, 2008; Mariotti et al., 2015) were registered on the lateral aspects of the lower thoracic (T9-12) and lumbar (L1-5) vertebral bodies. Based on the recorded cause of death of Terry No. 562, the most likely aetiology of the observed endocranial and postcranial bony changes is tuberculosis. 
Similar to the previous cases, both the cranial and postcranial remains of Terry No. 566 - a 40-year-old male who had probably died of TB (Suppl. table 1) - revealed different types of pathological bony changes that may be ascribed to tuberculosis. Regarding the skull, the orbital part of the frontal bone, the squamous part of the left temporal and occipital (Fig. 51A) bones, and the right greater wing of the sphenoid bone (Fig. 51B) exhibited GIs that were described by Schultz (e.g., 1999, 2001, 2003) and Schultz \& Schmidt-Schultz (2015) as pathognomonic vestiges of TBM. GIs covered only small areas (stage 1) of the inner surfaces of the affected bones.

A)



B)



Figure 51: Unifocal GIs A) on the left side of the squamous part of the occipital bone and B) on the right greater wing of the sphenoid bone (Terry No. 566, 40-year-old, male, probable TB).

Concerning the postcranial elements, all right side ribs showed slight PNBFs on the visceral surface of the vertebral end $\left(2^{\text {nd }}-12^{\text {th }}\right)$, body $\left(1^{\text {st }}-12^{\text {th }}\right)$, and/or sternal end $\left(3^{\text {rd }}-4^{\text {th }}\right.$, $6^{\text {th }}-7^{\text {th }}$, and $\left.9^{\text {th }}-11^{\text {th }}\right)$. PNBFs were frequently described as not specific but probable vestiges of pulmonary TB and/or TB pleurisy (e.g., Kelley \& Micozzi, 1984; Roberts et al., 1994; Santos \& Roberts, 2001, 2006; Maczel, 2003; Matos \& Santos, 2006; Giacon, 2008; Mariotti et al., 2015). The indicated cause of death of Terry No. 566 supports the tuberculous origin of the observed endocranial and costal changes.

In the skeleton of Terry No. 933R - a 40-year-old male recorded to have died of peritoneal TB (Suppl. table 1) -, numerous pathological bony changes that probably resulted from tuberculosis were noted. In the skull, the orbital (Fig. 52A) and squamous (Fig. 52B) parts of the frontal bone, the left and right parietal bones along the squamous suture, 
and the squamous part of the occipital bone (Fig. 20B (p. 45)) revealed multifocal GIs; whereas the squamous part of the left and right temporal bones, and the left and right greater wings of the sphenoid bone displayed unifocal GIs, described by Schultz (e.g., 1999, 2001, 2003) and Schultz \& Schmidt-Schultz (2015) as specific signs of TBM. Moreover, multifocal, small, serpentine branching ABVIs representing non-specific vestiges of haemorrhagic and/or inflammatory meningeal processes (e.g., Schultz, 1993, 1999, 2001, 2003) were detected on the squamous part of the left frontal bone, covering about one-third (stage 2) of the inner surface. Furthermore, shallow APDIs (very slight stage) affecting the squamous part of the frontal bone were recorded, indicating eICP possibly due to hydrocephalus that may be associated with TBM (e.g., Schultz, 1993, 2001, 2003).

A)



B)



Figure 52: Multifocal GIs on the A) orbital and B) squamous parts of the frontal bone (Terry No. 933R, 40-year-old, male, peritoneal TB).

In the postcranial skeleton, the vertebral column exhibited signs of hypervascularisation in the form of circumferential pitting on the lateral and anterior aspects of the lower cervical (C6-7) and thoracic (T1-12), as well as on the lateral aspects of the upper lumbar (L1-2) vertebral bodies. Although vertebral hypervascularisation is not a specific feature of tuberculosis, it has been described in relation to early-stage skeletal TB in a number of studies (e.g., Ménard, 1888; Baker, 1999; Maczel, 2003; Giacon, 2008; Mariotti et al., 2015). Moreover, PNBFs - probably representing vestiges of an inflammatory response secondary to pulmonary TB and/or TB pleurisy (e.g., Kelley \& Micozzi, 1984; Roberts et al., 1994; Santos \& Roberts, 2001, 2006; Maczel, 2003; Matos \& Santos, 2006; Giacon, 2008; Mariotti et al., 2015) - were noted on the visceral surface of the body of the $11^{\text {th }}$ right side rib. Based on the recorded cause of death of Terry No. 933R, the most likely aetiology of the observed endocranial and postcranial bony changes is TB. 


\subsection{Co-occurrence of endocranial alterations probably associated with tuberculosis}

\begin{tabular}{|c|c|c|c|c|c|}
\hline \multicolumn{2}{|c|}{$\Sigma=427$} & \multicolumn{2}{|c|}{ TB group } & \multicolumn{2}{|c|}{ NTB group } \\
\hline \multicolumn{2}{|c|}{$\begin{array}{l}\text { Number of specimens } \\
\text { exhibiting at least two different } \\
\text { types of endocranial alterations }\end{array}$} & \multicolumn{2}{|c|}{$\begin{array}{c}96 / 234 \\
(41.03 \%)\end{array}$} & \multicolumn{2}{|c|}{$\begin{array}{c}15 / 193 \\
(7.77 \%)\end{array}$} \\
\hline Females & Males & $\begin{array}{c}28 / 65 \\
(43.08 \%)\end{array}$ & $\begin{array}{c}68 / 169 \\
(40.24 \%)\end{array}$ & $\begin{array}{c}7 / 87 \\
(8.05 \%)\end{array}$ & $\begin{array}{c}8 / 106 \\
(7.55 \%)\end{array}$ \\
\hline
\end{tabular}

Table 9: Number of specimens exhibiting at least two different types of endocranial alterations probably related to TBM in the TB group and NTB group by sex.

From a total of 427 skeletons evaluated, $111(26.00 \%)$ showed at least two different types of the examined endocranial alterations: 96 (41.03\%) of 234 individuals recorded to have died of TB and 15 (7.77\%) of 193 specimens identified to have died of causes other than TB (Table 9); thus, there was a statistically extremely significant difference in the frequencies of the co-occurrence of endocranial lesions probably related to TBM between the TB group and NTB group $\left(\chi^{2}=59.079, \mathrm{df}=1, \mathrm{P}<0.0001\right)$. When the two groups were compared considering the sex (Table 9), the difference in the frequencies of the association of the evaluated endocranial alteration types remained extremely significant for both females $\left(\chi^{2}=23.820, \mathrm{df}=1, \mathrm{P}<0.0001\right)$ and males $\left(\chi^{2}=33.192, \mathrm{df}=1, \mathrm{P}<0.0001\right)$. However, the frequencies of the co-occurrence of the examined endocranial lesion types among females and males were very similar in both groups (Table 9); therefore, there was no statistically significant difference between the two sexes (TB group: $\chi^{2}=0.0611, \mathrm{df}=1, \mathrm{P}=0.8047$; NTB group: $\chi^{2}=0.0200, \mathrm{df}=1, \mathrm{P}=0.8876$ ).

As for the distribution of individuals exhibiting at least two different types of endocranial alterations probably related to TBM by age at death, the association of these bony changes occurred with the highest frequency among specimens under the age of 30 years $(45 / 63,71.43 \%)$ in the TB group (Fig. 53A): with $68.18 \%(15 / 22)$ among females (Fig. 53B) and with 73.17\% (30/41) among males (Fig. 53C). Nevertheless, more than onethird (40/102, 39.22\%) of individuals between 30 and 49 years of age and recorded to have died of TB were also affected by at least two different types of the evaluated endocranial lesions (females: 12/25, 48.00\%; males: 28/77, 36.36\%) (Fig. 53A-C). In the NTB group, with the exception of a 25-year-old male, specimens exhibiting association of the examined endocranial lesion types were above the age of 40 years (Fig. 54A-C). The $\chi^{2}$ testing of the frequencies of the co-occurrence of evaluated endocranial alteration types between various age groups was not assessed because of the low number of individuals in certain age groups. 
Figure 53: Demographic profile of specimens exhibiting at least two different types of endocranial alterations probably related to TBM in the TB group:

A) total sample $(96 / 234,41.03 \%)$, B) females $(28 / 65,43.08 \%)$, and C) males $(68 / 169,40.24 \%)$.

A)



$\square$ Number of specimens $(\Sigma=234)$

$\square$ Number of specimens exhibiting at least two different types of endocranial alterations $(\Sigma=96)$

B)



$\square$ Number of females $(\Sigma=65)$

$\square$ Number of females exhibiting at least two different types of endocranial alterations $(\Sigma=28)$

C)



$\square$ Number of males $(\Sigma=169)$

$\square$ Number of males exhibiting at least two different types of endocranial alterations $(\Sigma=68)$ 
Figure 54: Demographic profile of specimens exhibiting at least two different types of endocranial alterations probably related to TBM in the NTB group:

A) total sample $(15 / 193,7.77 \%)$, B) females $(7 / 87,8.05 \%)$, and C) males $(8 / 106,7.55 \%)$.

A)



$\square$ Number of specimens $(\Sigma=193)$

$\square$ Number of specimens exhibiting at least two different types of endocranial alterations $(\Sigma=15)$

B)



Age groups (years)

$\square$ Number of females $(\Sigma=87)$

$\square$ Number of females exhibiting at least two different types of endocranial alterations $(\Sigma=7)$

C)



$\square$ Number of males $(\Sigma=106)$

$\square$ Number of males exhibiting at least two different types of endocranial alterations $(\Sigma=8)$ 
Regarding the number of probable TBM-related lesion types simultaneously affecting the inner surface of the skull, from a total of 427 specimens examined, more than one-third $(162 / 427,37.94 \%)$ exhibited no signs of endocranial alterations possibly associated with TBM: 22.65\% (53/234) in the TB group (Fig. 55A) and 56.48\% (109/193) (Fig. 55B) in the NTB group. In a further one-third of individuals evaluated (154/427, 36.07\%), only one probable TBM-related lesion type occurred on the inner surface of the skull: in 85 cases with TB as the cause of death $(85 / 234,36.32 \%$ ) (Fig. 55A) and in 69 cases with NTB causes of death (69/193, 35.75\%) (Fig. 55B). Of the 96 specimens showing association of endocranial alteration types probably related to TBM in the TB group, 58 (60.42\%), 34 (35.41\%), and four $(4.17 \%)$ exhibited co-occurrence of two, three, and four types of these lesions (Fig. 55A), respectively. In the NTB group, with the exception of a 49-year-old female who was affected by three different types of probable TBM-related endocranial alterations, individuals revealing association of these bony changes displayed co-occurrence of only two types (Fig. 55B).

A)



B)



Figure 55: Distribution of specimens by number of presented probable TBM-related endocranial alteration types in the A) TB group ( $\Sigma=234)$ and B) NTB group ( $\Sigma=193)$.

Among specimens exhibiting co-occurrence of probable TBM-associated endocranial alteration types in the TB group, 74 were identified to have died of pulmonary TB (Suppl. table 1, 3). Nine additional individuals died of other types of tuberculosis, such as skeletal TB (three cases), peritoneal TB (two cases), TB meningitis (two cases), TB sinusitis (one case), and miliary TB (one case); whereas in the remaining 13 cases, the type of tuberculosis as the cause of death was not specified on the morgue record and/or death certificate (Suppl. table 1,3 ). In the NTB group, the most frequently registered NTB causes of death were cardiovascular problems (Suppl. table 2, 4) among specimens showing association of endocranial alteration types probably related to TBM. 
The skeletal remains of Terry No. 304 - a 20-year-old female whose death certificate states pulmonary TB as the cause of death (Suppl. table 1) - revealed both cranial and postcranial bony changes that probably resulted from tuberculosis. In the skull, APDIs (slight stage) were registered all over the inner surface of the skullcap and skull base, indicating eICP possibly due to hydrocephalus that may be associated with TBM (e.g., Schultz, 1993, 2001, 2003). Furthermore, non-specific vestiges of inflammatoryhaemorrhagic meningeal processes - namely multifocal, small, serpentine branching ABVIs accompanied by slight PAs (e.g., Schultz, 1993, 1999, 2001, 2003) - were detected in the APDIs in the frontal (Fig. 56) and the left and right parietal bones, covering about threefourths (stage 4) of the inner surfaces. Multifocal PAs were also noted on the squamous part of the temporal and occipital bones.



Figure 56: Multifocal ABVIs and PAs in the slight APDIs on the squamous part of the frontal bone (Terry No. 304, 20-year-old, female, pulmonary TB).

With respect to the postcranial skeleton, the vertebral column showed multiple, smooth-walled resorptive pits often connected by horizontal, superficial vascular channels on the lateral aspects of the thoracic (T4-12) vertebral bodies. The lateral aspects of the lumbar (L1-5) vertebral bodies and the ventral surface of the sacrum (S4-5) also exhibited signs of hypervascularisation, possibly referring to early-stage skeletal TB (e.g., Ménard, 1888; Baker, 1999; Maczel, 2003; Giacon, 2008; Mariotti et al., 2015). Although the observed endocranial and vertebral changes are not pathognomonic features of $\mathrm{TB}$, the cause of death of Terry No. 304 supports their tuberculous origin. 
Similar to the previous case, both the cranial and postcranial remains of Terry No. 1159 - a 26-year-old male who had died of pulmonary TB (Suppl. table 1) - displayed different types of pathological bony changes that may be ascribed to tuberculosis. Regarding the skull, GIs - described by Schultz (e.g., 1999, 2001, 2003) and Schultz \& Schmidt-Schultz (2015) as pathognomonic vestiges of TBM - were recorded on the orbital part of the frontal bone (Fig. 18C (p. 42), 57) and on the squamous part of the occipital bone, covering less than one-fourth (stage 1) of the inner surfaces. Moreover, non-specific vestiges of haemorrhagic and/or inflammatory processes of the meninges - namely multifocal, small patches of PAs (e.g., Schultz, 1993, 1999, 2001, 2003) - were detected on the squamous and orbital (Fig. 57) parts of the frontal bone, along the sagittal suture in the left and right parietal bones, and on the squamous part of the temporal and occipital bones. Furthermore, shallow APDIs (very slight stage) were registered all over the inner surface of the skullcap and skull base, indicating eICP secondary to TBM (e.g., Schultz, 1993, 2001, 2003).



Figure 57: Multifocal PAs and GIs in the APDIs on the orbital part of the frontal bone (Terry No. 1159, 26-year-old, male, pulmonary TB).

Concerning the postcranial elements, the vertebral column exhibited signs of hypervascularisation in the form of circumferential pitting on the anterior aspect of the upper thoracic (T1-4) and on the lateral aspect of the middle and lower thoracic (T5-12) and upper lumbar (L1-3) vertebral bodies, possibly referring to early-stage skeletal TB (e.g., Ménard, 1888; Baker, 1999; Maczel, 2003; Giacon, 2008; Mariotti et al., 2015). Moreover, ten left side ribs $\left(2^{\text {nd }}-11^{\text {th }}\right)$ showed very slight PNBFs on the visceral surface of the vertebral end 
$\left(2^{\text {nd }}-4^{\text {th }}\right.$ and $\left.8^{\text {th }}-9^{\text {th }}\right)$, body $\left(2^{\text {nd }}-3^{\text {rd }}, 6^{\text {th }}\right.$, and $\left.9^{\text {th }}-11^{\text {th }}\right)$, and/or sternal end $\left(2^{\text {nd }}-10^{\text {th }}\right)$ that may represent vestiges of an inflammatory response secondary to pulmonary $\mathrm{TB}$ and/or TB pleurisy (e.g., Kelley \& Micozzi, 1984; Roberts et al., 1994; Santos \& Roberts, 2001, 2006; Maczel, 2003; Matos \& Santos, 2006; Giacon, 2008; Mariotti et al., 2015). Besides the ribs, the lateral and posterior surfaces of both tibiae (predominantly the proximal portion of the shaft), the anterior surface of both humeri (particularly the distal portion of the shaft), and the supraspinatous fossa of the right scapula also revealed slight PNBFs, indicating HPO associated with pulmonary TB (e.g., Mensforth et al., 1978; Kelly et al., 1991; Winland et al., 1997; Rothschild \& Rothschild, 1998, 1999; Santos \& Roberts, 2001; Hershkovitz et al., 2002; Assis et al., 2011). Based on the recorded cause of death of Terry No. 1159, the most likely aetiology of the observed endocranial and postcranial bony changes is tuberculosis.

In the skeleton of Terry No. 1222 - a 28 -year-old female recorded to have died of pulmonary TB (Suppl. table 1) -, pathological bony changes that may be attributed to tuberculosis were registered in the cranial elements. GIs, described by Schultz (1999, 2001, 2003) and Schultz \& Schmidt-Schultz (2015) as pathognomonic features of TBM, were detected on the left greater wing of the sphenoid bone and on the squamous part of the occipital bone (Fig. 58), covering less than one-fourth (stage 1) of the inner surfaces.



Figure 58: Multifocal GIs and PAs on the squamous part of the occipital bone (Terry No. 1222, 28-year-old, female, pulmonary TB). 
Moreover, multifocal, small, serpentine branching ABVIs accompanied by slight PAs, referring to non-specific vestiges of inflammatory-haemorrhagic meningeal processes (e.g., Schultz, 1993, 1999, 2001, 2003), were noted along the sagittal sinus and around the most protruding portions of the frontal and the left and right (Fig. 59) parietal bones, covering more than two-thirds (stage 4) of the inner surfaces. The squamous part of the occipital and temporal bones, and the left and right greater wings of the sphenoid bone also revealed multifocal PAs. Shallow APDIs (very slight stage) were noted on the squamous part of the frontal bone and in the left and right parietal bones, indicating eICP secondary to TBM (e.g., Schultz, 1993, 2001, 2003). The postcranial skeleton showed no signs of bony changes probably associated with TB. The recorded cause of death of Terry No. 1222 supports the tuberculous origin of the observed endocranial alterations.



Figure 59: Multifocal ABVIs covered by PAs on the inner surface of the right parietal bone (Terry No. 1222, 28-year-old, female, pulmonary TB).

The skeletal remains of Terry No. 1322 - a 34-year-old male whose morgue record states pulmonary TB as the cause of death (Suppl. table 1) - displayed numerous pathological bony changes that probably resulted from tuberculosis. In the skull, deep APDIs (pronounced stage) were registered all over the inner surface of the skullcap and skull base, probably referring to eICP due to tuberculous involvement of the CNS (e.g., Schultz, 1993, 2001, 2003). Furthermore, non-specific vestiges of haemorrhagic and/or inflammatory 
meningeal processes - namely multifocal, small, serpentine branching ABVIs accompanied by slight PAs (e.g., Schultz, 1993, 1999, 2001, 2003) - were detected in the deep DIs and along the sagittal suture in the frontal (Fig. 19A (p. 44)) and the left and right parietal bones. Multifocal ABVIs covered by thin layers of newly formed bone (PAs) were also noted along the sagittal (Fig. 60A-B) and left transverse (Fig. 60B) sinuses in the occipital bone, and along the left sigmoid sinus (Fig. 60B) in the left temporal bone. Moreover, unifocal PAs occurred on the squamous part of the right temporal bone and on the left and right greater wings of the sphenoid bone.

A)



B)



Figure 60: Multifocal ABVIs accompanied by PAs A) along the sagittal sinus (occipital bone) and B) along the left transverse (occipital bone) and sigmoid (left temporal bone) sinuses (Terry No. 1322, 34-year-old, male, pulmonary TB).

Regarding the postcranial skeleton, three left side ribs $\left(4^{\text {th }}-6^{\text {th }}\right)$ showed very slight PNBFs on the visceral surface of the vertebral $\left(5^{\text {th }}-6^{\text {th }}\right)$ or sternal $\left(4^{\text {th }}\right)$ end that may represent signs of an inflammatory response secondary to pulmonary TB and/or TB pleurisy (e.g., Kelley \& Micozzi, 1984; Roberts et al., 1994; Santos \& Roberts, 2001, 2006; Maczel, 2003; Matos \& Santos, 2006; Giacon, 2008; Mariotti et al., 2015). In the vertebral column, multiple, smooth-walled resorptive pits often connected by horizontal, superficial vascular channels were recognised on the lateral aspects of the thoracic (T3-12) and lumbar (L1-5) vertebral bodies, indicating early-stage skeletal TB (e.g., Ménard, 1888; Baker, 1999; Maczel, 2003; Giacon, 2008; Mariotti et al., 2015). Although the observed endocranial and postcranial bony changes are not pathognomonic features of TB, the cause of death of Terry No. 1322 supports their tuberculous origin. 


\subsection{Association of endocranial and non-endocranial lesions likely related to tuberculosis}

\begin{tabular}{|c|c|c|c|c|c|}
\hline \multicolumn{2}{|c|}{$\Sigma=265$} & \multicolumn{2}{|c|}{ TB group } & \multicolumn{2}{|c|}{ NTB group } \\
\hline $\begin{array}{r}\text { Numb } \\
\text { exhibitin } \\
\text { probable } T \\
\text { and non-end }\end{array}$ & $\begin{array}{l}\text { imens } \\
\text { rrence of } \\
\text { endocranial } \\
\text { ony changes }\end{array}$ & \multicolumn{2}{|c|}{$\begin{array}{c}145 / 181 \\
(80.11 \%)\end{array}$} & \multicolumn{2}{|c|}{$\begin{array}{c}22 / 84 \\
(26.19 \%)\end{array}$} \\
\hline Females & Males & $\begin{array}{c}32 / 45 \\
(71.11 \%)\end{array}$ & $\begin{array}{c}113 / 136 \\
(83.09 \%)\end{array}$ & $\begin{array}{c}8 / 28 \\
(28.57 \%)\end{array}$ & $\begin{array}{c}14 / 56 \\
(25.00 \%)\end{array}$ \\
\hline
\end{tabular}

Table 10: Number of specimens exhibiting association of endocranial and non-endocranial bony changes probably related to tuberculosis in the TB group and NTB group by sex.

From a total of 265 skeletons showing at least one type of probable TBM-associated endocranial alterations, 167 (63.02\%) exhibited co-occurrence of possible TB-related endocranial and non-endocranial bony changes: 145 (80.11\%) of 181 individuals in the TB group and 22 (26.19\%) of 84 specimens in the NTB group (Table 10); therefore, there was a statistically extremely significant difference in the frequencies of the association of endocranial and non-endocranial lesions probably related to TB between the two groups $\left(\chi^{2}=69.280, \mathrm{df}=1, \mathrm{P}<0.0001\right)$. When the two groups were compared considering the sex (Table 10), the difference in the frequencies of the co-occurrence of possible TB-associated endocranial and non-endocranial alterations remained significant for both females $\left(\chi^{2}=10.951, \mathrm{df}=1, \mathrm{P}=0.0009\right)$ and males $\left(\chi^{2}=57.205, \mathrm{df}=1, \mathrm{P}<0.0001\right)$. However, the frequencies of the association of these bony changes in females and males were similar in both groups (Table 10); thus, there was no statistically significant difference between the two sexes (TB group: $\chi^{2}=2.339, \mathrm{df}=1, \mathrm{P}=0.1262$; NTB group: $\chi^{2}=0.00296, \mathrm{df}=1, \mathrm{P}=0.9566$ ).

Regarding the distribution of individuals showing co-occurrence of probable TBrelated endocranial and non-endocranial lesions by age at death, the association of these bony changes occurred with the highest frequency among specimens under the age of 40 years (104/119, 87.39\%): with 89.62\% (95/106) (Fig. 61A) and 69.23\% (9/13) (Fig. 62A) in the TB group and NTB group, respectively. Among individuals recorded to have died of TB, more than $90 \%$ of females under the age of 30 years $(19 / 21,90.48 \%)$ (Fig. 61B) and of males under the age of 50 years $(94 / 102,92.16 \%$ ) (Fig. 61C) showed association of possible TB-related endocranial and non-endocranial alterations; whereas among specimens identified to have died of causes other than TB, females (4/5, 80.00\%) (Fig. 62B) and males $(5 / 8,62.50 \%)$ (Fig. 62C) under the age of 40 years were most frequently affected. The $\chi^{2}$ testing of the frequencies of the co-occurrence of probable TB-associated endocranial and non-endocranial lesions between various age groups was not assessed because of the low number of individuals in certain age groups. 
Figure 61: Demographic profile of specimens exhibiting co-occurrence of endocranial and nonendocranial bony changes probably related to tuberculosis in the TB group:

A) total sample $(145 / 181,80.11 \%)$, B) females $(32 / 45,71.11 \%)$, and C) males $(113 / 136,83.09 \%)$.

A)



$\square$ Number of specimens $(\Sigma=181)$

Age groups (years)

$\square$ Number of specimens affected by both endocranial and non-endocranial bony changes $(\Sigma=145)$

B)



$\square$ Number of females $(\Sigma=45)$

Age groups (years)

$\square$ Number of females affected by both endocranial and non-endocranial bony chnages $(\Sigma=32)$

C)



$\square$ Number of males $(\Sigma=136)$

Age groups (years)

$\square$ Number of males affected by both endocranial and non-endocranial bony changes $(\Sigma=113)$ 
Figure 62: Demographic profile of specimens exhibiting co-occurrence of endocranial and nonendocranial bony changes probably related to tuberculosis in the NTB group:

A) total sample $(22 / 84,26.19 \%)$, B) females $(8 / 28,28.57 \%)$, and C) males $(14 / 56,25.00 \%)$.

A)



Age groups (years)

$\square$ Number of specimens $(\Sigma=84)$

$\square$ Number of specimens affected by both endocranial and non-endocranial bony changes $(\Sigma=22)$

B)

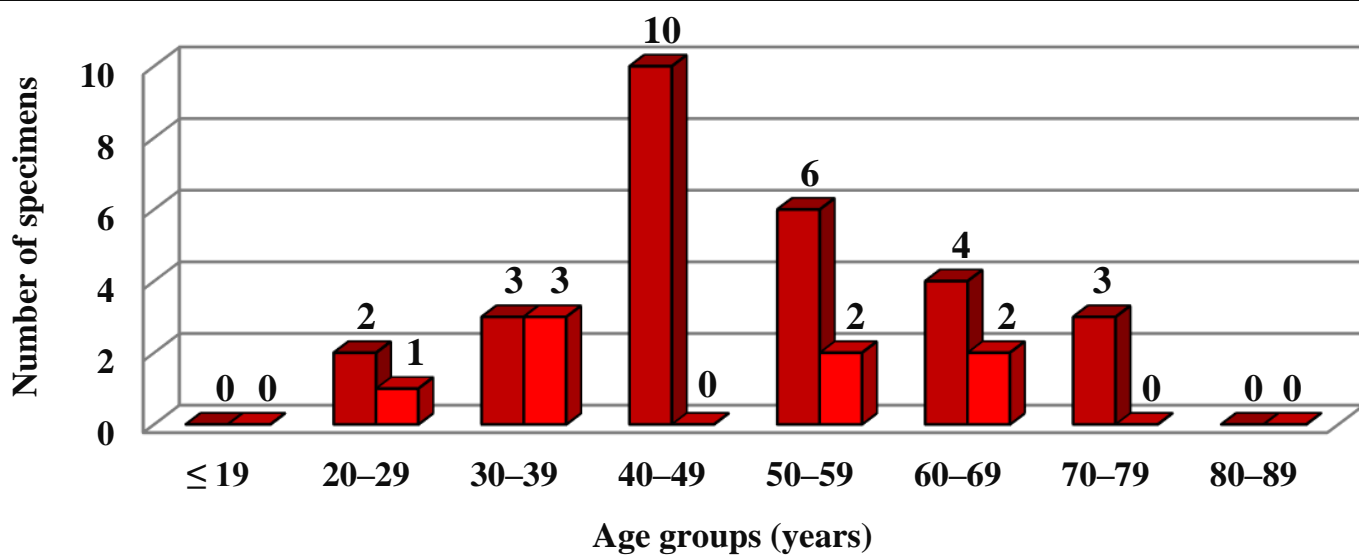

$\square$ Number of females $(\Sigma=28)$

$\square$ Number of females affected by both endocranial and non-endocranial bony chnages $(\Sigma=8)$

C)



Number of males $(\Sigma=56)$

$\square$ Number of males affected by both endocranial and non-endocranial bony changes $(\Sigma=14)$ 
As for the number of probable TB-related non-endocranial lesion types simultaneously affecting the skeleton, from a total of 265 specimens showing at least one type of possible TBM-associated endocranial alterations, more than one-third $(98 / 265,36.98 \%)$ exhibited no signs of non-endocranial bony changes: $19.89 \%$ (36/181) in the TB group (Fig. 63A) and $73.81 \%$ (62/84) (Fig. 63B) in the NTB group. In a further one-fourth of individuals evaluated $(61 / 265,23.02 \%)$, only one probable TB-associated non-endocranial lesion type occurred in the skeleton: in 47 cases with TB as the cause of death (47/181, 25.97\%) (Fig. 63A) and in 14 cases with NTB causes of death $(14 / 84,16.67 \%)$ (Fig. 63B). Of the 106 specimens showing association of non-endocranial alteration types probably related to TB (106/265, $40.00 \%)$, about two-thirds $(70 / 106,66.04 \%)$ exhibited co-occurrence of two types of these bony changes: $67.35 \%(66 / 98)$ in the TB group (Fig. 63A) and $50.00 \%(4 / 8)$ in the NTB group (Fig. 63B). The association of more than two possible TB-related non-endocranial lesions was detected in about one-third $(36 / 106,33.96 \%)$ of individuals exhibiting cooccurrence of these alterations: in 32 cases $(32 / 98,32.65 \%)$ with TB as the cause of death (Fig. 63A) and in four cases $(4 / 8,50.00 \%)$ with NTB causes of death (Fig. 63B). Nevertheless, the number of probable TB-related non-endocranial bony changes concurrently affecting the skeleton varied from two to seven in the TB group and from two to four in the NTB group (Fig. 63A-B).

A)



B)



Figure 63: Distribution of specimens with at least one type of probable TBM-related endocranial alterations by number of presented possible TB-associated non-endocranial alteration types in the A) TB group $(\Sigma=181)$ and B) NTB group $(\Sigma=84)$. 
Concerning the frequencies of different types of possible TB-associated nonendocranial bony changes affecting specimens with at least one type of probable TBMrelated endocranial alterations in the TB group, PNBFs on the visceral surface of ribs $(111 / 181,61.33 \%)$ and signs of hypervascularisation on the anterior and/or lateral aspects of vertebral bodies $(102 / 181,56.35 \%)$ were the most frequently detected lesions; whereas TB involvement of the extra-spinal bones (i.e., TB osteomyelitis) affecting the ribs, hip bones, cranial bones or sternum $(13 / 181,7.18 \%)$, as well as of the extra-spinal joints (i.e., TB arthritis) - such as of the sacroiliac joint and/or pubic symphysis $(11 / 181,6.08 \%)-$, was registered in only a few cases (Fig. 64A). In the NTB group, circumferential, multiple, smooth-walled resorptive lesions often connected by horizontal vascular impressions on the anterior and lateral aspects of the vertebral bodies $(13 / 84,15.48 \%)$ represented the most frequently observed non-endocranial bony changes possibly associated with TB (Fig. 64B).

A)



B)



Figure 64: Number of specimens with at least one type of probable TBM-related endocranial alterations and showing different types of possible TB-associated non-endocranial bony changes in the A) TB group $(\Sigma=181)$ and B) NTB group $(\Sigma=84)$.

Among individuals with association of probable TB-related endocranial and nonendocranial bony changes in the TB group, the type of tuberculosis as the cause of death was not specified on the morgue record and/or death certificate in 21 cases (Suppl. table 1, 5). In 114 cases, pulmonary TB was registered as the cause of death (Suppl. table 1, 5); whereas the remaining ten individuals have died of skeletal TB (three cases), peritoneal TB (three cases), TB meningitis (two cases), TB sinusitis (one case) or miliary TB (one case) (Suppl. table 1, 5). Among specimens with co-occurrence of possible TB-associated endocranial 
and non-endocranial bony changes in the NTB group, the most frequently registered NTB causes of death were cardiovascular problems (Suppl. table 2, 6).

In the skeleton of Terry No. 468 - a 23-year-old male recorded to have died of pulmonary and spinal TB (Suppl. table 1) -, pathological bony changes that may be attributed to tuberculosis were registered both in the cranial and postcranial elements (Spekker et al., 2018). In the skull, APDIs (very slight stage) were registered all over the inner surface of the skullcap and skull base, indicating eICP very likely due to hydrocephalus that may be associated with TBM (e.g., Schultz, 1993, 2001, 2003).

Concerning the postcranial bone remains, PNBFs - frequently described as not pathognomonic but probable signs of pulmonary TB and/or TB pleurisy (e.g., Kelley \& Micozzi, 1984; Roberts et al., 1994; Santos \& Roberts, 2001, 2006; Maczel, 2003; Matos \& Santos, 2006; Giacon, 2008; Mariotti et al., 2015) - occurred on the vertebral $\left(11^{\text {th }}-12^{\text {th }}\right)$ (Fig. 65) and/or sternal $\left(12^{\text {th }}\right)$ ends of two left side ribs $\left(11^{\text {th }}-12^{\text {th }}\right)$, exclusively affecting the visceral surfaces.



Figure 65: Slight PNBFs on the visceral surface of the vertebral end of the $11^{\text {th }}$ left rib (Terry No. 468, 23-year-old, male, pulmonary and spinal TB) (Spekker et al., 2018).

Besides the above-mentioned rib lesions, vertebral alterations very likely associated with the anterior subligamentous form of tuberculous spondylitis (Sorrel \& Sorrel-Dejerine, 1932; Aufderheide \& Rodríguez-Martín, 1998; Palmer, 2002; Ortner, 2003; Kumar, 2005; Spiegel et al., 2005; Agrawal et al., 2010; Garg \& Somvanshi, 2011; Rivas-Garcia et al., 2013) were observed in the thoracic (T6-12) and lumbar (L1-5) regions. The anterior and occasionally the lateral aspects of the middle and lower thoracic (T6-12) vertebral bodies revealed slight erosion and reactive new bone formations exclusively affecting the cortical bone layers. In addition to the aforementioned superficial changes, the lower thoracic 
(T8-12) vertebral bodies exhibited small, shallow erosive lesions, particularly localised on the anterior and right lateral aspects (Fig. 66A-B). Similar to the thoracic region of the spine, the anterior and lateral aspects of the lumbar (L1-4) vertebral bodies (Fig. 67A-C) displayed severe destructive remodelling of the cortical bone layers, occasionally extending towards the posterior vertebral elements. Moreover, multiple, tunnel- or groove-like erosive lesions involving the anterior, lateral, superior, and/or inferior surfaces, as well as remarkable new bone formations and bony bridges interconnecting the anterior aspects of the adjacent lumbar vertebral bodies (L1-4) (Fig. 67A-C), were detected. The L5 body was also affected by the pathological process: a shallow erosive lesion in the middle of the postero-superior surface, signs of slight hypervascularisation in the form of circumferential pitting on the anterior and lateral aspects, and reactive new bone formations extending towards the posterior vertebral elements on both lateral aspects were noted.

A)



B)



Figure 66: Slight cortical erosion accompanied by PNBFs on the anterior and lateral aspects of the T6-10 vertebral bodies: $A$ ) right antero-lateral view and B) left antero-lateral view (Terry No. 468, 23-year-old, male, pulmonary and spinal TB) (Spekker et al., 2018). 
Figure 67: Deep, spherical, tunnel- or groove-like erosive lesions and bony bridges interconnecting the adjacent vertebral bodies in the lumbar region (L1-4): A) right antero-lateral view, B) anterior view, and C) left antero-lateral view (Terry No. 468, 23-year-old, male, pulmonary and spinal TB) (Spekker et al., 2018).

A)



B)

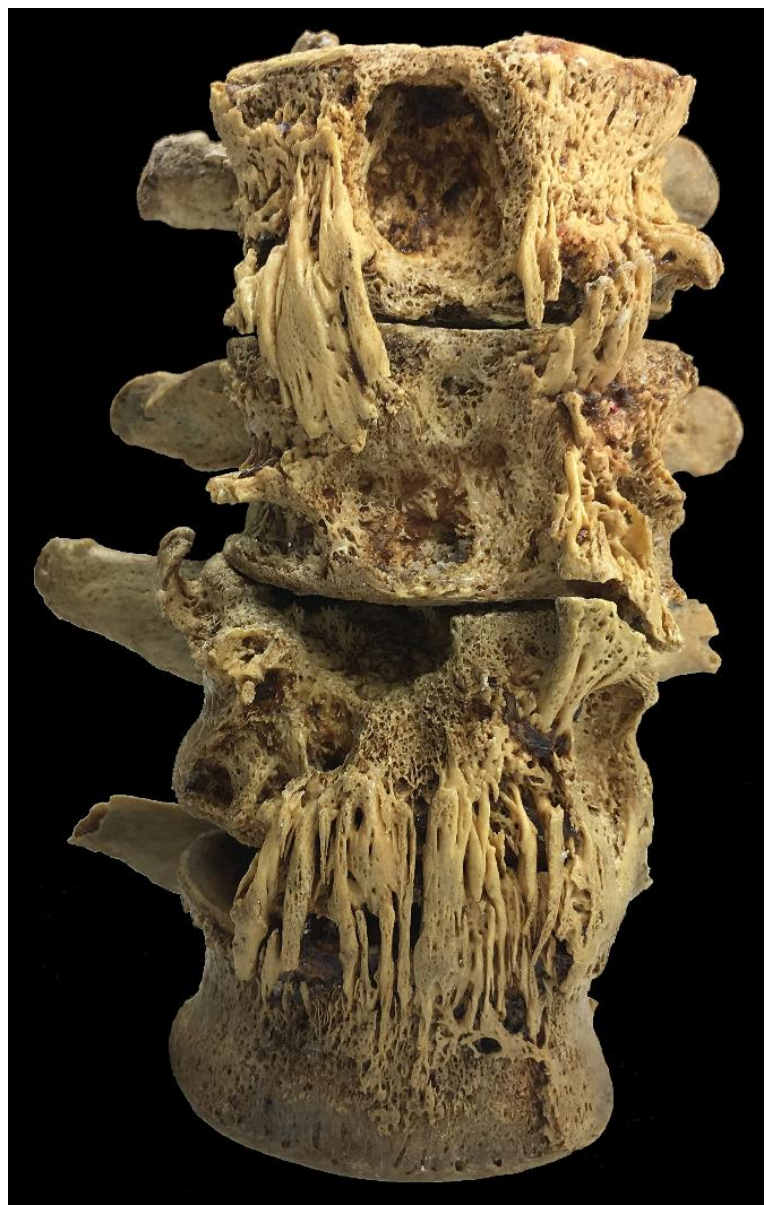

C)




Probably in response to an overlying extra-vertebral cold abscess that is a common complication in tuberculous spondylitis (Aufderheide \& Rodríguez-Martín, 1998; Ortner, 2003; Pálfi et al., 2012), an erosive lesion accompanied by PNBFs was detected on the anterior surface of the left and right (Fig. 68A) hip bones, adjacent to the medial and middle parts of the iliac crest. Furthermore, the iliac and sacral articular surfaces of the left and right (Fig. 68A) sacroiliac joints showed a slightly porous appearance, possibly referring to bilateral TB sacroiliitis. Nevertheless, tuberculous involvement of the sacroiliac joint is rather uncommon, affecting approximately 3 to $10 \%$ of all cases with osteoarticular TB and usually results from direct extension of a spinal TB focus (Aufderheide \& Rodríguez-Martín, 1998; Ortner, 2003; Gupta et al., 2005; Papagelopoulos et al., 2005). The two pubic symphyses were also affected by the pathological process: both the left (Fig. 68B) and right adjoining surfaces exhibited severe destruction that is suggestive of TB symphysitis, representing an unusual site of the disease (Ortner, 2003; Lal et al., 2013; Meena \& Gangary, 2015).

A)



B)



Figure 68: A) shallow, roundish erosive lesion accompanied by PNBFs on the anterior surface of the right hip bone, adjacent to the iliac crest (white circle), as well as slight erosive lesions on the iliac articular surface of the right sacroiliac joint (white arrow), and B) severe destruction of the adjoining surface of the left pubic symphysis (Terry No. 468, 23-year-old, male, pulmonary and spinal TB)

(Spekker et al., 2018). 
Similar to the previous case, both the cranial and postcranial remains of Terry No. 902 - an approximately 36-year-old male who had died of pulmonary TB (Suppl. table 1) - showed different types of pathological bony changes that may be ascribed to tuberculosis (Spekker et al., 2018). Concerning the skull, multifocal, small, serpentine branching ABVIs - described by Schultz (e.g., Schultz, 1993, 1999, 2001, 2003) as non-specific vestiges of haemorrhagic and/or inflammatory processes of the meninges - were registered on the endocranial surface of the frontal (Fig. 69A) and the left and right (Fig. 69B) parietal bones. Although ABVIs are not pathognomonic features of tuberculosis, they may result from TBM (e.g., Schultz, 1993, 1999, 2001, 2003; Hershkovitz et al., 2002; Lewis, 2004).

A)



B)



Figure 69: Multifocal, small, serpentine branching ABVIs on the endocranial surface A) of the left side of the squamous part of the frontal bone and $B$ ) of the right parietal bone (Terry No. 902, 36-year-old, male, pulmonary TB) (Spekker et al., 2018).

Regarding the postcranial elements, four non-contiguous foci of probable tuberculous spondylitis were identified: the left atlanto-occipital joint, the middle thoracic region (T47), the T12 vertebra, and the lower lumbar region (L4-5). The articular surfaces of the left atlanto-occipital joint (Fig. 70A-B) - especially the one located on the $\mathrm{C} 1$ - showed a rough porous appearance and severe destruction. Moreover, reactive new bone formations were registered posteriorly to the left condyle of the occipital bone, adjacent to the foramen magnum (Fig. 70A). The articular surfaces of the left facet joint between the T4-5 vertebrae (Fig. 70C-D) were also affected by the pathological process, exhibiting a rough porous appearance and slight destruction. The above-mentioned articular lesions may represent the 
articular form of tuberculous spondylitis (Ortner, 2003; Garg \& Somvanshi, 2011; Fuentes Ferrer et al., 2012; Qureshi et al., 2013; Mansukhani et al., 2014).

A)

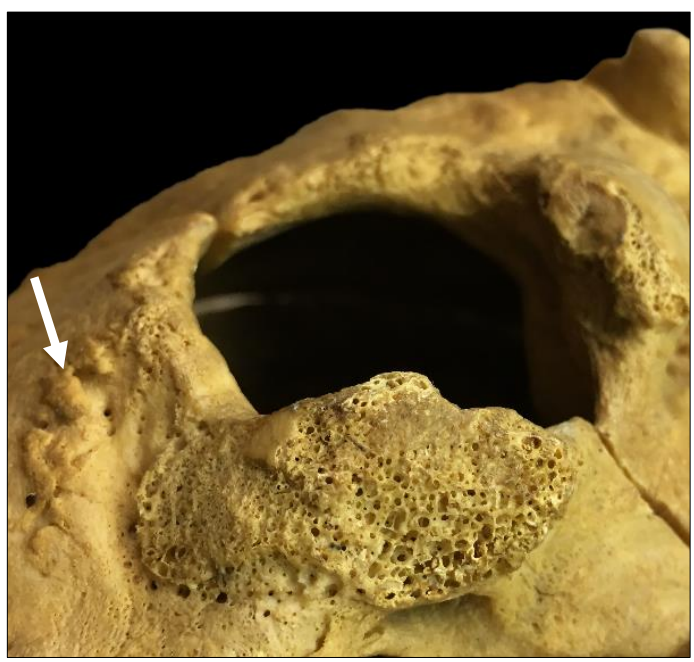

C)

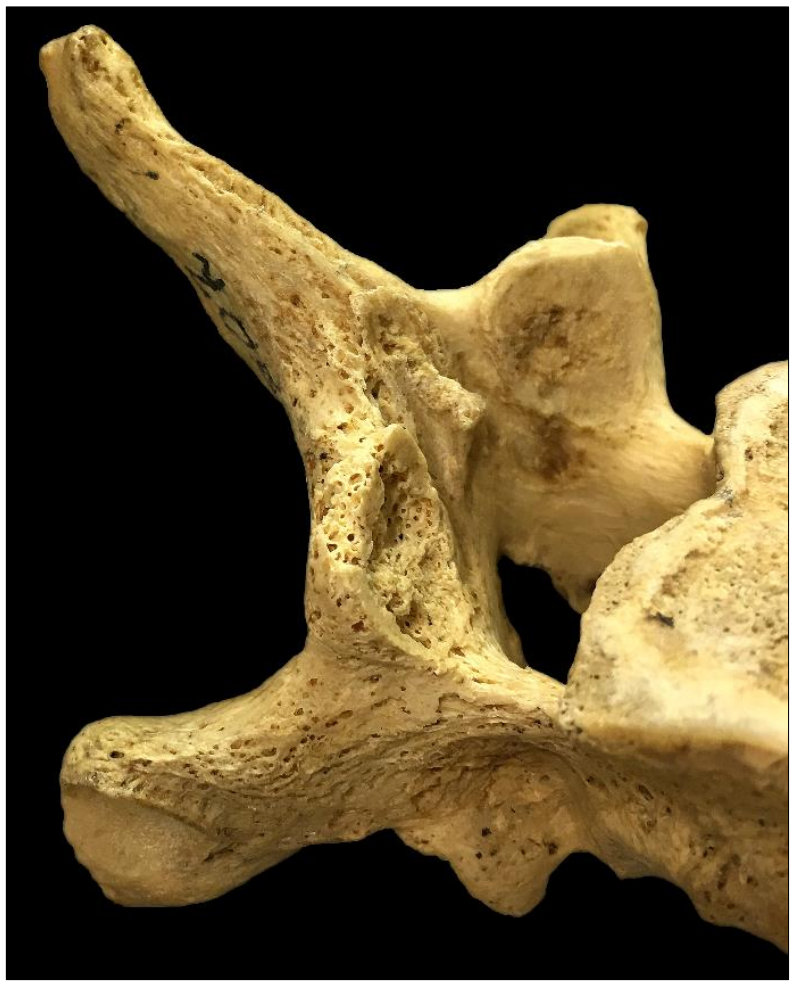

B)

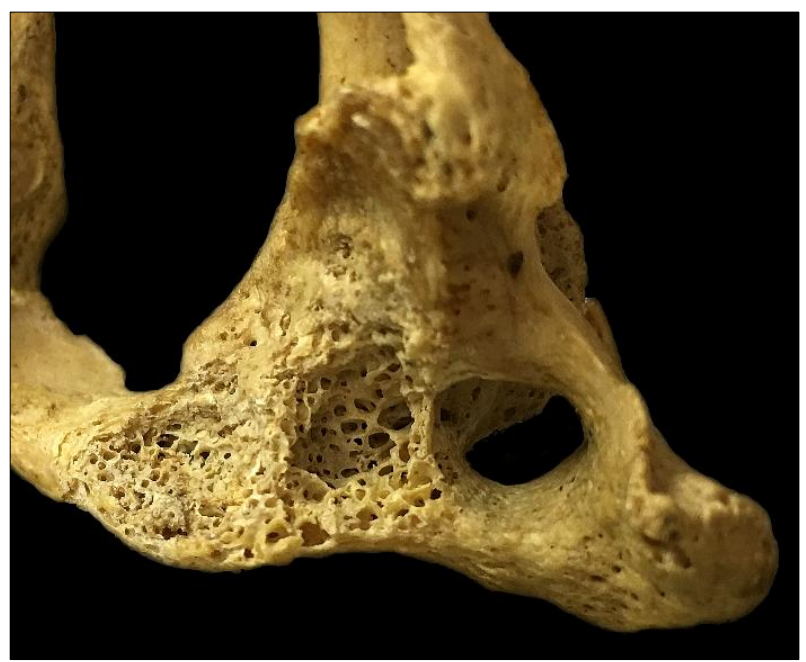

D)

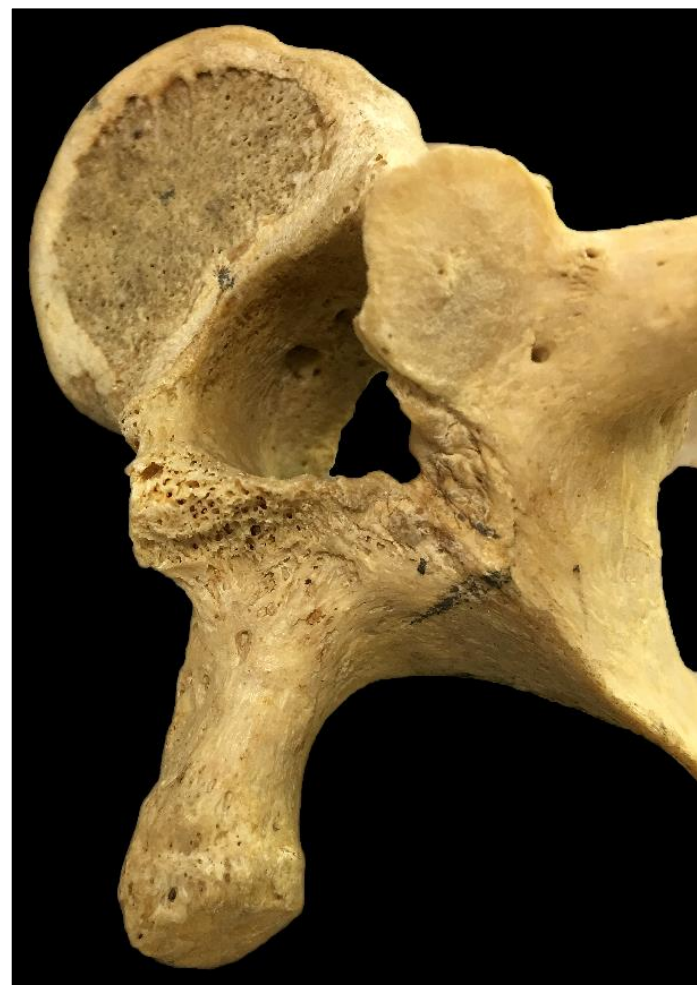

Figure 70: Erosion of the adjoining articular surfaces in the left atlanto-occipital joint: A) occipital bone (with new bone formations adjacent to the foramen magnum - white arrow) and B) C1, as well as in the left facet joint between the T4-5 vertebrae: C) $\mathrm{T} 4$ and D) $\mathrm{T} 5$ (Terry No. 902, 36-year-old, male, pulmonary TB) (Spekker et al., 2018).

Furthermore, the anterior aspect of the T5-6 bodies (Fig. 71A) revealed slight remodelling of the cortical bone accompanied by small, abnormal, worm-like blood vessel impressions. Erosive lesions involving the left lamina and the adjoining part of the spinous 
process of T4 (Fig. 71B), as well as the right lateral aspect of the T7 body, were also recorded. On the anterior aspect of the T12 and L4-5 vertebral bodies, slight remodelling of the cortical bone was detected. Moreover, the right lateral aspect of the T12 body (Fig. 71C) and the left lateral aspect of the L5 body (Fig. 71D) revealed erosive lesions accompanied by reactive new bone formations. The aforementioned superficial vertebral changes may indicate the presence of extra-vertebral TB abscesses that may extend from the affected joints into the posterior vertebral elements and/or descend down beneath the anterior longitudinal ligament into lower areas of the spine, probably representing the anterior subligamentous form of tuberculous spondylitis (Sorrel \& Sorrel-Dejerine, 1932; Aufderheide \& Rodríguez-Martín, 1998; Palmer, 2002; Ortner, 2003; Kumar, 2005; Spiegel et al., 2005; Agrawal et al., 2010; Garg \& Somvanshi, 2011; Rivas-Garcia et al., 2013).

A)

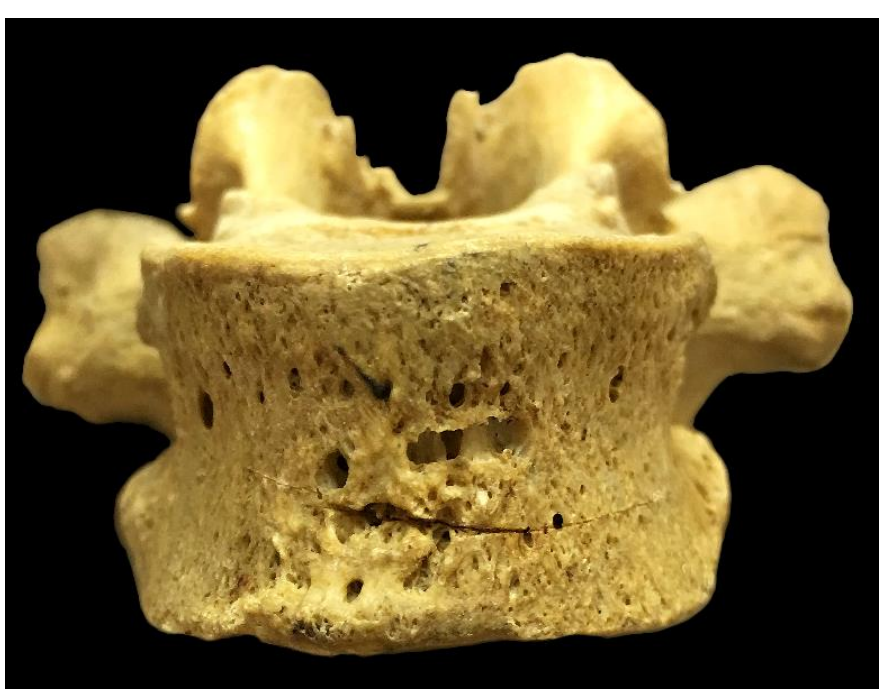

B)



C)

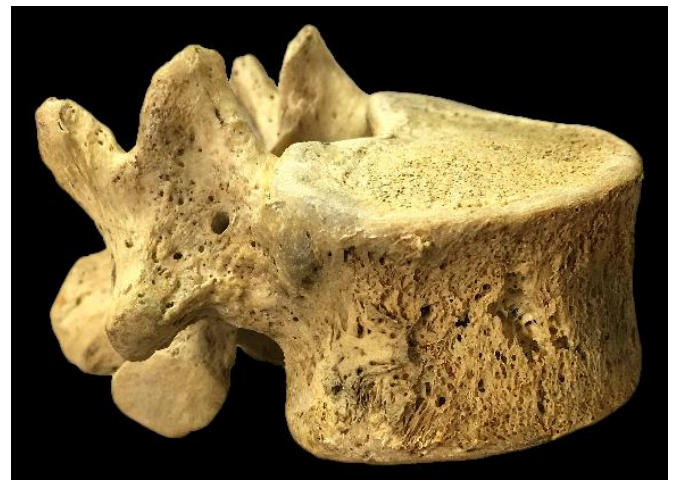

D)



Figure 71: Superficial vertebral changes: A) slight cortical remodelling and small, horizontal abnormal blood vessel impressions on the anterior aspect of T6; and erosive lesions accompanied by reactive new bone formations on the $B$ ) left lamina and spinous process of $\mathrm{T} 4, \mathrm{C}$ ) right lateral aspect of $\mathrm{T12}$, and D) left lateral aspect of L5 (Terry No. 902, 36-year-old, male, pulmonary TB) (Spekker et al., 2018). 
Moreover, signs of hypervascularisation were noted in the form of circumferential pitting on the lateral aspects of the lower thoracic (T10-12) and lumbar (L1-5) vertebral bodies. Although vertebral hypervascularisation is not a specific feature of tuberculosis, it has been described in relation to early-stage skeletal TB in a number of studies (e.g., Ménard, 1888; Baker, 1999; Maczel, 2003; Giacon, 2008; Mariotti et al., 2015).

Besides the vertebral changes, multiple perforating lytic lesions, as well as remodelling and destruction of the cortical surfaces on both the external (Fig. 72A) and internal (Fig. 72B) surfaces of the body of the sternum were detected, probably indicating TB osteomyelitis of the sternum. However, TB involvement of the sternum is fairly uncommon, accounting for less than $2 \%$ of all cases with extra-spinal TB osteomyelitis (Aufderheide \& Rodríguez-Martín, 1998; Ortner, 2003; Vasa et al., 2009; Sachdeva et al., 2013).

A)

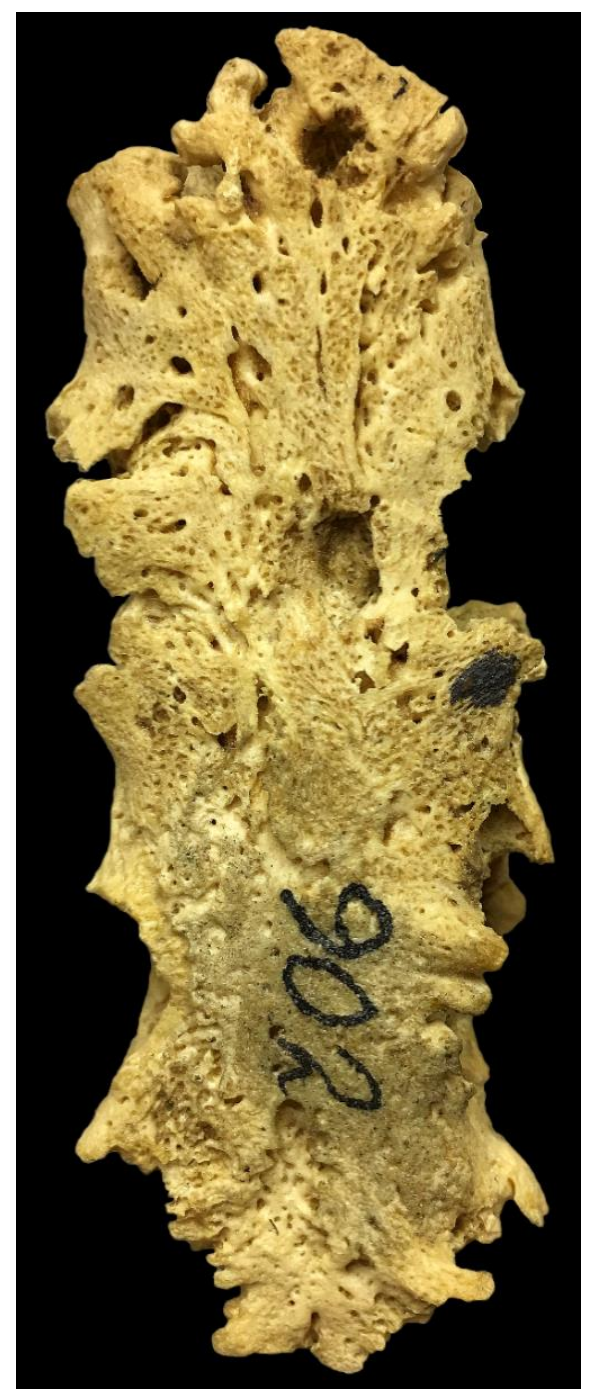

B)



Figure 72: Severe destruction and remodelling of the A) external and B) internal surfaces of the body of the sternum, with multiple perforating lytic lesions (Terry No. 902, 36-year-old, male, pulmonary TB) (Spekker et al., 2018). 
In addition, very slight PNBFs were recognised on the vertebral end of six right side $\left(3^{\text {rd }}-8^{\text {th }}\right)$ and two left side $\left(4^{\text {th }}-5^{\text {th }}\right)$ ribs, exclusively affecting the visceral surfaces. Furthermore, solitary erosive lesions were observed on the body (left $3^{\text {rd }}$ and right $4^{\text {th }}$ ) and/or sternal end (left $4^{\text {th }}-7^{\text {th }}$ ) of six ribs (Fig. 73). According to the results of previous palaeopathological studies performed on human bone remains from documented skeletal collections (e.g., Kelley \& Micozzi, 1984; Roberts et al., 1994; Santos \& Roberts, 2001, 2006; Maczel, 2003; Matos \& Santos, 2006; Giacon, 2008; Mariotti et al., 2015), the abovementioned rib lesions may represent signs of an inflammatory response secondary to pulmonary TB and/or TB pleurisy.



Figure 73: Small erosive lesion in the costal groove of the $4^{\text {th }}$ left side rib (Terry No. 902, 36-year-old, male, pulmonary TB) (Spekker et al., 2018).

All surfaces of both tibiae (predominantly the middle and distal portions of the shaft) (Fig. 74) and fibulae (all along the shaft), and the palmar surfaces of four left side $\left(2^{\text {nd }}-5^{\text {th }}\right)$ and four right side $\left(1^{\text {st }}-4^{\text {th }}\right)$ metacarpals (mainly the middle and distal portions of the shaft) also exhibited slight PNBFs, presumably referring to HPO associated with pulmonary TB (e.g., Kelly et al., 1991; Winland et al., 1997; Rothschild \& Rothschild, 1998, 1999; Santos \& Roberts, 2001; Hershkovitz et al., 2002; Assis et al., 2011).



Figure 74: Slight PNBFs on the medial surface of the mid-shaft of the left tibia (Terry No. 902, 36-year-old, male, pulmonary TB) (Spekker et al., 2018). 
Similar to the previous case, signs of an overlying TB cold abscess (Aufderheide \& Rodríguez-Martín, 1998; Ortner, 2003; Pálfi et al., 2012) were noted in the form of small erosive lesions accompanied by PNBFs on the anterior surface of the right hip bone, adjacent to the middle part of the iliac crest. Moreover, both the iliac (Fig. 75A) and sacral (Fig. 75B) articular surfaces of the left sacroiliac joint showed a rough porous appearance, very likely suggesting unilateral TB sacroiliitis (Aufderheide \& Rodríguez-Martín, 1998; Ortner, 2003; Gupta et al., 2005; Papagelopoulos et al., 2005).

A)



B)



Figure 75: Slight erosion on the A) iliac and B) sacral articular surfaces of the left sacroiliac joint (Terry No. 902, 36-year-old, male, pulmonary TB) (Spekker et al., 2018). 
5 DISCUSSION \& CONCLUSIONS 
Tuberculosis is one of the oldest known infectious diseases that has been plaguing mankind for thousands of years (Galagan, 2014). The palaeopathological research of TB essentially based upon the macromorphological diagnosis of the disease in ancient human bone remains may provide invaluable data on the different manifestations of tuberculosis, as well as on the effects of TB upon human mortality and morbidity around the world throughout prehistoric and historic times (Maczel, 2003; Santos \& Roberts, 2006; Pálfi et al., 2015).

Using modern medical knowledge, palaeopathologists attempt to establish a retrospective diagnosis of prehistoric and historic cases with tuberculosis by macroscopically identifying pathological conditions (e.g., spinal TB and TB arthritis of the large, weight-bearing joints) in skeletons of people lived in the past that may be related to the disease. However, utilisation of modern diagnostic criteria for tuberculosis in the palaeopathological practice may not be appropriate, since on the one hand, probable TBrelated bony changes observed in recent cases may differ from those of detectable in ancient human bone remains, due in part to the introduction of antibiotics in the treatment of tuberculosis. On the other hand, in modern medical TB cases, bony changes cannot be surveyed with macromorphological methods but with medical imaging techniques (e.g., Xray radiography, computed tomography, and magnetic resonance imaging) only; nevertheless, subtle bony alterations may be impossible to be visualised by the latter ones. Therefore, they are not relevant to the diagnosis of tuberculosis in recent cases and are not described as diagnostic criteria for the disease by physicians in the modern medical literature, even if they can be potentially important elements of TB identification for palaeopathologists. Furthermore, the assessment of TB prevalence in past human populations has traditionally relied upon the palaeopathological diagnosis of spinal TB and/or TB arthritis of the large, weight-bearing joints only. Since osteoarticular TB occurs in less than $2 \%$ of all patients with active tuberculosis and according to estimates, accounted for approximately 3 to $5 \%$ of all TB cases in prehistoric and historic times, it is difficult to assess the true prevalence of the disease in human osteoarchaeological material from the preantibiotic era based only on the above-mentioned diagnostic criteria (Kelley \& Micozzi, 1984; Roberts et al., 1994; Santos \& Roberts, 2001, 2006; Maczel, 2003; Ortner, 2003; Roberts \& Buikstra, 2003; Matos \& Santos, 2006; Pálfi et al., 2012; Mariotti et al., 2015).

In order to contribute to facilitating the establishment of a more reliable and accurate palaeopathological diagnosis of TB and the assessment of a more relevant disease prevalence in past human populations, a number of palaeopathological and palaeomicrobiological 
studies (e.g., Ménard, 1888; Kelley \& Micozzi, 1984; Schultz, 1993, 1999, 2001, 2003; Roberts et al., 1994; Templin \& Schultz, 1994; Teschler-Nicola et al., 1994, 2015; Jankauskas \& Schultz, 1995; Winland et al., 1997; Rothschild \& Rothschild, 1998, 1999; Baker, 1999; Jankauskas, 1999; Haas et al., 2000; Santos \& Roberts, 2001, 2006; Hershkovitz et al., 2002; Maczel, 2003; Matos \& Santos, 2006; Giacon, 2008; Pálfi et al., 2012; Mariotti et al., 2015; Masson et al., 2015; Molnár et al., 2015; Schultz \& SchmidtSchultz, 2015) were performed on osteoarchaeological series and documented skeletal collections since the late $20^{\text {th }}$ century that have revealed a positive correlation between different types of tuberculosis (e.g., skeletal TB, pulmonary TB and/or TB pleurisy, and TBM) and subtle bony alterations, including vertebral hypervascularisation, PNBFs on the visceral surface of ribs, and endocranial alterations (i.e., abnormally pronounced digital impressions; periosteal appositions; abnormal blood vessel impressions, including serpens endocrania symmetrica; and granular impressions). However, the diagnostic value of different endocranial alteration types possibly associated with TBM has more recently been questioned (e.g., Lewis, 2004; Roberts et al., 2009; Janovic et al., 2015), as their precise aetiology is still a matter of controversy, and additionally, similar or even the same morphological features can be found in non-TB-related cases, such as in non-specific inflammatory (e.g., non-specific meningitis) and haemorrhagic (e.g., epidural haematoma) processes.

Detailed analysis of well-documented collections of pre-antibiotic era skeletons of known cause of death (e.g., Hamann-Todd Human Osteological Collection, Robert J. Terry Anatomical Skeletal Collection, and Coimbra Identified Skeletal Collection) can serve as a unique and important basis for determining the appropriate palaeopathological diagnostic criteria for TB in past human populations, since bone remains of specimens identified to have died of tuberculosis and not treated with antibiotics may exhibit similar TB-related bony changes to those of observable in skeletons of people lived in the past; in contrast to recent cases with tuberculosis, they can be surveyed not only with medical imaging techniques but also directly with macromorphological methods; and even subtle bony changes can be recognised in them (Santos \& Roberts, 2001, 2006; Hunt \& Albanese, 2005; Matos \& Santos, 2006; Mariotti et al., 2015). In the last three decades, the Terry Collection has been used to define and refine palaeopathological diagnostic criteria for tuberculosis in several studies (e.g., Roberts et al., 1994; Winland et al., 1997; Rothschild \& Rothschild, 1998, 1999; Pálfi et al., 2012); nevertheless, the possible TBM-associated endocranial alteration types were beyond the scope of the aforementioned research projects. 
The main aim of the current $\mathrm{PhD}$ dissertation was to expand knowledge and understanding about the development of the four probable TBM-related endocranial alteration types (i.e., APDIs, PAs, ABVIs, and GIs) and to improve their palaeopathological interpretation, as well as to contribute to strengthening their diagnostic value in the identification of tuberculosis in human osteoarchaeological material. Therefore, review of the modern medical and palaeopathological literature regarding tuberculosis was conducted, with special attention to bony changes likely associated with TB. Moreover, for the first time, a detailed investigation focusing on the macromorphological characteristics and frequencies of the aforementioned endocranial alterations, as well as of their co-occurrence with each other and with non-endocranial bony changes probably related to TB, was performed on all individuals $(\mathrm{N}=302)$ recorded to have died of different types of tuberculosis (e.g., pulmonary TB, miliary TB, peritoneal TB, and skeletal TB) in the Terry Collection, and on a control group consisting of randomly selected specimens $(\mathrm{N}=302)$ from the remaining skeletons of the Terry Collection, identified to have died of causes other than TB (e.g., other infectious diseases, cardiovascular problems, cancer, and external causes, such as suicide and homicide). Nonetheless, it must be noted that the disease registered as the cause of death on the morgue record and/or death certificate of the 604 individuals selected for the above-mentioned research project from the Terry Collection may not have been the only medical condition present in the specimens; thus, even if another medical condition was registered as the cause of death on the morgue record and/or death certificate of individuals recorded to have died of causes other than TB from the Terry Collection, they could still have suffered from tuberculosis at death (Roberts et al., 1994; Santos \& Roberts, 2001).

From the 604 selected skeletons surveyed in the Terry Collection, 177 were excluded from the examination considering the four endocranial alteration types possibly associated with TBM: the skullcap was missing in two cases and the skull was not sectioned in a further 173 cases; therefore, precluding the accurate observation of the inner surface of the skull; whereas the age at death was uncertain in two additional cases; thus, compromising the statistical analysis of data. The remaining 427 individuals with skulls sectioned in the transverse plane and occasionally also in the mid-sagittal plane were divided into two main groups on the basis of their causes of death: one composed of 234 specimens with TB as the cause of death (TB group) and the other consisting of 193 individuals with NTB causes of death (NTB group). 
The present $\mathrm{PhD}$ dissertation focused on the macromorphological and statistical results of the aforementioned research project, with objectives of the dissertation being the following:

1) To macroscopically evaluate the 427 selected skeletons with sectioned skulls from the Terry Collection for the presence of the four types of probable TBM-related endocranial alterations, as well as for their co-occurrence with each other and with non-endocranial bony changes possibly associated with tuberculosis;

2) To compare the frequencies of the four types of probable TBM-related endocranial alterations, as well as of their co-occurrence with each other and with non-endocranial bony changes possibly associated with tuberculosis, between the 234 individuals recorded to have died of TB (TB group) and the 193 specimens identified to have died of causes other than TB (NTB group), considering sex and age at death of individuals;

3) To macromorphologically characterise the four probable TBM-related endocranial alteration types detected in the 427 selected skeletons with sectioned skulls from the Terry Collection, regarding the prominence (APDIs), as well as the localisation, extent, and number (PAs, ABVIs, and GIs) of lesions on the affected cranial bone(s);

4) To provide example cases showing the most important macromorphological characteristics of the four types of possible TBM-associated endocranial alterations; and

5) To evaluate the diagnostic value of the four probable TBM-related endocranial alteration types examined in the 427 selected skeletons with sectioned skulls from the Terry Collection.

The subsequent section of the current $\mathrm{PhD}$ dissertation provides detailed discussion of the macromorphological and statistical results of the research project conducted on the 427 selected skeletons with sectioned skulls from the Terry Collection and draws conclusions deriving from the findings in order of the above-mentioned objectives.

1) During the macromorphological evaluation of the 427 selected skeletons with sectioned skulls from the Terry Collection, 50.59\% (216/427), 15.69\% (67/427), 14.52\% (62/427), and $17.33 \%$ (74/427) of the surveyed individuals exhibited APDIs, PAs, ABVIs, and GIs, respectively. Therefore, APDIs represented the most frequently detected type of possible TBM-associated endocranial alterations; whereas PAs, ABVIs, and GIs occurred in similar proportions of the examined specimens. Furthermore, of the 427 skeletons, more than 
one-third (162/427, 37.94\%) displayed no signs of endocranial alterations probably related to TBM. In a further one-third $(154 / 427,36.07 \%)$ of individuals, only one possible TBMassociated endocranial alteration type was registered on the inner surface of the skull; whereas the remaining less than one-third $(111 / 427,25.99 \%)$ of specimens showed cooccurrence of at least two different types of endocranial alterations likely related to TBM, with the majority of individuals $(72 / 111,64.86 \%)$ revealing association of only two types of the evaluated lesions affecting the inner surface of the skull.

In the 265 out of the 427 selected skeletons with sectioned skulls from the Terry Collection that exhibited at least one type of possible TBM-associated endocranial alterations, PNBFs on the visceral surface of ribs, vertebral hypervascularisation, and vertebral lytic lesions, as well as signs of HPO, extra-spinal TB osteomyelitis and arthritis, and TB cold abscesses, were recorded as non-endocranial bony changes likely related to TB: in $44.15 \%$ (117/265), 43.40\% (115/265), 9.81\% (26/265), 9.43\% (25/265), 6.42\% (17/265), $4.15 \%(11 / 265)$, and $10.57 \%(28 / 265)$ of the aforementioned specimens, respectively. Thus, PNBFs on the visceral surface of ribs and vertebral hypervascularisation affecting the anterior and/or lateral aspects of the bodies represented the most commonly detected types of possible TB-associated non-endocranial bony changes in the 265 selected skeletons with sectioned skulls from the Terry Collection displaying at least one probable TBM-related endocranial alteration type. Moreover, of the 265 skeletons, about one-third (98/265, $36.98 \%$ ) showed no signs of non-endocranial bony changes likely associated with TB; whereas the remaining two-thirds $(167 / 265,63.02 \%)$ of individuals exhibited association of probable TB-related endocranial and non-endocranial bony changes, with the majority $(106 / 167,63.47 \%)$ of skeletons revealing simultaneous occurrence of at least two different types of possible TB-associated non-endocranial bony changes; nevertheless, the number of non-endocranial bony changes probably related to TB and concurrently affecting the abovementioned specimens varied form two to seven.

In summary, at least one type of endocranial alterations likely associated with TBM was registered in about two-thirds $(265 / 427,62.06 \%)$ of the 427 selected skeletons with sectioned skulls from the Terry Collection that were macroscopically evaluated for the presence of the aforementioned lesions affecting the inner surface of the skull, without prior knowledge of the cause of death of individuals. In addition, the co-occurrence of probable TBM-related endocranial alteration types with each other and with possible TB-associated non-endocranial bony changes was detected in about one-fourth $(111 / 427,25.99 \%)$ and more than one-third $(167 / 427,39.11 \%)$ of the surveyed specimens, respectively. 
2) After the detailed macromorphological evaluation of the 427 selected skeletons with sectioned skulls from the Terry Collection, subsequent statistical analysis of data was performed: absolute and percentage frequencies of the four types of endocranial alterations probably related to TBM, as well as of their co-occurrence with each other and with nonendocranial bony changes likely associated with TB, were calculated in both the TB group ( $\Sigma=234)$ and NTB group ( $\Sigma=193)$, considering the sex and age at death of individuals; and $\chi^{2}$ testing of data to determine the significance of differences (if any) in frequencies of the four examined lesion types affecting the inner surface of the skull, as well as of their association with each other and with possible TB-related non-endocranial bony changes between the two groups, was undertaken.

APDIs, PAs, ABVIs, and GIs were registered in both the TB group and NTB group: in $65.81 \%(154 / 234)$ versus $32.12 \%$ (62/193), $20.09 \%$ (47/234) versus $10.36 \%(20 / 193)$, $21.37 \%(50 / 234)$ versus $6.22 \%(12 / 193)$, and $29.06 \%(68 / 234)$ versus $3.11 \%(6 / 193)$ of specimens surveyed, respectively. Therefore, every evaluated endocranial alteration type likely associated with TBM occurred more frequently in the TB group than in the NTB group. Whereas APDIs and PAs affected individuals with TB as the cause of death about twice as often as specimens with NTB causes of death, ABVIs and GIs were approximately three and a half times and ten times more common in individuals recorded to have died of TB than in specimens identified to have died of causes other than TB, respectively. The cooccurrence of the four examined lesion types affecting the inner surface of the skull with each other and with non-endocranial bony changes possibly related to TB were detected in both the TB group and NTB group: in $41.03 \%$ (96/234) versus $7.77 \%(15 / 193)$ and $80.11 \%$ (145/181) versus $26.19 \%$ (22/84) of individuals surveyed, respectively. Thus, the cooccurrence of at least two types of probable TBM-associated endocranial alterations was about five times more frequent in the TB group than in the NTB group; whereas the association of likely TB-related endocranial and non-endocranial bony changes occurred approximately three times more commonly in specimens with TB as the cause of death than in individuals with NTB causes of death.

Furthermore, the $\chi^{2}$ comparison of the frequencies of APDIs, PAs, ABVIs, and GIs, as well as of their co-occurrence with each other and with probable TB-associated nonendocranial bony changes, revealed a statistically significant difference between the TB group and NTB group $\left(\chi^{2}=46.680, \mathrm{df}=1, \mathrm{P}<0.0001 ; \chi^{2}=6.841, \mathrm{df}=1, \mathrm{P}=0.0089 ; \chi^{2}=18.357\right.$, $\mathrm{df}=1, \mathrm{P}<0.0001 ; \chi^{2}=47.922, \mathrm{df}=1, \mathrm{P}<0.0001 ; \chi^{2}=59.079, \mathrm{df}=1, \mathrm{P}<0.0001 ;$ and $\chi^{2}=69.280$, $\mathrm{df}=1, \quad \mathrm{P}<0.0001$, respectively); therefore - similar to the results of previous 
palaeopathological and palaeomicrobiological studies performed on osteoarchaeological series and documented skeletal collections (e.g., Schultz, 1993, 1999, 2001, 2003; Templin \& Schultz, 1994; Teschler-Nicola et al., 1994, 2015; Jankauskas \& Schultz, 1995; Jankauskas, 1999; Hershkovitz et al., 2002; Maczel, 2003; Pálfi et al., 2012; Masson et al., 2015; Molnár et al., 2015; Schultz \& Schmidt-Schultz, 2015) -, constituting evidence that there may be a positive correlation between the above-mentioned lesions and tuberculosis. Thus, APDIs, PAs, ABVIs, and GIs can be used as diagnostic criteria for TBM in the palaeopathological practice. Although not all of them may be considered as specific vestiges of the disease - since pathological conditions other than tuberculosis (e.g., bacterial meningitis, trauma, scurvy, and epidural haematoma) may also result in the development of similar or even the same morphological features -, palaeopathologists could still use them to identify TB in osteoarchaeological material from the pre-antibiotic era, especially when they simultaneously occur with each other and/or with non-endocranial bony changes possibly related to TB.

Considering the sex of the 427 selected skeletons with sectioned skulls from the Terry Collection, APDIs, PAs, ABVIs, and GIs were registered among both females $(\Sigma=65)$ and males $(\Sigma=169)$ in the TB group: in $52.31 \%$ (34/65) versus $71.01 \%(120 / 169), 23.08 \%$ (15/65) versus $18.93 \%$ (32/169), 30.77\% (20/65) versus $17.75 \%(30 / 169)$, and $30.77 \%$ (20/65) versus $28.40 \%$ (48/169) of specimens surveyed, respectively. Similar to the TB group, APDIs, PAs, ABVIs, and GIs were recorded among both females $(\Sigma=87)$ and males $(\Sigma=106)$ in the NTB group: in $21.84 \%$ (19/87) versus $40.57 \%$ (43/106), $10.34 \%(9 / 87)$ versus $10.38 \%(11 / 106), 6.90 \%(6 / 87)$ versus $5.66 \%(6 / 106)$, and $2.30 \%(2 / 87)$ versus $3.77 \%$ (4/106) of individuals examined, respectively. Therefore, every evaluated endocranial alteration type likely associated with TBM occurred more frequently in the TB group than in the NTB group among both females and males. The co-occurrence of the four examined lesion types affecting the inner surface of the skull with each other and with non-endocranial bony changes probably related to TB were detected among both females and males in the TB group: in $43.08 \%$ (28/65) versus $40.24 \%$ (68/169) and $71.11 \%(32 / 45)$ versus $83.09 \%$ (113/136) of specimens surveyed, respectively. Similar to the TB group, the co-occurrence of the four examined lesion types affecting the inner surface of the skull with each other and with non-endocranial bony changes possibly associated with TB were observed among both females and males in the NTB group: in $8.05 \%$ (7/87) versus $7.55 \%(8 / 106)$ and $28.57 \%$ $(8 / 28)$ versus $25.00 \%$ (14/56) of individuals examined, respectively. Thus, the co-occurrence of at least two types of probable TBM-related endocranial alterations, as well as the 
association of endocranial and non-endocranial bony changes likely related to TB, were more commonly registered in specimens with TB as the cause of death than in individuals with NTB causes of death among both females and males.

Moreover, when the two groups were compared considering the sex of the surveyed specimens, the difference in the frequencies of APDIs, ABVIs, and GIs, as well as of the cooccurrence of the four evaluated lesion types affecting the inner surface of the skull with each other and with probable TB-associated non-endocranial bony changes remained significant for both females $\left(\chi^{2}=13.896, \mathrm{df}=1, \mathrm{P}=0.0002 ; \chi^{2}=13.317, \mathrm{df}=1, \mathrm{P}=0.0003\right.$; $\chi^{2}=22.115, \mathrm{df}=1, \mathrm{P}<0.0001 ; \chi^{2}=23.820, \mathrm{df}=1, \mathrm{P}<0.0001 ;$ and $\chi^{2}=10.951, \mathrm{df}=1, \mathrm{P}=0.0009$, respectively) and males $\left(\chi^{2}=23.759, \mathrm{df}=1, \mathrm{P}<0.0001 ; \chi^{2}=7.342, \mathrm{df}=1, \mathrm{P}=0.0067 ; \chi^{2}=24.188\right.$, $\mathrm{df}=1, \mathrm{P}<0.0001 ; \chi^{2}=33.192, \mathrm{df}=1, \mathrm{P}<0.0001 ;$ and $\chi^{2}=57.205, \mathrm{df}=1, \mathrm{P}<0.0001$, respectively); whereas the $\chi^{2}$ comparison of the frequencies of PAs between individuals with TB as the cause of death and specimens with NTB causes of death revealed no statistically significant difference among both females $\left(\chi^{2}=3.629, \mathrm{df}=1, \mathrm{P}=0.0568\right)$ and males $\left(\chi^{2}=2.997, \mathrm{df}=1\right.$, $\mathrm{P}=0.0834$ ). The frequencies of PAs and GIs, as well as of the co-occurrence of the four evaluated lesion types affecting the inner surface of the skull with each other and with likely TB-related non-endocranial bony changes, were very similar among females and males in both the TB group and NTB group; thus, there was no statistically significant difference between the two sexes (TB group: $\chi^{2}=0.277, \mathrm{df}=1, \mathrm{P}=0.5987$ and NTB group: $\chi^{2}=0.0529$, $\mathrm{df}=1, \mathrm{P}=0.8181$; TB group: $\chi^{2}=0.0386, \mathrm{df}=1, \mathrm{P}=0.8443$ and NTB group: $\chi^{2}=0.0291, \mathrm{df}=1$, $\mathrm{P}=0.8645$; TB group: $\chi^{2}=0.0611, \mathrm{df}=1, \mathrm{P}=0.8047$ and NTB group: $\chi^{2}=0.0200, \mathrm{df}=1$, $\mathrm{P}=0.8876$; and TB group: $\chi^{2}=2.339, \mathrm{df}=1, \mathrm{P}=0.1262$ and NTB group: $\chi^{2}=0.00296, \mathrm{df}=1$, $\mathrm{P}=0.9566$, respectively). Nevertheless, in both the TB group and NTB group, an approximately 20 percentage point difference (male predominance: $52.31 \%$ versus $71.01 \%$ and $21.84 \%$ versus $40.57 \%$, respectively) in the frequencies of APDIs was found between females and males; and the $\chi^{2}$ comparison of the frequencies of APDIs between the two sexes revealed a statistically significant difference in both specimens identified to have died of $\mathrm{TB}\left(\chi^{2}=6.487, \mathrm{df}=1, \mathrm{P}=0.0109\right)$ and individuals recorded to have died of causes other than TB $\left(\chi^{2}=6.850, \mathrm{df}=1, \mathrm{P}=0.0089\right)$. Unfortunately, there may be no appropriate scientific explanation for these findings in the two groups, as according to the results of recent medical studies (e.g., Brodsky, 2010; Dendane et al., 2013; Salekeen et al., 2013; Smith et al., 2013; Bir et al., 2016; Tyagi et al., 2016), there may be no statistically significant difference between the two sexes or there may be a slight female or male predominance in adult patients with TBM or with other underlying medical conditions that may result in the development 
of eICP with consequent formation of APDIs on the inner surface of the skull. Furthermore, an about 13 percentage point difference (female predominance: $30.77 \%$ versus $17.75 \%$ ) in the frequencies of ABVIs between females and males was found in the TB group; and the $\chi^{2}$ comparison of the frequencies of ABVIs between the two sexes in the TB group revealed a statistically significant difference $\left(\chi^{2}=3.992, \mathrm{df}=1, \mathrm{P}=0.0457\right)$. Unfortunately, no data were found in the modern medical literature that would appropriately explain the abovementioned findings. Nonetheless, the frequencies of ABVIs among females and males were very similar in the NTB group; therefore, there was no statistically significant difference between the two sexes $\left(\chi^{2}=0.00295, \mathrm{df}=1, \mathrm{P}=0.9567\right)$.

Regarding the distribution of affected specimens by age at death, APDIs occurred with the highest frequency among individuals under the age of 30 years $(61 / 70,87.14 \%)$ : with $88.89 \%$ (56/63) in the TB group and with $71.43 \%$ (5/7) in the NTB group. Similar to the APDIs, PAs, and ABVIs affected most commonly specimens under the age of 30 years in the TB group: more than two-fifths of the aforementioned individuals revealed PAs (26/63, $41.27 \%$ ) or ABVIs $(28 / 63,44.44 \%)$ on the inner surface of the skull. Nevertheless, in the NTB group, the majority of specimens exhibiting PAs $(18 / 20,90.00 \%)$ or ABVIs (11/12, $91.67 \%$ ) were above the age of 40 years. GIs occurred with the highest frequency among individuals under the age of 20 years $(4 / 7,57.14 \%)$ in the TB group; nonetheless, more than one-third $(55 / 158,34.81 \%)$ of specimens between 20 and 49 years of age and with TB as the cause of death also showed GIs on the inner surface of the skull. Similar to the PAs and ABVIs, GIs affected almost exclusively individuals above the age of 40 years $(5 / 6,83.33 \%)$ in the NTB group. As for the co-occurrence of at least two different types of endocranial alterations probably related to TBM, specimens under the age of 30 years $(45 / 63,71.43 \%)$ were most frequently affected by it in the TB group; nevertheless, more than one-third (40/102, 39.22\%) of individuals between 30 and 49 years of age and recorded to have died of TB were also concurrently affected by at least two different types of endocranial alterations possibly associated with TBM. In the NTB group, the vast majority of specimens $(5 / 6,83.33 \%)$ exhibiting co-occurrence of at least two different types of endocranial alterations likely related to TBM were above the age of 40 years. The simultaneous occurrence of probable TB-associated endocranial and non-endocranial bony changes was recorded with the highest frequency among individuals above the age of 40 years (104/119, $87.39 \%)$ : with $89.62 \%(95 / 106)$ and $69.23 \%$ (9/13) in the TB group and NTB group, respectively. The $\chi^{2}$ testing of the frequencies of APDIs, PAs, ABVIs, and GIs, as well as of the co-occurrence of the four evaluated lesion types affecting the inner surface of the skull 
with each other and with likely TB-related non-endocranial bony changes, between the various age groups was not assessed because of the low number of specimens in certain age groups.

3) During the macromorphological evaluation of the 427 selected skeletons with sectioned skulls from the Terry Collection, concerning the observed APDIs, their prominence was registered according to a 3-level scale (very slight: shallow DIs $(<2 \mathrm{~mm}$ in depth) predominantly over the anterior portion of the endocranial surface; slight: deeper DIs (2-4 $\mathrm{mm}$ in depth) particularly over the anterior and middle portions of the endocranial surface; and pronounced: deep DIs (4 $\mathrm{mm}<$ in depth) all over the endocranial surface); whereas regarding the detected PAs, ABVIs, and GIs, the affected cranial bone(s); the number of recorded lesions in the affected cranial bone(s) (unifocal or multifocal); and the extent of the endocranial surface area the observed lesion(s) covered (x) in the affected cranial bone(s) (4-level scale: 1) $\mathrm{x}<25 \%$, 2) $25 \% \leq \mathrm{x}<50 \%$, 3) $50 \% \leq \mathrm{x}<75 \%$, and 4) $75 \% \leq \mathrm{x})$ were noted.

As for the prominence of APDIs detected in 216 out of the 427 selected skeletons with sectioned skulls from the Terry Collection, very slight APDIs occurred in more than twothirds $(148 / 216,68.52 \%)$ of the above-mentioned individuals. Whereas very slight APDIs were more commonly registered in specimens with NTB causes of death, slight and pronounced APDIs were more frequently recorded in individuals with TB as the cause of death: in $62.34 \%$ (96/154) versus $83.87 \%$ (52/62), $27.27 \%$ (42/154) versus $14.52 \%$ (9/62), and $10.39 \%(16 / 154)$ versus $1.61 \%(1 / 62)$, respectively.

Regarding the localisation of PAs and ABVIs on the inner surface of the skull, the frontal bone (predominantly its most protruding portions and/or orbital part) and the parietal bones (particularly their most protruding portion and/or their part along the superior sagittal sinus) represented the most common sites of involvement in both the TB group and NTB group. Occasionally, the occipital bone (generally its squamous part along the superior sagittal and/or transverse sinuses), the temporal bones (mostly their squamous part), and the greater wings of the sphenoid bone were also affected. Considering the left and right greater wings of the sphenoid bone as two separate bones, in more than one-half of specimens with TB as the cause of death and PAs or ABVIs, at least three or four cranial bones were simultaneously affected: in 53.19\% (25/47) and 64.00\% (32/50), respectively; whereas in the majority of individuals with NTB causes of death and PAs or ABVIs, less than three or four cranial bones were concomitantly involved: in 65.00\% (13/20) and 75.00\% (9/12), 
respectively. In contrast to the PAs and ABVIs, GIs occurred most frequently on the squamous part of the occipital bone in both the TB group and NTB group. Moreover, GIs were quite often observed in the orbital part of the frontal bone and in the squamous part of the temporal bones; whereas involvement of the greater wings of the sphenoid bone, as well as of the parietal bones (predominantly along the squamous suture) by GIs, was registered in only the minority of skeletons surveyed. In both the TB group and NTB group, less than four cranial bones (considering the left and right greater wings of the sphenoid bone as two separate bones) were concurrently affected by GIs in approximately two-thirds of specimens exhibiting the aforementioned probable TBM-associated endocranial alteration type: in $72.06 \%(49 / 68)$ and $66.67 \%(4 / 6)$, respectively.

Concerning the extent of the detected lesions, the majority of PAs and ABVIs (observed in 67 and 62 out of the 427 selected skeletons with sectioned skulls from the Terry Collection, respectively) covered less than one-half of the endocranial surfaces in all cranial bones examined. Nonetheless, the extent of PAs and ABVIs recorded in the frontal and parietal bones exceeded one-half of the inner surfaces quite frequently. In contrast to the PAs and ABVIs, the majority of GIs registered in 74 out of the 427 selected skeletons with sectioned skulls from the Terry Collection covered only less than one-fourth of the endocranial surfaces in all cranial bones examined. Nevertheless, the extent of GIs detected in the sphenoid and temporal bones exceeded one-fourth of the inner surfaces quite commonly. With respect to the number of lesions, in the 67 and 62 cases exhibiting PAs and ABVIs, respectively, the above-mentioned endocranial alterations likely related to TBM occurred almost exclusively as multifocal bony changes on the inner surface of the skull in all cranial bones evaluated, and only occasionally were observed as unifocal lesions. Similar to the PAs and ABVIs, in the 74 affected cases, GIs were particularly recorded as multifocal bony changes in the frontal and occipital bones; nonetheless, they were registered as unifocal alterations in the parietal, sphenoid, and temporal bones quite often.

In summary, in more than two-thirds of 216 out of the 427 selected skeletons with sectioned skulls from the Terry Collection that exhibited APDIs, the very slight stage of the aforementioned lesion was detected. As for the localisation of PAs, ABVIs, and GIs, findings of the current $\mathrm{PhD}$ dissertation were similar to those of previous studies (e.g., Mensforth $e t$ al., 1978; Schultz, 1993, 1999; Hershkovitz et al., 2002; Schultz \& Schmidt-Schultz, 2015): PAs and ABVIs were situated particularly on the inner surface of the frontal and parietal bones, with their most protruding portion and/or part along the superior sagittal sinus being the most commonly affected areas; whereas GIs were localised predominantly on the 
endocranial surface of the skull base and lateral skull vault, mostly on the squamous part of the occipital and temporal bones, as well as on the orbital part of the frontal bone, with the pattern and distribution of GIs resembling that of most often observed in the affected meninges during the pathogenesis of TBM (Fig. 76A-F). Considering the left and right greater wings of the sphenoid bone as two separate bones, in more than one-half of individuals with TB as the cause of death and PAs or ABVIs, at least three or four cranial bones were simultaneously affected, respectively; whereas in the majority of specimens with NTB causes of death and PAs or ABVIs, less than three or four cranial bones were concomitantly involved, respectively. In both groups, less than four cranial bones were concurrently affected by GIs in about two-thirds of individuals exhibiting the abovementioned probable TBM-associated endocranial alteration type. Regarding the extent of lesions, whereas the majority of the detected PAs and ABVIs covered less than one-half of the endocranial surfaces in all cranial bones examined, the extent of the endocranial surface area the observed GIs covered in the affected cranial bones only occasionally exceeded onefourth of the inner surfaces. Concerning the number of lesions, PAs, ABVIs, and GIs were registered most frequently as multifocal alterations in all cranial bones evaluated.

4) In the present $\mathrm{PhD}$ dissertation (at the end of each section of the RESULTS \& CASE STUDIES chapter), example cases were provided that showed the most important macromorphological characteristics of the four evaluated endocranial alteration types probably related to TBM, as well as of their co-occurrence with each other and/or with nonendocranial bony changes likely associated with TB. Terry No. 30R, 382R, and 1033 were used to represent the very slight, slight, and pronounced stages of the prominence of APDIs, respectively. The most characteristic localisations of PAs, ABVIs, and GIs on the inner surface of the cranial bones were illustrated by Terry No. 987, 1027, and 1300; Terry No. 254, 1030, and 1555; and Terry No. 522, 562, 566, and 933R, respectively. Six further example cases were used to demonstrate the co-occurrence of the four possible TBM-related endocranial alteration types with each other (Terry no. 304, 1159, 1222, and 1322) and with non-endocranial bony changes probably associated with TB (Terry No. 468 and 902). The detailed case studies presented in the current $\mathrm{PhD}$ dissertation may give a better insight into the macromorphological characteristics of the examined lesions affecting the inner surface of the skull, and may provide palaeopathologists with a stronger basis for establishing a more reliable and accurate diagnosis of TBM in ancient human bone remains that exhibit bony changes resembling those of the example cases presented here. 
Figure 76: Typical localisations of PAs, ABVIs, and GIs on the inner surface of the skullcap: A), C), and E), respectively; and of the skull base: B), D), and F), respectively (blue: most commonly affected areas, orange: commonly affected areas, and yellow: less commonly affected areas) (drawings by Luca Kis).

A)



C)



E)

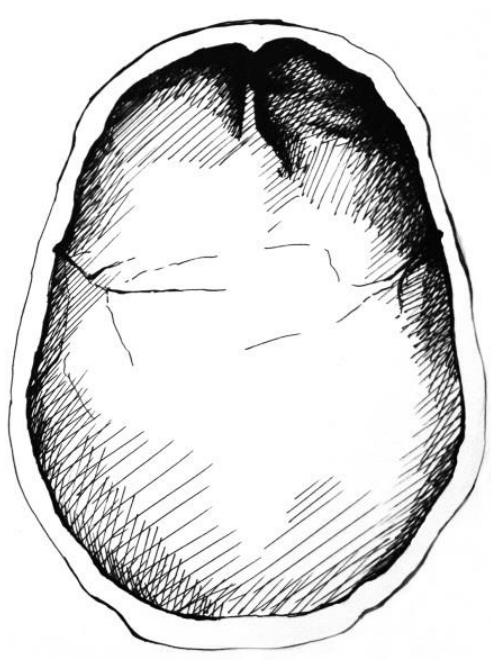

B)

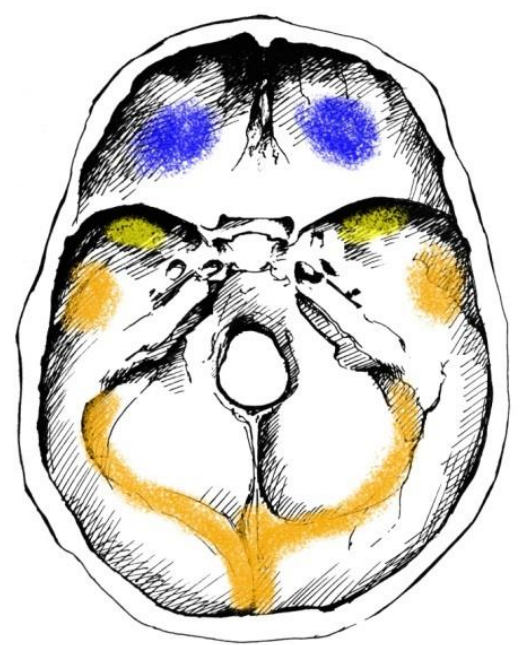

D)



F)




5) On the basis of the findings obtained from the research project performed on the 427 selected skeletons with sectioned skulls from the Terry Collection, the four evaluated endocranial alteration types (i.e., APDIs, PAs, ABVIs, and GIs) can be used as diagnostic criteria for TBM in the palaeopathological practice, as all of them occurred more frequently in the TB group than in the NTB group; and the $\chi^{2}$ comparison of the frequencies of APDIs, PAs, ABVIs, and GIs, as well as of their co-occurrence with each other and with likely TBrelated non-endocranial bony changes, revealed a statistically significant difference between the two groups; thus, indicating that there may be a positive correlation between the examined lesions and TBM. However, the diagnostic value of the aforementioned endocranial alteration types is not equal.

In comparison with the three other endocranial alteration types possibly associated with TBM (i.e., APDIs, ABVIs, and GIs), PAs have the weakest diagnostic value in the palaeopathological identification of TBM in ancient human bone remains based on the findings of the present $\mathrm{PhD}$ dissertation, since even if they were detected in specimens with TB as the cause of death about twice as often as in individuals with NTB causes of death, the $\chi^{2}$ comparison of the frequencies of PAs revealed a statistically only significant difference between the TB group and NTB group $\left(\chi^{2}=6.841, \mathrm{df}=1, \mathrm{P}=0.0089\right)$, suggesting a weaker correlation between PAs and TBM. Furthermore, the results obtained from the research project performed on the 427 selected skeletons with sectioned skulls from the Terry Collection may support those of previous studies (e.g., Mensforth et al., 1978; Schultz, 1993, 1999, 2001, 2003) regarding the specificity of the above-mentioned endocranial alteration type for TBM, as PAs were observed in more than $10 \%(20 / 193)$ of the 193 skeletons composing the NTB group; and thus, indicating that they cannot be considered as specific vestiges of TBM. In one-half $(10 / 20,50.00 \%)$ of the specimens with PAs in the NTB group, probable TBM-related endocranial alterations other than PAs (APDIs: eight cases, ABVIs: one case, and GIs: one case) and/or likely TB-associated non-endocranial bony changes (PNBFs: one case and vertebral hypervascularisation: one case) were also noted. Since the disease registered as the cause of death on the morgue record and/or death certificate may not have been the only medical condition present in the individuals surveyed in the Terry Collection, specimens identified to have died of causes other than TB could still have suffered from tuberculosis at death (Roberts et al., 1994; Santos \& Roberts, 2001). Thus, in the aforementioned ten individuals with PAs in the NTB group, the tuberculous origin of the recorded endocranial and non-endocranial lesions cannot be excluded. However, it is also possible that in these ten cases - similar to the other one-half (10/20, 
$50.00 \%$ ) of specimens with PAs in the NTB group, where no signs of probable TBM-related endocranial alterations other than PAs or of likely TB-associated non-endocranial bony changes were observed -, medical conditions other than TB (e.g., bacterial meningitis, trauma, and scurvy) may result in the development of the detected lesions.

On the basis of the findings obtained from the research project performed on the 427 selected skeletons with sectioned skulls from the Terry Collection, APDIs have a stronger diagnostic value in the palaeopathological identification of TBM in past human populations than PAs, since - similar to the PAs - APDIs were registered in individuals recorded to have died of TB approximately twice as frequently as in specimens identified to have died of causes other than TB; but - in contrast to the PAs - the $\chi^{2}$ comparison of the frequencies of APDIs revealed a statistically not only significant but extremely significant difference between the TB group and NTB group $\left(\chi^{2}=46.680, \mathrm{df}=1, \mathrm{P}<0.0001\right)$; therefore, indicating a strong correlation between APDIs and TBM. Nevertheless, the results of the present PhD dissertation fit in with those of previous studies (e.g., Schultz, 1993, 1999, 2001, 2003) concerning the specificity of the above-mentioned endocranial alteration type for TBM, as about one-third $(62 / 193,32.12 \%)$ of the 193 skeletons constituting the NTB group revealed APDIs on the inner surface of the skull; thus, suggesting that APDIs cannot be considered as pathognomonic features of TBM. In less than one-half $(28 / 62,45.16 \%)$ of the individuals with APDIs in the NTB group, probable TBM-related endocranial alterations other than APDIs (PAs: seven cases, ABVIs: three cases, and GIs: four cases) and/or likely TBassociated non-endocranial bony changes (PNBFs: four cases, vertebral hypervascularisation: 12 cases, vertebral lytic lesions: three cases, signs of extra-spinal osteomyelitis: three cases, signs of HPO: four cases, and signs of cold abscesses: two cases) were also registered. It must be noted that even if the recorded cause of death of specimens surveyed in the Terry Collection may not have been tuberculosis, individuals could still have suffered from the disease but their death was attributed to another medical condition (Roberts et al., 1994; Santos \& Roberts, 2001); therefore, it cannot be excluded that in the aforementioned 28 specimens with APDIs in the NTB group, the observed endocranial and non-endocranial bony changes might result from TB. However, in the other one-half (34/62, $54.84 \%$ ) of individuals with APDIs in the NTB group, where no signs of probable TBMrelated endocranial alterations other than APDIs or of possible TB-associated nonendocranial bony changes were detected, the NTB origin (e.g., other CNS infections, brain tumours, and haemorrhages) of the noted lesions is much more likely. 
In comparison with the APDIs and PAs, ABVIs have a stronger diagnostic value in the palaepathological identification of TBM in osteoarchaeological material from the preantibiotic era based on the findings of the current $\mathrm{PhD}$ dissertation, since they were about three and a half times more common in specimens with TB as the cause of death than in individuals with NTB causes of death; and the $\chi^{2}$ comparison of the frequencies of ABVIs revealed a statistically extremely significant difference between the TB group and NTB group $\left(\chi^{2}=18.357, \mathrm{df}=1, \mathrm{P}<0.0001\right)$; therefore, suggesting a strong correlation between ABVIs and TBM. Nonetheless, the results of the research project conducted on the 427 selected skeletons with sectioned skulls from the Terry Collection may support those of previous studies (e.g., Schultz, 1993, 1999, 2001, 2003) regarding the specificity of the above-mentioned endocranial alteration type for TBM, as ABVIs were observed in more than $6 \%$ (12/193) of the 193 skeletons composing the NTB group; thus, indicating that they cannot be considered as specific vestiges of TBM. In more than one-half $(7 / 12,58.33 \%)$ of the individuals with ABVIs in the NTB group, probable TBM-related endocranial alterations other than ABVIs (APDIs: three cases and PAs: two cases) and/or likely TB-associated nonendocranial bony changes (PNBFs: one case, signs of extra-spinal osteomyelitis: one case, signs of HPO: one case, and signs of cold abscesses: one case) were also noted. Since the disease registered as the cause of death on the morgue record and/or death certificate may not have been the only medical condition present in the specimens surveyed in the Terry Collection, individuals recorded to have died of causes other than TB could still have suffered from tuberculosis at death (Roberts et al., 1994; Santos \& Roberts, 2001). Therefore, in the aforementioned seven specimens with ABVIs in the NTB group, the tuberculous origin of the detected endocranial and non-endocranial lesions cannot be excluded. However, it is also possible that in these seven cases - similar to the other onehalf $(5 / 12,41.67 \%)$ of individuals with ABVIs in the NTB group, where no signs of probable TBM-related endocranial alterations other than ABVIs or of likely TB-associated nonendocranial bony changes were observed -, medical conditions other than TB (e.g., bacterial meningitis, trauma, and scurvy) may result in the development of the observed lesions, since most of them cannot be considered as pathognomonic features of TB.

On the basis of the results obtained from the research project performed on the 427 selected skeletons with sectioned skulls from the Terry Collection, among the four possible TBM-related endocranial lesion types, GIs have the strongest diagnostic value in the palaeopathological identification of TBM in past human populations, since they occurred approximately ten times more often in specimens with TB as the cause of death than in 
individuals with NTB causes of death; and the $\chi^{2}$ comparison of the frequencies of GIs revealed a statistically extremely significant difference between the TB group and NTB group $\left(\chi^{2}=47.922, \mathrm{df}=1, \mathrm{P}<0.0001\right)$. Moreover, the findings of the present $\mathrm{PhD}$ dissertation fit in with those of previous studies (e.g., Schultz, 1999, 2001, 2003; Schultz \& SchmidtSchultz, 2015) concerning the specificity of the above-mentioned endocranial alteration type for TBM, as - in contrast to the previously discussed lesions affecting the inner surface of the skull (i.e., APDIs, PAs, and ABVIs) - GIs affected only the minority (6/193, 3.11\%) of the 193 skeletons constituting the NTB group, and in the vast majority of the abovementioned six specimens $(5 / 6,83.33 \%)$, probable TBM-associated endocranial alterations other than GIs (APDIs: four cases and PAs: one case) and/or likely TB-related nonendocranial bony changes (PNBFs: two cases, vertebral hypervascularisation: two cases, signs of extra-spinal osteomyelitis: two cases, and signs of HPO: one case) were also recorded. It must be noted that even if the registered cause of death of individuals surveyed in the Terry Collection may not have been tuberculosis, specimens could still have suffered from the disease but their death was attributed to another medical condition (Roberts et al., 1994; Santos \& Roberts, 2001); thus, it is very likely that in the aforementioned six cases, the observed endocranial and non-endocranial bony changes might result from TB.

In summary, even if PAs, APDIs, and ABVIs cannot be considered as pathognomonic features of TBM, palaeopathologists could still use them - with necessary circumspection to identify the disease in human osteoarchaeological material from the pre-antibiotic era, especially when they simultaneously occur with each other and/or with possible TB-related non-endocranial bony changes. Furthermore, the results of the current $\mathrm{PhD}$ dissertation may confirm those of Schultz (e.g., 1999, 2001, 2003) and Schmidt-Schultz (2015) that GIs can be considered as pathognomonic features of TBM; and therefore, the palaeopathological diagnosis of TBM can be established with a high certainty when GIs are present in ancient human bone remains. 
6 RESEARCH SIGNIFICANCE \& PERSPECTIVES 
Despite significant advances in the global fight against tuberculosis, it still presents a health emergency; therefore, a renewed interest and funding to the research of the disease and of its aetiological agents has sparked during the last few decades in order to eliminate or at least control TB in the future. Since the beginning of the $21^{\text {st }}$ century, a number of molecular evolutionary studies (e.g., Brosch et al., 2002; Mostowy et al., 2002; Gutierrez et al., 2005; Brisse et al., 2006; Huard et al., 2006; Smith, 2006; Hershberg et al., 2008; Wirth et al., 2008; Comas et al., 2013; Bos et al., 2014) have improved our knowledge on the origin and evolutionary history of the MTBC, as well as on the co-evolution of its members with the human and various wild and domesticated animal hosts; however, the results of the aforementioned research projects are unfortunately insufficient and controversial for the time being (Stone et al., 2009; Gagneux, 2012; Coscolla \& Gagneux, 2014; Galagan, 2014; Bañuls et al., 2015; Brites \& Gagneux, 2015; Pálfi et al., 2015; Pai et al., 2016; WHO, 2017).

In addition, palaeomicrobiological analyses of biological remains of TB bacteria extracted from skeletons and mummies of people lived in the past (e.g., DNA, proteins, and lipid biomarkers) (e.g., Spigelman \& Lemma, 1993; Salo et al., 1994; Arriaza et al., 1995; Haas et al., 2000; Donoghue et al., 2005; Zink et al., 2007; Chan et al., 2013; Hershkovitz et al., 2015; Schmidt-Schultz \& Schultz, 2015) have provided invaluable novel data not only on the evolution of tuberculosis but also on its palaeoepidemiology throughout prehistoric and historic times. Nevertheless, findings of recent palaeoepidemiological studies on human osteoarchaeological series from the pre-antibiotic era (e.g., Masson et al., 2015; Molnár et al., 2015; Pósa et al., 2015) have confirmed the complementarity of palaeomicrobiological and traditional, macromorphology-based palaeopathological analyses, as their combined application may contribute to facilitating the establishment of a more reliable and accurate palaeopathological diagnosis of TB in ancient human bone remains and the assessment of a more relevant prevalence of the disease in past human populations (Stone et al., 2009; Donoghue et al., 2015; Minnikin et al., 2015; Pálfi et al., 2015).

The above-mentioned examinations require excessive scientific knowledge on the macromorphological diagnostics of tuberculosis that underlines the importance of the present research project conducted on skeletons of known cause of death from the Terry Collection, since its results may provide palaeopathologists with a stronger basis for identifying TB and with a more sensitive means of assessing the prevalence of the disease in human osteoarchaeological material. Nonetheless, further investigations on human skeletons of known cause of death from documented collections other than the Terry Collection are necessary to confirm the trends noted in the current $\mathrm{PhD}$ dissertation. 
Refinement of macromorphological diagnostic criteria and their application in the palaeopathological practice may open new perspectives in the palaeoepidemiological and evolutionary research of tuberculosis.

Finally, findings of the present $\mathrm{PhD}$ dissertation may draw physicians' attention to the rather high prevalence of meningeal involvement in TB patients; and thus, may contribute to further improving the modern medical practice regarding the identification of TBM. According to the modern medical literature (Garg, 1999; Bernaerts et al., 2003; Katti, 2004; Gauba \& Varma, 2005; Bill, 2006; Myers, 2007; Rock et al., 2008; Thwaites et al., 2009; Cherian \& Thomas, 2011; Christensen et al., 2011; Bano et al., 2012; Thwaites, 2013; Bini \& Hernández Pando, 2014; Daniele, 2014; Taheri et al., 2015; Tyagi et al., 2016; Chaudhary et al., 2017; Vita et al., 2017), TBM - the most common form of CNS TB ( 70-80\%) occurs in less than $1 \%$ of all active TB cases, with children under the age of 5 years representing the most vulnerable group affected by the disease. Although - because of the particular composition of the Terry Collection - there were no children among the evaluated skeletons with sectioned skulls (Suppl. table 1), probable TBM-related endocranial alterations and their co-occurrence with each other and/or with likely TB-associated nonendocranial bony changes were observed in a number of adolescent and adult individuals with TB as the cause of death from the Terry Collection. It must be noted that the vast majority of the aforementioned specimens were identified to have died of pulmonary TB and only two of them were recorded to have died of TBM (Suppl. table 1).

Nevertheless, results of the current research project fit in with those of recent studies revealing that at autopsy, a large number of individuals died of pulmonary TB without developing neurological signs and symptoms exhibited tubercles in the CNS; and therefore, indicating that involvement of the central nervous system in pulmonary TB is quite common. The above-mentioned findings may incite physicians to check pulmonary TB patients for involvement of the CNS even if they do not display neurological signs and symptoms suggestive of the disease, since early, accurate diagnosis and prompt, adequate treatment are crucial in determining the clinical outcome of TBM that carries a high short-term mortality and a substantial excess morbidity among survivors: approximately one-third of the affected patients die of TBM and up to one-half of the survivors remain with serious neurological sequels, despite the initiation of anti-tuberculosis therapy (Thwaites et al., 2002; Gauba \& Varma, 2005; Garg, 2010; Christensen et al., 2011; Marx \& Chan, 2011; Brancusi et al., 2012; Bini \& Hernández Pando, 2014; Daniele, 2014; Rajashekar et al., 2014; RamirezLapausa et al., 2015; Vita et al., 2017). 
7 ACKNOWLEDGEMENTS 
Although the current $\mathrm{PhD}$ dissertation is an individual work, it would not have come to a successful completion without the guidance, encouragement, support, help, and efforts of a lot of people. It is my genuine pleasure to acknowledge all of them.

First and foremost, I would like to express my sincere gratitude to my $\mathrm{PhD}$ supervisors, Erika Molnár, PhD, assistant professor (Department of Biological Anthropology, University of Szeged, Szeged, Hungary) and György Pálfi, PhD, associate professor and head of department (Department of Biological Anthropology, University of Szeged, Szeged, Hungary), whose valuable guidance, scholarly inputs, consistent encouragement, and continuous support I received throughout my $\mathrm{PhD}$ studies and related research made it possible for me to work on a topic that was of a great interest to me.

Besides my supervisors, I wish to extend my deepest sense of gratitude and indebtedness to David R. Hunt, PhD, collection manager and head of department (Department of Anthropology, National Museum of Natural History, Smithsonian Institution, Washington, DC, USA), who gave access to the laboratory and research facilities, and who in spite of being extraordinary busy with his duties, took time out to hear, guide, and help me during my four-month-long stay in Washington, DC, as well as since then. I could not have imagined having a better external advisor, and without his precious support, it would not have been possible to carry out my $\mathrm{PhD}$ research project in the Terry Collection.

My sincere thanks also go to Prof. Dr. Michael Schultz, MD, PhD, who provided me with an opportunity to visit his laboratory at the Institut für Anatomie und Embryologie (Universitätsmedizin Göttingen, Göttingen, Germany) in August 2017, and who introduced me to palaeohistopathology of bones. It has been a great honour and privilege for me to learn from his research expertise.

In regards to the statistical analysis of data, I am deeply indebted and grateful to László Paja, PhD and Zoltán Pintér, PhD for their contributions, efforts, and help.

These acknowledgements would not be complete without mentioning my friends and research laboratory colleagues, namely Zsolt Bereczki, PhD, Orsolya Anna Váradi, Kitty Király, Luca Kis, William Berthon, Balázs Tihanyi, and János Rovó, who have contributed immensely to my personal and professional time at the Department of Biological Anthropology (University of Szeged, Szeged, Hungary), and who incented and aided me to strive towards my goals. Thank you for the stimulating discussions, for the sleepless nights we were working together before deadlines, and for all the great times that we have shared in the last few years. 
I also owe a lot to Dávid Rakk, an out-of-field colleague from the Department of Microbiology (University of Szeged, Szeged, Hungary), who read my dissertation and provided insightful and constructive comments on it.

I gratefully acknowledge the financial support provided by the Tempus Public Foundation, Hungarian State Eötvös Fellowship, for funding of the $\mathrm{PhD}$ research project I completed in the Terry Collection (National Museum of Natural History, Smithsonian Institution, Washington, DC, USA) between September and December 2016. My PhD work was also supported by the Hungarian Ministry of Human Capacities, Human Capacities Grant Management Office, NTP-NFTÖ-16 from January to June 2017.

Finally, but by no means least, I would like to thank my parents and sister, who encouraged and helped me at every stage of my personal and academic life. Words cannot express how grateful I am to you for all the sacrifices that you have made on my behalf. I dedicate this $\mathrm{PhD}$ dissertation to the memory of my beloved grandfather, István Vagdalt, whose role in my life was, and remains, immense. Thank you.

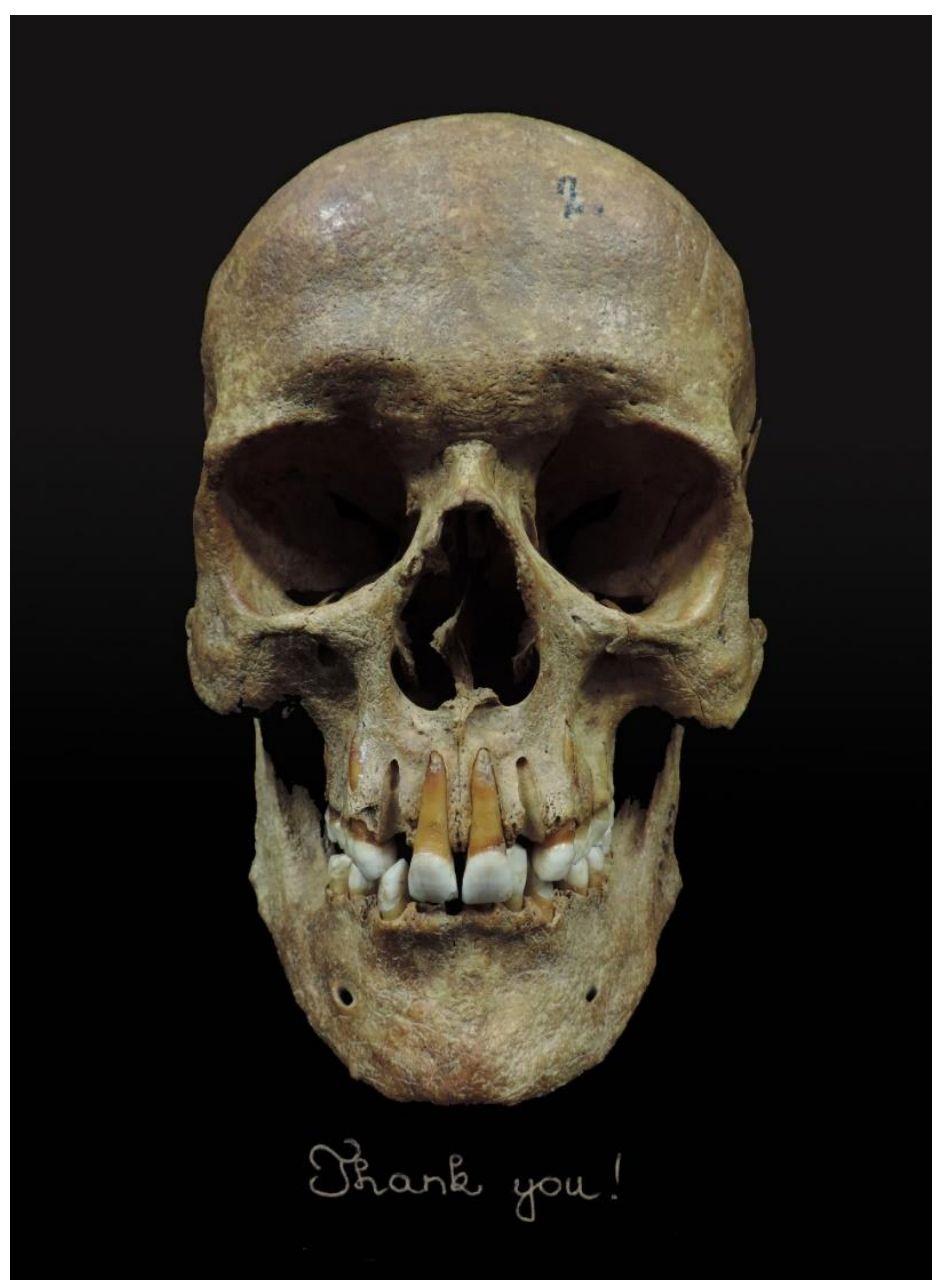

Bácsalmás - Homokbánya, Hungary, $16^{\text {th }}-17^{\text {th }}$ century AD, Grave No. 2, Adultus, male (photo by László Paja). 
8 SUMMARY 
Tuberculosis (TB) is one of the oldest known infectious diseases that has been plaguing mankind for thousands of years. Using modern medical knowledge, palaeopathologists attempt to establish a retrospective diagnosis of prehistoric and historic TB cases by identifying pathological conditions in bone remains of people lived in the past that may be related to the disease. Nevertheless, on the one hand, probable TB-associated bony changes observed in recent cases may differ from those of detectable in skeletons from archaeological sites, due in part to the introduction of antibiotics in the treatment of TB. On the other hand, in modern clinical cases, bony lesions cannot be directly surveyed with macromorphological methods but with medical imaging techniques only; nonetheless, subtle bony alterations probably related to TB may be impossible to be visualised by the latter ones. Therefore, these bony changes are not relevant to the diagnosis of TB in recent cases and are not described as diagnostic criteria for the disease by physicians in the modern medical literature, even if they could be potentially important elements of TB identification for palaeopathologists. Thus, utilisation of modern diagnostic criteria for $\mathrm{TB}$ in the palaeopathological practice may not be appropriate.

Nevertheless, detailed analysis of well-documented collections of pre-antibiotic era skeletons of known cause of death can serve as a unique and important basis for the diagnosis of TB in past human populations, since bone remains of specimens identified to have died of TB and not treated with antibiotics may exhibit similar TB-associated bony lesions to those of observable in skeletons from archaeological sites; they can be directly surveyed with macromorphological methods; and even subtle bony alterations can be recognised in them. Therefore, examination of such collections can contribute to determining the appropriate palaeopathological diagnostic criteria for TB. Since the late $20^{\text {th }}$ century, a number of palaeopathological and palaeomicrobiological studies were performed on osteoarchaeological series and documented skeletal collections that have revealed a correlation between tuberculous meningitis (TBM) and different endocranial alteration types, namely abnormally pronounced digital impressions (APDIs), periosteal appositions (PAs), abnormal blood vessel impressions (ABVIs), and granular impressions (GIs). However, the diagnostic value of the aforementioned lesions has more recently been questioned, as their precise aetiology is still a matter of controversy, and additionally, similar or even the same morphological features can be found in non-TB-related cases.

The main aim of the current PhD dissertation was to expand knowledge and understanding about the development of possible TBM-associated endocranial alterations and to improve their palaeopathological interpretation, as well as to contribute to 
strengthening their diagnostic value in the identification of TB in human osteoarchaeological material from the pre-antibiotic era. Thus, review of the modern medical and palaeopathological literature regarding TB was conducted, with special attention to bony changes likely related to the disease. Furthermore, for the first time, a detailed investigation focusing on the macromorphological characteristics and frequencies of the above-mentioned lesions affecting the inner surface of the skull, as well as of their co-occurrence with each other and with non-endocranial bony changes probably associated with $\mathrm{TB}$, was performed on all individuals recorded to have died of different types of TB (TB group) in the Robert $J$. Terry Anatomical Skeletal Collection, and on a control group (non-tuberculous (NTB) group) consisting of randomly selected specimens from the remaining skeletons of the Terry Collection, identified to have died of causes other than TB.

\section{The objectives of the present $\mathrm{PhD}$ dissertation are the following:}

1) To macroscopically evaluate the selected skeletons for the presence of APDIs, PAs, ABVIs, and GIs, as well as for their co-occurrence with each other and with nonendocranial bony changes possibly related to TB;

2) To compare the frequencies of APDIs, PAs, ABVIs, and GIs, as well as of their cooccurrence with each other and with non-endocranial bony changes likely associated with TB, between the TB group and NTB group, considering the sex and age at death of individuals;

3) To macromorphologically characterise APDIs, PAs, ABVIs, and GIs detected in the examined skeletons regarding the prominence (APDIs), as well as the localisation, extent, and number (PAs, ABVIs, and GIs) of lesions in the affected cranial bone(s);

4) To provide example cases showing the most important macromorphological characteristics of APDIs, PAs, ABVIs, and GIs; and

5) To evaluate the diagnostic value of APDIs, PAs, ABVIs, and GIs concerning the palaeopathological identification of TB.

\section{The main results of the current $\mathrm{PhD}$ dissertation are the following:}

1) As for the presence of APDIs, PAs, ABVIs, and GIs, at least one of them was registered in approximately two-thirds of the selected skeletons from the Terry Collection, with APDIs representing the most frequently detected lesion type and with PAs, ABVIs, and GIs occurring in smaller but similar proportions of specimens. In addition, the association of APDIs, PAs, ABVIs, and GIs with each other and/or with probable TB-related nonendocranial bony changes was noted in about one-third of the surveyed individuals. 
2) APDIs, PAs, ABVIs, and GIs were registered in both the TB group and NTB group; nonetheless, all of them were recorded more commonly in specimens with TB as the cause of death than in individuals with NTB causes of death. Whereas APDIs and PAs occurred in the TB group about twice as often as in the NTB group, ABVIs and GIs were approximately three and a half times and ten times more frequent in specimens with TB as the cause of death than in individuals with NTB causes of death, respectively. Moreover, the cooccurrence of APDIs, PAs, ABVIs, and GIs with each other was about five times more common in the TB group than in the NTB group; whereas their association with likely TBrelated non-endocranial bony changes occurred approximately three times more often in specimens with TB as the cause of death than in individuals with NTB causes of death. The $\chi^{2}$ comparison of the frequencies of APDIs, PAs, ABVIs, and GIs, as well as of their cooccurrence with each other and with probable TB-associated non-endocranial bony changes, revealed a statistically significant difference between the TB group and NTB group; therefore, similar to the results of previous studies, constituting evidence that there may be a correlation between APDIs, PAs, ABVIs, and GIs and TBM. Thus, they can be used as diagnostic criteria for TBM in the palaeopathological practice.

3) With respect to the macromorphological characteristics of APDIs, PAs, ABVIs, and GIs, in more than two-thirds of the selected skeletons from the Terry Collection that showed APDIs, the very slight stage of the aforementioned lesion was detected. As for the localisation of PAs, ABVIs, and GIs, findings of the current PhD dissertation were similar to those of previous studies: PAs and ABVIs were situated particularly in the frontal and parietal bones; whereas GIs were localised predominantly in the occipital and temporal bones, with the pattern and distribution of GIs resembling that of most frequently observed in the affected meninges during the pathogenesis of TBM. Regarding the extent of lesions, whereas the majority of the detected PAs and ABVIs covered less than one-half of the inner surfaces in all cranial bones examined, the extent of the endocranial surface area the observed GIs covered in the affected cranial bones only occasionally exceeded one-fourth of the inner surfaces. Concerning the number of lesions, PAs, ABVIs, and GIs were registered most commonly as multifocal alterations in all cranial bones evaluated.

4) In the present $\mathrm{PhD}$ dissertation, a number of example cases were provided that showed the most important macromorphological characteristics of APDIs, PAs, ABVIs, and GIs (i.e., APDIs: prominence; PAs, ABVIs, and GIs: localisation, extent, and number of lesions in the affected cranial bone(s)), as well as of their co-occurrence with each other and/or with likely TB-related non-endocranial bony changes. Therefore, they may give a better insight 
into the macromorphological characteristics of the above-mentioned lesions, and may provide palaeopathologists with a stronger basis for establishing a more reliable and accurate diagnosis of TBM in ancient human bone remains that exhibit bony changes resembling those of the example cases presented in the current $\mathrm{PhD}$ dissertation.

5) On the basis of the results obtained from the research project performed on selected skeletons from the Terry Collection, the diagnostic value of APDIs, PAs, ABVIs, and GIs in the palaeopathological identification of TBM is not equal. PAs may have the weakest diagnostic value. Even if they were detected in the TB group about twice as often as in the NTB group, the $\chi^{2}$ comparison of the frequencies of PAs revealed a statistically only significant difference between the two groups; thus, suggesting a weaker correlation between PAs and TBM in comparison with APDIs, ABVIs or GIs. APDIs may have a stronger diagnostic value than PAs, since - similar to the PAs - they were registered in the TB group approximately twice as frequently as in the NTB group; but - in contrast to the PAs - the $\chi^{2}$ comparison of the frequencies of APDIs revealed a statistically not only significant but extremely significant difference; therefore, indicating a stronger correlation between APDIs and TBM than between PAs and TBM. In comparison with PAs and APDIs, ABVIs may have a stronger diagnostic value, as they were about three and a half times more common in the TB group than in the NTB group; and - similar to the APDIs - the $\chi^{2}$ comparison of the frequencies of ABVIs revealed a statistically extremely significant difference between the two groups; thus, suggesting a stronger correlation between ABVIs and TBM than between PAs or APDIs and TBM. Nevertheless, the findings of the present PhD dissertation fit in with those of previous studies concerning the specificity of PAs, APDIs, and ABVIs for TBM, as more than $10 \%, 30 \%$, and $6 \%$ of the skeletons constituting the NTB group exhibited them on the inner surface of the skull, respectively; therefore, indicating that PAs, APDIs, and ABVIs cannot be considered as specific vestiges of TBM. Nonetheless, in about onehalf of the specimens with PAs, APDIs or ABVIs in the NTB group, other probable TBMrelated endocranial alterations and/or likely TB-associated non-endocranial bony changes were also noted. Since the disease registered as the cause of death on the morgue record and/or death certificate may not have been the only medical condition present in the surveyed individuals from the Terry Collection, specimens identified to have died of causes other than TB could still have suffered from TB at death. Thus, in the aforementioned cases with PAs, APDIs or ABVIs in the NTB group, the tuberculous origin of the recorded endocranial and non-endocranial lesions cannot be excluded. However, it is also possible that in these cases - similar to the other one-half of individuals with PAs, APDIs or ABVIs in the NTB group, 
where no signs of other probable TBM-related endocranial alterations or of likely TBassociated non-endocranial bony changes were observed -, medical conditions other than TB might result in the development of the detected lesions.

Among the four evaluated endocranial alteration types, GIs may have the strongest diagnostic value, as they occurred about ten times more often in the TB group than in the NTB group; and - similar to the APDIs and ABVIs - the $\chi^{2}$ comparison of the frequencies of GIs revealed a statistically extremely significant difference between GIs and TBM; therefore, suggesting a strong correlation between GIs and TBM. Furthermore, the results of the current $\mathrm{PhD}$ dissertation may support those of previous studies regarding the specificity of GIs for TBM, as - in contrast to the APDIs, PAs, and ABVIs - GIs affected only the minority of the skeletons composing the NTB group, and in the vast majority of the abovementioned cases, probable TBM-associated endocranial alterations other than GIs and/or possible TB-related non-endocranial bony changes were also recorded. It must be noted that even if the registered cause of death of the surveyed individuals may not have been TB, specimens could still have suffered from the disease but their death was attributed to another medical condition; thus, it is very likely that in the above-mentioned cases, the observed endocranial and non-endocranial bony changes might result from TB.

In summary, even if PAs, APDIs, and ABVIs cannot be considered as pathognomonic features of TBM, palaeopathologists could still use them - with necessary circumspection - to identify the disease in osteoarchaeological material from the preantibiotic era, especially when they simultaneously occur with each other and/or with possible TB-related non-endocranial bony changes. Moreover, the findings of the present $\mathrm{PhD}$ dissertation may confirm that GIs can be considered as specific vestiges of TBM; and therefore, the palaeopathological diagnosis of TBM can be established with a high certainty when GIs are present in ancient human bone remains.

Finally, the results of the current $\mathrm{PhD}$ dissertation may provide palaeopathologists with a stronger basis for identifying $\mathrm{TB}$ in osteoarchaeological material from the preantibiotic era and with a more sensitive means of assessing the prevalence of the disease in past human populations. Nevertheless, further investigations on human skeletons of known cause of death from documented collections other than the Terry Collection are necessary to confirm the trends noted in the present research project. Refinement of macromorphological diagnostic criteria and their application in the palaeopathological practice may open new perspectives also in the research regarding the evolution of tuberculosis. 
9 ÖSSZEFOGLALÓ 
A tuberkulózis (tbc) az egyik legősibb fertőző megbetegedés, amely már évezredek óta sújtja az emberiséget és az állatvilágot. A paleopatológusok retrospektív módon, a modern orvosi szakirodalom alapján kísérelik meg a tbc diagnosztizálását a különbözö régészeti korokból származó történeti népességekben azáltal, hogy a betegséggel összefüggésbe hozható csontléziókat azonosítanak az egykor élt emberek maradványain. Ugyanakkor a tbc modern diagnosztikai kritériumai nem feltétlenül alkalmazhatók a paleopatológiai gyakorlatban, mivel a recens esetekben megfigyelhető tbc-s eredetü csontelváltozások különbözhetnek a történeti embertani leleteken található lézióktól, többek között azért, mert antituberkulotikus szereket is használnak a tbc kezelésében, amelyek befolyásolhatják a betegség manifesztációját. Emellett a modern klinikai esetekben a csontok nem vizsgálhatók közvetlenül makroszkópos módszerekkel, csak orvosi képalkotó eljárásokkal, amelyek segítségével azonban a nagyon enyhe, kisméretű csontelváltozások sok esetben nem észlelhetők. Ennek következtében a tbc-hez köthető minor csontléziók nem feltétlenül kerülnek említésre a modern orvosi szakirodalomban, mivel nem relevánsak a betegség diagnózisának felállításában.

Mindazonáltal, a preantibiotikus érából származó, ismert halálozási okú egyének csontvázaiból álló, jól dokumentált oszteológiai gyüjtemények felbecsülhetetlen értékkel bírnak a különböző betegségek, így a tbc paleopatológiai gyakorlatban is alkalmazható diagnosztikai kritériumainak meghatározásában, mivel a tbc-ben elhalálozott és antituberkulotikus szerekkel nem kezelt egyének maradványain olyan, a tbc-vel összefüggésbe hozható csontelváltozások azonosíthatók, amelyek a történeti népességekben is megfigyelhetök. Emellett a fent említett gyüjteményekben örzött csontvázleletek közvetlenül makroszkópos módszerekkel is vizsgálhatók, és a nagyon enyhe, kisméretü csontelváltozások is észlelhetők rajtuk. A múlt század vége óta számos, történeti embertani szériákon és dokumentált oszteológiai gyüjteményeken végzett paleopatológiai és paleomikrobiológiai tanulmány összefüggésbe hozta a tbc-s agyhártyagyulladást különböző, a koponya endocranialis felszínén megfigyelhető csontléziókkal, így a fokozott gödörkézettséggel (abnormally pronounced digital impression = APDI), a periostealis újcsontképződményekkel (periosteal apposition $=\mathrm{PA}$ ), a rendellenes érbenyomatokkal (abnormal blood vessel impression $=\mathrm{ABVI})$, valamint a granularis benyomatokkal (granular impression $=\mathrm{GI})$. Azonban az említett endocranialis elváltozások diagnosztikai értékét a közelmúltban többen megkérdőjelezték, mivel a pontos etiológiájuk jelenleg nem ismert, és a tbc-s meningitis mellett több más megbetegedés is felelős lehet a kialakulásukért. 
PhD értekezésem fó célja a paleopatológiai szakirodalomban korábban tbc-s agyhártyagyulladással összefüggésbe hozott endocranialis elváltozásokkal, valamint azok kialakulásával kapcsolatos ismereteink bővítése, továbbá a fent említett léziók mint a tbc paleopatológiai diagnosztizálása során alkalmazható kritériumok diagnosztikai értékének megerősítése.

Dolgozatom első fele a tbc-vel kapcsolatos modern orvosi és paleopatológiai szakirodalmat tekinti át, különös tekintettel a betegséghez köthető csontelváltozásokra. Az értekezés második része a Robert J. Terry Anatomical Skeletal Collection csontvázain végzett makromorfológiai és statisztikai elemzések eredményeit mutatja be, amelyek - a fent említett dokumentált oszteológiai gyüjteményen végzett paleopatológiai vizsgálatok közül elsőként - a tbc-s meningitis-hez köthető négyféle endocranialis léziótípus makromorfológiai jellegzetességeire, gyakoriságára, együttes előfordulására, valamint a tbcvel összefüggésbe hozható nem-endocranialis elváltozásokkal való asszociációjára fókuszáltak. A vizsgálatok során a Terry Collectionben található 302 tbc-ben elhalálozott egyén csontváza (tbc-s csoport) mellett 302 véletlenszerüen kiválasztott, nem tbc-ben elhalálozott egyén csontvázát (nem tbc-s csoport) is kiértékeltem.

\section{PhD értekezésem célkitűzései a következők:}

1) A Terry Collection kiválasztott csontvázainak makromorfológiai elemzése a négyféle tbc-s agyhártyagyulladáshoz köthető endocranialis léziótípus, valamint azok együttes megjelenése és a tbc-vel összefüggésbe hozható nem-endocranialis elváltozásokkal való asszociációja szempontjából;

2) A négyféle vizsgált endocranialis léziótípus, valamint azok együttes megjelenése és a tbchez köthető nem-endocranialis elváltozásokkal való asszociációja gyakoriságának összehasonlítása a tbc-s és nem tbc-s csoportban, a kiválasztott egyének nemének és elhalálozási életkorának figyelembevételével;

3) A négyféle vizsgált endocranialis léziótípus makromorfológiai jellegzetességeinek (fokozott gödörkézettség: kifejezettség; periostealis újcsontképződmények, rendellenes érbenyomatok és granularis benyomatok: elhelyezkedés, kiterjedtség és léziók száma az érintett koponyacsontokon) kiértékelése;

4) A négyféle vizsgált endocranialis léziótípus legfontosabb makromorfológiai jellegzetességeit mutató, a Terry Collectionből származó példaesetek bemutatása; és

5) A négyféle vizsgált endocranialis léziótípus mint a tbc paleopatológiai diagnosztizálásában alkalmazható kritériumok diagnosztikai értékének elemzése. 


\section{PhD dolgozatom eredményei a következő fö pontokban foglalhatók össze:}

1) A négyféle tbc-hez köthető endocranialis léziótípus közül legalább egyféle jelenlétét a Terry Collectionben vizsgált csontvázak mintegy kétharmadánál figyeltem meg. A leggyakoribb elváltozástípusnak a fokozott gödörkézettség (APDI) bizonyult. Periostealis újcsontképződményeket (PA), rendellenes érbenyomatokat (ABVI) és granularis benyomatokat (GI) a fokozott gödörkézettséghez képest kisebb, de egymáshoz hasonló arányban jegyeztem fel. A négyféle vizsgált endocranialis léziótípus együttes megjelenését, valamint a tbc-hez köthető nem-endocranialis elváltozásokkal való asszociációját a csontvázak mintegy egyharmadánál írtam le.

2) Mind a négy vizsgált endocranialis léziótípus előfordult a tbc-s és a nem tbc-s csoportban is, ugyanakkor valamennyi elváltozástípus gyakoribb volt a tbc-ben elhalálozott egyének körében: az APDI és a PA mintegy kétszer, az ABVI három és félszer, a GI pedig csaknem tízszer gyakrabban fordult elő a tbc-s csoportban, mint a nem tbc-s csoportban. Emellett a négyféle vizsgált endocranialis léziótípus együttes megjelenése és a tbc-hez köthető nem-endocranialis elváltozásokkal való asszociációja is gyakoribb volt a tbc-ben elhalálozott egyének körében: míg az előbbi mintegy ötször, az utóbbi mintegy háromszor gyakrabban fordult elő a tbc-s csoportban, mint a nem tbc-s csoportban. A $\chi^{2}$-próba alapján a két vizsgálati csoport közötti különbség mind a négy vizsgált endocranialis léziótípus, valamint azok együttes előfordulása és a tbc-hez köthető nem-endocranialis elváltozásokkal való asszociációja tekintetében is szignifikáns volt, ami - a korábbi tanulmányok eredményeihez hasonlóan - arra utal, hogy van összefüggés az APDI, a PA, az ABVI, valamint a GI és a tbc között, így a fent említett elváltozások valóban alkalmazhatók diagnosztikai kritériumokként a tbc paleopatológiai diagnosztizálása során.

3) A négyféle vizsgált endocranialis léziótípus makromorfológiai jellegzetességeit tekintve elmondható, hogy a Terry Collectionben vizsgált egyének maradványain feljegyzett APDI kifejezettsége az esetek mintegy kétharmadában nagyon enyhe volt. A PA, az ABVI és a GI lokalizációjára vonatkozó megfigyeléseim alátámasztják a témában megjelent korábbi publikációk eredményeit: a PA és az ABVI a homlokcsonton és a falcsontokon, a GI pedig a nyakszirtcsonton és a halántékcsontokon fordult elő leggyakrabban, és az utóbbi léziótípus eloszlási mintázata nagyfokú hasonlóságot mutatott a tbc-s meningitis patogenezise során az agyhártyákon kialakuló tbc-s gümők eloszlási mintázatával. Míg a megfigyelt PA-k és ABVI-k többsége az érintett koponyacsontok endocranialis felszínének kevesebb, mint felét borította, addig a leírt GI-k által borított terület kiterjedtsége csak ritkán 
lépte túl az érintett koponyacsontok endocranialis felszínének egynegyedét. A Terry Collection kiválasztott csontvázain talált PA-k, ABVI-k és GI-k többségét multifokális lézióként jegyeztem fel.

4) Doktori értekezésemben számos, a fokozott gödörkézettség (APDI), a periostealis újcsontképződmények (PA), a rendellenes érbenyomatok (ABVI) és a granularis benyomatok (GI) legfontosabb makromorfológiai jellegzetességeit mutató, a Terry Collectionből származó példaesetet ismertettem. A példák révén nagyobb betekintést nyerhetünk a négyféle vizsgált endocranialis léziótípus makromorfológiai megjelenési formáiba, emellett nagyobb megbízhatósággal és pontossággal állíthatjuk fel a tbc-s meningitis diagnózisát olyan történeti embertani leleteknél, amelyek a példaesetekben megfigyeltekhez hasonló endocranialis elváltozásokat mutatnak.

5) A Terry Collection kiválasztott csontvázain végzett makromorfológiai és statisztikai elemzések eredményei alapján mind a négy vizsgált endocranialis léziótípus alkalmazható diagnosztikai kritériumként a tbc-s agyhártyagyulladás paleopatológiai vizsgálata során, ugyanakkor az egyes elváltozástípusok diagnosztikai értéke különböző.

A legkisebb diagnosztikai értékkel a PA rendelkezik. Bár mintegy kétszeres gyakorisággal fordult elő a tbc-ben elhalálozott egyének körében, a $\chi^{2}$-próba alapján a PA és a tbc közötti összefüggés gyengébb, mint a másik három endocranialis léziótípus esetén. Az APDI annak ellenére, hogy a PA-hoz hasonlóan mintegy kétszer gyakrabban fordult elő a tbc-s csoportban, mint a nem tbc-s csoportban, a $\chi^{2}$-próba alapján erösebb összefüggést mutat a tbc-vel, mint a PA. A PA-hoz és az APDI-hoz viszonyítva az ABVI még erősebb diagnosztikai értékkel rendelkezik, mivel három és félszer gyakrabban fordult elö a tbc-ben elhalálozott egyének körében, és a $\chi^{2}$-próba alapján az APDI-hoz hasonlóan erős összefüggés van a lézió előfordulása és a tbc között.

Mind a három endocranialis léziótípus előfordult a nem tbc-s csoportba tartozó egyének körében is (PA: $\sim 10 \%$, APDI: $\sim 30 \%$, ABVI: $\sim 6 \%$ ), ugyanakkor ezen esetek mintegy felében a PA, az APDI vagy az ABVI mellett más tbc-vel összefüggésbe hozható endocranialis elváltozástípust és/vagy nem-endocranialis léziót is megfigyeltem a maradványokon. A Terry Collection részét képező egyének boncolási jegyzőkönyvén és/vagy halotti bizonyítványán szereplő halálozási ok mellett az egyének más megbetegedésekben, így tbc-ben is szenvedhettek a haláluk idején, emiatt a fent említett esetekben a megfigyelt endocranialis és nem-endocranialis léziók tbc-s eredete sem zárható ki. Azonban az is lehetséges, hogy - azokhoz az esetekhez hasonlóan, ahol a PA-n, APDI-n vagy ABVI-n kívül semmilyen más tbc-vel összefüggésbe hozható endocranialis léziótípust 
vagy nem-endocranialis elváltozást sem találtam - nem a tbc, hanem más megbetegedések álltak a leírt PA-k, APDI-k vagy ABVI-k kialakulása hátterében.

A négyféle vizsgált endocranialis léziótípus közül eredményeim alapján a GI bizonyult a legerősebb diagnosztikai értékünek, mivel ez az elváltozástípus mintegy tízszer gyakrabban fordult elő a tbc-ben elhalálozott egyének körében, és az APDI-hoz és az ABVIhoz hasonlóan a $\chi^{2}$-próba is erős összefüggést mutatott a GI és a tbc között. Továbbá a PAval, az APDI-val és az ABVI-val ellentétben a GI a nem tbc-ben elhalálozott egyének esetében csak elenyésző számban fordult elö, és egy eset kivételével a GI-n kívül más tbchez köthető endocranialis léziótípust és/vagy nem-endocranialis elváltozást is megfigyeltem a csontvázakon. Fontos megjegyezni, hogy a nem tbc-s csoportba tartozó egyének - annak ellenére, hogy boncolási jegyzőkönyvükön és/vagy halotti bizonyítványukon nem a tbc-t jelölték meg elhalálozási okként - a halálukat okozó megbetegedés mellett tbc-ben is szenvedhettek. Ebből kifolyólag a fent említett esetekben a megfigyelt endocranialis és nemendocranialis léziók nagy valószínűséggel tbc-s eredetre vezethetők vissza.

Összegzésként elmondható, hogy a periostealis újcsontképződmények (PA), a fokozott gödörkézettség (APDI) és a rendellenes érbenyomatok (ABVI) nem tekinthetők patognomikus tbc-s csontelváltozásnak. Azonban megfelelő körültekintéssel mindhárom endocranialis léziótípus alkalmazható diagnosztikai kritériumként a tbc paleopatológiai diagnosztizálása során, különösen azokban az esetekben, ahol egymással és/vagy tbc-hez köthető nem-endocranialis elváltozásokkal együtt fordulnak elö a történeti embertani leleteken. A granularis benyomatok (GI) tbc-specifikusságára vonatkozóan értekezésem eredményei fontos bizonyítékot szolgáltatnak. A Terry Collection ismert halálozási okú leletein végzett vizsgálatok tanúsága alapján azokban az esetekben, ahol GI figyelhető meg a történeti embertani leleteken, nagy biztonsággal állítható fel a tbc-s meningitis paleopatológiai diagnózisa.

Végezetül, $\mathrm{PhD}$ értekezésem eredményei szilárdabb alapot biztosítanak a paleopatológusok számára a tbc diagnosztizálásában és a betegség prevalenciájának becslésében a különböző régészeti korokból származó történeti népességekben. Mindazonáltal, a jövőben további, a preantibiotikus érából származó, ismert halálozási okú egyének csontvázaiból álló, jól dokumentált oszteológiai gyűjtemények vizsgálatára is szükség van ahhoz, hogy megerősítést nyerjenek a Terry Collectionben végzett makromorfológiai és statisztikai elemzések során kirajzolódott trendek. A makromorfológiai diagnosztikai kritériumok finomítása és paleopatológiai gyakorlatban való alkalmazása új perspektívákat nyithat meg a tbc evolúciójának a kutatásában is. 
10 REFERENCES 
1) Acharya J, Gibbs WN. 2016. Imaging spinal infection. Radiology of Infectious Diseases 3(2): 84-91. DOI: $10.1016 /$ j.jrid.2016.03.001

2) Agrawal V, Patgaonkar PR, Nagariya SP. 2010. Tuberculosis of spine. Journal of Craniovertebral Junction and Spine 1(2): 74-85. DOI: 10.4103/0974-8237.77671

3) Al-Khudaira N, Meir A. 2014. Isolated tuberculosis of the posterior spinal elements: case report and discussion of management. Journal of the Royal Society of Medicine Open 5(9): 1-6. DOI: $10.1177 / 2054270414543396$

4) Allison MJ, Mendoza D, Pezzia A. 1973. Documentation of a case of tuberculosis in pre-Columbian America. American Review of Respiratory Disease 107(6): 985-991. DOI: 10.1164/arrd.1973.107.6.985

5) Al-Sayyad MJ, Abumunaser LA. 2011. Tuberculous arthritis revisited as a forgotten cause of monoarticular arthritis. Annals of Saudi Medicine 31(4): 398-401. DOI: 10.4103/0256-4947.83210

6) Anselmo D, Capela e Silva F, Fernandes T. 2016. Characterization of hypertrophic osteoarthropathy in an identified skeleton from Évora, Portugal, using combined and comparative morphology and microscopy. International Journal of Paleopathology 12: 11-16. DOI: 10.1016/j.ijpp.2015.11.001

7) Arbeláez A, Medina E, Restrepo F, Castillo M. 2004. Cerebral tuberculosis. Seminars in Roentgenology 39(4): 474-481. DOI: 10.1053/j.ro.2004.06.003

8) Arriaza BT, Salo W, Aufderheide AC, Holcomb TA. 1995. Pre-Columbian tuberculosis in Northern Chile: Molecular and skeletal evidence. American Journal of Physical Anthropology 98(1):37-45. DOI: 10.1002/ajpa.1330980104

9) Arrieta MA, Bordach MA, Mendonça OJ. 2014. Pre-Columbian tuberculosis in northwest Argentina: Skeletal evidence from Rincón Chico 21 cemetery. International Journal of Osteoarchaeology 24(1): 114. DOI: $10.1002 /$ oa. 1300

10) Aschoff L. 1936. Tuberkulose der Hirn- und Rückenmarkshäute. In Pathologische Anatomie. Ein Lehrbuch für Studierende und Ärzte. Zweiter Band: Spezielle pathologische Anatomie. Gustav Fischer Verlag: Jena, Germany; 386-387.

11) Assis S, Santos AL, Roberts CA. 2011. Evidence of hypertrophic osteoarthropathy in individuals from the Coimbra Skeletal Identified Collection (Portugal). International Journal of Paleopathology 1(3-4): 155-163. DOI: 10.1016/j.ijpp.2012.02.009

12) Aufderheide AC, Rodríguez-Martín C. 1998. The Cambridge encyclopedia of human paleopathology. Cambridge University Press: Cambridge, UK; 118-141.

B

13) Baker BJ. 1999. Early manifestations of tuberculosis in the skeleton. In Tuberculosis: past and present, Pálfi Gy, Dutour O, Deák J, Hutás I (eds). TB Foundation: Szeged, Hungary \& Golden Book Publisher: Budapest, Hungary; 301-307.

14) Baker O, Lee OY-C, Wu HHT, Besra GS, Minnikin DE, Llewellyn G, Williams CM, Maixner F, O’Sullivan N, Zink A, Chamel B, Khawam R, Coqueugniot E, Helmer D, Le Mort F, Perrin P, Gourichon 
L, Dutailly B, Pálfi Gy, Coqueugniot H, Dutour O. 2015. Human tuberculosis predates domestication in ancient Syria. Tuberculosis 95(Suppl. 1): S4-S12. DOI: 10.1016/j.tube.2015.02.001

15) Balázs J, Zádori PG, Vandulek Cs, Molnár E, Ősz B, Bereczki Zs, Paja L, Palkó A, Fogas O, Zink A, Nerlich A, Pálfi Gy. 2015. Morphological and paleoradiological studies of Pott's disease cases. Acta Biologica Szegediensis 59(2): 211-216.

16) Bano S, Chaudhary V, Yadav S. 2012. Central nervous system tuberculosis. In Neuroimaging - Clinical Applications, Bright P (ed). InTech: Rijeka, Croatia; 467-498. DOI: 10.5772/24964

17) Bañuls A-L, Sanou A, Nguyen TVA, Godreuil S. 2015. Mycobacterium tuberculosis: ecology and evolution of a human bacterium. Journal of Medical Microbiology 64(11): 1261-1269. DOI: 10.1099/jmm.0.000171

18) Bartels P. 1907. Tuberkulose (Wirbelkaries) in der Jüngeren Steinzeit. Archiv für Anthropologie 34: 243255.

19) Be NA, Kim KS, Bishai WR, Jain SK. 2009. Pathogenesis of central nervous system tuberculosis. Current Molecular Medicine 9(2): 94-99. DOI: 10.2174/156652409787581655

20) Bell WE. 1978. Increased intracranial pressure - diagnosis and management. Current Problems in Pediatrics 8(4): 1-62. DOI: 10.1016/S0045-9380(78)80005-X

21) Bennike P. 1999. Facts or myths? A re-evaluation of cases of diagnosed tuberculosis in the past in Denmark. In Tuberculosis: past and present, Pálfi Gy, Dutour O, Deák J, Hutás I (eds). TB Foundation: Szeged, Hungary \& Golden Book Publisher: Budapest, Hungary; 509-518.

22) Bernaerts A, Vanhoenacker FM, Parizel PM, Van Goethem JW, Van Altena R, Laridon A, De Roeck J, Coeman V, De Schepper AM. 2003. Tuberculosis of the central nervous system: overview of neuroradiological findings. European Radiology 13(8): 1876-1890. DOI: 10.1007/s00330-002-1608-7

23) Bill P. 2006. Tuberculous meningitis. Continuing Medical Education 24(9): 505-511.

24) Bini EI, Hernández Pando R. 2014. Pathogenesis and immune response in tuberculous meningitis. The Malaysian Journal of Medical Sciences 21(1): 4-10.

25) Bir SC, Patra DP, Maiti TK, Sun H, Guthikonda B, Notarianni C, Nanda A. 2016. Epidemiology of adultonset hydrocephalus: institutional experience with 2001 patients. Neurosurgical Focus 41(3): e5. DOI: 10.3171/2016.7.FOCUS16188

26) Blondiaux J, Hédain V, Chastanet P, Pavaut M, Moyart V, Flipo R-M. 1999. Epidemiology of tuberculosis: A $4^{\text {th }}$ to $12^{\text {th }} \mathrm{c}$. AD picture in a 2498-skeleton series from northern France. In Tuberculosis: past and present, Pálfi Gy, Dutour O, Deák J, Hutás I (eds). TB Foundation: Szeged, Hungary \& Golden Book Publisher: Budapest, Hungary; 519-530.

27) Bos KI, Harkins KM, Herbig A, Coscolla M, Weber N, Comas I, Forrest SA, Bryant JM, Harris SR, Schuenemann VJ, Campbell TJ, Majander K, Wilbur AK, Guichon RA, Wolfe Steadman DL, Collins Cook D, Niemann S, Behr MA, Zumarraga M, Bastida R, Huson D, Nieselt K, Young D, Parkhill J, Buikstra JE, Gagneux S, Stone AC, Krause J. 2014. Pre-Columbian mycobacterial genomes reveal seals as a source of New World human tuberculosis. Nature 514(7523): 494-497. DOI: 10.1038/nature13591 
28) du Boulay GH. 1956. The significance of digital impressions in children's skulls. Acta Radiologica 46(12): 112-122. DOI: 10.3109/00016925609170820

29) du Boulay GH. 1980. Raised intracranial pressure. In Principles of X-ray diagnosis of the skull. Butterwort: London, UK; 1-28.

30) Brancusi F, Farrar J, Heemskerk D. 2012. Tuberculous meningitis in adults: a review of a decade of developments focusing on prognostic factors for outcome. Future Microbiology 7(9): 1101-1116. DOI: $10.2217 / \mathrm{fmb} .12 .86$

31) Brisse S, Supply P, Brosch R, Vincent V, Gutierrez MC. 2006. "A re-evaluation of M. prototuberculosis": Continuing the debate. PLoS Pathogens 2(9): e95. DOI: 10.1371/journal.ppat.0020095

32) Brites D, Gagneux S. 2015. Co-evolution of Mycobacterium tuberculosis and Homo sapiens. Immunological Reviews 264(1): 6-24. DOI: 10.1111/imr.12264

33) Brodsky MC. 2010. The swollen optic disc in childhood. In Pediatric neuro-ophthalmology. Springer Science \& Business Media: Berlin, Germany; 97-154.

34) Brosch R, Gordon SV, Marmiesse M, Brodin P, Buchrieser C, Eiglmeier K, Garnier T, Gutierrez C, Hewinson G, Kremer Parsons LM, Pym AS, Samper S, van Soolingen D, ST Cole. 2002. A new evolutionary scenario for the Mycobacterium tuberculosis complex. Proceedings of the National Academy of Sciences of the United States of America 99(6): 3684-3689. DOI: 10.1073_pnas.052548299

35) Buzhilova A, Pálfi Gy, Dutour O. 1999. A medieval case of possible sacroiliac joint tuberculosis and its archaeological context. In Tuberculosis: past and present, Pálfi Gy, Dutour O, Deák J, Hutás I (eds). TB Foundation: Szeged, Hungary \& Golden Book Publisher: Budapest, Hungary; 323-329.

C

36) Chan JZ, Sergeant MJ, Lee OY-C, Minnikin DE, Besra GS, Pap I, Spigelman M, Donoghue HD, Pallen MJ. 2013. Metagenomic analysis of tuberculosis in a mummy. The New England Journal of Medicine 369(3): 289-290. DOI: 10.1056/NEJMc1302295

37) Chaudhary V, Bano S, Garga UC. 2017. Central nervous system tuberculosis: an imaging perspective. Canadian Association of Radiologists Journal 68(2): 161-170. DOI: 10.1016/j.carj.2016.10.007

38) Cherian A, Thomas SV. 2011. Central nervous system tuberculosis. African Health Sciences 11(1): 116127.

39) Christensen A-SH, Andersen $\AA$ B, Thomsen VØ, Andersen PH, Johansen IS. 2011. Tuberculous meningitis in Denmark: a review of 50 cases. BMC Infectious Diseases 11: 47. DOI: 10.1186/1471-2334$11-47$

40) Cieślik AI. 2017. Evidence of tuberculosis among children in medieval ( $13^{\text {th }}-15^{\text {th }}$ century) Wrocław: A case study of hip joint tuberculosis in a juvenile skeleton excavated from the crypt of the St. Elizabeth church. Anthropological Review 80(2): 219-231. DOI: 10.1515/anre-2017-0014

41) Clarke C, van Helden P, Miller M, Parsons S. 2016. Animal-adapted members of the Mycobacterium tuberculosis complex endemic to the southern African subregion. Journal of the South African Veterinary Association 87(1): a1322. DOI: 10.4102/jsava.v87i1.1322 
42) Colombo A, Saint-Pierre C, Naji S, Panuel M, Coqueugniot H, Dutour O. 2015. Langerhans cell histiocystosis or tuberculosis on a medieval child (Oppidum de la Granède, Millau, France $-10^{\text {th }}-11^{\text {th }}$ centuries AD). Tuberculosis 95(Suppl. 1): S42-S50. DOI: 10.1016/j.tube.2015.02.003

43) Comas I, Coscolla M, Luo T, Borrell S, Holt KE, Kato-Maeda M, Parkhill J, Malla B, Berg S, Thwaites G, Yeboah-Manu D, Bothamley G, Mei J, Wei L, Bentley S, Harris SR, Niemann S, Diel R, Aseffa A, Gao Q, Young D, Gagneux S. 2013. Out-of-Africa migration and Neolithic coexpansion of Mycobacterium tuberculosis with modern humans. Nature Genetics 45(10): 1176-1182. DOI: $10.1038 / \mathrm{ng} .2744$

44) Coscolla M, Gagneux S. 2014. Consequences of genomic diversity in Mycobacterium tuberculosis. Seminars in Immunology 26(6): 431-444. DOI: 10.1016/j.smim.2014.09.012

D

45) Daniele B. 2014. Characteristics of central nervous system tuberculosis in a low-incidence country: a series of 20 cases and a review of the literature. Japanase Journal of Infectious Diseases 67(1): 50-53. DOI: $10.7883 /$ yoken.67.50

46) David S, Mateus ARA, Duarte EL, Albuquerque J, Portugal C, Sancho L, Lavinha J, Gonçalves G. 2015. Determinants of the sympatric host-pathogen relationship in tuberculosis. PLoS One 10(11): e0140625. DOI: 10.1371/journal.pone.0140625

47) Dawson H, Brown KR. 2012. Childhood tuberculosis: A probable case from late medieval Somerset, England. International Journal of Paleopathology 2(1): 31-35. DOI: 10.1016/j.ijpp.2012.04.001

48) De la Garza Ramos R, Goodwin CR, Abu-Bonsrah N, Bydon A, Witham TF, Wolinsky J-P, Sciubba DM. 2017. The epidemiology of spinal tuberculosis in the United States: an analysis of 2002-2011 data. Journal of Neurosurgery: Spine 26(4): 507-512. DOI: 10.3171/2016.9.SPINE16174

49) Dendane T, Madani N, Zekraoui A, Belayachi J, Abidi K, Zeggwagh AA, Abouqal R. 2013. International Journal of Infectious Diseases 17(6): e461-e465. DOI: 10.1016/j.ijid.2013.01.026

50) Desai V, Priyadarshini SR, Sharma R. 2014. Copper beaten skull! Can it be a usual appearance?. International Journal of Clinical Pediatric Dentistry 7(1): 47-49. DOI: 10.5005/jp-journals-10005-1233

51) Donald PR, Schaaf HS, Schoeman JF. 2005. Tuberculous meningitis and miliary tuberculosis: The Rich focus revisited. Journal of Infection 50(3): 193-195. DOI: 10.1016/j.jinf.2004.02.010

52) Donoghue HD, Marcsik A, Matheson C, Vernon K, Nuorala E, Molto JE, Greenblatt CL, Spigelman M. 2005. Co-infection of Mycobacterium tuberculosis and Mycobacterium leprae in human archaeological samples: a possible explanation for the historical decline of leprosy. Proceedings of the Royal Society B 272(1561): 389-394. DOI: 10.1098/rspb.2004.2966

53) Donoghue HD, Spigelman M, O’Grady J, Szikossy I, Pap I, Lee OY-C, Wu HHT, Besra GS, Minnikin DE. 2015. Ancient DNa analysis - An established technique in charting the evolution of tuberculosis and leprosy. Tuberculosis 95(Suppl. 1): S140-S144. DOI: 10.1016/j.tube.2015.02.020

54) Dutour O, Pálfi Gy, Brun J-P, Bérato J, Panuel M, Haas CJ, Zink A, Nerlich AG. 1999. Morphological, paleoradiological and paleomicrobiological study of a French medieval case of tuberculous spondylitis 
with cold abscesses. In Tuberculosis: past and present, Pálfi Gy, Dutour O, Deák J, Hutás I (eds). TB Foundation: Szeged, Hungary \& Golden Book Publisher: Budapest, Hungary; 393-400.

$\mathbf{E}$

55) Ernst JD. 2012. The immunological life cycle of tuberculosis. Nature Reviews Immunology 12(8): 581591. DOI: $10.1038 /$ nri3259

56) Esteves S, Catarino I, Lopes D, Sousa C. 2017. Spinal tuberculosis: rethinking an old disease. Journal of Spine 6(1): 358. DOI: $10.4172 / 2165-7939.1000358$

$\mathbf{F}$

57) Flynn JL, Chan J. 2001. Tuberculosis: latency and reactivation. Infection and Immunity 69(7): 41954201. DOI: 10.1128/IAI.69.7.4195-4201.2001

58) Flynn JL, Chan J, Lin PL. 2011. Macrophages and control of granulomatous inflammation in tuberculosis. Mucosal Immunology 40(3): 271-278. DOI: 10.1038/mi.2011.14

59) Fogel N. 2015. Tuberculosis: a disease without boundaries. Tuberculosis 95(5): 527-531. DOI: 10.1016/j.tube.2015.05.017

60) Friend JAR, Watson JM. 1998. Tuberculosis. In Infectious diseases of the respiratory tract, Ellis ME (ed.). Cambridge University Press: Cambridge, UK; 244-267.

61) Fuentes Ferrer M, Gutiérrez Torres L, Ayala Ramírez O, Rumayor Zarzuelo M, del Prado González N. 2012. Tuberculosis of the spine. A systematic review of case series. International Orthopaedics 36(2): 221-231. DOI: 10.1007/s00264-011-1414-4

G

62) Gagneux S. 2012. Host-pathogen co-evolution in human tuberculosis. Philosophical Transactions of the Royal Society B 367(1590): 850-859. DOI: 10.1098/rstb.2011.0316

63) Galagan JE. 2014. Genomic insights into tuberculosis. Nature Reviews Genetics 15(5): 307-320. DOI: $10.1038 / \mathrm{nrg} 3664$

64) Garg RK. 1999. Tuberculosis of the central nervous system. Postgraduate Medical Journal 75(881): 133140. DOI: $10.1136 /$ pgmj. 75.881 .133

65) Garg RK. 2010. Tuberculous meningitis. Acta Neurologica Scandinavica 122(2): 75-90. DOI: 10.1111/j.1600-0404.2009.01316.x

66) Garg RK, Somvanshi DS. 2011. Spinal tuberculosis: a review. The Journal of Spinal Cord Medicine 34(5): 440-454. DOI: 10.1179/2045772311Y.0000000023

67) Gauba C, Varma M. 2005. Tuberculosis of the central nervous system. Apollo Medicine 2(1): 21-28. DOI: $10.1016 / \mathrm{S} 0976-0016(12) 60056-8$

68) Gengenbacher M, Kaufmann SHE. 2012. Mycobacterium tuberculosis: success through dormancy. FEMS Microbiology Reviews 36(3): 514-532. DOI: 10.1111/j.1574-6976.2012.00331.x

69) Getahun H, Matteelli A, Chaisson RE, Raviglione M. 2015. Latent Mycobacterium tuberculosis infection. New England Journal of Medicine 372(22): 2127-2135. DOI: 10.1056/NEJMra1405427 
70) Giacon M. 2008. Paleopathology in the osteological collection of Anthropological Museum in Padova University: the cases of tuberculosis. PhD Dissertation, Padova University: Padova, Italy.

71) Gideon HP, Flynn JL. 2011. Latent tuberculosis: what the host "sees"?. Immunology Research 50(2-3): 202-212. DOI: $10.1007 / \mathrm{s} 12026-011-8229-7$

72) Gładykowska-Rzeczycka J. 1999. Tuberculosis in the past and present in Poland. In Tuberculosis: past and present, Pálfi Gy, Dutour O, Deák J, Hutás I (eds). TB Foundation: Szeged, Hungary \& Golden Book Publisher: Budapest, Hungary; 559-573.

73) Golden MP, Vikram HR. 2005. Extrapulmonary tuberculosis: an overview. American Family Physician 72(9): 1761-1768.

74) Green JR. 1943. Chronic tuberculous meningo-encephalitis. Journal of Neuropathology and Experimental Neurology 2(2): 148-163. DOI: 10.1097/00005072-194304000-00005

75) Griffith JPC. 1919. The diseases of infants and children. W. B. Saunders Company: Philadelphia, USA \& London, UK.

76) Guichón RA, Buikstra JE, Stone AC, Harkins KM, Suby JA, Massone M, Iglesias AP, Wilbur A, Constantinescu F, Rodríguez Martín C. 2015. Pre-Columbian tuberculosis in Tierra del Fuego? Discussion of the paleopathological and molecular evidence. International Journal of Paleopathology 11: 92-101. DOI: 10.1016/j.ijpp.2015.09.003

77) Gupta R, Bienenstock H, Morano P, Gupta A. 2005. Tuberculosis of sacroiliac joint: an unusual presentation. Journal of the National Medical Association 97(8): 1174-1176.

78) Gutierrez MC, Brisse S, Brosch R, Fabre M, Omaïs B, Marmiesse M, Supply P, Vincent V. 2005. Ancient origin and gene mosaicism of the progenitor of Mycobacterium tuberculosis. PLoS Pathogens 1(1): e5. DOI: 10.1371/journal.ppat.0010005

H

79) Haas CJ, Zink A, Molnár E, Szeimies U, Reischl U, Marcsik A, Ardagna Y, Dutour O, Pálfi Gy, Nerlich AG. 2000. Molecular evidence for different stages of tuberculosis in ancient bone samples from Hungary. American Journal of Physical Anthropology 113(3): 293-304. DOI: 10.1002/10968644(200011)113:3<293::AID-AJPA2>3.0.CO;2-6

80) Hajdu T, Donoghue HD, Bernert Zs, Fóthi E, Kővári I, Marcsik A. 2012. A case of spinal tuberculosis from the Middle Ages in Transylvania (Romania). Spine 37(25): e1598-e1601. DOI: 10.1097/BRS.0b013e31827300dc

81) Held MFG, Hoppe S, Laubscher M, Mears S, Dix-Peek S, Zar HJ, Dunn RN. 2017. Epidemiology of musculoskeletal tuberculosis in an area with high disease prevalence. Asian Spine Journal 11(3): 405411. DOI: 10.4184/asj.2017.11.3.405

82) Hershberg R, Lipatov M, Small PM, Sheffer H, Niemann S, Homolka S, Roach JC, Kremer K, Petrov DA, Feldman MW, Gagneux S. 2008. High functional diversity in Mycobacterium tuberculosis driven by genetic drift and human demography. PLoS Biology 6(12): e311. DOI: 10.1371/journal.pbio.0060311

83) Hershkovitz I, Greenwald CM, Latimer B, Jellema LM, Wish-Baratz S, Eshed V, Dutour O, Rotschild BM. 2002. Serpens endocrania symmetrica (SES): a new term and a possible clue for identifying 
intrathoracic disease in skeletal populations. American Journal of Physical Anthropology 118(3): 201216. DOI: 10.1002/ajpa.10077

84) Hershkovitz I, Donoghue HD, Minnikin DE, May H, Lee OY-C, Feldman M, Galili E, Spigelman M, Rothschild BM, Bar-Gal GK. 2015. Tuberculosis origin: The Neolithic scenario. Tuberculosis 95(Suppl. 1): S122-S126. DOI: 10.1016/j.tube.2015.02.02

85) Horáčková L, Vargová L, Horváth R, Bartoš M. 1999. Morphological, roentgenological and molecular analyses in bone specimens attributed to tuberculosis, Moravia (Czeh Republic). In Tuberculosis: past and present, Pálfi Gy, Dutour O, Deák J, Hutás I (eds). TB Foundation: Szeged, Hungary \& Golden Book Publisher: Budapest, Hungary; 411-417.

86) Houshian S, Poulsen S, Riegels-Nielsen P. 2000. Bone and joint tuberculosis in Denmark. Acta Orthopaedica Scandinavica 71(3): 312-315. DOI: 10.1080/000164700317411942

87) Huard RC, Fabre M, de Haas P, Lazzarini LC, van Soolingen D, Cousins D, Ho JL. 2006. Novel genetic polymorphisms that further delineate the phylogeny of the Mycobacterium tuberculosis complex. Journal of Bacteriology 188(12): 4271-4287. DOI: 10.1128/JB.01783-05

88) Hunt DR, Albanese J. 2005. History and demographic composition of the Robert J. Terry Anatomical Collection. American Journal of Physical Anthropology 127(4): 406-417. DOI: 10.1002/ajpa.20135

89) Hutás I. 1999. The history of tuberculosis in Hungary. In Tuberculosis: past and present, Pálfi Gy, Dutour O, Deák J, Hutás I (eds). TB Foundation: Szeged, Hungary \& Golden Book Publisher: Budapest, Hungary; $37-42$.

I

90) Ingole K, Pawar S, Pathak S, Sharma A. 2016. Psoas abscess secondary to Pott's spine: a case report. IOSR Journal of Dental and Medical Sciences 15(5): 118-121. DOI: 10.9790/0853-150508118121

\section{J}

91) Jain AK. 2010. Tuberculosis of the spine: a fresh look at an old disease. The Journal of Bone and Joint Surgery (British Volume) 92(7): 905-913. DOI: 10.1302/0301-620X.92B7.24668

92) Jankauskas R. 1999. Tuberculosis in Lithuania: paleopathological and historical correlations. In Tuberculosis: past and present, Pálfi Gy, Dutour O, Deák J, Hutás I (eds). TB Foundation: Szeged, Hungary \& Golden Book Publisher: Budapest, Hungary; 549-5558.

93) Jankauskas R, Schultz M. 1995. Meningeal reactions in late medieval - early modern child population from Alytus, Lithuania. Journal of Paleopathology 7(2): 106.

94) Janovic A, Milovanovic P, Sopta J, Rakocevic Z, Filipovic V, Nenezic D, Djuric M. 2015. Intracranial arteriovenous malformations as a possible cause of endocranial bone lesions and associated neurological disorder. International Journal of Osteoarchaeology 25(1): 88-97. DOI: 10.1002/oa.2266

95) Jutte PC, van Loenhaut-Rooyackers JH, Borgdorff MW, van Horn JR. 2004. Increase of bone and joint tuberculosis in the Netherlands. The Journal of Bone and Joint Surgery (British Volume) 86(6): 901-904. DOI: 10.1302/0301-620X.86B6.14844 
96) Kajdocsi Lovász G. 2015. A török hódoltság kori idegen etnikumok összehasonlító embertani vizsgálata. Ph.D. Thesis, University of Szeged, Faculty of Science and Informatics, Szeged, Hungary.

97) Kam WL, Leung YF, Chung OM, Wai YL. 2000. Tuberculous osteomyelitis of the scapula. International Orthopaedics 24(5): 301-302. DOI: 10.1007/s002640000165

98) Katti MK. 2004. Pathogenesis, diagnosis, treatment, and outcome aspects of cerebral tuberculosis. Medical Science Monitor 10(9): RA215-229.

99) Kelley MA, Micozzi MS. 1984. Rib lesions in chronic pulmonary tuberculosis. American Journal of Physical Anthropology 65(4): 381-386. DOI: 10.1002/ajpa.1330650407

100) Kelly P, Manning P, Corcoran P, Clancy L. 1991. Hypertrophic osteoarthropathy in association with pulmonary tuberculosis. Chest 99(3): 769-770. DOI: 10.1378/chest.99.3.769

101) Kent SJ, Crowe SM, Yung A, Lucas CR, Mijch AM. 1993. Tuberculous meningitis: a 30-year review. Clinical Infectious Diseases 17(6): 987-994. DOI: 10.1093/clinids/17.6.987

102) Khoo JLS, Lau KY, Cheung CM, Tsoi TH. 2003. Central nervous system tuberculosis. Journal of Hong Kong College Radiologists 6: 217-228.

103) Köhler K, Pálfi Gy, Molnár E, Zalai-Gaál I, Osztás A, Bánffy E, Kirinó K, Kiss KK, Mende BG. 2014. A late Neolithic case of Pott's disease from Hungary. International Journal of Osteoarchaeology 24(6): 697-703. DOI: 10.1002/oa.2254

104) Köhler K, Voicsek V, Jakucs J, Pap I. 2016. Súlyos, fertőzéses eredetű csontelváltozás előfordulása a Kr. e. 6. évezred utolsó harmadában Versend-Gilencsa lelöhelyén. Anthropologiai Közlemények 57: 3-16. DOI: 10.20330/AnthropKozl.2016.57.3

105) Kumar R. 2005. Spinal tuberculosis: with reference to the children in northern India. Child's Nervous System 21(1): 19-26. DOI: 10.1007/s00381-004-1029-9

106) Kumar K. 2017. Posterior spinal tuberculosis: a review. Mycobacterial Diseases 7(2): 243. DOI: 10.4172/2161-1068.1000243

\section{$\mathbf{L}$}

107) Lagier R. 1999. Paleopathological diagnosis of skeletal tuberculosis. In Tuberculosis: past and present, Pálfi Gy, Dutour O, Deák J, Hutás I (eds). TB Foundation: Szeged, Hungary \& Golden Book Publisher: Budapest, Hungary; 283-290.

108) Lal H, Jain VK, Kannan S. 2013. Tuberculosis of the pubic symphysis: four unusual cases and literature review. Clinical Orthopaedics and Related Research 471(10): 3372-3380. DOI: 10.1007/s11999-0133037-0

109) Lewis ME. 2004. Endocranial lesions in non-adult skeletons: understanding their aetiology. International Journal of Osteoarchaeology 14(2): 82-97. DOI: 10.1002/oa.713

110) Lombardi GP, Cáceres UG. 2000. Multisystemic tuberculosis in a pre-Columbian Peruvian mummy: Four diagnostic levels, and a paleoepidemiological hypothesis. Chungara: Revista de Antropología Chilena 32(1): 55-60. 
111) Maczel M. 2003. „On the traces of tuberculosis” Diagnostic criteria of tuberculosis affection of the human skeleton and their application in Hungarian and French anthropological series. Ph.D. Thesis, University of La Méditerranée - Aix Marseille II Faculty of Medicine, Marseille, France \& University of Szeged, Faculty of Science, Szeged, Hungary.

112) Madkour MM. 2011a. Tuberculosis in ancient Egypt. In Tuberculosis. Springer Science \& Business Media, Berlin, Germany; 3-13.

113) Madkour MM. 2011b. Extraspinal musculoskeletal tuberculosis. In Tuberculosis. Springer Science \& Business Media, Berlin, Germany; 587-604.

114) Mahomed N, Sewchuran T, Mahomed Z. 2012. The copper-beaten skull. South African Journal of Radiology 16(1): 25-26. DOI: 10.4102/sajr.v16i1.229

115) Mansukhani S, Arora M, Kale S, Dhar S. 2014. An unusual case of atlanto-axial and basi-occipital TB with prevertebral abscess and epidural abscess. Journal of Rheumatology and Orthopedics 1(1): 4. DOI: $10.7243 / 2055-7000-1-4$

116) Marcsik A. 1978. Comparative evaluation of pathological Avar findings from excavations between the Danube and Tisza rivers. Acta Biologica Szegediensis 24(1-4): 143-150.

117) Marcsik A, Szentgyörgyi R, Gyetvai A, Finnegan M, Pálfi Gy. 1999. Probable Pott's paraplegia from the $7^{\text {th }} 8^{\text {th }}$ century AD. In Tuberculosis: past and present, Pálfi Gy, Dutour O, Deák J, Hutás I (eds.). TB Foundation: Szeged, Hungary \& Golden Book Publisher: Budapest, Hungary; 331-336.

118) Marcsik A, Molnár E, Ösz B, Donoghue HD, Zink A, Pálfi Gy. 2009. Adatok a lepra, tuberculosis és syphilis magyarországi paleopatológiájához. Folia Anthropologica 8: 5-34.

119) Mariotti V, Zuppello M, Pedrosi ME, Bettuzzi M, Brancaccio R, Peccenini E, Morigi MP, Belcastro MG. 2015. Skeletal evidence of tuberculosis in a modern identified human skeletal collection (Certosa cemetery, Bologna, Italy). American Journal of Physical Anthropology 157(3): 389-401. DOI: 10.1002/ajpa.22727

120) Marx GE, Chan ED. 2011. Tuberculous meningitis: diagnosis and treatment overview. Tuberculosis Research and Treatment DOI: 10.1155/2011/798764

121) Masson M, Molnár E, Donoghue HD, Besra GS, Minnikin DE, Wu HHT, Lee OY-C, Bull ID, Pálfi Gy. 2013. Osteological and biomolecular evidence of a 7000-year-old case of hypertrophic pulmonary osteopathy secondary to tuberculosis from Neolithic Hungary. PLoS ONE 8(10): e78252. DOI: 10.1371/journal.phone.0078252

122) Masson M, Bereczki Zs, Molnár E, Donoghue HD, Minnikin DE, Lee OY-C, Wu HHT, Besra GS, Bull ID, Pálfi Gy. 2015. 7000-year-old tuberculosis cases from Hungary - Osteological and biomolecular evidence. Tuberculosis 95(Suppl. 1): S13-S17. DOI: 10.1016/j.tube.2015.02.007

123) Matos V, Santos AL. 2006. On the trail of pulmonary tuberculosis based on rib lesions: results from the human identified skeletal collection from the Museu Bocage (Lisbon, Portugal). American Journal of Physical Anthropology 130(2): 190-200. DOI: 10.1002/ajpa.20309 
124) Meena S, Gangary SK. 2015. Tuberculosis of symphysis pubis: a case report. Journal of Research in Medical Sciences 20(1): 100-102.

125) Mensforth RP, Lovejoy CO, Lallo JW, Armelagos GJ. 1978. The role of constitutional factors, diet, and infectious disease in the etiology of porotic hyperostosis and periosteal reactions in prehistoric infants and children. Medical Anthropology 2(1): 1-59. DOI: 10.1080/01459740.1978.9986939

126) Ménard V. 1888. Tuberculose vertébrale. Asselin et Houzeau: Paris, France.

127) Minnikin DE, Lee OY-C, Wu HHT, Besra GS, Bhatt A, Nataraj V, Rothschild BM, Spigelman M, Donoghue HD. 2015. Ancient mycobacterial lipids: Key reference biomarkers in charting the evolution of tuberculosis. Tuberculosis 95(Suppl. 1): S133-S139. DOI: 10.1016/j.tube.2015.02.009

128) Miranda MS, Breiman A, Allain S, Deknuydt F, Altare F. 2012. The tuberculous granuloma: an unsuccessful host defence mechanism providing a safety shelter for the bacteria?. Clinical and Developmental Immunology DOI: 10.1155/2012/139127

129) Mohapatra D, Sarangi G, Paty BP, Das P, Mohapatra A, Chayani N, Patnaik G. 2011. Tuberculous synovitis of knee joint. Indian Medical Gazette 145(11): 457-459.

130) Molnár E, Donoghue HD, Lee OY-C, Wu HHT, Besra GS, Minnikin DE, Bull ID, Llewellyn G, Williams CM, Spekker O, Pálfi Gy. 2015. Morphological and biomolecular evidence for tuberculosis in $8^{\text {th }}$ century AD skeletons from Bélmegyer-Csömöki domb, Hungary. Tuberculosis 95(Suppl. 1): S35-S41. DOI: 10.1016/j.tube.2015.02.032

131) Morgado TC, Kinsky M, Carrara H, Rothemeyer S, Semple P. 2013. Prognostic value of computed topography-evident cerebral infarcts in adult patients with tuberculous meningitis and hydrocephalus treated with an external ventricular drain. World Neurosurgery 80(6): e255-e260. DOI: 10.1016/j.wneu.2012.09.021

132) Mostowy S, Cousins D, Brinkman J, Aranaz A, Behr MA. 2002. Genomic deletions suggest a phylogeny for the Mycobacterium tuberculosis complex. The Journal of Infectious Diseases 186(1): 74-80. DOI: $10.1086 / 341068$

133) Myers JN. 2007. Miliary, central nervous system, and genitourinary tuberculosis. Disease-a-Month 53(1): 22-31. DOI: 10.1016/j.disamonth.2006.10.003

N

134) Nerlich AG, Haas CJ, Zink A, Szeimies U, Hagedorn HG. 1997. Molecular evidence for tuberculosis in an ancient Egypt mummy. The Lancet 350(9088): 1404. DOI: 10.1016/S0140-6736(05)65185-9

135) Newton RW. 1994. Tuberculous meningitis. Archives of Disease in Childhood 70(5): 364-366. DOI: 10.1136/adc. 70.5 .364

136) Nicklisch N, Maixner F, Ganslmeier R, Friederich S, Dresely V, Meller H, Zink A, Alt KW. 2012. Rib lesions in skeletons from early Neolithic sites in central Germany: on the trail of tuberculosis at the onset of agriculture. American Journal of Physical Anthropology 149(3): 391-404. DOI: 10.1002/ajpa.22137

137) Nielsen N, Breedt A. 2013. Hydrocephalus. In Nursing care of the pediatric neurosurgery patient, Cartwright CC, Wallace DC (eds). Springer-Verlag: Berlin, Heidelberg, Germany; 37-84. DOI: 10.1007/978-3-642-32554-0_2 
138) Ortner DJ. 2003. Infectious diseases: tuberculosis and leprosy. In Identification of pathological conditions in human skeletal remains, Ortner DJ (ed.). Academic Press, San Diego, CA, USA; 227-271.

139) Ortner DJ. 2008. Differential diagnosis of skeletal lesions in infectious disease. In Advances in human paleopathology, Pinhasi R, Mays S (eds). John Wiley \& Sons, Ltd.: Chichester, UK; 191-214.

Ő

140) Ösz B, Hajnal K, Marcsik A, Fogas O, Horváth F, Zádori P, Kelemen K, Vandulek Cs, Schultz M, Márk L, Molnár E, Pálfi Gy. 2009. Preliminary report on the paleopathological research of the skeletal material from the Szeged medieval castle excavation. Acta Biologica Szegediensis 53(2): 125-138.

P

141) Pai M, Behr MA, Dowdy D, Dheda K, Divangahi M, Boehme CC, Ginsberg A, Swaminathan S, Spigelman M, Getahun H, Menzies D, Raviglione M. 2016. Tuberculosis. Nature Reviews Disease Primers 2: 16076. DOI: 10.1038/nrdp.2016.76

142) Paja L, Coqueugniot H, Dutour O, Willmon R, Farkas GyL, Palkó A, Pálfi Gy. 2015. Knee ankyloses associated with tuberculosis from the Medieval Hungary - Differential diagnosis based on medical imaging techniques. International Journal of Osteoarchaeology 25(3): 352-360. DOI: 10.1002/oa.2284

143) Palmer PES. 2002. Tuberculosis of bone. In The imaging of tuberculosis: with epidemiological, pathological, and clinical correlation. Springer Science \& Business Media, Berlin, Germany; 84-103.

144) Papagelopoulos PJ, Papadopoulos EC, Mavrogenis AF, Themistocleous GS, Korres DS, Soucacos PN. 2005. Tuberculous sacroiliitis. A case report and review of the literature. European Spine Journal 14(7): 683-688. DOI: 10.1007/s00586-004-0831-0

145) Patel R, Gannamani V, Shay E, Alcid D. 2016. Spinal tuberculosis and cold abscess without known primary disease: case report and review of the literature. Case Reports in Infectious Diseases DOI: $10.1155 / 2016 / 1780153$

146) Paul S, Smith J, Green J, Smith-Collins A, Chinthapalli R. 2013. Managing children with raised intracranial pressure: part one (introduction and meningitis). Nursing Children and Young People 25(10): 31-36. DOI: 10.7748/ncyp2013.12.25.10.31.e214

147) Paul S, Smith J, Green J, Smith-Collins A, Chinthapalli R. 2014. Managing children with raised intracranial pressure: part two (brain tumours and intracranial bleeds). Nursing Children and Young People 26(1): 30-37. DOI: 10.7748/ncyp2014.02.26.1.30.e214

148) Pálfi Gy, Dutour O, Deák J, Hutás I (eds). 1999. Tuberculosis: past and present. TB Foundation: Szeged, Hungary \& Golden Book Publisher: Budapest, Hungary.

149) Pálfi Gy, Bereczki Zs, Ortner DJ, Dutour O. 2012. Juvenile cases of skeletal tuberculosis from the Terry Anatomical Collection (Smithsonian Institution, Washington, DC, USA). Acta Biologica Szegediensis 56(1): $1-12$.

150) Pálfi Gy, Dutour O, Perrin P, Sola C, Zink A. 2015. Tuberculosis in evolution. Tuberculosis 95(Suppl. 1): S1-S3. DOI: 10.1016/j.tube.2015.04.007 
151) Pemmaiah DC. 2015. Copper beaten skull. Journal of Medical Sciences and Health 1(2): 46-48.

152) Pigrau-Serrallach C, Rodríguez-Pardo D. 2013. Bone and joint tuberculosis. European Spine Journal 22(Suppl. 4): S556-S566. DOI: 10.1007/s00586-012-2331-y

153) Pott P. 1779. Remarks on that kind of palsy of the lower limbs which is frequently found to accompany a curvature of the spine, and is supposed to be caused by it, together with its method of cure. To which are added, observations on the necessity and propriety of amputation, in certain cases, and under certain circumstances. J. Johnson: London, UK.

154) Pósa A, Maixner F, Sola C, Bereczki Zs, Molnár E, Masson M, Lovász G, Spekker O, Wicker E, Perrin P, Dutour O, Zink A, Pálfi Gy. 2015. Tuberculosis infection in a late-medieval Hungarian population. Tuberculosis 95(Suppl. 1): S60-S64. DOI: 10.1016/j.tube.2015.02.010

Q

155) Qureshi MA, Afzal W, Khalique AB, Pasha IF, Aebi M. 2013. Tuberculosis of the craniovertebral junction. European Spine Journal 22(Suppl. 4): S612-S617. DOI: 10.1007/s00586-012-2497-3

\section{R}

156) Rajashekar HK, Halesha BR, Chennaveerappa PK, Nagaral J, Dinakar VP. 2014. A study on the clinical features and complications of tuberculous meningitis in a tertiary care centre of Southern India. Journal of Evolution of Medical and Dental Sciences 3(34): 9031-9038. DOI: 10.14260/jemds/2014/3164

157) Rajshekhar V. 2009. Management of hydrocephalus in patients with tuberculous meningitis. Neurology India 57(4): 368-374. DOI: 10.4103/0028-3886.55572

158) Ramirez-Lapausa M, Menendez-Saldana A, Noguerado-Asensio A. 2015. Extrapulmonary tuberculosis: an overview. Revista Española de Sanidad Penitenciaria 17(1): 3-11. DOI: 10.4321/S157506202015000100002

159) Rasouli MR, Mirkoohi M, Vaccaro AR, Yarandi KK, Rahimi-Movaghar V. 2012. Spinal tuberculosis: diagnosis and management. Asian Spine Journal 6(4): 294-308. DOI: 10.4184/asj.2012.6.4.294

160) Raut T, Garg RK, Jain A, Verma R, Singh MK, Malhotra HS, Kohli N, Parihar A. 2013. Hydrocephalus in tuberculous meningitis: incidence, its predictive factors and impact on the prognosis. Journal of Infection 66(4): 330-337. DOI: 10.1016/j.jinf.2012.12.009

161) Reid R. 1949. Tuberculous glands of the neck. Postgraduate Medical Journal 25(288): 465-470. DOI: 10.1136/pgmj.25.288.465

162) Rich AR, McCordock HA. 1933. The pathogenesis of tuberculous meningitis. Bulletin of Johns Hopkins Hospital 52: 5-37. DOI: 10.1016/S0041-3879(34)80008-6

163) Rivas-Garcia A, Sarria-Estrada S, Torrents-Odin C, Casas-Gomila L, Franquet E. 2013. Imaging findings of Pott's disease. European Spine Journal 22(Suppl. 4): S567-S578. DOI: 10.1007/s00586-012-2333-9

164) Roberts CA, Buikstra JE. 2003. The bioarchaeology of tuberculosis: a global view on a reemerging disease. University Press of Florida: Gainesville, FL, USA.

165) Roberts CA, Lucy D, Manchester K. 1994. Inflammatory lesions of ribs: an analysis of the Terry Collection. American Journal of Physical Anthropology 95(2): 169-182. DOI: 10.1002/ajpa.1330950205 
166) Roberts CA, Pfister L-A, Mays S. 2009. Letter to the editor: Was tuberculosis present in Homo erectus in Turkey?. American Journal of Physical Anthropology 139(3): 442-444. DOI: 10.1002/ajpa.21056

167) Rock RB, Olin M, Baker CA, Molitor TW, Peterson PK. 2008. Central nervous system tuberculosis: pathogenesis and clinical aspects. Clinical Microbiology Reviews 21(2): 243-261. DOI: 10.1128/CMR.00042-07

168) Rothschild BM, Rothschild C. 1998. Recognition of hypertrophic osteoarthropathy in skeletal remains. The Journal of Rheumatology 25(11): 2221-2227.

169) Rothschild B, Rothschild C. 1999. Evolution of osseous/radiologic signs of tuberculosis. In Tuberculosis: past and present, Pálfi Gy, Dutour O, Deák J, Hutás I (eds). TB Foundation: Szeged, Hungary \& Golden Book Publisher: Budapest, Hungary; 291-298.

\section{S}

170) Sachdeva R, Sachdeva S, Arora S. 2013. Sternal tuberculosis. Annals of Medical and Health Sciences Research 3(Suppl. 1): S21-S23. DOI: 10.4103/2141-9248.121213

171) Saito H. 1956. Pathogenesis of tuberculous meningitis. Acta Tuberculosea Japonica 6(1):20-36.

172) Salekeen S, Mahmood K, Naqvi IH, Baig MY, Akhter ST, Abbasi A. 2013. Clinical course, complications and predictors of mortality in patients with tuberculous meningitis - an experience of fifty-two cases at the Civil Hospital, Karachi, Pakistan. Journal of Pakistan Medical Association 63(5): 563-567.

173) Salo WL, Aufderheide AC, Buikstra JE, Holcomb TA. 1994. Identification of Mycobacterium tuberculosis DNA in a pre-Columbian Peruvian mummy. Proceedings of the National Academy of Sciences of the United States of America 91(6): 2091-2094.

174) Sandher DS, Al-Jibury M, Paton RW, Ormerod LP. 2007. Bone and joint tuberculosis: cases in Blackburn between 1988 and 2005. The Journal of Bone and Joint Surgery (British Volume) 89(10): 1379-1381. DOI: 10.1302/0301-620X.89B10.18943

175) Santos AL, Roberts CA. 2001. A picture of tuberculosis in young Portuguese people in the early 20th century: a multidisciplinary study of the skeletal and historical evidence. American Journal of Physical Anthropology 115(1): 38-49. DOI: 10.1002/ajpa.1054

176) Santos AL, Roberts CA. 2006. Anatomy of a serial killer: differential diagnosis of tuberculosis based on rib lesions of adult individuals from the Coimbra Identified Skeletal Collection, Portugal. American Journal of Physical Anthropology 130(1): 38-49. DOI: 10.1002/ajpa.20160

177) Schmidt B. 1941. Chronische leptomeningitis cerebrospinalis tuberculosa. Lung 96(2): 124-138. DOI: 10.1007/BF02144352

178) Schmidt-Schultz TH, Schultz M. 2015. AG 85, a major secretion protein of Mycobacterium tuberculosis, can be identified in ancient bone. Tuberculosis 95(Suppl. 1): S87-S92. DOI: 10.1016/j.tube.2015.02.034

179) Schultz M. 1993. Spuren unspezifischer Entzündungen an prähistorischen und historischen Schädeln. Ein Beitrag zur Paläopathologie. Anthropologisches Forschungsinstitut: Aesch, Switzerland \& Anthropologische Gesellschaft: Basel, Switzerland. 
180) Schultz M. 1999. The role of tuberculosis in infancy and childhood in prehistoric and historic populations. In Tuberculosis: past and present, Pálfi Gy, Dutour O, Deák J, Hutás I (eds). TB Foundation: Szeged, Hungary \& Golden Book Publisher: Budapest, Hungary; 503-507.

181) Schultz M. 2001. Paleohistopathology of bone: a new approach to the study of ancient diseases. American Journal of Physical Anthropology 116(Suppl. 33): 106-147. DOI: 10.1002/ajpa.10024

182) Schultz M. 2003. Light microscopic analysis in skeletal paleopathology. In Identification of pathological conditions in human skeletal remains, Ortner DJ (ed.). Academic Press: San Diego, CA, USA; $73-107$. DOI: 10.1016/B978-012528628-2/50043-0

183) Schultz M, Schmidt-Schultz TH. 2015. Is it possible to diagnose TB in ancient bone using microscopy?. Tuberculosis 95(Suppl. 1): S80-S86. DOI: 10.1016/j.tube.2015.02.035

184) Schüller A. 1940-41. X-ray symptoms of intracranial hypertension. Confinia Neurologica 3(1-2): 253256. DOI: $10.1159 / 000106215$

185) Shapiro F. 2015. Bone and joint deformity in metabolic inflammatory neoplastic infectious and hematologic disorders. In Pediatric orthopedic deformities, volume 1: Pathobiology and treatmet of dysplasias, physeal fractures, length discrepancies, and epiphyseal and joint disorders. Springer: Berlin, Germany; 411-504.

186) Smith I. 2003. Mycobacterium tuberculosis pathogenesis and molecular determinants of virulence. Clinical Microbiology Reviews 16(3): 463-496. DOI: 10.1128/CMR.16.3.463-496.2003

187) Smith NH. 2006. A re-evaluation of M. prototuberculosis. PLoS Pathogens 2(9): e98. DOI: 10.1371/journal.ppat.0020098

188) Smith AB, Smirniotopoulos JG, Horkanyne-Szakaly I. 2013. From the radiologic pathology archives: Intraventricular Neoplasms: Radiologic-pathologic correlation. RadioGraphics 33(1): 21-43. DOI: 10.1148/rg.331125192

189) Sorrel É, Sorrel-Dejerine Y. 1932. Tuberculose osseuse et ostéo-articulaire. Volume 3. Masson: Paris, France; $381-470$.

190) Sparacello VS, Roberts CA, Kerudin A, Müller R. 2017. A 6500-year-old Middle Neolithic child from Pollera Cave (Liguria, Italy) with probable multifocal osteoarticular tuberculosis. International Journal of Paleopathology 17: 67-74. DOI: 10.1016/j.ijpp.2017.01.004

191) Spekker O, Pálfi Gy, Kozocsay G, Pósa A, Bereczki Zs, Molnár E. 2012. New cases of probable skeletal tuberculosis from the Neolithic period of Hungary - A morphological study. Acta Biologica Szegediensis 56(2): 115-123.

192) Spekker O, Pálfi Gy, Bereczki Zs, Molnár E. 2014. Paleopathological analysis of skeletal remains from a $10^{\text {th }}-12^{\text {th }}$ century AD cemetery from Hungary. In 19th Congress of the European Anthropological Association. Anthropology: Unity in Diversity (Moscow, Russia, August 25-29, 2014), Abstracts, Godina E, Kharitonov V (eds.). Moscow University Press: Moscow, Russia; 110.

193) Spekker O, Mészáros K, Paja L, Bereczki Zs, Molnár E, Pálfi Gy. 2016. Examination of tuberculosis related pathological lesions in the Late Bronze Age skeletal series of Tápé-Széntéglaégető - Preliminary 
results. In IUAES Inter-Congress. World anthropologies and privatization of knowledge: engaging anthropology in public, (Dubrovnik, Croatia, May 4-9, 2016), Abstract book. Dubrovnik, Croatia; 336.

194) Spekker O, Hunt DR, Váradi OA, Berthon W, Molnár E, Pálfi Gy. 2018. Rare manifestations of spinal tuberculosis in the Robert J. Terry Anatomical Skeletal Collection (National Museum of Natural History, Smithsonian Institution, Washington, DC, USA). International Journal of Osteoarchaeology, accepted.

195) Spiegel DA, Singh GK, Banskota AK. 2005. Tuberculosis of the musculoskeletal system. Techniques in Orthopaedics 20(2): 167-178.

196) Spigelman M, Lemma E. 1993. The use of the polymerase chain reaction (PCR) to detect Mycobacterium tuberculosis in ancient skeletons. International Journal of Osteoarchaeology 3(2):137-143. DOI: 10.1002/oa.1390030211

197) Sridhar K, Krishnan P. 2009. Complete posterior migration of intact vertebral body in spinal tuberculosis. Neurology India 57(4): 483-485. DOI: 10.4103/0028-3886.55600

198) Stone AC, Wilbur AK, Buikstra JE, Roberts CA. 2009. Tuberculosis and leprosy in perspective. American Journal of Physical Anthropology 140(Suppl. 49): 66-94. DOI: 10.1002/ajpa.21185

199) Strouhal E. 1999. Ancient Egypt and tuberculosis. In Tuberculosis: past and present, Pálfi Gy, Dutour O, Deák J, Hutás I (eds). TB Foundation: Szeged, Hungary \& Golden Book Publisher: Budapest, Hungary; $451-460$.

200) Suzuki T, Inoue T. 2007. Earliest evidence of spinal tuberculosis from the Aneolithic Yayoi period in Japan. International Journal of Osteoarchaeology 17(4): 392-402. DOI: 10.1002/oa.871

\section{T}

201) Taheri MS, Karimi MA, Haghighatkhah H, Pourghorban R, Samadian M, Kasmaei HD. 2015. Central nervous system tuberculosis: an imaging-focused review of a reemerging disease. Radiology Research and Practice DOI: 10.1155/2015/202806

202) Templin O, Schultz M. 1994. Evidence of tuberculosis in the medieval infant population from Bettingen (Switzerland). Homo 45(Suppl. 1): S130.

203) Teschler-Nicola M, Wiltschke-Schrotta K, Prossinger H, Berner M. 1994. The epidemiology of an early medieval population from Gars/Thunau, Lower Austria. Homo 45(Suppl. 1): 131.

204) Teschler-Nicola M, Novotny F, Spannagl-Steiner M, Stadler P, Prohaska T, Irrgeher J, Zitek A, Däubl B, Haring E, Rumpelmayr K, Wild EM. 2015. The early medieval manorial estate of Gars/Thunau, Lower Austria: an enclave of endemic tuberculosis?. Tuberculosis 95(Suppl. 1): S51-S59. DOI: 10.1016/j.tube.2015.02.017

205) Thwaites GE. 2013. Tuberculous meningitis. Medicine 41(12): 683-685. DOI: 10.1016/j.mpmed.2013.09.006

206) Thwaites GE, Tran TH. 2005. Tuberculous meningitis: many questions, too few answers. Lancet Neurology 4(3): 160-170. DOI: 10.1016/S1474-4422(05)01013-6 
207) Thwaites GE, Chau TTH, Stepniewska K, Phu NH, Chuong LV, Sinh DX, White NJ, Parry CM, Farrar JJ. 2002. Diagnosis of adult tuberculous meningitis by use of clinical and laboratory features. Lancet 360(9342): 1287-1292. DOI: 10.1016/S0140-6736(02)11318-3

208) Thwaites GE, Fisher M, Hemingway C, Scott G, Solomon T, Innes J. 2009. British Infection Society guidelines for the diagnosis and treatment of tuberculosis of the central nervous system in adults and children. Journal of Infection 59(3): 167-187. DOI: 10.1016/j.jinf.2009.06.011

209) van Toorn R, Solomons R. 2014. Update on the diagnosis and management of tuberculous meningitis in children. Seminars Pediatric Neurology 21(1): 12-18. DOI: 10.1016/j.spen.2014.01.006

210) Traub M, Leake J, Scholtz C, Thakkar C. 1986. Chronic untreated tuberculous meningitis. Journal of Neurology 233(4): 254-256. DOI: 10.1007/BF00314030

211) Tseng C-C, Huang R-M, Chen K-T. 2014. Tuberculosis arthritis: epidemiology, diagnosis, treatment. Clinical Research on Foot and Ankle 2(2): 131. DOI: 10.4172/2329-910X.1000131

212) Turliuc DM, Cucu AI, Dumitrescu GF, Dobrovăţ B, Popescu CE, Costăchescu B, Roata C, Romedea SN, Sava A, Costea CF. 2016. Giant tuberculous iliopsoas abscess secondary to Pott's spine. Romanian Journal of Functional and Clinical, Macro- and Microscopical Anatomy and of Anthropology 15(2): 222228.

213) Tyagi DK, Balasubramaniam S, Jayaswal SA, Savant HV, Gandhi AS. 2016. Outcome analysis of ventriculoperitoneal shunt procedures in hydrocephalus due to tubercular meningitis and non-infective causes. International Journal of Contemporary Pediatrics 3(4): 1210-1215. DOI: 10.18203/23493291.ijcp20162788

V

214) Vanhoenacker FM, Sanghvi DA, De Backer AI. 2009. Imaging features of extraaxial musculoskeletal tuberculosis. Indian Journal of Radiology and Imaging 19(3): 176-186. DOI: 10.4103/0971-3026.54873

215) Vasa M, Ohikhuare C, Brickner L. 2009. Primary sternal tuberculosis osteomyelitis: a case report and discussion. Canadian Journal of Infectious Diseases and Medical Microbiology 20(4): e181-e184.

216) Vinnard C, Macgregor RR. 2009. Tuberculous meningitis in HIV-infected individuals. Current HIV/AIDS Reports 6(3): 139-145.

217) Vita S, Ajassa C, Caraffa E, Lichtner M, Mascia C, Mengoni F, Paglia MG, Mancarella C, Colistra D, Di Biasi C, Ciardi RM, Mastroianni CM, Vullo V. 2017. Immunological diagnosis as an adjunctive tool for an early diagnosis of tuberculous meningitis of an immune competent child in a low tuberculosis endemic country: a case report. BMC Research Notes 10: 123. DOI: 10.1186/s13104-017-2444-9

\section{W}

218) Winland K, Rothschild BM, Rothschild C. 1997. Reproducibility of patterns of tuberculosis-related periosteal reaction in the Hamman-Todd, Grant \& Terry Collections. American Journal of Physical Anthropology Suppl. 24: 243. 
219) Wiratnaya IGE, Susila IWRB, Sindhughosa DA. 2017. Tuberculous osteomyelitis mimicking a lytic bone tumor: report of two cases and literature review. Revista Brasileira de Ortopedia (English Edition) DOI: 10.1016/j.rboe.2017.11.015

220) Wirth T, Hildebrand F, Allix-Béguec C, Wölbeling F, Kubica T, Kremer K, van Soolingen D, RüschGerdes S, Locht C, Brisse S, Meyer A, Supply P, Niemann S. 2008. Origin, spread and demography of the Mycobacterium tuberculosis complex. PLoS Pathogens 4(9): e1000160. DOI: 10.1371/journal.ppat.1000160

221) World Health Organization. 1994. TB is a low priority. In TB: a global emergency. WHO report on the TB epidemic. WHO: Geneva, Italy; 1.

222) World Health Organization. 2017. TB disease burden. In Global tuberculosis report 2017. WHO: Geneva, Italy; 21-62.

$\mathbf{Y}$

223) Yap FY, Skalski MR, Patel DB, Schein AJ, White EA, Tomasian A, Masih S, Matcuk GR. 2017. Hypertrophic osteoarthropathy: clinical and imaging features. RadioGraphics 37(1): 157-175. DOI: 10.1148/rg.2017160052

224) Yazdi T, Rahimizadeh A. 2012. An illustrative case isolated posterior spinal arch tuberculosis. World Spinal Column Journal 3(3): 98-101.

$\mathbf{Z}$

225) Zink AR, Molnár E, Motamedi N, Pálfi Gy, Marcsik A, Nerlich AG. 2007. Molecular history of tuberculosis from ancient mummies and skeletons. International Journal of Osteoarchaeology 17(4): 380-391. DOI: 10.1002/oa.909

226) Zumla A, Malon P, Henderson J, Grange JM. 2000. Impact of HIV infection on tuberculosis. Postgraduate Medical Journal 76(895): 259-268. DOI: 10.1136/pmj.76.895.259 


\section{LIST OF PUBLICATIONS}


MTMT ID: 10043233

\section{1) THE 2 PAPERS PROVIDING BASIS FOR THE DISSERTATION-}

Spekker O, Hunt DR, Váradi OA, Berthon W, Molnár E, Pálfi Gy. 2018. Rare manifestations of spinal tuberculosis in the Robert J. Terry Anatomical Skeletal Collection (National Museum of Natural History, Smithsonian Institution, Washington, DC, USA). International Journal of Osteoarchaeology, accepted.

IF 2017: 1.038

Molnár E, Donoghue HD, Lee OY-C, Wu HHT, Besra GS, Minnikin DE, Bull ID, Llewellyn G, Williams CM, Spekker O, Pálfi Gy. 2015. Morphological and biomolecular evidence for tuberculosis in $8^{\text {th }}$ century AD skeletons from Bélmegyer-Csömöki domb, Hungary. Tuberculosis 95(Suppl. 1): S35-S41. DOI: 10.1016/j.tube.2015.02.032 $\quad$ IF 2015: $\mathbf{2 . 7 1 1}^{2}$

\section{2) PEER-REVIEWED JOURNAL ARTICLES-}

Spekker O, Pálfi Gy, Kozocsay G, Pósa A, Bereczki Zs, Molnár E. 2012. New cases of probable skeletal tuberculosis from the Neolithic period of Hungary - A morphological study. Acta Biologica Szegediensis 56(2): 115-123.

Spekker O, Kasik L, Nagy L. 2013. A NAT (2012) szociális kompetencia fejlesztésére irányuló feladatainak lehetséges megvalósulása a biológiaórákon. A Biológia Tanítása 21(2): 3-14.

Spekker O, Pósa A, Pálfi Gy, Zink A, Maixner F, Bereczki Zs, Molnár E. 2013. Specifikus fertőző megbetegedés diagnózisa Vésztő Mágori-halom újkőkori leletein. Anthropologiai Közlemények 54: 77-92.

Molnár E, Donoghue HD, Lee OY-C, Wu HHT, Besra GS, Minnikin DE, Bull ID, Llewellyn G, Williams CM, Spekker O, Pálfi Gy. 2015. Morphological and biomolecular evidence for tuberculosis in $8^{\text {th }}$ century AD skeletons from Bélmegyer-Csömöki domb, Hungary. Tuberculosis 95(Suppl. 1): S35-S41. DOI: 10.1016/j.tube.2015.02.032 $\quad$ IF 2015: $\mathbf{2 . 7 1 1}^{2}$

Pósa A, Maixner F, Sola C, Bereczki Zs, Molnár E, Masson M, Lovász G, Spekker O, Wicker E, Perrin P, Dutour O, Zink A, Pálfi Gy. 2015. Tuberculosis infection in a late-medieval Hungarian population. Tuberculosis 95(Suppl. 1): S60-S64. DOI: 10.1016/j.tube.2015.02.010 IF 2015: 2.711

Spekker O, Hunt DR, Váradi OA, Berthon W, Molnár E, Pálfi Gy. 2018. Rare manifestations of spinal tuberculosis in the Robert J. Terry Anatomical Skeletal Collection (National Museum of Natural History, Smithsonian Institution, Washington, DC, USA). International Journal of Osteoarchaeology, accepted.

IF 2017: 1.038

TOTAL IF: $\quad 6.46$

\section{3) PAPERS PUBLISHED IN CONFERENCE PROCEEDINGS-}

Spekker O, Kozocsay G, Bereczki Zs, Pálfi Gy, Molnár E. 2012. Csonttuberkulózis nyomai Vésztő Mágorihalom neolit kori temetöben. In Mesterházy B (ed.): $11^{\text {th }}$ International Conference on Applications of Natural, Technological and Economic Sciences (Szombathely, Hungary, 19 May 2012). Presentations. Nyugatmagyarországi Egyetem: Szombathely, Hungary; 208-213.

Spekker O, Pálfi Gy, Molnár E. 2014. Csont-ízületi tuberkulózis nyomai Vésztő Mágori-halom neolit kori temetőben. In Vágvölgyi Cs, Szekeres A (eds.): A biológia jövője, a jövő biológusai. Avagy szemelvények a magyarországi felsőoktatási intézményekben végzett tudományos munka eredményeiröl. Válogatás a XXXI. Országos Tudományos Diákköri Konferencia Biológia szekciójának dolgozataiból. JATEPress: Szeged, Hungary; 69-80. 
Pálfi Gy, Spekker O, Marcsik A, Paja L, Balázs J, Maixner F, Zink A, Palkó A, Dutour O, Donoghue HD, Lee OY-C, Wu HHT, Besra GS, Minnikin DE, Bull ID, Llewellyn G, Williams CM, Nerlich A, Molnár E. 2016. Tuberculosis paleopathology research in the Szeged Anthropological Collection: new data from the Avar Age. In Gál SzS (ed.): The talking dead. New results from Central and Eastern European osteoarchaeology. Proceedings of the First International Conference of the Török Aurél Anthropological Association from Târgu Mureş. Mega Publishing House: Cluj-Napoca, Romania; 79-109.

Váradi OA, Kecskeméti A, Spekker O, Molnár E, Bereczki Zs, Szekeres A, Vágvölgyi Cs, Pálfi Gy. 2016. Cases of tuberculosis infection verified by lipid biomarker analysis in Hungarian archaeological samples. In Gál SzS (ed.): The talking dead. New results from Central and Eastern European osteoarchaeology. Proceedings of the First International Conference of the Török Aurél Anthropological Association from Târgu Mureş. Mega Publishing House: Cluj-Napoca, Romania; 129-142.

\section{4) CONFERENCE PRESENTATIONS-}

\section{A) National conferences-}

Spekker O, Molnár E, Marcsik A, Pálfi Gy. 2011. Daganatos megbetegedések előfordulása magyarországi történeti népességek körében. In „Hadak útján”. A Népvándorláskor Fiatal Kutatóinak XXI. Konferenciája (Szeged, Hungary, 23-25 November 2011). Abstract Book. Szeged, Hungary; 6-7.

Spekker O. 2012. Csont-ízületi tuberkulózis nyomai Vésztő Mágori-halom neolit kori temetöben. In Scientific Students' Association Conference (Szeged, Hungary, 15 November 2012). - Special Award

Spekker O. 2013. Csont-ízületi tuberkulózis nyomai Vésztő Mágori-halom neolit kori temetőben. In $31^{s t}$ National Scientific Students' Association Conference (Szeged, Hungary, 2-4 April 2013). - $\mathbf{2}^{\text {nd }}$ place; JATEPress Publication Award

Spekker O, Pálfi Gy, Bereczki Zs, Paja L, Molnár E. 2013. Hendikeppel az avar korban - A mozgáskorlátozottság hatása a csontokra. In „Hadak útján”. A Népvándorláskor Fiatal Kutatóinak XXIII. Konferenciája (Veszprém, Hungary, 2-4 October 2013). Abstract Book. Laczkó Dezső Múzeum: Veszprém, Hungary; 11.

Spekker O, Paja L, Hunt DR, Ortner DJ, Dutour O, Molnár E, Pálfi Gy. 2017. A tuberkulózissal összefüggésbe hozható endocranialis elváltozások makromorfológiai jellegzetességeinek, előfordulásának és együttes megjelenésének vizsgálata a washingtoni Terry Collection-ben. In A Magyar Biológiai Társaság Embertani Szakosztályának 389. ülése Dr. Farkas L. Gyula professzor emeritus, az MBT Embertani Szakosztályának elnöke 85. születésnapja tiszteletére (Szeged, Hungary, 5 May 2017).

\section{B) International conferences-}

Spekker O, Kozocsay G, Pálfi Gy, Molnár E. 2012. Probable cases of skeletal tuberculosis from the Neolithic period of Hungary - A morphological study. In Pálfi Gy, Bereczki Zs, Molnár E, Dutour O (eds.): ICEPT-2. The Past and Present of Tuberculosis: A Multidisciplinary Overview on the Origin and Evolution of TB (Szeged, Hungary, 22-25 March 2012). Program and Abstracts of the 2012 TB Evolution Meeting. Szeged University Press: Szeged, Hungary; 91.

Spekker O, Kozocsay G, Bereczki Zs, Pálfi Gy, Molnár E. 2012. Csonttuberkulózis nyomai Vésztő Mágorihalom neolit kori temetöben. In Mesterházy B (ed.): $11^{\text {th }}$ International Conference on Applications of Natural, Technological and Economic Sciences (Szombathely, Hungary, 19 May 2012). Presentations. Nyugatmagyarországi Egyetem: Szombathely, Hungary; 5.

Spekker O, Molnár E, Pósa A, Maixner F, Zink A, Pálfi Gy. 2013. Paleopathological study of some Neolithic cases from Hungary. In Coqueugniot H, Dutour O (eds.): Groupe des Paleopathologistes de Langue Française. Colloque 2013 (Toulon, France, 12-13 April 2013). Volume des Resumes. Centre archéologique du Var: Toulon, France; 16. 
Spekker O, Molnár E, Bereczki Zs, Pósa A, Maixner F, Zink A, Pálfi Gy. 2013. Paleopathological study of Neolithic TB cases from Hungary. In German Society of Anthropology (GfA) $10^{\text {th }}$ International Meeting. Biological Anthropology: Prospects and Perspectives (Bolzano, Italy, 2-6 September 2013), Program and Abstracts. Institute for Mummies and the Iceman, EURAC Research: Bolzano, Italy; 98.

Pálfi Gy, Marcsik A, Pap I, Kustár Á, Donoghue HD, Minnikin DE, Lee OY-C, Wu HHT, Besra GS, Bereczki Zs, Spekker O, Molnár E. 2014. Osteoarchaeological and paleomicrobiological evidence of leprosy in Hungary. (The 83 ${ }^{\text {rd }}$ Annual Meeting of the American Association of Physical Anthropologists (AAPA) (Calgary, Canada, 8-12 April 2014)). American Journal of Physical Anthropology 153: 213.

Spekker O, Pálfi Gy, Bereczki Zs, Molnár E. 2014. Paleopathological analysis of skeletal remains from a $10^{\text {th }}$ $12^{\text {th }}$ century AD cemetery from Hungary. In Godina E, Kharitonov V (eds.): $19^{\text {th }}$ Congress of the European Anthropological Association. Anthropology: Unity in Diversity (Moscow, Russia, 25-29 August 2014). Abstracts. Moscow University Press: Moscow, Russia; 110.

Spekker O, Masson M, Molnár E, Marcsik A, Pósa A, Lovász G, Mészáros K, Neparáczki E, Maczel M, Bereczki Zs, Paja L, Pálfi Gy. 2015. Nouveaux cas de tuberculose squelettique dans la collection anthropologique de Szeged: résultats du programme de recherche 2008-2014. In Groupe des Paleopathologistes de Langue Française. Colloque 2015 (Bordeaux, France, 13-14 March 2015). Programme. Université de Bordeaux: Bordeaux, France; 20.

Spekker O, Lovász G, Masson M, Pósa A, Mészáros K, Váradi OA, Neparáczki E, Maczel M, Marcsik A, Schultz M, Nerlich A, Minnikin DE, Donoghue HD, Maixner F, Zink A, Dutour O, Paja L, Bereczki Zs, Molnár E, Pálfi Gy. 2015. On the traces of ancient tuberculosis: a preliminary summary of the tuberculosis paleopathology research in the Szeged Anthropological Collection from the 1970's to the present day. In $11^{\text {th }}$ Meeting of the Society for Anthropology (GfA). Evolutionary and Modern Challenges to Homo sapiens - An Anthropological Inquiry (Munich, Germany, 15-18 September 2015). Abstract and Program Booklet. Munich, Germany; 100-101. - Poster Presentation Award

Pálfi Gy, Molnár E, Bereczki Zs, Spekker O, Pósa A, Zink A, Dutour O, Marcsik A. 2015. Summary of the tuberculosis paleopathology research in the Szeged Anthropological Collection from the 1970's to the present day. In $1^{\text {st }}$ Conference of the Anthropological Association „Aurél Török” (Târgu Mureș, Romania, 13-15 November 2015). Târgu Mureș, Romania; 8-9.

Spekker O, Molnár E, Bereczki Zs, Pósa A, Maixner F, Zink A, Pálfi Gy. 2015. New cases of probable skeletal tuberculosis from the Neolithic tell settlement of Vésztő-Mágor, Hungary. In $1^{\text {st }}$ Conference of the Anthropological Association „Aurél Török” (Târgu Mureș, Romania, 13-15 November 2015). Târgu Mureș, Romania; 9.

Spekker O, Molnár E, Lovász G, Marcsik A, Masson M, Bereczki Zs, Paja L, Balázs J, Váradi OA, Neparáczki E, Pósa A, Maixner F, Zink A, Perrin P, Coqueugniot H, Dutour O, Pálfi Gy. 2016. Paléopathologie infectieuse chez des sujets immatures: exemple de la tuberculose. Résultats de 45 ans de recherche effectuée dans la collection anthropologique de Szeged. In Groupe des Paleopathologistes de Langue Française. Colloque 2016 (Toulouse, France, 11-12 March 2016). Programme and Volume des Résumés. Faculté de médicine Purpan, Université de Paul Sabatier Toulouse: Toulouse, France; 19.

Pálfi Gy, Molnár E, Marcsik A, Pósa A, Spekker O, Pap I, Szikossy I, Hajdu T, Minnikin DE, Lee OY-C, Donoghue HD, Spigelman M, Dutour O, Coqueugniot H, Maixner F, Zink A. 2016. Paleopathology of tuberculosis and other mycobacterial infections in Hungary. In IUAES Inter-Congress. World Anthropologies and Privatization of Knowledge: Engaging Anthropology in Public (Dubrovnik, Croatia, 4-9 May 2016). Abstract Book. Dubrovnik, Croatia; 127.

Spekker O, Mészáros K, Paja L, Bereczki Zs, Molnár E, Pálfi Gy. 2016. Examination of tuberculosis related pathological lesions in the Late Bronze Age skeletal series of Tápé-Széntéglaégető - Preliminary results. In IUAES Inter-Congress. World Anthropologies and Privatization of Knowledge: Engaging Anthropology in Public (Dubrovnik, Croatia, 4-9 May 2016). Abstract Book. Dubrovnik, Croatia; 336. 
Váradi OA, Kecskeméti A, Spekker O, Molnár E, Bereczki Zs, Szekeres A, Vágvölgyi Cs, Pálfi Gy. 2016. Mycobacterium tuberculosis komplex kimutatása lipid biomarkerek segítségével. In Füzesi I, Kovács E, Puskás J (ed.): $15^{\text {th }}$ International Conference on Applications of Natural, Technological and Economic Sciences (Szombathely, Hungary, 14 May 2016). Abstracts of the presentations. Nyugat-magyarországi Egyetem: Szombathely, Hungary; 28.

Spekker O, Molnár E, Marcsik A, Lovász G, Masson M, Maczel M, Pósa A, Neparáczki E, Váradi OA, Schultz M, Nerlich A, Minnikin DE, Donoghue HD, Maixner F, Zink A, Dutour O, Bereczki Zs, Paja L, Pálfi Gy. 2016. On the traces of ancient tuberculosis: possibilities of the macromorphological diagnosis of tuberculosis in prehistoric and historic osteological series - Skeletal tuberculosis cases from the Szeged Anthropological Collection. In Working Your Fingers to the Bone - An Interdisciplinary Conference on Identifying Occupation from the Skeleton (Coimbra, Portugal, 6-8 July 2016). Program and Abstract Book. Department of Life Sciences, University of Coimbra: Coimbra, Portugal; 46.

Tihanyi B, Révész L, Berthon W, Dutour O, Nepper I, Spekker O, Bereczki Zs, Molnár E, Pálfi Gy. 2016. The Hungarian Conquest period archery and activity-induced stress markers - Anthropological and archaeometrical studies of a $10^{\text {th }} \mathrm{c}$. AD Hungarian series. In Working Your Fingers to the Bone - An Interdisciplinary Conference on Identifying Occupation from the Skeleton (Coimbra, Portugal, 6-8 July 2016). Program and Abstract Book. Department of Life Sciences, University of Coimbra: Coimbra, Portugal; 50.

Pálfi Gy, Dutour O, Ortner DJ, Hunt DR, Paja L, Molnár E, Spekker O. 2017. Distribution et association des différents types d'altérations endocrâniennes liées à la tuberculose dans «The Robert J. Terry Anatomical Skeletal Collection» (National Museum of Natural History, Smithsonian Institution, Washington, DC, USA). In Groupe des Paleopathologistes de Langue Française. Colloque 2017 (Caen, France, 24-25 March 2017). Programme. Université de Caen Normandie: Caen, France; 7.

Spekker O, Dutour O, Ortner DJ, Hunt DR, Paja L, Molnár E, Pálfi Gy. 2017. Aspect et co-occurrence de différents types d'attérations endocrâniennes chez des individus décédés de la tuberculose Exemptes issus de la «Robert J. Terry Anatomical Sketetal Collection» (Washington, DC, USA). In Groupe des Paleopathologistes de Langue Française. Colloque 2017 (Caen, France, 24-25 March 2017). Programme. Université de Caen Normandie: Caen, France; 28.

Molnár E, Marcsik A, Pap I, Bereczki Zs, Spekker O, Balázs J, Szeniczey T, Köhler K, Hajdu T, Pálfi Gy. 2017. Skeletal evidence of leprosy from ancient times in the territory of present-day Hungary. In $2^{\text {nd }}$ Conference of the 'Török Aurél' Anthropological Association - Past and Present of Biological Anthropology - The Heritage of Török Aurél's Oeuvre (Târgu Mureș, Romania, 13-15 October 2017). Program and Abstracts. Târgu Mureș, Romania; 19-20.

Spekker O, Paja L, Hunt DR, Ortner DJ, Dutour O, Molnár E, Pálfi Gy. 2017. Examples of different types of endocranial bony changes probably related to tuberculosis from the Robert J. Terry Anatomical Skeletal Collection (Washington, DC, USA). In $2^{\text {nd }}$ Conference of the 'Török Aurél' Anthropological Association Past and Present of Biological Anthropology - The Heritage of Török Aurél's Oeuvre (Târgu Mureș, Romania, 13-15 October 2017). Program and Abstracts. Târgu Mureș, Romania; 23-24. 


\begin{abstract}
APPENDIX
Supplementary table 1: Basic biographic data of specimens in the TB group. 170

Supplementary table 2: Basic biographic data of specimens in the NTB group 192

Supplementary table 3: Individual data regarding TB-related endocranial alterations in the TB group......214

Supplementary table 4: Individual data regarding TB-related endocranial alterations in the NTB group...220

Supplementary table 5: Individual data regarding TB-related non-endocranial bony changes in the TB group .......225

Supplementary table 6: Individual data regarding TB-related non-endocranial bony changes in the NTB group ....239
\end{abstract}


Supplementary table 1: Basic biographic data of specimens in the TB group $(\mathrm{N}=302)$.


$+=$ included into the evaluation considering TBM-related endocranial alterations; $-=$ excluded from the evaluation considering TBM-related endocranial lesions).

\begin{tabular}{|c|c|c|c|c|c|c|c|c|}
\hline \multirow{2}{*}{ No. } & \multirow{2}{*}{ Terry No. } & \multirow{2}{*}{$\begin{array}{c}\text { Age } \\
\text { at death }\end{array}$} & \multirow{2}{*}{ Sex } & \multicolumn{4}{|c|}{ Cause of death } & \multirow{2}{*}{$\begin{array}{l}\text { Included or not } \\
\text { into the evaluation }\end{array}$} \\
\hline & & & & MR & DC1 & DC2 & DC3 & \\
\hline 1 & 12 & c. 61 years & $\mathbf{F}$ & TB & Pulmonary TB & - & - & - \\
\hline 2 & 13R & 28 years & $\mathbf{M}$ & Pulmonary TB & - & $\begin{array}{c}\text { Infection } \\
\text { of the right toe }\end{array}$ & - & + \\
\hline 3 & 17R & c. 66 years & $\mathbf{F}$ & Pulmonary TB & Pneumonia & Pneumonitis & $\begin{array}{c}\text { Psychosis, } \\
\text { arteriosclerosis }\end{array}$ & - \\
\hline 4 & $23 R$ & 49 years & $\mathbf{F}$ & Pulmonary TB & - & - & - & + \\
\hline 5 & $30 R$ & 26 years & $\mathbf{M}$ & TB & - & TB meningitis & - & + \\
\hline 6 & $35 R$ & 60 years & $\mathbf{M}$ & Pulmonary TB & - & - & - & + \\
\hline 7 & 39 & 22 years & $\mathbf{F}$ & $\begin{array}{c}\text { TB, } \\
\text { pneumonia }\end{array}$ & Pneumonia & - & - & + \\
\hline 8 & 54 & 36 years & $\mathbf{M}$ & $\begin{array}{c}\text { TB, } \\
\text { diarrhoea }\end{array}$ & Pulmonary TB & - & - & + \\
\hline 9 & 84 & c. 24 years & $\mathbf{M}$ & Pulmonary TB & Pulmonary TB & - & - & + \\
\hline 10 & $87 R$ & 73 years & $\mathbf{F}$ & Lues & Lues & Suspected TB & - & + \\
\hline 11 & $89 R$ & c. 32 years & $\mathbf{M}$ & TB & - & - & - & + \\
\hline 12 & 90 & 18 years & M & - & Ichthyosis & Pulmonary TB & - & + \\
\hline
\end{tabular}




\begin{tabular}{|c|c|c|c|c|c|c|c|c|}
\hline \multirow{2}{*}{ No. } & \multirow{2}{*}{ Terry No. } & \multirow{2}{*}{$\begin{array}{c}\text { Age } \\
\text { at death }\end{array}$} & \multirow{2}{*}{ Sex } & \multicolumn{4}{|c|}{ Cause of death } & \multirow{2}{*}{$\begin{array}{l}\text { Included or not } \\
\text { into the evaluation }\end{array}$} \\
\hline & & & & MR & DC1 & DC2 & DC3 & \\
\hline 13 & $91 R$ & c. 65 years & $\mathbf{F}$ & Pulmonary condition & Pulmonary TB & - & - & + \\
\hline 14 & 95 & c. 35 years & $\mathbf{F}$ & Pulmonary TB & - & - & - & + \\
\hline 15 & $95 R$ & c. 30 years & $\mathbf{M}$ & Pulmonary TB & - & - & - & + \\
\hline 16 & $103 R$ & c. 74 years & $\mathbf{F}$ & - & Emphysema & TB & - & + \\
\hline 17 & 114 & 43 years & $\mathbf{F}$ & TB & - & - & - & + \\
\hline 18 & 125 & 31 years & M & Pulmonary TB & - & - & - & - \\
\hline 19 & 128 & 21 years & $\mathbf{M}$ & TB & Pulmonary TB & - & - & + \\
\hline 20 & 129 & 18 years & $\mathbf{M}$ & TB spondylitis & - & - & - & + \\
\hline 21 & 130 & 58 years & $\mathbf{M}$ & Pellagra & Pulmonary TB & - & Inanition & + \\
\hline 22 & 138 & 49 years & $\mathbf{M}$ & Pulmonary phthisis & Pulmonary TB & - & - & + \\
\hline 23 & 139 & 33 years & $\mathbf{F}$ & TB & Pulmonary TB & - & - & + \\
\hline 24 & 145R & 31 years & $\mathbf{M}$ & Pulmonary TB & - & - & - & + \\
\hline 25 & 146R & 30 years & $\mathbf{F}$ & Pulmonary TB & Pulmonary TB & - & Nephritis & + \\
\hline 26 & $158 R$ & c. 32 years & $\mathbf{F}$ & TB & Pulmonary TB & - & - & + \\
\hline
\end{tabular}




\begin{tabular}{|c|c|c|c|c|c|c|c|c|}
\hline \multirow{2}{*}{ No. } & \multirow{2}{*}{ Terry No. } & \multirow{2}{*}{$\begin{array}{c}\text { Age } \\
\text { at death }\end{array}$} & \multirow{2}{*}{ Sex } & \multicolumn{4}{|c|}{ Cause of death } & \multirow{2}{*}{$\begin{array}{l}\text { Included or not } \\
\text { into the evaluation }\end{array}$} \\
\hline & & & & MR & DC1 & DC2 & DC3 & \\
\hline 27 & 182 & 43 years & $\mathbf{M}$ & Pulmonary TB & Pulmonary TB & - & - & + \\
\hline 28 & 194 & 73 years & M & Pulmonary TB & - & - & - & + \\
\hline 29 & 199R & c. 58 years & $\mathbf{F}$ & Pulmonary TB & - & - & - & - \\
\hline 30 & 204 & 24 years & $\mathbf{M}$ & Pulmonary TB & - & - & - & + \\
\hline 31 & 205 & 42 years & $\mathbf{M}$ & Pulmonary TB & Pulmonary TB & - & - & + \\
\hline 32 & 207 & 45 years & $\mathbf{M}$ & Pulmonary TB & - & - & - & + \\
\hline 33 & 220 & c. 56 years & $\mathbf{M}$ & Pulmonary TB & - & - & - & + \\
\hline 34 & 222 & 20 years & M & Pulmonary TB & Pulmonary TB & - & - & + \\
\hline 35 & 230 & 37 years & M & Pulmonary TB & - & - & - & + \\
\hline 36 & 232R & c. 35 years & $\mathbf{F}$ & Pulmonary TB & - & - & - & + \\
\hline 37 & 235 & 29 years & $\mathbf{M}$ & - & Miliary TB & - & - & + \\
\hline 38 & 248R & 70 years & $\mathbf{F}$ & Possible TB & Pneumonia & - & - & + \\
\hline 39 & 250 & 52 years & $\mathbf{M}$ & $\begin{array}{c}\text { Laparotomy, } \\
\text { stomach carcinoma }\end{array}$ & TB peritonitis & - & - & + \\
\hline 40 & 251 & 34 years & M & TB & Pulmonary TB & - & - & + \\
\hline
\end{tabular}




\begin{tabular}{|c|c|c|c|c|c|c|c|c|}
\hline \multirow{2}{*}{ No. } & \multirow{2}{*}{ Terry No. } & \multirow{2}{*}{$\begin{array}{c}\text { Age } \\
\text { at death }\end{array}$} & \multirow{2}{*}{ Sex } & \multicolumn{4}{|c|}{ Cause of death } & \multirow{2}{*}{$\begin{array}{l}\text { Included or not } \\
\text { into the evaluation }\end{array}$} \\
\hline & & & & MR & DC1 & DC2 & DC3 & \\
\hline 41 & 254 & 21 years & $\mathbf{M}$ & Pulmonary TB & - & - & - & + \\
\hline 42 & 255 & 22 years & $\mathbf{F}$ & Pulmonary TB & - & - & - & + \\
\hline 43 & 264 & c. 67 years & $\mathbf{M}$ & $\begin{array}{c}\text { TB, } \\
\text { pneumonia }\end{array}$ & - & - & - & + \\
\hline 44 & 265 & 32 years & $\mathbf{M}$ & TB & - & - & - & + \\
\hline 45 & 267 & c. 42 years & $\mathbf{M}$ & Pulmonary TB & - & - & - & + \\
\hline 46 & 269 & 20 years & $\mathbf{M}$ & TB & - & - & - & + \\
\hline 47 & 270 & 55 years & $\mathbf{M}$ & TB & - & - & - & + \\
\hline 48 & 279 & 34 years & $\mathbf{M}$ & Pulmonary TB & - & - & - & + \\
\hline 49 & 280 & 24 years & $\mathbf{F}$ & $\mathbf{T B}$ & - & - & - & + \\
\hline 50 & 282 & 57 years & $\mathbf{M}$ & Myocarditis & Pulmonary TB & Myocarditis & - & + \\
\hline 51 & 283R & 40 years & $\mathbf{M}$ & $\begin{array}{c}\text { TB, } \\
\text { haemorrhage }\end{array}$ & Pulmonary TB & - & - & + \\
\hline 52 & 284 & c. 67 years & $\mathbf{M}$ & TB & - & - & - & + \\
\hline 53 & 304 & 20 years & $\mathbf{F}$ & - & Pulmonary TB & - & - & + \\
\hline 54 & 306 & 18 years & $\mathbf{F}$ & Pulmonary TB & - & - & - & + \\
\hline
\end{tabular}

- 173 - 


\begin{tabular}{|c|c|c|c|c|c|c|c|c|}
\hline \multirow{2}{*}{ No. } & \multirow{2}{*}{ Terry No. } & \multirow{2}{*}{$\begin{array}{c}\text { Age } \\
\text { at death }\end{array}$} & \multirow{2}{*}{ Sex } & \multicolumn{4}{|c|}{ Cause of death } & \multirow{2}{*}{$\begin{array}{l}\text { Included or not } \\
\text { into the evaluation }\end{array}$} \\
\hline & & & & MR & DC1 & DC2 & DC3 & \\
\hline 55 & 309 & 45 years & $\mathbf{M}$ & Pulmonary TB & Pulmonary TB & TB spondylitis & $\begin{array}{l}\text { Mastoiditis, } \\
\text { sinusitis }\end{array}$ & + \\
\hline 56 & 311R & 27 years & $\mathbf{M}$ & TB & Epilepsy & Pulmonary TB & - & - \\
\hline 57 & 318 & 45 years & $\mathbf{M}$ & $\begin{array}{c}\text { Pulmonary TB, } \\
\text { myocarditis }\end{array}$ & Pulmonary TB & - & - & + \\
\hline 58 & 328R & 65 years & $\mathbf{F}$ & $\begin{array}{l}\text { Possible TB, } \\
\text { pleurisy }\end{array}$ & Pulmonary carcinoma & Undetermined & - & + \\
\hline 59 & 329 & 18 years & $\mathbf{M}$ & $\begin{array}{c}\text { Pulmonary TB, } \\
\text { syphilis }\end{array}$ & Pulmonary TB & Syphilis & - & + \\
\hline 60 & 341 & 38 years & $\mathbf{F}$ & Pulmonary TB & - & - & - & + \\
\hline 61 & 353 & c. 37 years & $\mathbf{M}$ & - & TB peritonitis & - & - & + \\
\hline 62 & $358 R$ & c. 36 years & $\mathbf{M}$ & Pulmonary TB & - & - & - & + \\
\hline 63 & 382R & 26 years & $\mathbf{M}$ & Pulmonary TB & - & - & - & + \\
\hline 64 & 385 & 21 years & $\mathbf{M}$ & - & Pulmonary TB & - & - & + \\
\hline 65 & 386R & 66 years & $\mathbf{M}$ & Pulmonary TB & - & - & - & + \\
\hline 66 & 395 & 57 years & $\mathbf{M}$ & TB ulcers of the leg & Myocarditis & - & - & - \\
\hline 67 & 400 & c. 44 years & $\mathbf{M}$ & TB & - & - & - & + \\
\hline 68 & 402 & c. 50 years & $\mathbf{M}$ & TB & - & - & - & + \\
\hline
\end{tabular}

- 174 - 


\begin{tabular}{|c|c|c|c|c|c|c|c|c|}
\hline \multirow{2}{*}{ No. } & \multirow{2}{*}{ Terry No. } & \multirow{2}{*}{$\begin{array}{c}\text { Age } \\
\text { at death }\end{array}$} & \multirow{2}{*}{ Sex } & \multicolumn{4}{|c|}{ Cause of death } & \multirow{2}{*}{$\begin{array}{l}\text { Included or not } \\
\text { into the evaluation }\end{array}$} \\
\hline & & & & MR & DC1 & DC2 & DC3 & \\
\hline 69 & $410 R$ & 35 years & M & - & Pulmonary TB & - & - & + \\
\hline 70 & 413 & 55 years & $\mathbf{M}$ & Pulmonary TB & - & - & - & - \\
\hline 71 & 414 & 39 years & $\mathbf{M}$ & Pulmonary TB & - & - & - & - \\
\hline 72 & 423 & c. 24 years & $\mathbf{M}$ & $\begin{array}{c}\text { TB, } \\
\text { pericarditis }\end{array}$ & - & - & - & + \\
\hline 73 & 424 & c. 30 years & $\mathbf{M}$ & $\begin{array}{c}\mathrm{TB}, \\
\text { silicosis }\end{array}$ & - & - & - & + \\
\hline 74 & $426 R$ & 70 years & M & Cardiorenal disease & Arteriosclerosis & Pulmonary TB & Diabetes mellitus & + \\
\hline 75 & 432 & 67 years & $\mathbf{M}$ & $\begin{array}{l}\text { Destructive } \\
\text { nasal lesion }\end{array}$ & TB abscess & Pulmonary TB & - & + \\
\hline 76 & 434 & 41 years & $\mathbf{M}$ & Pulmonary TB & - & - & - & - \\
\hline 77 & 444 & c. 26 years & $\mathbf{M}$ & TB & Pulmonary TB & - & - & + \\
\hline 78 & 466 & 31 years & $\mathbf{M}$ & Pulmonary TB & - & - & - & + \\
\hline 79 & 468 & 23 years & $\mathbf{M}$ & $\begin{array}{l}\text { Pulmonary TB, } \\
\text { TB spondylitis }\end{array}$ & - & - & - & + \\
\hline 80 & 490 & c. 38 years & M & TB & - & Hepatic cirrhosis & - & + \\
\hline 81 & $491 R$ & $63 / 71$ years & $\mathbf{F}$ & Pulmonary TB & Arteriosclerosis & - & - & - \\
\hline 82 & 504 & c. 41 years & $\mathbf{M}$ & $\begin{array}{c}\text { TB, } \\
\text { bronchitis }\end{array}$ & Pulmonary TB & - & - & + \\
\hline
\end{tabular}

- 175 - 


\begin{tabular}{|c|c|c|c|c|c|c|c|c|}
\hline \multirow{2}{*}{ No. } & \multirow{2}{*}{ Terry No. } & \multirow{2}{*}{$\begin{array}{c}\text { Age } \\
\text { at death }\end{array}$} & \multirow{2}{*}{ Sex } & \multicolumn{4}{|c|}{ Cause of death } & \multirow{2}{*}{$\begin{array}{l}\text { Included or not } \\
\text { into the evaluation }\end{array}$} \\
\hline & & & & MR & DC1 & DC2 & DC3 & \\
\hline 83 & 508 & c. 60 years & $\mathbf{M}$ & Pulmonary TB & - & - & - & - \\
\hline 84 & 522 & 30 years & $\mathbf{M}$ & Pulmonary TB & - & - & - & + \\
\hline 85 & 523 & 47 years & $\mathbf{M}$ & Pulmonary TB & - & - & - & + \\
\hline 86 & 537 & c. 48 years & M & Possible malaria & TB meningitis & - & - & + \\
\hline 87 & 541 & 28 years & $\mathbf{F}$ & Pulmonary TB & - & - & - & + \\
\hline 88 & 549 & 33 years & $\mathbf{M}$ & Myocarditis & Pulmonary TB & - & - & + \\
\hline 89 & 555 & 56 years & $\mathbf{M}$ & Pulmonary TB & - & - & - & + \\
\hline 90 & 562 & 17 years & $\mathbf{F}$ & Pulmonary TB & - & - & - & + \\
\hline 91 & 565 & c. 32 years & $\mathbf{M}$ & Pulmonary TB & - & - & - & + \\
\hline 92 & 566 & 40 years & M & Empyema & Empyema & Possible TB & - & + \\
\hline 93 & 568 & 28 years & $\mathbf{F}$ & Pulmonary TB & - & - & - & + \\
\hline 94 & 571 & 31 years & $\mathbf{M}$ & TB & - & - & - & + \\
\hline 95 & 572 & 60 years & $\mathbf{M}$ & Pulmonary TB & - & - & - & + \\
\hline 96 & 575 & c. 47 years & $\mathbf{M}$ & Pulmonary TB & - & - & - & + \\
\hline
\end{tabular}

- 176 - 


\begin{tabular}{|c|c|c|c|c|c|c|c|c|}
\hline \multirow{2}{*}{ No. } & \multirow{2}{*}{ Terry No. } & \multirow{2}{*}{$\begin{array}{c}\text { Age } \\
\text { at death }\end{array}$} & \multirow{2}{*}{ Sex } & \multicolumn{4}{|c|}{ Cause of death } & \multirow{2}{*}{$\begin{array}{l}\text { Included or not } \\
\text { into the evaluation }\end{array}$} \\
\hline & & & & MR & DC1 & DC2 & DC3 & \\
\hline 97 & 583 & 24 years & $\mathbf{F}$ & Pulmonary TB & - & - & - & + \\
\hline 98 & 585 & 64 years & $\mathbf{M}$ & Pulmonary TB & - & - & - & + \\
\hline 99 & 592 & 25 years & $\mathbf{M}$ & Pulmonary TB & - & - & - & + \\
\hline 100 & 593 & 44 or 74 years & $\mathbf{M}$ & $\begin{array}{c}\text { TB, } \\
\text { pneumonia }\end{array}$ & Pneumonia & - & - & - \\
\hline 101 & 595 & 25 years & $\mathbf{M}$ & Pulmonary TB & - & - & - & + \\
\hline 102 & 614 & 50 years & $\mathbf{M}$ & TB & Pulmonary TB & Pneumonia & - & - \\
\hline 103 & 620 & c. 55 years & $\mathbf{M}$ & Pulmonary TB & - & Myocarditis & - & + \\
\hline 104 & $621 R$ & 29 years & $\mathbf{M}$ & Pulmonary TB & Pulmonary TB & TB emphysema & - & + \\
\hline 105 & $626 R$ & c. 56 years & $\mathbf{F}$ & Pulmonary TB & - & - & - & + \\
\hline 106 & 632 & 27 years & $\mathbf{F}$ & Pulmonary TB & - & - & - & - \\
\hline 107 & 645 & 29 years & $\mathbf{M}$ & Pulmonary TB & - & - & - & - \\
\hline 108 & 656 & 43 years & $\mathbf{M}$ & Bronchitis & Miliary TB & - & - & - \\
\hline 109 & 660 & c. 28 years & $\mathbf{M}$ & Pulmonary TB & - & - & - & - \\
\hline 110 & 663 & c. 44 years & $\mathbf{M}$ & Syphilis & Aortic aneurysm & Miliary TB & Syphilis & - \\
\hline
\end{tabular}

- 177 - 


\begin{tabular}{|c|c|c|c|c|c|c|c|c|}
\hline \multirow{2}{*}{ No. } & \multirow{2}{*}{ Terry No. } & \multirow{2}{*}{$\begin{array}{c}\text { Age } \\
\text { at death }\end{array}$} & \multirow{2}{*}{ Sex } & \multicolumn{4}{|c|}{ Cause of death } & \multirow{2}{*}{$\begin{array}{l}\text { Included or not } \\
\text { into the evaluation }\end{array}$} \\
\hline & & & & MR & DC1 & DC2 & DC3 & \\
\hline 111 & 664 & c. 48 years & $\mathbf{M}$ & Pulmonary TB & Pulmonary TB & Pneumonia & - & + \\
\hline 112 & 669R & 72 years & M & Heart disease & Pulmonary TB & Arteriosclerosis & Heart disease & + \\
\hline 113 & 675 & 27 years & M & Pulmonary TB & - & - & - & - \\
\hline 114 & 679 & 33 years & $\mathbf{F}$ & TB & - & - & - & + \\
\hline 115 & 680 & 30 years & $\mathbf{F}$ & Pulmonary TB & Pulmonary TB & TB peritonitis & - & + \\
\hline 116 & 706 & 38 years & $\mathbf{F}$ & $\begin{array}{l}\text { Pulmonary TB, } \\
\text { rectal disease }\end{array}$ & Syphilis & Lues & - & - \\
\hline 117 & 707 & c. 26 years & $\mathbf{M}$ & Pulmonary TB & - & - & - & - \\
\hline 118 & 711 & 61 years & $\mathbf{M}$ & $\begin{array}{c}\mathrm{TB}, \\
\text { peritonitis }\end{array}$ & - & - & - & - \\
\hline 119 & 725 & 57 years & $\mathbf{M}$ & Pulmonary TB & - & - & - & - \\
\hline 120 & 728R & 76 years & $\mathbf{F}$ & $\begin{array}{c}\text { Pulmonary TB, } \\
\text { senility }\end{array}$ & - & - & - & + \\
\hline 121 & 739 & 40 years & $\mathbf{M}$ & $\begin{array}{c}\mathrm{TB}, \\
\text { diabetes mellitus }\end{array}$ & Pulmonary TB & Diabetes mellitus & - & + \\
\hline 122 & 740R & 18 years & $\mathbf{M}$ & $\begin{array}{c}\mathrm{TB}, \\
\text { peritonitis }\end{array}$ & - & - & - & - \\
\hline 123 & $745 R$ & c. 34 years & $\mathbf{F}$ & TB & - & - & - & - \\
\hline 124 & 752 & 71 years & $\mathbf{M}$ & $\begin{array}{c}\text { TB, } \\
\text { pneumonia }\end{array}$ & Pneumoconiosis & Myocarditis & Arteriosclerosis & + \\
\hline
\end{tabular}

- 178 - 


\begin{tabular}{|c|c|c|c|c|c|c|c|c|}
\hline \multirow{2}{*}{ No. } & \multirow{2}{*}{ Terry No. } & \multirow{2}{*}{$\begin{array}{c}\text { Age } \\
\text { at death }\end{array}$} & \multirow{2}{*}{ Sex } & \multicolumn{4}{|c|}{ Cause of death } & \multirow{2}{*}{$\begin{array}{l}\text { Included or not } \\
\text { into the evaluation }\end{array}$} \\
\hline & & & & MR & DC1 & DC2 & DC3 & \\
\hline 125 & 757 & 59 years & $\mathbf{M}$ & Pulmonary TB & - & - & - & + \\
\hline 126 & 760 & 18 years & M & TB spondylitis & - & - & - & - \\
\hline 127 & 761 & c. 81 years & $\mathbf{F}$ & Pulmonary TB & Pulmonary TB & Myocarditis & - & + \\
\hline 128 & 764 & c. 58 years & $\mathbf{M}$ & Pulmonary TB & - & - & - & - \\
\hline 129 & 770 & 65 years & $\mathbf{M}$ & Cardiorenal disease & Myocarditis & Pulmonary TB & - & - \\
\hline 130 & 771 & c. 49 years & $\mathbf{M}$ & Pulmonary TB & - & - & - & + \\
\hline 131 & 772 & c. 55 years & $\mathbf{M}$ & $\begin{array}{c}\text { Pulmonary TB, cirrhosis, } \\
\text { myocarditis }\end{array}$ & Myocarditis & - & - & - \\
\hline 132 & 776 & c. 40 years & M & Pulmonary TB & - & - & - & + \\
\hline 133 & 786 & 65 years & $\mathbf{M}$ & Pulmonary TB & - & - & - & + \\
\hline 134 & 799 & 38 years & $\mathbf{M}$ & TB & - & - & - & + \\
\hline 135 & 802 & c. 36 years & $\mathbf{M}$ & Pulmonary TB & - & - & - & - \\
\hline 136 & 810 & c. 54 years & $\mathbf{M}$ & TB & - & - & - & - \\
\hline 137 & 820R & 52 years & M & Pulmonary TB & - & - & - & + \\
\hline 138 & 821 & 25 years & M & Pulmonary TB & - & - & - & - \\
\hline
\end{tabular}




\begin{tabular}{|c|c|c|c|c|c|c|c|c|}
\hline No. & Terry No. & $\begin{array}{c}\text { Age } \\
\text { at death }\end{array}$ & Sex & \multicolumn{4}{|c|}{ Cause of death } & $\begin{array}{l}\text { Included or not } \\
\text { into the evaluation }\end{array}$ \\
\hline 139 & 822 & 16 years & $\mathbf{F}$ & Pulmonary TB & - & - & - & + \\
\hline 140 & 826 & 38 years & м & Pulmonary TB & - & - & - & - \\
\hline 141 & 828 & 46 years & $\mathbf{M}$ & $\mathbf{T B}$ & - & - & - & + \\
\hline 143 & 844 & 26 years & $\mathbf{F}$ & Pulmonary TB & - & - & - & + \\
\hline 144 & 846 & 48 years & $\mathbf{M}$ & Pulmonary TB & - & - & - & + \\
\hline 145 & 850 & 23 years & $\mathbf{M}$ & Pulmonary TB & - & - & - & - \\
\hline 148 & 876 & c. 22 years & $\mathbf{M}$ & Pulmonary TB & - & - & - & + \\
\hline 149 & 885 & 26 years & $\mathbf{M}$ & Pulmonary TB & - & - & - & - \\
\hline 150 & 886 & c. 23 years & $\mathbf{F}$ & Pulmonary TB & - & - & - & - \\
\hline 151 & 892 & c. 59 years & $\mathbf{M}$ & Heart disease & Emphysema & Pulmonary TB & - & + \\
\hline 152 & 895 & 47 years & $\mathbf{M}$ & TB & - & - & - & + \\
\hline
\end{tabular}

$-180-$ 


\begin{tabular}{|c|c|c|c|c|c|c|c|c|}
\hline \multirow{2}{*}{ No. } & \multirow{2}{*}{ Terry No. } & \multirow{2}{*}{$\begin{array}{c}\text { Age } \\
\text { at death }\end{array}$} & \multirow{2}{*}{ Sex } & \multicolumn{4}{|c|}{ Cause of death } & \multirow{2}{*}{$\begin{array}{l}\text { Included or not } \\
\text { into the evaluation }\end{array}$} \\
\hline & & & & MR & DC1 & DC2 & DC3 & \\
\hline 153 & 896RR & 26 years & $\mathbf{F}$ & Pulmonary TB & - & - & - & + \\
\hline 154 & 897 & 43 years & $\mathbf{M}$ & Pulmonary TB & Pulmonary TB & Myocarditis & - & + \\
\hline 155 & 902 & c. 36 years & $\mathbf{M}$ & Pulmonary TB & - & - & - & + \\
\hline 156 & 905 & 31 years & $\mathbf{M}$ & Pulmonary TB & - & - & - & - \\
\hline 157 & 907 & 41 years & $\mathbf{F}$ & Pulmonary TB & - & - & - & + \\
\hline 158 & 914 & 30 years & $\mathbf{M}$ & TB & - & - & - & + \\
\hline 159 & 915 & 27 years & $\mathbf{M}$ & Pulmonary TB & - & - & - & + \\
\hline 160 & 918 & c. 40 years & $\mathbf{M}$ & $\begin{array}{c}\mathrm{TB}, \\
\text { meningitis }\end{array}$ & - & - & - & - \\
\hline 161 & 925 & c. 71 years & $\mathbf{F}$ & $\mathbf{T B}$ & Pulmonary TB & Nephritis & Myocarditis & - \\
\hline 162 & 929 & c. 20 years & $\mathbf{F}$ & Pulmonary TB & - & - & - & - \\
\hline 163 & 932 & 27 years & $\mathbf{M}$ & Pulmonary TB & - & - & - & + \\
\hline 164 & 933R & 40 years & $\mathbf{M}$ & $\begin{array}{c}\text { Probable TB, } \\
\text { ascites }\end{array}$ & TB peritonitis & - & - & + \\
\hline 165 & 936 & 56 years & $\mathbf{M}$ & Pulmonary TB & - & - & - & + \\
\hline 166 & 940 & 24 years & $\mathbf{M}$ & TB & - & - & - & - \\
\hline
\end{tabular}




\begin{tabular}{|c|c|c|c|c|c|c|c|c|}
\hline \multirow{2}{*}{ No. } & \multirow{2}{*}{ Terry No. } & \multirow{2}{*}{$\begin{array}{c}\text { Age } \\
\text { at death }\end{array}$} & \multirow{2}{*}{ Sex } & \multicolumn{4}{|c|}{ Cause of death } & \multirow{2}{*}{$\begin{array}{l}\text { Included or not } \\
\text { into the evaluation }\end{array}$} \\
\hline & & & & MR & DC1 & DC2 & DC3 & \\
\hline 167 & 950 & 59 years & $\mathbf{M}$ & Pulmonary TB & - & - & - & + \\
\hline 168 & 952 & 41 years & $\mathbf{F}$ & TB & - & - & - & + \\
\hline 169 & 955 & 26 years & M & Pulmonary TB & - & - & - & + \\
\hline 170 & 957 & 56 years & $\mathbf{F}$ & Chest contusion & Pulmonary TB & - & - & + \\
\hline 171 & 960 & 17 years & $\mathbf{M}$ & Pulmonary TB & - & - & - & - \\
\hline 172 & 970 & 21 years & $\mathbf{F}$ & Pulmonary TB & - & - & - & - \\
\hline 173 & 975 & 60 years & $\mathbf{M}$ & $\begin{array}{c}\mathrm{TB}, \\
\text { cardiac decompensation }\end{array}$ & Myocarditis & - & - & + \\
\hline 174 & 980 & 63 years & $\mathbf{M}$ & Pulmonary TB & - & - & - & - \\
\hline 175 & 981 & 31 years & $\mathbf{M}$ & TB & - & - & - & - \\
\hline 176 & 987 & 23 years & $\mathbf{M}$ & Pulmonary TB & - & - & - & + \\
\hline 177 & 1002 & 50 years & $\mathbf{M}$ & Pulmonary TB & - & - & - & + \\
\hline 178 & 1005 & 52 years & $\mathbf{M}$ & Pulmonary TB & - & - & - & + \\
\hline 179 & $1007 R$ & 39 years & M & Pulmonary TB & - & - & - & - \\
\hline 180 & 1013 & c. 30 years & $\mathbf{M}$ & Pulmonary TB & - & - & - & + \\
\hline
\end{tabular}




\begin{tabular}{|c|c|c|c|c|c|c|c|c|}
\hline \multirow{2}{*}{ No. } & \multirow{2}{*}{ Terry No. } & \multirow{2}{*}{$\begin{array}{c}\text { Age } \\
\text { at death }\end{array}$} & \multirow{2}{*}{ Sex } & \multicolumn{4}{|c|}{ Cause of death } & \multirow{2}{*}{$\begin{array}{l}\text { Included or not } \\
\text { into the evaluation }\end{array}$} \\
\hline & & & & MR & DC1 & DC2 & DC3 & \\
\hline 181 & 1018 & 30 years & $\mathbf{M}$ & Pulmonary TB & - & - & - & + \\
\hline 182 & 1020 & 24 years & M & Pulmonary TB & - & - & - & + \\
\hline 183 & 1027 & c. 41 years & $\mathbf{F}$ & Pulmonary TB & - & - & - & + \\
\hline 184 & 1030 & 62 years & $\mathbf{M}$ & - & Pulmonary TB & - & - & + \\
\hline 185 & 1031 & 27 years & $\mathbf{M}$ & Pneumonia & Pulmonary TB & - & - & + \\
\hline 186 & 1033 & 26 years & M & Pulmonary TB & - & - & - & + \\
\hline 187 & 1034 & 48 years & $\mathbf{F}$ & Pulmonary TB & TB nephritis & - & - & + \\
\hline 188 & 1036 & 38 years & $\mathbf{M}$ & $\begin{array}{c}\text { Asthma, } \\
\text { heart disease }\end{array}$ & Pulmonary TB & - & - & + \\
\hline 189 & 1043 & 46 years & $\mathbf{M}$ & Pulmonary TB & - & - & - & + \\
\hline 190 & 1047 & c. 61 years & $\mathbf{M}$ & TB & - & - & - & + \\
\hline 191 & 1048 & 39 years & $\mathbf{M}$ & TB & - & - & - & + \\
\hline 192 & 1057 & 35 years & $\mathbf{M}$ & Pulmonary TB & - & - & - & + \\
\hline 193 & 1064 & 33 years & $\mathbf{F}$ & $\begin{array}{c}\text { TB, } \\
\text { pneumonia }\end{array}$ & - & - & - & - \\
\hline 194 & 1072 & 56 years & $\mathbf{M}$ & Pulmonary TB & - & - & - & + \\
\hline
\end{tabular}




\begin{tabular}{|c|c|c|c|c|c|c|c|c|}
\hline \multirow{2}{*}{ No. } & \multirow{2}{*}{ Terry No. } & \multirow{2}{*}{$\begin{array}{c}\text { Age } \\
\text { at death }\end{array}$} & \multirow{2}{*}{ Sex } & \multicolumn{4}{|c|}{ Cause of death } & \multirow{2}{*}{$\begin{array}{l}\text { Included or not } \\
\text { into the evaluation }\end{array}$} \\
\hline & & & & MR & DC1 & DC2 & DC3 & \\
\hline 195 & 1076 & 25 years & $\mathbf{F}$ & Pulmonary TB & Pulmonary TB & Pulmonary oedema & - & + \\
\hline 196 & 1086 & 45 years & M & Pulmonary TB & - & - & - & + \\
\hline 197 & 1093 & 29 years & $\mathbf{M}$ & Pulmonary TB & - & - & - & + \\
\hline 198 & 1095 & 61 years & $\mathbf{M}$ & Pulmonary TB & - & - & - & + \\
\hline 199 & 1096R & 40 years & $\mathbf{M}$ & Pulmonary TB & - & - & - & + \\
\hline 200 & 1105 & 22 years & $\mathbf{F}$ & Pulmonary TB & - & - & - & + \\
\hline 201 & 1106 & 28 years & $\mathbf{M}$ & $\begin{array}{l}\text { TB spondylitis, } \\
\text { heart disease }\end{array}$ & TB spondylitis & - & - & + \\
\hline 202 & 1113 & 29 years & M & Pulmonary TB & Myocarditis & - & - & + \\
\hline 203 & 1122 & 29 years & $\mathbf{F}$ & Pulmonary TB & - & - & - & + \\
\hline 204 & $1124 R$ & 49 years & $\mathbf{F}$ & Pulmonary TB & - & - & - & + \\
\hline 205 & 1129 & 50 years & $\mathbf{F}$ & Pulmonary TB & - & - & - & + \\
\hline 206 & 1132 & 67 years & M & Pulmonary TB & - & - & - & + \\
\hline 207 & 1147R & 32 years & $\mathbf{M}$ & Pleural expansion & $\begin{array}{l}\text { Possible TB, } \\
\text { pleural effusion }\end{array}$ & - & - & + \\
\hline 208 & 1149R & c. 44 years & $\mathbf{M}$ & Pulmonary TB & - & - & - & - \\
\hline
\end{tabular}

- 184 - 


\begin{tabular}{|c|c|c|c|c|c|c|c|c|}
\hline \multirow{2}{*}{ No. } & \multirow{2}{*}{ Terry No. } & \multirow{2}{*}{$\begin{array}{c}\text { Age } \\
\text { at death }\end{array}$} & \multirow{2}{*}{ Sex } & \multicolumn{4}{|c|}{ Cause of death } & \multirow{2}{*}{$\begin{array}{l}\text { Included or not } \\
\text { into the evaluation }\end{array}$} \\
\hline & & & & MR & DC1 & DC2 & DC3 & \\
\hline 209 & 1156 & c. 56 years & $\mathbf{M}$ & Pulmonary TB & - & - & - & + \\
\hline 210 & 1157 & 40 years & $\mathbf{M}$ & Pulmonary TB & - & - & - & + \\
\hline 211 & 1159 & 26 years & $\mathbf{M}$ & Pulmonary TB & - & - & - & + \\
\hline 212 & 1165 & c. 26 years & $\mathbf{M}$ & Pulmonary TB & - & - & - & + \\
\hline 213 & 1168 & 34 years & $\mathbf{M}$ & Pulmonary TB & - & - & - & - \\
\hline 214 & 1169 & 28 years & M & Pulmonary TB & - & - & - & + \\
\hline 215 & 1173 & 38 years & $\mathbf{F}$ & Pulmonary TB & - & - & - & + \\
\hline 216 & 1183 & c. 21 years & M & Pneumonia & Pulmonary TB & Pleurisy & - & + \\
\hline 217 & 1185 & 33 years & $\mathbf{M}$ & Pulmonary TB & $\begin{array}{c}\text { Pulmonary } \\
\text { haemorrhage }\end{array}$ & Pulmonary TB & - & + \\
\hline 218 & 1187 & 21 years & $\mathbf{M}$ & Pulmonary TB & - & - & - & + \\
\hline 219 & 1190 & c. 64 years & $\mathbf{M}$ & Pulmonary TB & - & - & - & + \\
\hline 220 & 1205 & 38 years & M & Pulmonary TB & - & - & - & + \\
\hline 221 & 1210 & 46 years & $\mathbf{F}$ & TB & Pulmonary TB & - & - & + \\
\hline 222 & 1215 & 25 years & $\mathbf{F}$ & Pulmonary TB & Pulmonary TB & Child birth & - & + \\
\hline
\end{tabular}

$-185-$ 


\begin{tabular}{|c|c|c|c|c|c|c|c|c|}
\hline \multirow{2}{*}{ No. } & \multirow{2}{*}{ Terry No. } & \multirow{2}{*}{$\begin{array}{c}\text { Age } \\
\text { at death }\end{array}$} & \multirow{2}{*}{ Sex } & \multicolumn{4}{|c|}{ Cause of death } & \multirow{2}{*}{$\begin{array}{l}\text { Included or not } \\
\text { into the evaluation }\end{array}$} \\
\hline & & & & MR & DC1 & DC2 & DC3 & \\
\hline 223 & 1222 & 28 years & $\mathbf{F}$ & Pulmonary TB & - & - & - & + \\
\hline 224 & 1226 & c. 48 years & M & Pulmonary TB & - & - & - & + \\
\hline 225 & 1230 & 53 years & $\mathbf{M}$ & $\begin{array}{l}\text { Cysto-urethro-rectocele } \\
\text { with ascites }\end{array}$ & Pulmonary TB & Pneumothorax & $\begin{array}{l}\text { Cirrhosis, } \\
\text { myocarditis }\end{array}$ & + \\
\hline 226 & 1236 & 36 years & $\mathbf{F}$ & TB & - & - & - & + \\
\hline 227 & 1247 & 53 years & $\mathbf{M}$ & TB & - & - & - & + \\
\hline 228 & 1249R & 40 years & $\mathbf{F}$ & - & Pulmonary TB & - & - & + \\
\hline 229 & 1255 & 39 years & $\mathbf{M}$ & TB sinusitis & TB of the hips & Delirium tremens & - & + \\
\hline 230 & $1263 R$ & 53 years & M & Pulmonary TB & - & - & - & + \\
\hline 231 & 1264 & 45 years & $\mathbf{M}$ & Pulmonary TB & - & - & - & + \\
\hline 232 & 1266R & c. 77 years & $\mathbf{M}$ & $\begin{array}{l}\text { Senility, } \\
\text { lues }\end{array}$ & Pulmonary TB & Syphilis & $\begin{array}{l}\text { Parkinson's disease, } \\
\text { arteriosclerosis }\end{array}$ & + \\
\hline 233 & 1275 & c. 59 years & $\mathbf{M}$ & $\begin{array}{l}\text { Pulmonary TB, } \\
\text { myocarditis }\end{array}$ & Pneumonia & Arteriosclerosis & - & + \\
\hline 234 & 1276 & 82 years & $\mathbf{M}$ & Pulmonary TB & - & - & - & - \\
\hline 235 & 1278 & 45 years & M & Pulmonary TB & - & - & - & + \\
\hline 236 & 1282 & 61 years & M & Myocarditis & Pulmonary TB & Myocarditis & - & + \\
\hline
\end{tabular}




\begin{tabular}{|c|c|c|c|c|c|c|c|c|}
\hline \multirow{2}{*}{ No. } & \multirow{2}{*}{ Terry No. } & \multirow{2}{*}{$\begin{array}{c}\text { Age } \\
\text { at death }\end{array}$} & \multirow{2}{*}{ Sex } & \multicolumn{4}{|c|}{ Cause of death } & \multirow{2}{*}{$\begin{array}{l}\text { Included or not } \\
\text { into the evaluation }\end{array}$} \\
\hline & & & & MR & DC1 & DC2 & DC3 & \\
\hline 237 & 1285 & 36 years & $\mathbf{M}$ & TB & - & - & - & + \\
\hline 238 & 1287 & 24 years & $\mathbf{F}$ & Pulmonary TB & - & - & - & + \\
\hline 239 & $1289 R$ & 28 years & $\mathbf{F}$ & Nephritis & TB nephritis & - & - & - \\
\hline 240 & 1293 & 51 years & $\mathbf{M}$ & Miliary TB & - & - & - & - \\
\hline 241 & 1300 & 28 years & $\mathbf{M}$ & TB & - & - & - & + \\
\hline 242 & $1304 R$ & 58 years & M & Pulmonary TB & Pulmonary TB & Pericardial effusion & - & + \\
\hline 243 & 1306 & 36 years & $\mathbf{F}$ & TB & - & - & - & - \\
\hline 244 & 1309 & 36 years & $\mathbf{M}$ & Neurological condition & TB spondylitis & Nephritis & $\begin{array}{c}\text { Paralysis of the lower } \\
\text { extremities }\end{array}$ & + \\
\hline 245 & 1313 & 63 years & $\mathbf{M}$ & TB & Syphilis & Pulmonary TB & $\begin{array}{l}\text { Myocarditis, } \\
\text { arteriosclerosis }\end{array}$ & + \\
\hline 246 & 1315 & 43 years & $\mathbf{M}$ & TB & - & - & - & + \\
\hline 247 & 1316R & c. 95 years & $\mathbf{F}$ & $\begin{array}{c}\text { Hand \& knee TB, } \\
\text { senility }\end{array}$ & Pneumonia & $\begin{array}{c}\text { Heart disease, } \\
\text { nephritis }\end{array}$ & - & - \\
\hline 248 & 1318 & 43 years & M & Pulmonary TB & - & - & - & + \\
\hline 249 & 1319 & c. 36 years & $\mathbf{M}$ & Pulmonary TB & Pulmonary TB & Syphilis & - & + \\
\hline 250 & 1322 & 34 years & $\mathbf{M}$ & Pulmonary TB & - & - & - & + \\
\hline
\end{tabular}

- 187 - 


\begin{tabular}{|c|c|c|c|c|c|c|c|c|}
\hline \multirow{2}{*}{ No. } & \multirow{2}{*}{ Terry No. } & \multirow{2}{*}{$\begin{array}{c}\text { Age } \\
\text { at death }\end{array}$} & \multirow{2}{*}{ Sex } & \multicolumn{4}{|c|}{ Cause of death } & \multirow{2}{*}{$\begin{array}{l}\text { Included or not } \\
\text { into the evaluation }\end{array}$} \\
\hline & & & & MR & DC1 & DC2 & DC3 & \\
\hline 251 & 1331 & 41 years & $\mathbf{M}$ & Pulmonary TB & - & - & - & + \\
\hline 252 & 1337RR & 35 years & $\mathbf{F}$ & Psychosis & Pulmonary TB & Brain syndrome & - & + \\
\hline 253 & 1346 & 73 years & M & Lung abscess & Pulmonary TB & - & - & + \\
\hline 254 & 1351 & 23 years & $\mathbf{F}$ & Pulmonary TB & - & - & - & - \\
\hline 255 & 1352 & 28 years & $\mathbf{M}$ & Pulmonary TB & - & - & - & + \\
\hline 256 & 1356 & 35 years & $\mathbf{F}$ & Pulmonary TB & - & - & - & - \\
\hline 257 & 1359 & 52 years & $\mathbf{M}$ & $\begin{array}{l}\text { Bronchitis, } \\
\text { alcoholism }\end{array}$ & Pulmonary TB & - & - & + \\
\hline 258 & 1361 & 52 years & $\mathbf{M}$ & TB meningitis & - & - & - & - \\
\hline 259 & 1362 & 36 years & $\mathbf{M}$ & Pulmonary TB & - & - & - & + \\
\hline 260 & 1363 & 24 years & $\mathbf{F}$ & Pulmonary TB & - & - & - & - \\
\hline 261 & 1367 & 32 years & $\mathbf{M}$ & Pulmonary TB & - & - & - & + \\
\hline 262 & 1369 & 52 years & $\mathbf{M}$ & Pulmonary TB & - & - & - & + \\
\hline 263 & 1377 & c. 52 years & $\mathbf{F}$ & Haemorrhage & $\begin{array}{l}\text { Haemorrhage } \\
\text { into the right ventricle }\end{array}$ & TB meningitis & - & + \\
\hline 264 & 1379 & c. 54 years & M & Myocarditis & Myocarditis & Pulmonary TB & - & + \\
\hline
\end{tabular}




\begin{tabular}{|c|c|c|c|c|c|c|c|c|}
\hline \multirow{2}{*}{ No. } & \multirow{2}{*}{ Terry No. } & \multirow{2}{*}{$\begin{array}{c}\text { Age } \\
\text { at death }\end{array}$} & \multirow{2}{*}{ Sex } & \multicolumn{4}{|c|}{ Cause of death } & \multirow{2}{*}{$\begin{array}{l}\text { Included or not } \\
\text { into the evaluation }\end{array}$} \\
\hline & & & & MR & DC1 & DC2 & DC3 & \\
\hline 265 & 1388 & 29 years & $\mathbf{M}$ & Pulmonary TB & Pulmonary TB & TB peritonitis & Cardiac decompensation & + \\
\hline 266 & 1397 & 35 years & $\mathbf{M}$ & $\mathbf{T B}$ & - & - & - & + \\
\hline 267 & 1398 & 31 years & $\mathbf{M}$ & $\begin{array}{c}\text { TB, } \\
\text { meningitis }\end{array}$ & - & - & - & + \\
\hline 268 & 1401 & 47 years & $\mathbf{F}$ & Pulmonary TB & - & - & - & + \\
\hline 269 & 1406 & 63 years & $\mathbf{F}$ & TB & - & - & - & + \\
\hline 270 & 1407 & 44 years & $\mathbf{M}$ & TB & - & - & - & + \\
\hline 271 & 1419 & 54 years & $\mathbf{F}$ & Pulmonary TB & - & - & - & + \\
\hline 272 & $1422 R$ & c. 35 years & $\mathbf{M}$ & Pulmonary TB & - & - & - & + \\
\hline 273 & $1428 R$ & 71 years & $\mathbf{M}$ & Pulmonary TB & - & - & - & + \\
\hline 274 & $1434 R$ & 19 years & $\mathbf{F}$ & Pulmonary TB & - & - & - & + \\
\hline 275 & 1451 & 36 years & $\mathbf{M}$ & TB & - & - & - & + \\
\hline 276 & $1453 R$ & 58 years & $\mathbf{M}$ & Pulmonary TB & - & - & - & + \\
\hline 277 & 1455 & 68 years & $\mathbf{M}$ & TB & TB & Pneumonia & - & + \\
\hline 278 & 1458 & 37 years & $\mathbf{M}$ & Pulmonary TB & - & - & - & + \\
\hline
\end{tabular}




\begin{tabular}{|c|c|c|c|c|c|c|c|c|}
\hline \multirow{2}{*}{ No. } & \multirow{2}{*}{ Terry No. } & \multirow{2}{*}{$\begin{array}{c}\text { Age } \\
\text { at death }\end{array}$} & \multirow{2}{*}{ Sex } & \multicolumn{4}{|c|}{ Cause of death } & \multirow{2}{*}{$\begin{array}{l}\text { Included or not } \\
\text { into the evaluation }\end{array}$} \\
\hline & & & & MR & DC1 & DC2 & DC3 & \\
\hline 279 & 1468 & 41 years & $\mathbf{F}$ & $\begin{array}{c}\text { TB, } \\
\text { pneumonia }\end{array}$ & - & - & - & - \\
\hline 280 & 1469 & 42 years & $\mathbf{M}$ & $\begin{array}{c}\mathrm{TB}, \\
\text { diabetes mellitus }\end{array}$ & - & - & - & - \\
\hline 281 & 1476 & 51 years & $\mathbf{F}$ & $\begin{array}{l}\text { Pulmonary TB, } \\
\text { TB adenitis }\end{array}$ & - & - & - & + \\
\hline 282 & 1486R & 51 years & $\mathbf{M}$ & Diabetes mellitus & Pulmonary TB & Diabetes mellitus & - & - \\
\hline 283 & 1500 & 24 years & $\mathbf{F}$ & Pulmonary phthisis & Pulmonary TB & - & - & - \\
\hline 284 & 1503 & 22 years & $\mathbf{M}$ & Pulmonary TB & - & - & - & + \\
\hline 285 & 1506 & 26 years & $\mathbf{M}$ & Pulmonary TB & - & - & - & - \\
\hline 286 & 1507 & 23 years & $\mathbf{F}$ & $\begin{array}{l}\text { Pulmonary TB, } \\
\text { heart disease }\end{array}$ & - & - & - & + \\
\hline 287 & 1508 & 55 years & $\mathbf{M}$ & Pulmonary TB & - & - & - & - \\
\hline 288 & 1521 & 54 years & $\mathbf{M}$ & Pulmonary TB & - & - & - & + \\
\hline 289 & 1529 & c. 28 years & $\mathbf{M}$ & TB & Pulmonary TB & - & - & - \\
\hline 290 & 1531 & 40 years & M & Pulmonary TB & - & - & - & + \\
\hline 291 & 1533 & 45 years & $\mathbf{M}$ & Pulmonary TB & - & - & - & + \\
\hline 292 & 1536 & 71 years & $\mathbf{F}$ & Pulmonary TB & - & - & - & + \\
\hline
\end{tabular}

- 190 - 


\begin{tabular}{|c|c|c|c|c|c|c|c|c|}
\hline \multirow{2}{*}{ No. } & \multirow{2}{*}{ Terry No. } & \multirow{2}{*}{$\begin{array}{c}\text { Age } \\
\text { at death }\end{array}$} & \multirow{2}{*}{ Sex } & \multicolumn{4}{|c|}{ Cause of death } & \multirow{2}{*}{$\begin{array}{l}\text { Included or not } \\
\text { into the evaluation }\end{array}$} \\
\hline & & & & MR & DC1 & DC2 & DC3 & \\
\hline 293 & 1539 & 23 years & $\mathbf{M}$ & Pulmonary TB & - & - & - & + \\
\hline 294 & 1544 & c. 24 years & $\mathbf{F}$ & TB & Pulmonary TB & - & - & + \\
\hline 295 & 1551 & 25 years & $\mathbf{F}$ & $?$ & Salpingitis & TB peritonitis & - & + \\
\hline 296 & 1553 & 30 years & $\mathbf{F}$ & $\begin{array}{l}\text { Possible TB, } \\
\text { heart disease }\end{array}$ & Nephritis & - & - & + \\
\hline 297 & 1555 & 41 years & $\mathbf{F}$ & Pulmonary TB & - & - & - & + \\
\hline 298 & 1562 & 46 years & $\mathbf{F}$ & Pulmonary TB & - & - & - & + \\
\hline 299 & 1568 & c. 62 years & $\mathbf{F}$ & TB & Pulmonary TB & - & - & + \\
\hline 300 & 1572 & c. 44 years & $\mathbf{F}$ & Pulmonary TB & - & - & - & + \\
\hline 301 & 1576 & 69 years & $\mathbf{F}$ & Pulmonary TB & Pulmonary TB & Schizophrenic reaction & - & + \\
\hline 302 & 1629 & 80 years & $\mathbf{F}$ & Pneumonia & Pulmonary TB & - & - & + \\
\hline
\end{tabular}


Supplementary table 2: Basic biographic data of specimens in the NTB group $(\mathrm{N}=302)$.


$+=$ included into the evaluation considering TBM-related endocranial alterations; $-=$ excluded from the evaluation considering TBM-related endocranial lesions).

\begin{tabular}{|c|c|c|c|c|c|c|c|c|}
\hline \multirow{2}{*}{ No. } & \multirow{2}{*}{ Terry No. } & \multirow{2}{*}{$\begin{array}{c}\text { Age } \\
\text { at death }\end{array}$} & \multirow{2}{*}{ Sex } & \multicolumn{4}{|c|}{ Cause of death } & \multirow{2}{*}{$\begin{array}{l}\text { Included or not } \\
\text { into the evaluation }\end{array}$} \\
\hline & & & & MR & DC1 & DC2 & DC3 & \\
\hline 1 & $4 R$ & 40 years & $\mathbf{M}$ & Antral carcinoma & - & $\begin{array}{l}\text { Metastasis } \\
\text { of the left orbit }\end{array}$ & - & + \\
\hline 2 & 5 & 67 years & $\mathbf{M}$ & Cardiac decompensation & $\begin{array}{l}\text { Arteriosclerotic } \\
\text { heart disease }\end{array}$ & $\begin{array}{c}\text { Congestive } \\
\text { heart failure }\end{array}$ & - & - \\
\hline 3 & $12 R$ & 46 years & $\mathbf{F}$ & Rectal cancer & - & - & - & + \\
\hline 4 & 15RRR & c. 72 years & $\mathbf{F}$ & Myocardial infarction & - & - & - & - \\
\hline 5 & 19R & c. 69 years & $\mathbf{F}$ & $\begin{array}{l}\text { Hypertensive } \\
\text { heart disease }\end{array}$ & $\begin{array}{l}\text { Hypertensive } \\
\text { heart disease }\end{array}$ & - & - & + \\
\hline 6 & 25 & c. 65 years & $\mathbf{F}$ & - & - & - & - & + \\
\hline 7 & $25 R$ & c. 43 years & $\mathbf{F}$ & Alcoholism & Pulmonary oedema & Cerebral oedema & Alcoholism & + \\
\hline 8 & 31R & c. 38 years & $\mathbf{M}$ & Stab wound & Cardiac thrombosis & $\begin{array}{l}\text { Septic stab wound } \\
\text { in the right arm }\end{array}$ & - & + \\
\hline 9 & $44 R$ & c. 72 years & $\mathbf{F}$ & Cerebral vascular disease & $\begin{array}{c}\text { Cerebral } \\
\text { vascular accident }\end{array}$ & $\begin{array}{l}\text { Hypertensive } \\
\text { heart disease }\end{array}$ & - & + \\
\hline 10 & 46R & c. 67 years & $\mathbf{F}$ & Accident & Pneumonia & $\begin{array}{c}\text { Subdural } \\
\text { haemorrhage from fall }\end{array}$ & - & + \\
\hline 11 & $47 R$ & 78 years & $\mathbf{F}$ & Pneumonia & - & - & - & + \\
\hline 12 & 53R & c. 60 years & $\mathbf{F}$ & Myocarditis & - & Arteriosclerosis & - & - \\
\hline
\end{tabular}




\begin{tabular}{|c|c|c|c|c|c|c|c|c|}
\hline \multirow{2}{*}{ No. } & \multirow{2}{*}{ Terry No. } & \multirow{2}{*}{$\begin{array}{c}\text { Age } \\
\text { at death }\end{array}$} & \multirow{2}{*}{ Sex } & \multicolumn{4}{|c|}{ Cause of death } & \multirow{2}{*}{$\begin{array}{l}\text { Included or not } \\
\text { into the evaluation }\end{array}$} \\
\hline & & & & MR & DC1 & DC2 & DC3 & \\
\hline 13 & $58 R$ & c. 70 years & $\mathbf{F}$ & Cardiorenal disease & $\begin{array}{l}\text { Hypertensive } \\
\text { heart disease }\end{array}$ & - & - & + \\
\hline 14 & 61 & c. 74 years & $\mathbf{M}$ & $\begin{array}{l}\text { Senility, } \\
\text { hypertrophic arthritis }\end{array}$ & Myocarditis & Hypertrophic arthritis & - & - \\
\hline 15 & 62RR & c. 38 years & $\mathbf{M}$ & - & - & - & - & + \\
\hline 16 & $64 R$ & 57 years & $\mathbf{F}$ & Cervical cancer & $\begin{array}{l}\text { Cancerous cervix } \\
\text { with metastasis }\end{array}$ & - & - & + \\
\hline 17 & $67 R$ & 74 years & $\mathbf{F}$ & $\begin{array}{l}\text { Arteriosclerotic } \\
\text { heart disease }\end{array}$ & $\begin{array}{l}\text { Hypertensive } \\
\text { heart disease }\end{array}$ & - & - & - \\
\hline 18 & 69 & 75 years & $\mathbf{F}$ & $\begin{array}{l}\text { Breast cancer, } \\
\text { pneumonia }\end{array}$ & - & Pneumonia & - & + \\
\hline 19 & $75 R$ & c. 70 years & $\mathbf{F}$ & - & Nephritis & $\begin{array}{l}\text { Arteriosclerotic } \\
\text { heart disease }\end{array}$ & - & - \\
\hline 20 & $76 R$ & c. 62 years & $\mathbf{F}$ & Brain oedema & Myocarditis & Pleural effusion & - & + \\
\hline 21 & $79 R$ & c. 52 years & $\mathbf{F}$ & Nephritis & Nephritis & - & - & + \\
\hline 22 & $86 R$ & 71 years & $\mathbf{F}$ & $\begin{array}{c}\text { Congestive } \\
\text { heart disease }\end{array}$ & $\begin{array}{l}\text { Arteriosclerotic } \\
\text { heart disease }\end{array}$ & - & - & - \\
\hline 23 & 92R & 67 years & $\mathbf{F}$ & Cerebral thrombosis & - & - & - & - \\
\hline 24 & $96 R$ & c. 83 years & $\mathbf{F}$ & Cerebral arteriosclerosis & - & - & - & - \\
\hline 25 & 104RR & c. 80 years & $\mathbf{F}$ & - & $\begin{array}{c}\text { Cerebral } \\
\text { vascular accident }\end{array}$ & $\begin{array}{l}\text { Arteriosclerotic } \\
\text { heart disease }\end{array}$ & - & + \\
\hline 26 & $105 R$ & 81 years & $\mathbf{F}$ & Heart failure & - & Arteriosclerosis & - & + \\
\hline
\end{tabular}




\begin{tabular}{|c|c|c|c|c|c|c|c|c|}
\hline \multirow{2}{*}{ No. } & \multirow{2}{*}{ Terry No. } & \multirow{2}{*}{$\begin{array}{c}\text { Age } \\
\text { at death }\end{array}$} & \multirow{2}{*}{ Sex } & \multicolumn{4}{|c|}{ Cause of death } & \multirow{2}{*}{$\begin{array}{l}\text { Included or not } \\
\text { into the evaluation }\end{array}$} \\
\hline & & & & MR & DC1 & DC2 & DC3 & \\
\hline 27 & $112 R$ & c. 59 years & $\mathbf{F}$ & Rectal cancer & - & - & - & + \\
\hline 28 & $117 R$ & 81 years & $\mathbf{F}$ & Pneumonia & - & $\begin{array}{c}\text { Fractured } \\
\text { neck \& femur }\end{array}$ & Senile psychosis & - \\
\hline 29 & $124 R$ & 61 years & $\mathbf{F}$ & $\begin{array}{c}\text { Congestive } \\
\text { heart failure }\end{array}$ & $\begin{array}{l}\text { Hypertensive } \\
\text { heart disease }\end{array}$ & Nephritis & - & + \\
\hline 30 & $127 R$ & c. 72 years & $\mathbf{F}$ & Scleroderma & - & - & - & + \\
\hline 31 & $132 R$ & 39 years & $\mathbf{F}$ & Heart disease & - & Nephritis & - & + \\
\hline 32 & 134 & 53 years & $\mathbf{F}$ & Pneumonia & Pneumonia & - & - & + \\
\hline 33 & $135 R$ & c. 43 years & $\mathbf{F}$ & Heart failure & Coronary thrombosis & - & $\begin{array}{c}\text { psychosis, } \\
\text { Dementia precox }\end{array}$ & + \\
\hline 34 & 140RR & c. 56 years & $\mathbf{F}$ & Pneumonia & - & - & - & + \\
\hline 35 & $141 R$ & c. 83 years & $\mathbf{F}$ & Cerebral accident & $\begin{array}{l}\text { Arteriosclerotic } \\
\text { heart disease }\end{array}$ & - & - & + \\
\hline 36 & $142 R$ & c. 82 years & $\mathbf{F}$ & Pneumonia & - & Fractured hip & Senility & + \\
\hline 37 & 143 & 58 years & $\mathbf{M}$ & Cardiac decompensation & $\begin{array}{l}\text { Hypertensive } \\
\text { heart disease }\end{array}$ & - & - & - \\
\hline 38 & 149R & 70 years & $\mathbf{F}$ & Nephritis & Uraemia & Arteriosclerosis & $\begin{array}{l}\text { Hypertensive heart } \\
\text { disease, psychosis }\end{array}$ & + \\
\hline 39 & 159R & c. 68 years & $\mathbf{F}$ & Pneumonia & - & - & - & - \\
\hline 40 & 167 & 77 years & $\mathbf{M}$ & Cardiorenal disease & Myocarditis & Lues & - & + \\
\hline
\end{tabular}




\begin{tabular}{|c|c|c|c|c|c|c|c|c|}
\hline \multirow{2}{*}{ No. } & \multirow{2}{*}{ Terry No. } & \multirow{2}{*}{$\begin{array}{c}\text { Age } \\
\text { at death }\end{array}$} & \multirow{2}{*}{ Sex } & \multicolumn{4}{|c|}{ Cause of death } & \multirow{2}{*}{$\begin{array}{l}\text { Included or not } \\
\text { into the evaluation }\end{array}$} \\
\hline & & & & MR & DC1 & DC2 & DC3 & \\
\hline 41 & $177 R$ & c. 33 years & $\mathbf{F}$ & Haemorrhage & Left lung haemorrhage & - & - & + \\
\hline 42 & 178R & c. 60 years & $\mathbf{F}$ & $\begin{array}{l}\text { Hypertensive } \\
\text { encephalopathy }\end{array}$ & Cerebral thrombosis & Hypertension & - & + \\
\hline 43 & 179R & c. 76 years & $\mathbf{F}$ & Myocarditis & - & - & - & + \\
\hline 44 & 197R & 60 years & $\mathbf{F}$ & Breast carcinoma & - & - & - & + \\
\hline 45 & 199 & c. 70 years & $\mathbf{F}$ & Heart failure & Myocarditis & Senility & - & + \\
\hline 46 & 209 & 38 years & $\mathbf{M}$ & Pneumonia & - & - & - & + \\
\hline 47 & 213 & 29 years & $\mathbf{M}$ & Nephritis & Nephritis & Urethral fistulae & - & - \\
\hline 48 & 218 & 56 years & $\mathbf{F}$ & $\begin{array}{c}\text { Diabetes mellitus, } \\
\text { asthma }\end{array}$ & - & - & - & + \\
\hline 49 & 221 & c. 47 years & $\mathbf{M}$ & Intestinal obstruction & - & - & - & + \\
\hline $\mathbf{5 0}$ & 227 & 57 years & $\mathbf{M}$ & Syphilis & - & - & - & + \\
\hline 51 & 231 & c. 60 years & $\mathbf{M}$ & $\begin{array}{l}\text { Syphilis, } \\
\text { senility }\end{array}$ & Breast carcinoma & $\begin{array}{l}\text { Syphilis, } \\
\text { senility }\end{array}$ & - & + \\
\hline 52 & 237 & 70 years & $\mathbf{M}$ & $\begin{array}{l}\text { Hypopyon ulcer } \\
\text { of the eye }\end{array}$ & - & Pneumonia & - & + \\
\hline 53 & 239R & c. 50 years & $\mathbf{F}$ & - & Myocarditis & WMA & - & - \\
\hline 54 & 243R & c. 74 years & $\mathbf{F}$ & Diabetes mellitus & Myocardial infarction & Diabetes mellitus & - & + \\
\hline
\end{tabular}

- 195 - 


\begin{tabular}{|c|c|c|c|c|c|c|c|c|}
\hline \multirow{2}{*}{ No. } & \multirow{2}{*}{ Terry No. } & \multirow{2}{*}{$\begin{array}{c}\text { Age } \\
\text { at death }\end{array}$} & \multirow{2}{*}{ Sex } & \multicolumn{4}{|c|}{ Cause of death } & \multirow{2}{*}{$\begin{array}{l}\text { Included or not } \\
\text { into the evaluation }\end{array}$} \\
\hline & & & & MR & DC1 & DC2 & DC3 & \\
\hline 55 & 244 & c. 66 years & $\mathbf{M}$ & Autopsy & Nephritis & - & - & - \\
\hline 56 & 247R & 90 years & $\mathbf{F}$ & Myocardial failure & - & - & - & + \\
\hline 57 & 249R & 81 years & $\mathbf{F}$ & - & Cardiac dilatation & Myocarditis & - & + \\
\hline 58 & 259 & 52 years & $\mathbf{M}$ & Pneumonia & - & - & - & + \\
\hline 59 & 268 & 62 years & $\mathbf{M}$ & Senility & Myocarditis & - & - & + \\
\hline 60 & 272 & 49 years & $\mathbf{F}$ & $\begin{array}{c}\text { Cardiac decompensation, } \\
\text { nephritis }\end{array}$ & - & - & - & + \\
\hline 61 & 285 & 54 years & $\mathbf{M}$ & Pneumonia & - & - & - & + \\
\hline 62 & 289R & 47 years & $\mathbf{F}$ & Nephritis & - & Laennec's cirrhosis & - & - \\
\hline 63 & 290R & c. 65 years & $\mathbf{M}$ & - & - & - & - & - \\
\hline 64 & 293R & c. 54 years & $\mathbf{F}$ & Coronary occlusion & Coronary sclerosis & - & - & + \\
\hline 65 & 296R & 69 years & $\mathbf{M}$ & Myocarditis & - & - & - & + \\
\hline 66 & 298 & c. 53 years & $\mathbf{M}$ & - & - & - & - & + \\
\hline 67 & $306 R$ & c. 50 years & $\mathbf{F}$ & Hepatic cirrhosis & Brain oedema & Hepatic cirrhosis & - & + \\
\hline 68 & 312R & 64 years & $\mathbf{F}$ & Hepatic cirrhosis & - & - & - & - \\
\hline
\end{tabular}

- 196 - 


\begin{tabular}{|c|c|c|c|c|c|c|c|c|}
\hline \multirow{2}{*}{ No. } & \multirow{2}{*}{ Terry No. } & \multirow{2}{*}{$\begin{array}{l}\text { Age } \\
\text { at death }\end{array}$} & \multirow{2}{*}{ Sex } & \multicolumn{4}{|c|}{ Cause of death } & \multirow{2}{*}{$\begin{array}{l}\text { Included or not } \\
\text { into the evaluation }\end{array}$} \\
\hline & & & & MR & DC1 & DC2 & DC3 & \\
\hline 69 & 314 & 65 years & $\mathbf{M}$ & $\begin{array}{c}\text { Senility, } \\
\text { pneumonia }\end{array}$ & - & Myocarditis & - & + \\
\hline 70 & 317 & 84 years & $\mathbf{M}$ & Arteriosclerosis & - & - & - & + \\
\hline 71 & 338 & 65 years & $\mathbf{M}$ & $\begin{array}{c}\text { Pneumonia, } \\
\text { myocarditis, senility }\end{array}$ & - & - & - & + \\
\hline 72 & 339R & 67 years & $\mathbf{F}$ & Myocarditis & - & - & - & + \\
\hline 73 & 344R & 52 years & $\mathbf{F}$ & Pneumonia & Nephritis & - & - & + \\
\hline 74 & 347 & c. 73 years & $\mathbf{M}$ & Syphilis & - & Syphilis & - & + \\
\hline 75 & 348R & c. 28 years & $\mathbf{F}$ & Eclampsia & Nephritis & $\begin{array}{c}\text { Pregnancy } \\
\text { with eclampsia }\end{array}$ & - & + \\
\hline 76 & $349 R$ & c. 50 years & $\mathbf{F}$ & Pneumonia & - & - & - & - \\
\hline 77 & 360 & c. 46 years & $\mathbf{M}$ & $\begin{array}{c}\text { Knee sarcoma } \\
\text { with metastasis }\end{array}$ & - & - & - & - \\
\hline 78 & 363 & c. 65 years & $\mathbf{M}$ & $\begin{array}{c}\text { Cardiac condition, } \\
\text { senility }\end{array}$ & Myocarditis & $\begin{array}{c}\text { Apoplexy, } \\
\text { cerebral haemorrhage }\end{array}$ & - & - \\
\hline 79 & 369 & 76 years & $\mathbf{M}$ & $\begin{array}{c}\text { Fractured skull, } \\
\text { senility }\end{array}$ & - & Myocarditis & Nephritis & - \\
\hline 80 & 379 & 58 years & $\mathbf{M}$ & $\begin{array}{l}\text { Carcinoma of the } \\
\text { cervical glands }\end{array}$ & - & - & - & - \\
\hline 81 & 391 & 50 years & $\mathbf{M}$ & Gangrenous toes & Diabetes mellitus & - & - & - \\
\hline 82 & 393RR & c. 58 years & $\mathbf{F}$ & Accident & Pneumonia & Femur fracture & - & + \\
\hline
\end{tabular}

- 197 - 


\begin{tabular}{|c|c|c|c|c|c|c|c|c|}
\hline \multirow{2}{*}{ No. } & \multirow{2}{*}{ Terry No. } & \multirow{2}{*}{$\begin{array}{l}\text { Age } \\
\text { at death }\end{array}$} & \multirow{2}{*}{ Sex } & \multicolumn{4}{|c|}{ Cause of death } & \multirow{2}{*}{$\begin{array}{l}\text { Included or not } \\
\text { into the evaluation }\end{array}$} \\
\hline & & & & MR & DC1 & DC2 & DC3 & \\
\hline 83 & 397 & 60 years & $\mathbf{M}$ & Uraemia & Syphilis & Myocarditis & Nephritis & + \\
\hline 84 & 403 & 52 years & $\mathbf{M}$ & Cardiorenal disease & Myocarditis & Nephritis & - & + \\
\hline 85 & $405 R$ & c. 34 years & $\mathbf{F}$ & Hepatic abscess & - & - & - & - \\
\hline 86 & 408R & 74 years & $\mathbf{F}$ & Arteriosclerosis & - & - & - & - \\
\hline 87 & 418R & 75 years & $\mathbf{F}$ & $\begin{array}{c}\text { Congestive } \\
\text { heart disease }\end{array}$ & $\begin{array}{l}\text { Hypertensive } \\
\text { heart disease }\end{array}$ & Arteriosclerosis & - & - \\
\hline 88 & 422 & c. 20 years & $\mathbf{M}$ & Pneumonia & - & - & - & + \\
\hline 89 & 431 & 42 years & $\mathbf{M}$ & Cholecystectomy & - & Pneumonia & - & - \\
\hline 90 & 433 & c. 59 years & $\mathbf{M}$ & Railroad accident & - & - & - & - \\
\hline 91 & $437 R$ & c. 44 years & $\mathbf{F}$ & Pneumonia & - & - & - & + \\
\hline 92 & 438 & c. 86 years & $\mathbf{M}$ & - & - & - & - & + \\
\hline 93 & 445 & 66 years & $\mathbf{M}$ & $\begin{array}{c}\text { Ruptured gastric ulcer, } \\
\text { peritonitis }\end{array}$ & - & - & - & + \\
\hline 94 & 447 & 49 years & $\mathbf{M}$ & - & Nephritis & - & - & + \\
\hline 95 & 452 & c. 61 years & $\mathbf{M}$ & $\begin{array}{l}\text { Syphilis, } \\
\text { senility }\end{array}$ & Syphilis & Nephritis & - & + \\
\hline 96 & 453 & c. 42 years & $\mathbf{M}$ & Gangrenous bowel & $\begin{array}{c}\text { Strangulated } \\
\text { inguinal hernia }\end{array}$ & - & - & + \\
\hline
\end{tabular}




\begin{tabular}{|c|c|c|c|c|c|c|c|c|}
\hline \multirow{2}{*}{ No. } & \multirow{2}{*}{ Terry No. } & \multirow{2}{*}{$\begin{array}{c}\text { Age } \\
\text { at death }\end{array}$} & \multirow{2}{*}{ Sex } & \multicolumn{4}{|c|}{ Cause of death } & \multirow{2}{*}{$\begin{array}{l}\text { Included or not } \\
\text { into the evaluation }\end{array}$} \\
\hline & & & & MR & DC1 & DC2 & DC3 & \\
\hline 97 & 458 & 41 years & $\mathbf{M}$ & Haemorrhoids & Nephritis & - & - & + \\
\hline 98 & 463 & c. 50 years & $\mathbf{M}$ & $\begin{array}{l}\text { Myocarditis, } \\
\text { rheumatism }\end{array}$ & - & - & - & + \\
\hline 99 & 465 & 25 years & $\mathbf{M}$ & $\begin{array}{l}\text { Post-operative wound } \\
\text { of the chest }\end{array}$ & Aortic aneurysm & - & - & + \\
\hline 100 & 470 & c. 44 years & $\mathbf{M}$ & Cardiorenal disease & - & - & - & + \\
\hline 101 & 477 & 22 years & $\mathbf{M}$ & $\begin{array}{c}\text { Paralysis } \\
\text { of the lower extremities }\end{array}$ & $\begin{array}{c}\text { Toxaemia } \\
\text { from bed sores }\end{array}$ & $\begin{array}{l}\text { Septicaemia from } \\
\text { ulcers of the buttocks }\end{array}$ & - & + \\
\hline 102 & 483 & 44 years & $\mathbf{M}$ & Pneumonia & - & - & - & + \\
\hline 103 & $484 R$ & 73 years & $\mathbf{F}$ & - & Myocarditis & Arteriosclerosis & - & - \\
\hline 104 & 487 & c. 42 years & $\mathbf{F}$ & $\begin{array}{c}\text { Toxaemia } \\
\text { of pregnancy }\end{array}$ & Eclampsia & - & - & - \\
\hline 105 & 488 & c. 40 years & $\mathbf{F}$ & Hemiplegia & - & - & - & - \\
\hline 106 & 496 & 54 years & $\mathbf{M}$ & Pellagra & - & - & - & + \\
\hline 107 & 497 & c. 72 years & $\mathbf{M}$ & Nephritis & - & - & - & + \\
\hline 108 & 501 & 77 years & $\mathbf{M}$ & Valvular disease & Arteriosclerosis & - & - & - \\
\hline 109 & 506 & 48 years & $\mathbf{M}$ & Myocarditis & Asthma & Myocarditis & - & + \\
\hline 110 & 511 & 34 years & $\mathbf{F}$ & Syphilis & - & - & - & - \\
\hline
\end{tabular}




\begin{tabular}{|c|c|c|c|c|c|c|c|c|}
\hline \multirow{2}{*}{ No. } & \multirow{2}{*}{ Terry No. } & \multirow{2}{*}{$\begin{array}{l}\text { Age } \\
\text { at death }\end{array}$} & \multirow{2}{*}{ Sex } & \multicolumn{4}{|c|}{ Cause of death } & \multirow{2}{*}{$\begin{array}{l}\text { Included or not } \\
\text { into the evaluation }\end{array}$} \\
\hline & & & & MR & DC1 & DC2 & DC3 & \\
\hline 111 & 512 & c. 50 years & $\mathbf{F}$ & Mental condition & Myocarditis & Pneumonia & - & + \\
\hline 112 & 513RR & c. 38 years & $\mathbf{F}$ & - & $\begin{array}{l}\text { Haemorrhagic } \\
\text { pachyonychia }\end{array}$ & - & - & + \\
\hline 113 & 520 & c. 27 years & $\mathbf{F}$ & - & - & - & - & - \\
\hline 114 & $526 R$ & c. 78 years & $\mathbf{F}$ & $\begin{array}{l}\text { Arteriosclerotic } \\
\text { heart disease }\end{array}$ & - & - & - & - \\
\hline 115 & 527 & c. 54 years & $\mathbf{M}$ & Intestinal carcinoma & Diarrhoea & Enteritis & Myocarditis & + \\
\hline 116 & 528 & c. 64 years & $\mathbf{F}$ & Cerebral haemorrhage & Myocarditis & - & - & + \\
\hline 117 & 534 & c. 50 years & $\mathbf{M}$ & Stenosis & - & - & - & + \\
\hline 118 & 536 & 60 years & $\mathbf{M}$ & Cerebral haemorrhage & - & Arteriosclerosis & - & + \\
\hline 119 & 545 & 77 years & $\mathbf{M}$ & Heart disease & Myocarditis & Arteriosclerosis & - & + \\
\hline 120 & 552 & 72 years & $\mathbf{M}$ & Myocarditis & Nephritis & Myocarditis & - & + \\
\hline 121 & 573 & 59 years & $\mathbf{M}$ & Syphilis & - & - & - & + \\
\hline 122 & 582 & c. 49 years & $\mathbf{M}$ & Pneumonia & - & - & - & + \\
\hline 123 & 586 & 40 years & $\mathbf{F}$ & Cardiorenal disease & Nephritis & - & - & + \\
\hline 124 & 597 & c. 61 years & $\mathbf{M}$ & Myocarditis & $\begin{array}{c}\text { Valvular } \\
\text { heart disease }\end{array}$ & - & - & + \\
\hline
\end{tabular}




\begin{tabular}{|c|c|c|c|c|c|c|c|c|}
\hline \multirow{2}{*}{ No. } & \multirow{2}{*}{ Terry No. } & \multirow{2}{*}{$\begin{array}{c}\text { Age } \\
\text { at death }\end{array}$} & \multirow{2}{*}{ Sex } & \multicolumn{4}{|c|}{ Cause of death } & \multirow{2}{*}{$\begin{array}{l}\text { Included or not } \\
\text { into the evaluation }\end{array}$} \\
\hline & & & & MR & DC1 & DC2 & DC3 & \\
\hline 125 & 602 & 49 years & $\mathbf{M}$ & Pleurisy & Influenza & $\begin{array}{c}\text { Pulmonary infarct \& } \\
\text { abscess }\end{array}$ & $\begin{array}{c}\text { Pulmonary } \\
\text { haemorrhage }\end{array}$ & + \\
\hline 126 & 608 & 45 years & $\mathbf{M}$ & $\begin{array}{c}\text { Pneumonia, } \\
\text { mitral regurgitation }\end{array}$ & Myocarditis & Nephritis & - & + \\
\hline 127 & 615 & 35 years & $\mathbf{F}$ & Salpingectomy & Pneumonia & $\begin{array}{l}\text { Hysterectomy } \\
\text { for fibroid uterus }\end{array}$ & - & - \\
\hline 128 & $616 R$ & c. 55 years & $\mathbf{F}$ & Possible uraemia & $\begin{array}{l}\text { Hypertensive } \\
\text { heart disease }\end{array}$ & - & - & - \\
\hline 129 & 617 & 90 years & $\mathbf{F}$ & $\begin{array}{c}\text { Senility, } \\
\text { myocarditis }\end{array}$ & Myocarditis & - & - & - \\
\hline 130 & $617 R$ & 43 years & $\mathbf{F}$ & Pneumonia & - & Encephalic malacia & - & + \\
\hline 131 & 619 & 31 years & $\mathbf{M}$ & Pneumonia & Pneumonia & - & - & - \\
\hline 132 & $627 R$ & c. 27 years & $\mathbf{F}$ & Poisoning & Arsenical poisoning & Neosalvarsan & - & + \\
\hline 133 & 629 & c. 72 years & $\mathbf{M}$ & $\begin{array}{l}\text { Pneumonia, } \\
\text { senility }\end{array}$ & - & Hemiplegia & - & + \\
\hline 134 & 633 & 78 years & $\mathbf{M}$ & Myocraditis & Myocarditis & Arteriosclerosis & - & - \\
\hline 135 & 636 & 51 years & $\mathbf{M}$ & Cardiac condition & Myocarditis & Bronchitis & - & + \\
\hline 136 & 643 & c. 60 years & $\mathbf{M}$ & Cardiorenal disease & Myocarditis & Nephritis & - & - \\
\hline 137 & 648 & c. 63 years & $\mathbf{M}$ & $\begin{array}{c}\text { Erysipelas, } \\
\text { pneumonia, syphilis }\end{array}$ & Myocarditis & Pneumonia & - & - \\
\hline 138 & 651 & c. 41 years & $\mathbf{M}$ & Myocarditis & - & - & - & - \\
\hline
\end{tabular}




\begin{tabular}{|c|c|c|c|c|c|c|c|c|}
\hline \multirow{2}{*}{ No. } & \multirow{2}{*}{ Terry No. } & \multirow{2}{*}{$\begin{array}{c}\text { Age } \\
\text { at death }\end{array}$} & \multirow{2}{*}{ Sex } & \multicolumn{4}{|c|}{ Cause of death } & \multirow{2}{*}{$\begin{array}{l}\text { Included or not } \\
\text { into the evaluation }\end{array}$} \\
\hline & & & & MR & DC1 & DC2 & DC3 & \\
\hline 139 & $657 R$ & c. 28 years & $\mathbf{F}$ & Mitral stenosis & - & - & - & + \\
\hline 140 & 661 & c. 65 years & $\mathbf{F}$ & Uraemia & Uraemia & Nephritis & - & - \\
\hline 141 & 678R & c. 82 years & $\mathbf{F}$ & Heat stroke & - & - & - & - \\
\hline 142 & $685 R$ & c. 95 years & $\mathbf{F}$ & $\begin{array}{l}\text { Hypertensive } \\
\text { heart disease }\end{array}$ & $\begin{array}{l}\text { Hypertensive } \\
\text { heart disease }\end{array}$ & Cerebral thrombosis & - & - \\
\hline 143 & 686 & c. 78 years & $\mathbf{F}$ & $\begin{array}{c}\text { Myocarditis, } \\
\text { senile dementia }\end{array}$ & - & - & - & + \\
\hline 144 & 693 & 57 years & $\mathbf{M}$ & Pneumonia & - & - & - & - \\
\hline 145 & 694 & c. 52 years & $\mathbf{M}$ & $\begin{array}{l}\text { Myocarditis, } \\
\text { arteriosclerosis }\end{array}$ & Myocarditis & Arteriosclerosis & - & + \\
\hline 146 & 697 & 71 years & $\mathbf{M}$ & Myocarditis & Myocarditis & Nephritis & - & - \\
\hline 147 & $702 R$ & 47 years & M & Myocardial insufficiency & $\begin{array}{l}\text { Hypertensive } \\
\text { heart disease }\end{array}$ & Nephritis & - & + \\
\hline 148 & 704 & 40 years & $\mathbf{M}$ & Nephritis & - & - & - & - \\
\hline 149 & 712 & 47 years & $\mathbf{M}$ & Cardiorenal disease & Myocarditis & Nephritis & - & - \\
\hline 150 & 723 & 22 years & $\mathbf{F}$ & Lues & Syphilis & - & - & - \\
\hline 151 & 726 & c. 62 years & $\mathbf{M}$ & $\begin{array}{l}\text { Myocarditis, } \\
\text { syphilis }\end{array}$ & - & - & - & + \\
\hline 152 & 727 & c. 45 years & $\mathbf{M}$ & Gastric carcinoma & - & - & - & + \\
\hline
\end{tabular}




\begin{tabular}{|c|c|c|c|c|c|c|c|c|}
\hline \multirow{2}{*}{ No. } & \multirow{2}{*}{ Terry No. } & \multirow{2}{*}{$\begin{array}{c}\text { Age } \\
\text { at death }\end{array}$} & \multirow{2}{*}{ Sex } & \multicolumn{4}{|c|}{ Cause of death } & \multirow{2}{*}{$\begin{array}{l}\text { Included or not } \\
\text { into the evaluation }\end{array}$} \\
\hline & & & & MR & DC1 & DC2 & DC3 & \\
\hline 153 & 731 & c. 63 years & $\mathbf{M}$ & $\begin{array}{c}\text { Myocarditis, } \\
\text { rheumatic fever }\end{array}$ & - & - & - & - \\
\hline 154 & 745 & c. 69 years & $\mathbf{F}$ & $\begin{array}{c}\text { Heart failure, } \\
\text { bronchitis, syphilis }\end{array}$ & Myocarditis & Syphilis & - & - \\
\hline 155 & 747 & 45 years & $\mathbf{M}$ & Pyelitis & Peritonitis & Ulcerative sigmoiditis & - & - \\
\hline 156 & 748 & 85 years & $\mathbf{M}$ & Senile dementia & Myocarditis & Arteriosclerosis & - & - \\
\hline 157 & 759 & 60 years & $\mathbf{M}$ & Cardiorenal disease & Myocarditis & - & - & + \\
\hline 158 & 762 & 59 years & $\mathbf{M}$ & Syphilis & Myocarditis & Hypertrophied prostate & - & - \\
\hline 159 & 763 & 46 years & $\mathbf{M}$ & Pneumonia & - & - & - & - \\
\hline 160 & 766 & c. 35 years & $\mathbf{F}$ & - & - & - & - & - \\
\hline 161 & 781 & c. 60 years & $\mathbf{F}$ & Heart disease & - & - & - & - \\
\hline 162 & 782 & c. 30 years & $\mathbf{M}$ & Stab wound & Stabbed in back & - & - & - \\
\hline 163 & 789 & 40 years & $\mathbf{M}$ & Syphilis & - & - & - & + \\
\hline 164 & 795 & c. 57 years & $\mathbf{M}$ & $\begin{array}{c}\text { Carcinoma } \\
\text { of the cervical glands }\end{array}$ & - & - & - & + \\
\hline 165 & 806 & 78 years & $\mathbf{M}$ & $\begin{array}{l}\text { Myocarditis, } \\
\text { senility }\end{array}$ & - & - & - & - \\
\hline 166 & 809R & 60 years & $\mathbf{M}$ & $\begin{array}{l}\text { Hypertensive } \\
\text { heart disease }\end{array}$ & - & - & - & + \\
\hline
\end{tabular}




\begin{tabular}{|c|c|c|c|c|c|c|c|c|}
\hline \multirow{2}{*}{ No. } & \multirow{2}{*}{ Terry No. } & \multirow{2}{*}{$\begin{array}{c}\text { Age } \\
\text { at death }\end{array}$} & \multirow{2}{*}{ Sex } & \multicolumn{4}{|c|}{ Cause of death } & \multirow{2}{*}{$\begin{array}{l}\text { Included or not } \\
\text { into the evaluation }\end{array}$} \\
\hline & & & & MR & DC1 & DC2 & DC3 & \\
\hline 167 & 812 & c. 56 years & $\mathbf{M}$ & $\begin{array}{l}\text { Myocarditis, } \\
\text { arteriosclerosis }\end{array}$ & - & - & - & - \\
\hline 168 & 813 & 60 years & $\mathbf{M}$ & $\begin{array}{l}\text { Myocarditis, } \\
\text { arteriosclerosis, } \\
\text { gangrenous leg }\end{array}$ & - & - & - & - \\
\hline 169 & 815 & c. 32 years & $\mathbf{F}$ & $\begin{array}{c}\text { Homicide } \\
\text { by firearms }\end{array}$ & - & - & - & - \\
\hline 170 & 823 & c. 60 years & $\mathbf{M}$ & Cerebral apoplexy & - & - & - & + \\
\hline 171 & 833R & 47 years & $\mathbf{M}$ & Blastomycosis & - & - & - & + \\
\hline 172 & $834 R$ & 87 years & $\mathbf{F}$ & $\begin{array}{l}\text { Degenerative } \\
\text { heart disease }\end{array}$ & Arteriosclerosis & - & - & + \\
\hline 173 & 863 & 73 years & $\mathbf{M}$ & Senility & Cardiorenal disease & Pneumonia & - & + \\
\hline 174 & 868 & 60 years & $\mathbf{M}$ & Myocarditis & - & - & - & - \\
\hline 175 & 870 & 60 years & $\mathbf{M}$ & Parkinson's disease & Myocarditis & Parkinson's disease & - & - \\
\hline 176 & 872 & 48 years & $\mathbf{M}$ & Pneumonia & Lead poisoning & - & - & - \\
\hline 177 & 875 & c. 50 years & $\mathbf{M}$ & Intestinal obstruction & Erysipelas & - & - & - \\
\hline 178 & 878 & 72 years & $\mathbf{M}$ & Gastric carcinoma & - & - & - & - \\
\hline 179 & 879 & 50 years & M & Myocarditis & - & - & - & - \\
\hline 180 & 880 & c. 27 years & $\mathbf{F}$ & Nephritis & Myocarditis & Arthritis & - & - \\
\hline
\end{tabular}




\begin{tabular}{|c|c|c|c|c|c|c|c|c|}
\hline \multirow{2}{*}{ No. } & \multirow{2}{*}{ Terry No. } & \multirow{2}{*}{$\begin{array}{c}\text { Age } \\
\text { at death }\end{array}$} & \multirow{2}{*}{ Sex } & \multicolumn{4}{|c|}{ Cause of death } & \multirow{2}{*}{$\begin{array}{l}\text { Included or not } \\
\text { into the evaluation }\end{array}$} \\
\hline & & & & MR & DC1 & DC2 & DC3 & \\
\hline 181 & 881 & c. 24 years & $\mathbf{M}$ & Chancroid ulcers & Nephritis & - & - & - \\
\hline 182 & 891 & 39 years & $\mathbf{F}$ & Pneumonia & - & - & - & + \\
\hline 183 & 903R & c. 51 years & $\mathbf{F}$ & - & $\begin{array}{l}\text { Subdural brain } \\
\text { haemorrhage from fall }\end{array}$ & - & - & + \\
\hline 184 & 904R & 52 years & $\mathbf{F}$ & $\begin{array}{l}\text { Degenerative } \\
\text { heart disease }\end{array}$ & $\begin{array}{l}\text { Degenerative } \\
\text { heart disease }\end{array}$ & Arteriosclerosis & Diabetes mellitus & - \\
\hline 185 & 908 & c. 52 years & $\mathbf{M}$ & Lip cancer & - & - & - & - \\
\hline 186 & 919 & c. 55 years & $\mathbf{M}$ & Myocarditis & - & - & - & + \\
\hline 187 & 930R & c. 80 years & $\mathbf{F}$ & Cerebral apoplexy & - & - & - & + \\
\hline 188 & 934 & 62 years & $\mathbf{F}$ & $\begin{array}{l}\text { Myocarditis, } \\
\text { heart failure }\end{array}$ & - & - & - & + \\
\hline 189 & 938 & 73 years & M & $\begin{array}{l}\text { Myocarditis, } \\
\text { sigmoid cancer }\end{array}$ & Pneumonia & Myocarditis & - & + \\
\hline 190 & 941 & 59 years & $\mathbf{M}$ & Cardiac condition & Myocarditis & - & - & + \\
\hline 191 & 942 & 49 years & $\mathbf{M}$ & Rheumatism? & Pellagra & - & - & - \\
\hline 192 & 946 & 48 years & $\mathbf{M}$ & Pneumonia & - & - & - & + \\
\hline 193 & 948 & 39 years & $\mathbf{F}$ & Heart disease & Myocarditis & - & - & + \\
\hline 194 & 949 & 24 years & $\mathbf{F}$ & Nephritis & - & - & - & - \\
\hline
\end{tabular}




\begin{tabular}{|c|c|c|c|c|c|c|c|c|}
\hline \multirow{2}{*}{ No. } & \multirow{2}{*}{ Terry No. } & \multirow{2}{*}{$\begin{array}{c}\text { Age } \\
\text { at death }\end{array}$} & \multirow{2}{*}{ Sex } & \multicolumn{4}{|c|}{ Cause of death } & \multirow{2}{*}{$\begin{array}{l}\text { Included or not } \\
\text { into the evaluation }\end{array}$} \\
\hline & & & & MR & DC1 & DC2 & DC3 & \\
\hline 195 & 951R & c. 62 years & $\mathbf{F}$ & $\begin{array}{l}\text { Incarcerated } \\
\text { umbilical hernia }\end{array}$ & - & - & - & - \\
\hline 196 & 957R & 76 years & $\mathbf{F}$ & Femur fracture & - & - & - & + \\
\hline 197 & 963 & 71 years & $\mathbf{M}$ & Cardiac decompensation & Myocarditis & - & - & - \\
\hline 198 & 964 & 49 years & $\mathbf{M}$ & Cerebral apoplexy & Cerebral haemorrhage & - & - & + \\
\hline 199 & 968 & 48 years & $\mathbf{M}$ & Neurological condition & Lues & - & - & + \\
\hline 200 & 973 & 66 years & $\mathbf{M}$ & Pneumonia & Myocarditis & Pneumonia & - & + \\
\hline 201 & 974 & 77 years & $\mathbf{M}$ & Influenza & Myocarditis & Arteriosclerosis & - & - \\
\hline 202 & 979 & 85 years & $\mathbf{M}$ & $\begin{array}{c}\text { Myocarditis, } \\
\text { hypertension, senility }\end{array}$ & - & - & - & - \\
\hline 203 & 997 & c. 88 years & $\mathbf{F}$ & Myocarditis, senility & - & - & - & - \\
\hline 204 & 1023 & c. 20 years & $\mathbf{M}$ & Car accident & - & - & - & + \\
\hline 205 & $1029 R$ & 30 years & $\mathbf{M}$ & Parkinson's disease & - & - & - & + \\
\hline 206 & 1040 & 69 years & $\mathbf{M}$ & $\begin{array}{l}\text { Penile cancer } \\
\text { with metastasis }\end{array}$ & - & - & - & - \\
\hline 207 & 1045 & c. 41 years & $\mathbf{M}$ & Nephritis & Pulmonary embolism & Myocarditis & Nephritis & + \\
\hline 208 & 1046 & c. 60 years & $\mathbf{M}$ & - & $\begin{array}{l}\text { Hypertensive } \\
\text { heart disease }\end{array}$ & - & - & + \\
\hline
\end{tabular}




\begin{tabular}{|c|c|c|c|c|c|c|c|c|}
\hline \multirow{2}{*}{ No. } & \multirow{2}{*}{ Terry No. } & \multirow{2}{*}{$\begin{array}{c}\text { Age } \\
\text { at death }\end{array}$} & \multirow{2}{*}{ Sex } & \multicolumn{4}{|c|}{ Cause of death } & \multirow{2}{*}{$\begin{array}{l}\text { Included or not } \\
\text { into the evaluation }\end{array}$} \\
\hline & & & & MR & DC1 & DC2 & DC3 & \\
\hline 209 & 1050 & 64 years & $\mathbf{M}$ & Intestinal stasis & Intestinal obstruction & Peritonitis & - & + \\
\hline 210 & 1051 & 74 years & $\mathbf{M}$ & $\begin{array}{l}\text { Gastrointestinal } \\
\text { carcinoma }\end{array}$ & Gastroenteritis & - & - & - \\
\hline 211 & 1058 & 68 years & $\mathbf{F}$ & Myocarditis & Cerebral apoplexy & Cerebral haemorrhage & Myocarditis & + \\
\hline 212 & 1060 & c. 65 years & $\mathbf{M}$ & $\begin{array}{l}\text { Asthma, } \\
\text { myocarditis }\end{array}$ & - & - & - & + \\
\hline 213 & 1066R & 62 years & $\mathbf{M}$ & Pneumonia & $\begin{array}{l}\text { Degenerative } \\
\text { heart disease }\end{array}$ & Arteriosclerosis & - & + \\
\hline 214 & 1070 & c. 51 years & $\mathbf{M}$ & Nephritis & Myocarditis & Nephritis & - & + \\
\hline 215 & 1071R & c. 70 years & $\mathbf{F}$ & Accident & Endocarditis & $\begin{array}{c}\text { Stenosis, } \\
\text { hip fracture }\end{array}$ & - & + \\
\hline 216 & 1081 & 50 years & $\mathbf{M}$ & Cardiorenal disease & Myocarditis & - & - & - \\
\hline 217 & 1098 & c. 60 years & $\mathbf{M}$ & Cerebral haemorrhage & Cerebral haemorrhage & Arteriosclerosis & - & + \\
\hline 218 & 1100RR & c. 76 years & $\mathbf{F}$ & Brain syndrome & $\begin{array}{l}\text { Arteriosclerotic } \\
\text { heart disease }\end{array}$ & Brain syndrome & - & + \\
\hline 219 & $1102 R$ & c. 76 years & $\mathbf{F}$ & Coronary thrombosis & Coronary thrombosis & $\begin{array}{l}\text { Arteriosclerotic } \\
\text { heart disease }\end{array}$ & - & + \\
\hline 220 & 1107 & 78 years & $\mathbf{M}$ & $\begin{array}{l}\text { Myocarditis, } \\
\text { senility }\end{array}$ & - & - & - & + \\
\hline 221 & 1116 & 80 years & $\mathbf{M}$ & - & Myocarditis & Nephritis & Hypertrophied prostate & - \\
\hline 222 & 1130R & 79 years & $\mathbf{M}$ & Convulsions & Cerebral haemorrhage & Arteriosclerosis & - & + \\
\hline
\end{tabular}




\begin{tabular}{|c|c|c|c|c|c|c|c|c|}
\hline \multirow{2}{*}{ No. } & \multirow{2}{*}{ Terry No. } & \multirow{2}{*}{$\begin{array}{c}\text { Age } \\
\text { at death }\end{array}$} & \multirow{2}{*}{ Sex } & \multicolumn{4}{|c|}{ Cause of death } & \multirow{2}{*}{$\begin{array}{l}\text { Included or not } \\
\text { into the evaluation }\end{array}$} \\
\hline & & & & MR & DC1 & DC2 & DC3 & \\
\hline 223 & 1133RR & c. 80 years & $\mathbf{F}$ & $\begin{array}{l}\text { Arteriosclerosis, } \\
\text { avitaminosis, } \\
\text { dehydration }\end{array}$ & Malnutrition & Senility & - & + \\
\hline 224 & $1134 R$ & 70 years & $\mathbf{F}$ & Hyperarthritis & Arteriosclerosis & Senile psychosis & Hyperarthritis & + \\
\hline 225 & 1135 & c. 62 years & $\mathbf{F}$ & Pneumonia & - & - & - & - \\
\hline 226 & $1137 R$ & c. 78 years & $\mathbf{F}$ & Pneumonia & $\begin{array}{l}\text { Degenerative } \\
\text { heart disease }\end{array}$ & Pneumonia & Arteriosclerosis & + \\
\hline 227 & 1138R & 37 years & $\mathbf{M}$ & Pneumonia & Pneumonia & Pleural effusion & - & + \\
\hline 228 & 1140 & 46 years & $\mathbf{M}$ & Cardiac decompensation & Myocarditis & - & - & + \\
\hline 229 & 1161RR & c. 56 years & $\mathbf{F}$ & Hernia & Intestinal obstruction & Incisional hernia & - & - \\
\hline 230 & 1163 & 41 years & $\mathbf{F}$ & Pellagra & - & - & - & + \\
\hline 231 & 1182R & c. 48 years & $\mathbf{F}$ & Pneumonia & Aortitis & - & - & + \\
\hline 232 & 1186 & c. 44 years & $\mathbf{F}$ & Lung abscess & Lung abscess & Pneumonia & - & + \\
\hline 233 & 1192 & 58 years & $\mathbf{M}$ & Pneumonia & - & - & - & + \\
\hline 234 & 1198 & c. 42 years & $\mathbf{F}$ & Hypertension & Myocarditis & - & - & - \\
\hline 235 & $1204 R$ & 74 years & $\mathbf{M}$ & Heart disease & $\begin{array}{l}\text { Arteriosclerotic } \\
\text { heart disease }\end{array}$ & - & - & + \\
\hline 236 & 1219 & 79 years & $\mathbf{M}$ & $\begin{array}{l}\text { Myocarditis, } \\
\text { nephritis }\end{array}$ & Myocarditis & Senility & - & + \\
\hline
\end{tabular}




\begin{tabular}{|c|c|c|c|c|c|c|c|c|}
\hline \multirow{2}{*}{ No. } & \multirow{2}{*}{ Terry No. } & \multirow{2}{*}{$\begin{array}{c}\text { Age } \\
\text { at death }\end{array}$} & \multirow{2}{*}{ Sex } & \multicolumn{4}{|c|}{ Cause of death } & \multirow{2}{*}{$\begin{array}{l}\text { Included or not } \\
\text { into the evaluation }\end{array}$} \\
\hline & & & & MR & DC1 & DC2 & DC3 & \\
\hline 237 & 1224 & 60 years & $\mathbf{M}$ & Mediastinal tumour & Aortic aneurysm & - & - & + \\
\hline 238 & 1228 & 79 years & $\mathbf{M}$ & $\begin{array}{l}\text { Nephritis, } \\
\text { pneumonia }\end{array}$ & Myocarditis & Pneumonia & - & + \\
\hline 239 & 1229 & c. 73 years & $\mathbf{M}$ & Gangrenous foot & Gangrenous foot & Arteriosclerosis & Myocarditis & + \\
\hline 240 & 1232 & 57 years & $\mathbf{M}$ & $\begin{array}{c}\text { Pneumonia, myocarditis, } \\
\text { arteriosclerosis }\end{array}$ & - & - & - & + \\
\hline 241 & $1243 R$ & c. 49 years & $\mathbf{F}$ & Myocarditis & - & - & - & + \\
\hline 242 & 1252 & 50 years & $\mathbf{F}$ & Uterine fibroid & Nephritis & Uraemia & - & + \\
\hline 243 & 1267 & c. 78 years & $\mathbf{M}$ & $\begin{array}{l}\text { Myocarditis, } \\
\text { senility }\end{array}$ & - & - & - & + \\
\hline 244 & 1271 & 58 years & $\mathbf{M}$ & Lues & Lues & Myocarditic & $\begin{array}{c}\text { Cardiac } \\
\text { decompensation }\end{array}$ & + \\
\hline 245 & 1277 & c. 59 years & $\mathbf{M}$ & Heat stroke & Epilepsy & - & - & + \\
\hline 246 & 1281 & c. 53 years & $\mathbf{M}$ & Hepatic carcinoma & Hepatic carcinoma & Ascites & - & - \\
\hline 247 & 1288R & c. 58 years & $\mathbf{F}$ & Nephritis & Nephritis & Arteriosclerosis & - & - \\
\hline 248 & 1290 & 59 years & $\mathbf{F}$ & Myocarditis & Myocarditis & Nephritis & - & - \\
\hline 249 & 1291 & c. 52 years & $\mathbf{M}$ & Cardiac decompensation & $\begin{array}{l}\text { Hypertensive } \\
\text { heart disease }\end{array}$ & - & - & + \\
\hline 250 & 1299R & 52 years & $\mathbf{M}$ & Gastric ulcer & Gastric ulcer & Lues & - & + \\
\hline
\end{tabular}




\begin{tabular}{|c|c|c|c|c|c|c|c|c|}
\hline \multirow{2}{*}{ No. } & \multirow{2}{*}{ Terry No. } & \multirow{2}{*}{$\begin{array}{c}\text { Age } \\
\text { at death }\end{array}$} & \multirow{2}{*}{ Sex } & \multicolumn{4}{|c|}{ Cause of death } & \multirow{2}{*}{$\begin{array}{l}\text { Included or not } \\
\text { into the evaluation }\end{array}$} \\
\hline & & & & MR & DC1 & DC2 & DC3 & \\
\hline 251 & 1310 & c. 85 years & $\mathbf{M}$ & Cardiovascular reactivity & $\begin{array}{l}\text { Arteriosclerotic } \\
\text { heart disease }\end{array}$ & Auricular fibrillation & - & + \\
\hline 252 & 1314 & c. 61 years & $\mathbf{F}$ & $\begin{array}{c}\text { Myocarditis, } \\
\text { nephritis, senility }\end{array}$ & - & - & - & - \\
\hline 253 & 1323 & 50 years & $\mathbf{M}$ & Myocarditis & - & - & - & - \\
\hline 254 & 1336 & 68 years & $\mathbf{M}$ & Pneumonia & - & - & - & - \\
\hline 255 & 1342 & c. 74 years & $\mathbf{M}$ & $\begin{array}{l}\text { Hypertensive } \\
\text { heart disease }\end{array}$ & - & - & - & + \\
\hline 256 & 1343 & c. 73 years & $\mathbf{F}$ & $\begin{array}{c}\text { Heart failure, } \\
\text { nephritis }\end{array}$ & Myocarditis & Nephritis & Hypertension & + \\
\hline 257 & 1347R & 38 years & $\mathbf{F}$ & Cardiorenal disease & $\begin{array}{l}\text { Hypertensive } \\
\text { heart disease }\end{array}$ & - & - & + \\
\hline 258 & 1349 & 86 years & $\mathbf{M}$ & $\begin{array}{l}\text { Cerebral accident, } \\
\text { haemorrhage }\end{array}$ & Cerebral haemorrhage & Senility & $\begin{array}{l}\text { Old cerebral } \\
\text { haemorrhage }\end{array}$ & - \\
\hline 259 & 1353R & c. 50 years & $\mathbf{F}$ & Pneumonia & - & - & - & + \\
\hline 260 & 1355R & 73 years & $\mathbf{F}$ & Asthma & $\begin{array}{l}\text { Arteriosclerotic } \\
\text { heart disease }\end{array}$ & - & - & - \\
\hline 261 & 1368 & 37 years & $\mathbf{M}$ & Appendicitis & Gangrenous appendix & Pneumonia & - & + \\
\hline 262 & 1375R & c. 38 years & $\mathbf{M}$ & Pneumonia & - & - & - & + \\
\hline 263 & 1376 & 46 years & $\mathbf{M}$ & Pneumonia & Pneumonia & Myocarditis & - & + \\
\hline 264 & 1378 & 54 years & $\mathbf{M}$ & Peritonitis & - & - & - & + \\
\hline
\end{tabular}




\begin{tabular}{|c|c|c|c|c|c|c|c|c|}
\hline \multirow{2}{*}{ No. } & \multirow{2}{*}{ Terry No. } & \multirow{2}{*}{$\begin{array}{c}\text { Age } \\
\text { at death }\end{array}$} & \multirow{2}{*}{ Sex } & \multicolumn{4}{|c|}{ Cause of death } & \multirow{2}{*}{$\begin{array}{l}\text { Included or not } \\
\text { into the evaluation }\end{array}$} \\
\hline & & & & MR & DC1 & DC2 & DC3 & \\
\hline 265 & 1387 & 40 years & $\mathbf{M}$ & Lues & - & - & - & + \\
\hline 266 & 1391R & c. 65 years & $\mathbf{F}$ & Myocarditis & - & - & - & - \\
\hline 267 & 1399 & c. 37 years & $\mathbf{M}$ & Brain abscess & - & - & - & - \\
\hline 268 & 1405 & 75 years & $\mathbf{F}$ & Myocarditis & - & - & - & + \\
\hline 269 & 1411R & 65 years & $\mathbf{M}$ & - & $\begin{array}{l}\text { Nephritis, } \\
\text { uraemia }\end{array}$ & Hypertrophied prostate & - & + \\
\hline 270 & 1416 & c. 70 years & $\mathbf{F}$ & $\begin{array}{l}\text { Hypertensive } \\
\text { heart disease }\end{array}$ & $\begin{array}{l}\text { Arteriosclerotic } \\
\text { heart disease }\end{array}$ & Senile psychosis & - & + \\
\hline 271 & 1417R & 37 years & $\mathbf{F}$ & Dysentery & $\begin{array}{l}\text { Perianal \& ischiorectal } \\
\text { abscesses }\end{array}$ & Staphylococcosis & Secondary anaemia & + \\
\hline 272 & 1435 & 66 years & $\mathbf{F}$ & $\begin{array}{l}\text { Myocarditis, } \\
\text { arteriosclerosis }\end{array}$ & - & - & - & + \\
\hline 273 & 1439R & 56 years & $\mathbf{M}$ & Epilepsy, hypertension & Pneumonia & Arteriosclerotic dementia & - & + \\
\hline 274 & 1444 & 59 years & $\mathbf{M}$ & Heart disease & $\begin{array}{l}\text { Arteriosclerotic } \\
\text { heart disease }\end{array}$ & - & - & + \\
\hline 275 & 1460R & 58 years & $\mathbf{M}$ & Hypertrophied prostate & Hypertrophied prostate & Nephritis & - & - \\
\hline 276 & 1462 & c. 54 years & $\mathbf{F}$ & Myocarditis & Myocarditis & Syphilis & - & - \\
\hline 277 & 1467 & 73 years & $\mathbf{M}$ & Gastric malignancy & $\begin{array}{l}\text { Arteriosclerotic } \\
\text { heart disease }\end{array}$ & Cardiac decompensation & - & + \\
\hline 278 & 1492 & 65 years & $\mathbf{M}$ & $\begin{array}{l}\text { Myocarditis, } \\
\text { pneumonia }\end{array}$ & - & - & - & - \\
\hline
\end{tabular}




\begin{tabular}{|c|c|c|c|c|c|c|c|c|}
\hline \multirow{2}{*}{ No. } & \multirow{2}{*}{ Terry No. } & \multirow{2}{*}{$\begin{array}{c}\text { Age } \\
\text { at death }\end{array}$} & \multirow{2}{*}{ Sex } & \multicolumn{4}{|c|}{ Cause of death } & \multirow{2}{*}{$\begin{array}{l}\text { Included or not } \\
\text { into the evaluation }\end{array}$} \\
\hline & & & & MR & DC1 & DC2 & DC3 & \\
\hline 279 & 1495 & 89 years & $\mathbf{M}$ & Myocarditis & Myocarditis & Pneumonia & - & + \\
\hline 280 & $1502 R$ & c. 82 years & $\mathbf{F}$ & Arteriosclerosis & Arteriosclerosis & Senility & - & + \\
\hline 281 & $1505 R$ & 41 years & $\mathbf{F}$ & Hemiplegia & Hypertension & Cerebral haemorrhage & - & + \\
\hline 282 & 1514 & c. 70 years & $\mathbf{M}$ & $\begin{array}{l}\text { Hemiplegia, } \\
\text { strictures }\end{array}$ & - & - & - & + \\
\hline 283 & 1515 & 43 years & $\mathbf{F}$ & $\begin{array}{l}\text { Hypertensive } \\
\text { heart disease }\end{array}$ & Cerebral haemorrhage & $\begin{array}{l}\text { Hypertensive } \\
\text { heart disease }\end{array}$ & - & - \\
\hline 284 & 1519 & 71 years & $\mathbf{M}$ & Myocarditis & $\begin{array}{l}\text { Myocarditis, cardiac } \\
\text { decompensation }\end{array}$ & - & - & + \\
\hline 285 & 1534 & 44 years & $\mathbf{M}$ & Urinary bladder calculi & $\begin{array}{l}\text { Pyelonephritis, } \\
\text { catarrhal cystitis }\end{array}$ & $\begin{array}{l}\text { Bladder \& urethral } \\
\text { calculi }\end{array}$ & - & + \\
\hline 286 & 1541R & c. 57 years & $\mathbf{F}$ & $?$ & Coronary occlusion & $\begin{array}{c}\text { Arteriosclerotic } \\
\text { heart disease }\end{array}$ & - & - \\
\hline 287 & 1543 & c. 70 years & $\mathbf{M}$ & Thrombosis & - & - & - & + \\
\hline 288 & 1549 & c. 40 years & $\mathbf{F}$ & Cerebral apoplexy & - & - & - & + \\
\hline 289 & 1552 & 84 years & $\mathbf{F}$ & $\begin{array}{l}\text { Hypertension, } \\
\text { senility }\end{array}$ & $\begin{array}{c}\text { Cardiac } \\
\text { decompensation }\end{array}$ & $\begin{array}{l}\text { Arteriosclerotic } \\
\text { vascular disease }\end{array}$ & - & + \\
\hline 290 & 1554 & c. 56 years & $\mathbf{F}$ & $\begin{array}{l}\text { Hypertensive } \\
\text { heart disease }\end{array}$ & - & - & - & + \\
\hline 291 & 1567 & c. 69 years & $\mathbf{F}$ & $\begin{array}{c}\text { Stroke, } \\
\text { heart failure }\end{array}$ & - & - & - & + \\
\hline 292 & 1581 & 64 years & $\mathbf{F}$ & $\begin{array}{l}\text { Arteriosclerotic heart } \\
\text { disease }\end{array}$ & - & - & - & + \\
\hline
\end{tabular}




\begin{tabular}{|c|c|c|c|c|c|c|c|c|}
\hline \multirow{2}{*}{ No. } & \multirow{2}{*}{ Terry No. } & \multirow{2}{*}{$\begin{array}{c}\text { Age } \\
\text { at death }\end{array}$} & \multirow{2}{*}{ Sex } & \multicolumn{4}{|c|}{ Cause of death } & \multirow{2}{*}{$\begin{array}{l}\text { Included or not } \\
\text { into the evaluation }\end{array}$} \\
\hline & & & & MR & DC1 & DC2 & DC3 & \\
\hline 293 & 1587 & c. 76 years & $\mathbf{F}$ & Coronary vessel disease & Coronary vessel disease & Pneumonia & - & - \\
\hline 294 & 1592 & 53 years & $\mathbf{F}$ & $\begin{array}{l}\text { Arteriosclerotic } \\
\text { heart disease }\end{array}$ & - & - & - & + \\
\hline 295 & 1599 & c. 41 years & $\mathbf{F}$ & Suicide & - & - & - & + \\
\hline 296 & 1600 & 26 years & $\mathbf{F}$ & Alcoholism, epilepsy & Pneumonia & - & - & - \\
\hline 297 & 1604 & 50 years & $\mathbf{F}$ & Cerebral haemorrhage & Cerebral haemorrhage & $\begin{array}{l}\text { Hypertensive } \\
\text { vascular disease }\end{array}$ & - & + \\
\hline 298 & 1614 & 38 years & $\mathbf{F}$ & - & $\begin{array}{c}\text { Congestive } \\
\text { heart failure }\end{array}$ & $\begin{array}{c}\text { Rheumatic } \\
\text { mitral valve disease }\end{array}$ & Mental deficiency & + \\
\hline 299 & 1627 & c. 90 years & $\mathbf{F}$ & $\begin{array}{l}\text { Hypertensive heart } \\
\text { disease, failure }\end{array}$ & $\begin{array}{l}\text { Arteriosclerotic } \\
\text { heart disease }\end{array}$ & - & - & + \\
\hline 300 & 1631 & 83 years & $\mathbf{F}$ & $\begin{array}{l}\text { Arteriosclerotic } \\
\text { heart disease }\end{array}$ & - & - & - & - \\
\hline 301 & 1633 & 80 years & $\mathbf{F}$ & Coronary occlusion & Coronary occlusion & Coronary stenosis & - & - \\
\hline 302 & 1634 & 63 years & $\mathbf{F}$ & Coronary thrombosis & Coronary thrombosis & Arteriosclerosis & - & - \\
\hline
\end{tabular}


Supplementary table 3: Individual data regarding TBM-related endocranial alterations in the TB group (+= present; $-=$ not present).

\begin{tabular}{|c|c|c|c|c|c|}
\hline No. & Terry No. & APDIs & ABVIs & PAs & GIs \\
\hline 1 & 13R & + & + & - & - \\
\hline 2 & $23 R$ & - & + & - & - \\
\hline 3 & 30R & + & - & - & + \\
\hline 4 & $35 \mathrm{R}$ & - & - & - & - \\
\hline 5 & 39 & - & - & - & - \\
\hline 6 & 54 & + & - & - & - \\
\hline 7 & 84 & + & + & - & + \\
\hline 8 & $87 R$ & - & - & - & + \\
\hline 9 & 89R & + & - & - & - \\
\hline 10 & 90 & + & - & - & - \\
\hline 11 & 91R & - & - & - & - \\
\hline 12 & 95 & + & + & - & + \\
\hline 13 & 95R & + & - & - & - \\
\hline 14 & 103R & - & - & - & - \\
\hline 15 & 114 & - & - & - & - \\
\hline 16 & 128 & + & + & + & - \\
\hline 17 & 129 & + & - & - & + \\
\hline 18 & 130 & - & - & - & - \\
\hline 19 & 138 & + & - & - & + \\
\hline 20 & 139 & + & - & - & + \\
\hline 21 & 145R & - & + & - & + \\
\hline 22 & 146R & + & - & - & - \\
\hline 23 & 158R & - & + & - & + \\
\hline 24 & 182 & + & - & - & + \\
\hline 25 & 194 & + & - & - & - \\
\hline 26 & 204 & + & + & + & - \\
\hline 27 & 205 & + & - & - & + \\
\hline 28 & 207 & + & - & - & - \\
\hline 29 & 220 & + & + & - & + \\
\hline 30 & 222 & + & - & - & + \\
\hline 31 & 230 & + & - & - & + \\
\hline 32 & 232R & - & - & - & + \\
\hline 33 & 235 & + & + & - & - \\
\hline 34 & 248R & - & - & - & - \\
\hline 35 & 250 & - & - & - & - \\
\hline 36 & 251 & + & - & - & - \\
\hline 37 & 254 & + & + & + & - \\
\hline 38 & 255 & + & + & - & + \\
\hline
\end{tabular}




\begin{tabular}{|c|c|c|c|c|c|}
\hline No. & Terry No. & APDIs & ABVIs & PAs & GIs \\
\hline 39 & 264 & - & - & - & - \\
\hline 40 & 265 & + & + & - & - \\
\hline 41 & 267 & + & - & - & - \\
\hline 42 & 269 & + & - & - & + \\
\hline 43 & 270 & + & - & - & + \\
\hline 44 & 279 & + & + & - & + \\
\hline 45 & 280 & + & + & + & + \\
\hline 46 & 282 & - & - & - & - \\
\hline 47 & $283 R$ & + & - & - & - \\
\hline 48 & 284 & - & - & - & - \\
\hline 49 & 304 & + & + & + & - \\
\hline 50 & 306 & + & + & + & - \\
\hline 51 & 309 & - & - & - & - \\
\hline 52 & 318 & - & - & - & - \\
\hline 53 & 328R & - & - & - & - \\
\hline 54 & 329 & - & + & - & + \\
\hline 55 & 341 & + & - & - & + \\
\hline 56 & 353 & - & + & - & - \\
\hline 57 & 358R & - & + & - & - \\
\hline 58 & 382R & + & - & - & - \\
\hline 59 & 385 & + & - & + & - \\
\hline 60 & 386R & - & - & - & - \\
\hline 61 & 400 & + & - & - & - \\
\hline 62 & 402 & - & - & - & - \\
\hline 63 & 410R & - & - & - & - \\
\hline 64 & 423 & + & - & + & - \\
\hline 65 & 424 & - & - & - & - \\
\hline 66 & $426 R$ & - & - & - & - \\
\hline 67 & 432 & - & - & - & + \\
\hline 68 & 444 & - & - & - & + \\
\hline 69 & 466 & + & - & - & - \\
\hline 70 & 468 & + & - & - & - \\
\hline 71 & 490 & - & - & - & - \\
\hline 72 & 504 & - & - & - & + \\
\hline 73 & 522 & + & + & + & + \\
\hline 74 & 523 & - & - & - & - \\
\hline 75 & 537 & - & - & - & - \\
\hline 76 & 541 & - & - & - & + \\
\hline 77 & 549 & - & - & - & - \\
\hline 78 & 555 & - & - & - & - \\
\hline
\end{tabular}




\begin{tabular}{|c|c|c|c|c|c|}
\hline No. & Terry No. & APDIs & ABVIs & PAs & GIs \\
\hline 79 & 562 & + & - & - & + \\
\hline 80 & 565 & + & - & - & - \\
\hline 81 & 566 & - & - & - & + \\
\hline 82 & 568 & - & + & + & + \\
\hline 83 & 571 & - & + & - & - \\
\hline 84 & 572 & + & - & - & - \\
\hline 85 & 575 & - & - & - & + \\
\hline 86 & 583 & + & - & - & - \\
\hline 87 & 585 & + & - & - & - \\
\hline 88 & 592 & + & - & - & - \\
\hline 89 & 595 & + & - & - & + \\
\hline 90 & 620 & - & - & - & + \\
\hline 91 & 621R & + & + & + & - \\
\hline 92 & 626R & - & - & - & - \\
\hline 93 & 664 & + & - & - & + \\
\hline 94 & 669R & - & - & - & - \\
\hline 95 & 679 & - & - & - & - \\
\hline 96 & 680 & - & - & - & + \\
\hline 97 & $728 R$ & + & - & - & - \\
\hline 98 & 739 & - & + & - & + \\
\hline 99 & 752 & + & - & - & + \\
\hline 100 & 757 & + & - & - & - \\
\hline 101 & 761 & - & - & - & - \\
\hline 102 & 771 & + & - & - & - \\
\hline 103 & 776 & + & - & - & - \\
\hline 104 & 786 & + & - & - & - \\
\hline 105 & 799 & + & - & - & - \\
\hline 106 & 820R & + & - & - & - \\
\hline 107 & 822 & + & + & - & + \\
\hline 108 & 828 & + & - & - & + \\
\hline 109 & 844 & + & - & - & - \\
\hline 110 & 846 & + & - & + & - \\
\hline 111 & 876 & + & - & - & + \\
\hline 112 & 892 & + & - & - & - \\
\hline 113 & 895 & - & - & - & - \\
\hline 114 & 896RR & + & + & - & + \\
\hline 115 & 897 & + & - & + & - \\
\hline 116 & 902 & - & + & - & - \\
\hline 117 & 907 & + & - & - & - \\
\hline 118 & 914 & + & - & - & + \\
\hline
\end{tabular}




\begin{tabular}{|c|c|c|c|c|c|}
\hline No. & Terry No. & APDIs & ABVIs & PAs & GIs \\
\hline 119 & 915 & + & - & + & - \\
\hline 120 & 932 & + & + & + & - \\
\hline 121 & 933R & + & + & - & + \\
\hline 122 & 936 & + & - & - & - \\
\hline 123 & 950 & + & - & - & - \\
\hline 124 & 952 & - & - & - & + \\
\hline 125 & 955 & + & + & + & - \\
\hline 126 & 957 & - & - & - & - \\
\hline 127 & 975 & - & - & - & + \\
\hline 128 & 987 & + & + & + & + \\
\hline 129 & 1002 & + & - & - & - \\
\hline 130 & 1005 & + & - & - & - \\
\hline 131 & 1013 & + & - & - & - \\
\hline 132 & 1018 & + & - & - & + \\
\hline 133 & 1020 & + & + & - & + \\
\hline 134 & 1027 & - & + & + & + \\
\hline 135 & 1030 & - & + & - & - \\
\hline 136 & 1031 & + & - & - & - \\
\hline 137 & 1033 & + & + & + & - \\
\hline 138 & 1034 & + & - & + & - \\
\hline 139 & 1036 & + & - & - & - \\
\hline 140 & 1043 & - & - & - & - \\
\hline 141 & 1047 & + & - & - & - \\
\hline 142 & 1048 & + & - & - & - \\
\hline 143 & 1057 & + & - & + & + \\
\hline 144 & 1072 & + & - & - & - \\
\hline 145 & 1076 & + & + & - & + \\
\hline 146 & 1086 & - & - & - & - \\
\hline 147 & 1093 & + & - & - & - \\
\hline 148 & 1095 & + & - & - & - \\
\hline 149 & 1096R & + & - & - & + \\
\hline 150 & 1105 & + & + & + & - \\
\hline 151 & 1106 & + & - & - & + \\
\hline 152 & 1113 & + & - & + & - \\
\hline 153 & 1122 & + & + & + & - \\
\hline 154 & 1124R & - & - & - & - \\
\hline 155 & 1129 & + & - & - & - \\
\hline 156 & 1132 & + & - & - & - \\
\hline 157 & 1147R & - & - & - & - \\
\hline 158 & 1156 & + & - & - & - \\
\hline
\end{tabular}




\begin{tabular}{|c|c|c|c|c|c|}
\hline No. & Terry No. & APDIs & ABVIs & PAs & GIs \\
\hline 159 & 1157 & + & - & - & - \\
\hline 160 & 1159 & + & - & + & + \\
\hline 161 & 1165 & + & + & + & - \\
\hline 162 & 1169 & + & + & + & - \\
\hline 163 & 1173 & + & - & - & - \\
\hline 164 & 1183 & + & - & - & - \\
\hline 165 & 1185 & + & - & - & - \\
\hline 166 & 1187 & + & - & - & - \\
\hline 167 & 1190 & + & - & + & - \\
\hline 168 & 1205 & + & - & - & - \\
\hline 169 & 1210 & + & - & - & - \\
\hline 170 & 1215 & + & - & - & - \\
\hline 171 & 1222 & + & + & + & + \\
\hline 172 & 1226 & - & - & - & - \\
\hline 173 & 1230 & + & - & - & - \\
\hline 174 & 1236 & + & - & + & - \\
\hline 175 & 1247 & - & - & - & - \\
\hline 176 & 1249R & + & - & - & + \\
\hline 177 & 1255 & + & - & + & - \\
\hline 178 & 1263R & + & - & - & + \\
\hline 179 & 1264 & + & - & - & + \\
\hline 180 & 1266R & - & - & - & - \\
\hline 181 & 1275 & + & - & - & - \\
\hline 182 & 1278 & + & - & - & - \\
\hline 183 & 1282 & - & - & - & - \\
\hline 184 & 1285 & + & - & + & - \\
\hline 185 & 1287 & + & - & + & - \\
\hline 186 & 1300 & + & - & + & - \\
\hline 187 & 1304R & + & - & - & + \\
\hline 188 & 1309 & + & - & - & + \\
\hline 189 & 1313 & + & - & + & - \\
\hline 190 & 1315 & + & - & - & - \\
\hline 191 & 1318 & + & - & + & + \\
\hline 192 & 1319 & + & - & + & + \\
\hline 193 & 1322 & + & + & + & - \\
\hline 194 & 1331 & - & - & - & + \\
\hline 195 & 1337RR & - & - & - & - \\
\hline 196 & 1346 & - & - & - & - \\
\hline 197 & 1352 & - & - & - & - \\
\hline 198 & 1359 & + & - & + & - \\
\hline
\end{tabular}




\begin{tabular}{|c|c|c|c|c|c|}
\hline No. & Terry No. & APDIs & ABVIs & PAs & GIs \\
\hline 199 & 1362 & + & - & - & - \\
\hline 200 & 1367 & + & - & - & - \\
\hline 201 & 1369 & + & + & + & - \\
\hline 202 & 1377 & - & + & + & - \\
\hline 203 & 1379 & - & - & - & - \\
\hline 204 & 1388 & + & - & + & + \\
\hline 205 & 1397 & + & - & - & - \\
\hline 206 & 1398 & + & - & + & - \\
\hline 207 & 1401 & - & - & - & - \\
\hline 208 & 1406 & - & - & - & - \\
\hline 209 & 1407 & + & - & - & - \\
\hline 210 & 1419 & - & - & - & - \\
\hline 211 & 1422R & + & - & - & - \\
\hline 212 & 1428R & + & - & - & - \\
\hline 213 & 1434R & + & - & - & - \\
\hline 214 & 1451 & - & - & - & - \\
\hline 215 & 1453R & - & - & - & - \\
\hline 216 & 1455 & + & - & - & - \\
\hline 217 & 1458 & + & - & + & + \\
\hline 218 & 1476 & - & - & - & - \\
\hline 219 & 1503 & + & - & - & + \\
\hline 220 & 1507 & + & - & - & - \\
\hline 221 & 1521 & + & + & - & - \\
\hline 222 & 1531 & - & - & - & - \\
\hline 223 & 1533 & + & - & - & + \\
\hline 224 & 1536 & - & - & - & - \\
\hline 225 & 1539 & + & - & - & - \\
\hline 226 & 1544 & - & + & + & - \\
\hline 227 & 1551 & + & + & - & - \\
\hline 228 & 1553 & + & - & + & - \\
\hline 229 & 1555 & + & + & - & - \\
\hline 230 & 1562 & + & + & + & - \\
\hline 231 & 1568 & - & - & - & - \\
\hline 232 & 1572 & + & - & - & + \\
\hline 233 & 1576 & - & - & - & - \\
\hline 234 & 1629 & - & - & - & - \\
\hline
\end{tabular}


Supplementary table 4: Individual data regarding TBM-related endocranial alterations in the NTB group (+= present; $-=$ not present).

\begin{tabular}{|c|c|c|c|c|c|}
\hline No. & Terry No. & APDIs & ABVIs & PAs & GIs \\
\hline 1 & $4 R$ & - & - & - & + \\
\hline 2 & $12 R$ & - & + & + & - \\
\hline 3 & 19R & + & - & - & - \\
\hline 4 & 25 & - & - & - & - \\
\hline 5 & $25 R$ & - & + & - & - \\
\hline 6 & $31 R$ & - & - & - & - \\
\hline 7 & $44 R$ & - & - & - & - \\
\hline 8 & $46 R$ & - & - & - & - \\
\hline 9 & $47 R$ & - & - & - & - \\
\hline 10 & $58 \mathrm{R}$ & + & - & + & - \\
\hline 11 & 62RR & + & - & - & - \\
\hline 12 & $64 R$ & + & - & - & - \\
\hline 13 & 69 & - & - & - & - \\
\hline 14 & $76 R$ & - & - & - & - \\
\hline 15 & $79 R$ & - & - & - & - \\
\hline 16 & 104RR & - & - & - & - \\
\hline 17 & 105R & - & - & - & - \\
\hline 18 & 112R & + & - & - & - \\
\hline 19 & $124 R$ & - & - & - & - \\
\hline 20 & 127R & + & + & - & - \\
\hline 21 & 132R & - & - & - & - \\
\hline 22 & 134 & - & - & - & - \\
\hline 23 & 135R & + & - & - & - \\
\hline 24 & 140RR & - & + & - & - \\
\hline 25 & 141R & - & - & - & - \\
\hline 26 & 142R & - & - & - & - \\
\hline 27 & 149R & - & - & - & - \\
\hline 28 & 167 & + & - & - & - \\
\hline 29 & 177R & - & - & - & - \\
\hline 30 & 178R & - & - & + & - \\
\hline 31 & 179R & - & - & - & - \\
\hline 32 & 197R & + & - & - & + \\
\hline 33 & 199 & - & - & - & - \\
\hline 34 & 209 & + & - & - & - \\
\hline 35 & 218 & + & - & - & - \\
\hline 36 & 221 & - & - & - & - \\
\hline 37 & 227 & + & - & - & - \\
\hline 38 & 231 & + & + & - & - \\
\hline
\end{tabular}




\begin{tabular}{|c|c|c|c|c|c|}
\hline No. & Terry No. & APDIs & ABVIs & PAs & GIs \\
\hline 39 & 237 & - & - & - & - \\
\hline 40 & 243R & - & - & - & - \\
\hline 41 & 247R & - & - & - & - \\
\hline 42 & 249R & - & - & - & - \\
\hline 43 & 259 & + & - & - & - \\
\hline 44 & 268 & + & - & - & - \\
\hline 45 & 272 & + & - & + & + \\
\hline 46 & 285 & - & - & - & - \\
\hline 47 & 293R & - & - & - & - \\
\hline 48 & 296R & + & - & - & - \\
\hline 49 & 298 & - & - & - & - \\
\hline 50 & 306R & - & - & - & - \\
\hline 51 & 314 & + & - & - & - \\
\hline 52 & 317 & - & - & - & - \\
\hline 53 & 338 & - & - & - & - \\
\hline 54 & 339R & + & - & - & - \\
\hline 55 & 344R & - & - & - & - \\
\hline 56 & 347 & - & - & - & - \\
\hline 57 & 348R & + & - & - & - \\
\hline 58 & 393RR & - & - & - & - \\
\hline 59 & 397 & - & - & - & - \\
\hline 60 & 403 & + & - & - & - \\
\hline 61 & 422 & + & - & - & - \\
\hline 62 & 437R & + & - & - & - \\
\hline 63 & 438 & - & - & - & - \\
\hline 64 & 445 & + & - & - & - \\
\hline 65 & 447 & + & - & - & - \\
\hline 66 & 452 & - & - & - & - \\
\hline 67 & 453 & + & - & - & - \\
\hline 68 & 458 & - & - & - & - \\
\hline 69 & 463 & + & - & - & - \\
\hline 70 & 465 & + & - & - & + \\
\hline 71 & 470 & + & - & + & - \\
\hline 72 & 477 & - & + & - & - \\
\hline 73 & 483 & + & - & - & - \\
\hline 74 & 496 & + & - & - & - \\
\hline 75 & 497 & - & - & - & - \\
\hline 76 & 506 & + & - & - & + \\
\hline 77 & 512 & + & + & - & - \\
\hline 78 & 513RR & - & - & - & - \\
\hline
\end{tabular}




\begin{tabular}{|c|c|c|c|c|c|}
\hline No. & Terry No. & APDIs & ABVIs & PAs & GIs \\
\hline 79 & 527 & - & - & - & - \\
\hline 80 & 528 & - & - & - & - \\
\hline 81 & 534 & - & + & - & - \\
\hline 82 & 536 & + & - & + & - \\
\hline 83 & 545 & + & - & - & - \\
\hline 84 & 552 & - & - & - & - \\
\hline 85 & 573 & + & - & - & - \\
\hline 86 & 582 & - & - & - & - \\
\hline 87 & 586 & - & - & - & - \\
\hline 88 & 597 & + & - & - & - \\
\hline 89 & 602 & - & - & - & - \\
\hline 90 & 608 & - & - & - & - \\
\hline 91 & 617R & - & - & + & - \\
\hline 92 & 627R & - & - & - & - \\
\hline 93 & 629 & - & - & + & - \\
\hline 94 & 636 & + & - & - & - \\
\hline 95 & $657 R$ & + & - & - & - \\
\hline 96 & 686 & - & - & + & - \\
\hline 97 & 694 & + & - & - & - \\
\hline 98 & $702 R$ & - & - & - & - \\
\hline 99 & 726 & - & - & - & - \\
\hline 100 & 727 & - & - & - & - \\
\hline 101 & 759 & + & - & + & - \\
\hline 102 & 789 & - & - & - & - \\
\hline 103 & 795 & + & - & - & - \\
\hline 104 & 809R & - & - & - & - \\
\hline 105 & 823 & + & - & - & - \\
\hline 106 & 833R & + & - & - & - \\
\hline 107 & 834R & - & - & - & - \\
\hline 108 & 863 & - & - & - & - \\
\hline 109 & 891 & - & - & - & - \\
\hline 110 & 903R & - & - & - & - \\
\hline 111 & 919 & - & - & - & - \\
\hline 112 & 930R & - & - & - & - \\
\hline 113 & 934 & - & - & - & - \\
\hline 114 & 938 & - & - & - & - \\
\hline 115 & 941 & - & - & + & - \\
\hline 116 & 946 & + & - & + & - \\
\hline 117 & 948 & - & - & + & - \\
\hline 118 & 957R & - & - & - & - \\
\hline
\end{tabular}




\begin{tabular}{|c|c|c|c|c|c|}
\hline No. & Terry No. & APDIs & ABVIs & PAs & GIs \\
\hline 119 & 964 & - & - & + & - \\
\hline 120 & 968 & - & - & - & - \\
\hline 121 & 973 & + & - & - & - \\
\hline 122 & 1023 & + & - & - & - \\
\hline 123 & 1029R & - & - & - & - \\
\hline 124 & 1045 & - & - & - & - \\
\hline 125 & 1046 & + & - & - & - \\
\hline 126 & 1050 & - & - & - & - \\
\hline 127 & 1058 & - & - & - & - \\
\hline 128 & 1060 & - & - & - & - \\
\hline 129 & 1066R & - & + & - & - \\
\hline 130 & 1070 & - & - & - & - \\
\hline 131 & 1071 & - & - & - & - \\
\hline 132 & 1098 & - & - & - & - \\
\hline 133 & 1100RR & - & - & - & - \\
\hline 134 & $1102 R$ & - & - & - & - \\
\hline 135 & 1107 & - & - & - & - \\
\hline 136 & 1130R & + & - & - & - \\
\hline 137 & 1133RR & - & - & - & - \\
\hline 138 & 1134R & - & - & - & - \\
\hline 139 & 1137R & - & - & - & - \\
\hline 140 & 1138R & + & - & - & - \\
\hline 141 & 1140 & + & - & - & - \\
\hline 142 & 1163 & - & - & - & - \\
\hline 143 & 1182R & - & - & - & - \\
\hline 144 & 1186 & + & - & - & - \\
\hline 145 & 1192 & + & - & - & - \\
\hline 146 & $1204 R$ & - & + & - & - \\
\hline 147 & 1219 & - & - & - & - \\
\hline 148 & 1224 & + & - & + & - \\
\hline 149 & 1228 & + & - & - & - \\
\hline 150 & 1229 & + & - & - & - \\
\hline 151 & 1232 & - & - & - & - \\
\hline 152 & 1243R & - & + & + & - \\
\hline 153 & 1252 & - & - & - & - \\
\hline 154 & 1267 & - & - & - & - \\
\hline 155 & 1271 & - & + & - & - \\
\hline 156 & 1277 & - & - & - & - \\
\hline 157 & 1291 & + & - & - & - \\
\hline 158 & 1299R & - & - & - & - \\
\hline
\end{tabular}




\begin{tabular}{|c|c|c|c|c|c|}
\hline No. & Terry No. & APDIs & ABVIs & PAs & GIs \\
\hline 159 & 1310 & - & - & - & - \\
\hline 160 & 1342 & + & - & - & - \\
\hline 161 & 1343 & - & - & - & - \\
\hline 162 & 1347R & - & - & - & - \\
\hline 163 & 1353R & - & - & - & - \\
\hline 164 & 1368 & - & - & + & - \\
\hline 165 & 1375R & - & - & - & - \\
\hline 166 & 1376 & - & - & - & - \\
\hline 167 & 1378 & - & - & - & + \\
\hline 168 & 1387 & - & - & + & - \\
\hline 169 & 1405 & - & - & - & - \\
\hline 170 & 1411R & - & - & - & - \\
\hline 171 & 1416 & - & - & - & - \\
\hline 172 & 1417R & + & - & - & - \\
\hline 173 & 1435 & - & - & - & - \\
\hline 174 & 1439R & - & - & - & - \\
\hline 175 & 1444 & - & - & - & - \\
\hline 176 & 1467 & - & - & - & - \\
\hline 177 & 1495 & - & - & - & - \\
\hline 178 & $1502 R$ & - & - & - & - \\
\hline 179 & 1505R & + & - & - & - \\
\hline 180 & 1514 & - & - & - & - \\
\hline 181 & 1519 & - & - & + & - \\
\hline 182 & 1534 & - & - & - & - \\
\hline 183 & 1543 & - & - & - & - \\
\hline 184 & 1549 & - & - & - & - \\
\hline 185 & 1552 & - & - & - & - \\
\hline 186 & 1554 & - & - & - & - \\
\hline 187 & 1567 & - & - & - & - \\
\hline 188 & 1581 & - & - & - & - \\
\hline 189 & 1592 & - & - & - & - \\
\hline 190 & 1599 & + & - & - & - \\
\hline 191 & 1604 & - & - & + & - \\
\hline 192 & 1614 & + & - & - & - \\
\hline 193 & 1627 & - & - & - & - \\
\hline
\end{tabular}


Supplementary table 5: Individual data of specimens exhibiting at least one type of probable TBM-associated endocranial alterations regarding possible TB-related non-endocranial bony changes in the TB group $(\Sigma=181)(+=$ present; $-=$ not present $)$.

\begin{tabular}{|c|c|c|c|c|c|c|c|c|}
\hline No. & Terry No. & $\begin{array}{l}\text { PNBFs on the } \\
\text { visceral } \\
\text { costal surfaces }\end{array}$ & HPO & $\begin{array}{l}\text { Extra-spinal } \\
\text { osteomyelitis }\end{array}$ & $\begin{array}{l}\text { Extra-spinal } \\
\text { arthritis }\end{array}$ & $\begin{array}{c}\text { Vertebral } \\
\text { hypervascularisation }\end{array}$ & $\begin{array}{c}\text { Vertebral lytic } \\
\text { lesions } \\
\text { and/or arthritis }\end{array}$ & $\begin{array}{c}\text { Reactive new bone } \\
\text { formations indicative of } \\
\text { a cold abscess }\end{array}$ \\
\hline 1 & $13 R$ & + & - & - & - & + & - & - \\
\hline 2 & $23 R$ & + & - & - & - & - & - & - \\
\hline 3 & 30R & - & - & - & - & + & - & - \\
\hline 4 & 54 & - & - & - & - & - & - & + \\
\hline 5 & 84 & - & - & - & - & - & + & + \\
\hline 6 & 87R & - & - & - & - & - & - & - \\
\hline 7 & 89R & + & - & - & - & + & + & + \\
\hline 8 & 90 & + & - & - & - & + & - & - \\
\hline 9 & 95 & - & + & - & - & - & + & + \\
\hline 10 & 95R & + & - & - & - & + & - & - \\
\hline 11 & 128 & + & - & - & - & + & - & - \\
\hline 12 & 129 & + & - & + & - & + & + & + \\
\hline
\end{tabular}




\begin{tabular}{|c|c|c|c|c|c|c|c|c|}
\hline No. & Terry No. & $\begin{array}{l}\text { PNBFs on the } \\
\text { visceral } \\
\text { costal surfaces }\end{array}$ & HPO & $\begin{array}{l}\text { Extra-spinal } \\
\text { osteomyelitis }\end{array}$ & $\begin{array}{l}\text { Extra-spinal } \\
\text { arthritis }\end{array}$ & $\begin{array}{c}\text { Vertebral } \\
\text { hypervascularisation }\end{array}$ & $\begin{array}{l}\text { Vertebral lytic } \\
\text { lesions } \\
\text { and/or arthritis }\end{array}$ & $\begin{array}{c}\text { Reactive new bone } \\
\text { formations indicative of } \\
\text { a cold abscess }\end{array}$ \\
\hline 13 & 138 & + & - & - & - & - & - & + \\
\hline 14 & 139 & - & - & - & - & + & - & - \\
\hline 15 & $145 R$ & + & - & - & - & + & - & - \\
\hline 17 & 158R & + & + & - & - & - & - & - \\
\hline 18 & 182 & + & - & - & - & + & - & + \\
\hline 19 & 194 & - & - & - & - & - & - & - \\
\hline 22 & 207 & + & - & - & + & - & - & - \\
\hline 23 & 220 & - & - & - & - & - & - & - \\
\hline 24 & 222 & + & - & - & - & + & - & - \\
\hline 25 & 230 & + & - & - & - & - & - & - \\
\hline 26 & 232R & - & - & - & - & - & - & - \\
\hline
\end{tabular}




\begin{tabular}{|c|c|c|c|c|c|c|c|c|}
\hline No. & Terry No. & $\begin{array}{c}\text { PNBFs on the } \\
\text { visceral } \\
\text { costal surfaces }\end{array}$ & HPO & $\begin{array}{l}\text { Extra-spinal } \\
\text { osteomyelitis }\end{array}$ & $\begin{array}{l}\text { Extra-spinal } \\
\text { arthritis }\end{array}$ & $\begin{array}{c}\text { Vertebral } \\
\text { hypervascularisation }\end{array}$ & $\begin{array}{c}\text { Vertebral lytic } \\
\text { lesions } \\
\text { and/or arthritis }\end{array}$ & $\begin{array}{c}\text { Reactive new bone } \\
\text { formations indicative of } \\
\text { a cold abscess }\end{array}$ \\
\hline 27 & 235 & - & - & - & - & + & - & + \\
\hline 28 & 251 & + & - & - & - & - & - & - \\
\hline 29 & 254 & + & + & - & - & - & - & - \\
\hline 31 & 265 & + & - & - & - & + & + & + \\
\hline 32 & 267 & - & - & + & - & + & - & - \\
\hline 33 & 269 & + & - & - & - & + & - & + \\
\hline 36 & 280 & - & - & - & - & + & - & - \\
\hline 37 & 283R & - & - & - & - & + & - & - \\
\hline 38 & 304 & - & - & - & - & + & - & - \\
\hline 39 & 306 & + & + & - & - & + & - & - \\
\hline 40 & 329 & + & - & + & - & - & + & + \\
\hline
\end{tabular}









\begin{tabular}{|c|c|c|c|c|c|c|c|c|}
\hline No. & Terry No. & $\begin{array}{l}\text { PNBFs on the } \\
\text { visceral } \\
\text { costal surfaces }\end{array}$ & HPO & $\begin{array}{l}\text { Extra-spinal } \\
\text { osteomyelitis }\end{array}$ & $\begin{array}{l}\text { Extra-spinal } \\
\text { arthritis }\end{array}$ & $\begin{array}{c}\text { Vertebral } \\
\text { hypervascularisation }\end{array}$ & $\begin{array}{l}\text { Vertebral lytic } \\
\text { lesions } \\
\text { and/or arthritis }\end{array}$ & $\begin{array}{c}\text { Reactive new bone } \\
\text { formations indicative of } \\
\text { a cold abscess }\end{array}$ \\
\hline 55 & 562 & + & - & - & - & + & - & - \\
\hline 56 & 565 & + & - & - & - & + & - & - \\
\hline 57 & 566 & + & - & - & - & - & - & - \\
\hline 59 & 571 & - & - & - & - & + & - & - \\
\hline 60 & 572 & + & - & - & - & - & - & - \\
\hline 61 & 575 & + & - & - & - & + & - & - \\
\hline 64 & 592 & + & + & + & - & + & - & - \\
\hline 65 & 595 & - & - & - & - & + & - & - \\
\hline 66 & 620 & - & - & - & - & - & + & - \\
\hline 67 & $621 R$ & + & - & - & - & + & - & - \\
\hline 68 & 664 & + & - & - & - & + & - & - \\
\hline
\end{tabular}




\begin{tabular}{|c|c|c|c|c|c|c|c|c|}
\hline No. & Terry No. & $\begin{array}{l}\text { PNBFs on the } \\
\text { visceral } \\
\text { costal surfaces }\end{array}$ & HPO & $\begin{array}{l}\text { Extra-spinal } \\
\text { osteomyelitis }\end{array}$ & $\begin{array}{l}\text { Extra-spinal } \\
\text { arthritis }\end{array}$ & $\begin{array}{c}\text { Vertebral } \\
\text { hypervascularisation }\end{array}$ & $\begin{array}{c}\text { Vertebral lytic } \\
\text { lesions } \\
\text { and/or arthritis }\end{array}$ & $\begin{array}{c}\text { Reactive new bone } \\
\text { formations indicative of } \\
\text { a cold abscess }\end{array}$ \\
\hline 69 & 680 & - & - & - & - & - & - & - \\
\hline 70 & $728 R$ & - & - & - & - & + & - & - \\
\hline 71 & 739 & - & - & - & - & + & - & - \\
\hline 73 & 757 & - & - & - & - & - & - & - \\
\hline 74 & 771 & - & - & - & - & - & - & - \\
\hline 75 & 776 & - & - & - & + & + & - & - \\
\hline 78 & $820 R$ & + & - & - & - & - & - & - \\
\hline 79 & 822 & + & - & - & - & + & - & - \\
\hline 80 & 828 & + & + & - & - & - & - & - \\
\hline 81 & 844 & - & - & - & - & - & - & - \\
\hline 82 & 846 & + & - & - & - & + & - & - \\
\hline
\end{tabular}




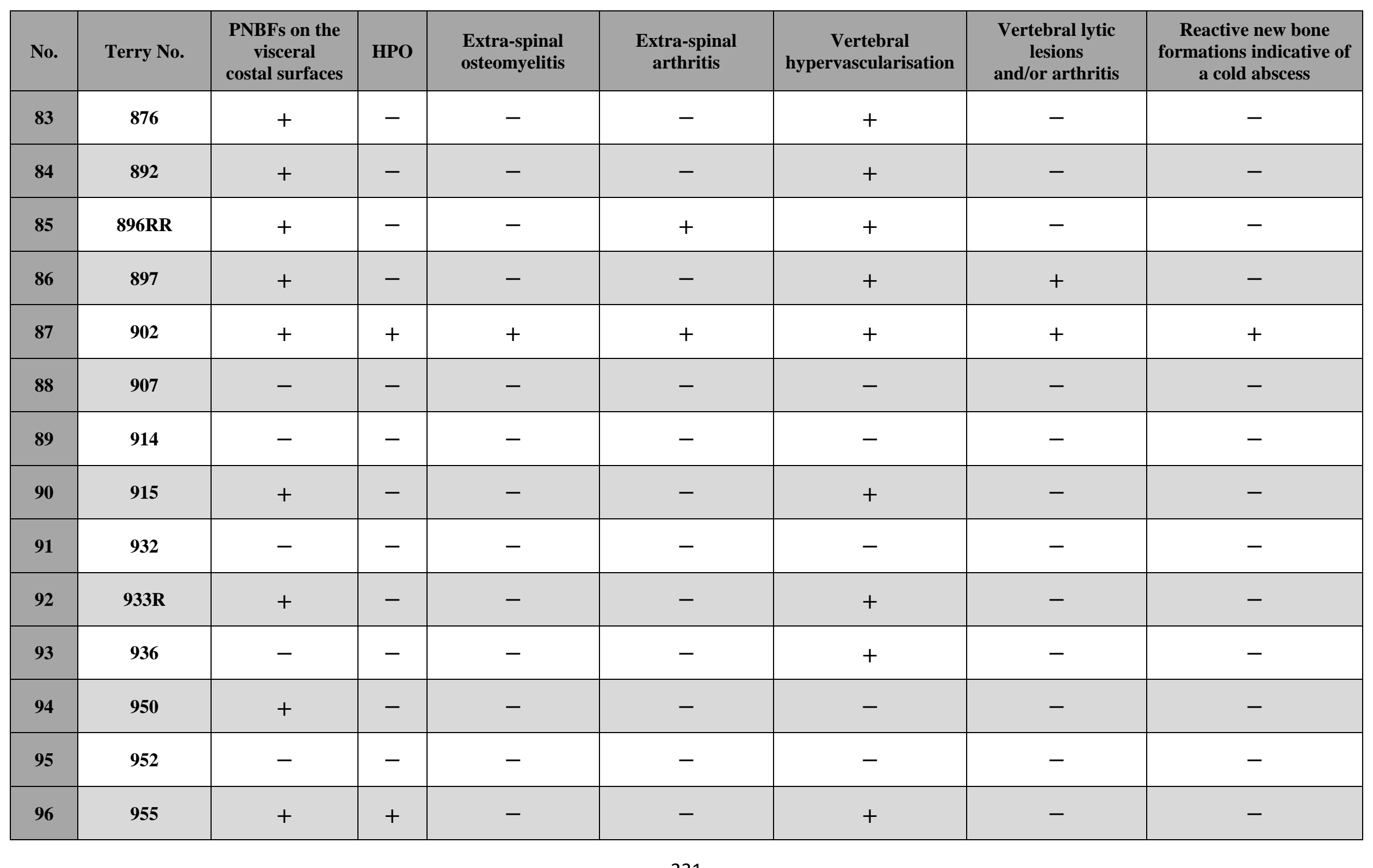



















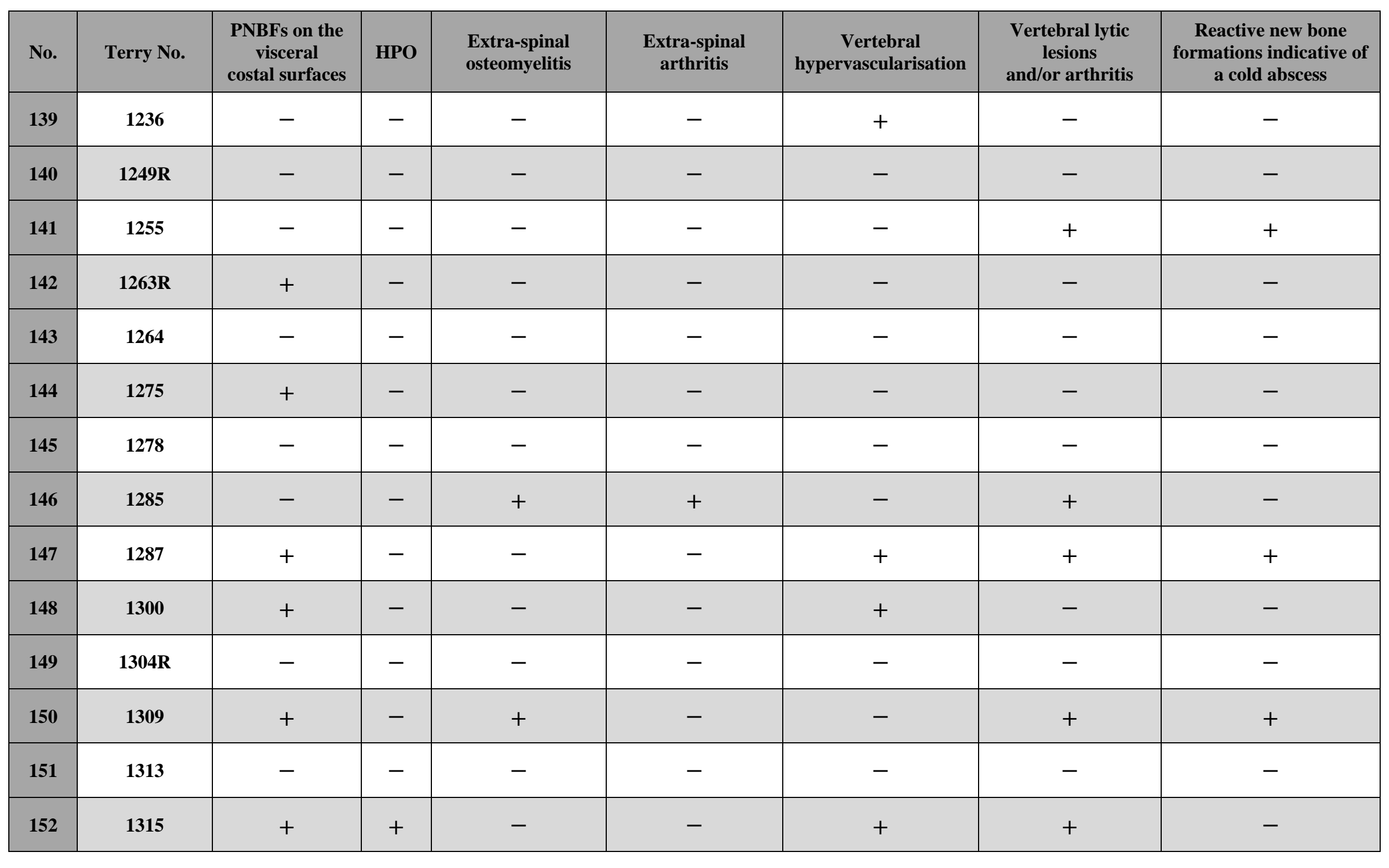














\begin{tabular}{|c|c|c|c|c|c|c|c|c|}
\hline No. & Terry No. & $\begin{array}{l}\text { PNBFs on the } \\
\text { visceral } \\
\text { costal surfaces }\end{array}$ & HPO & $\begin{array}{l}\text { Extra-spinal } \\
\text { osteomyelitis }\end{array}$ & $\begin{array}{l}\text { Extra-spinal } \\
\text { arthritis }\end{array}$ & $\begin{array}{c}\text { Vertebral } \\
\text { hypervascularisation }\end{array}$ & $\begin{array}{c}\text { Vertebral lytic } \\
\text { lesions } \\
\text { and/or arthritis }\end{array}$ & $\begin{array}{l}\text { Reactive new bone } \\
\text { formations indicative of } \\
\text { a cold abscess }\end{array}$ \\
\hline 180 & 1562 & - & - & - & - & - & - & - \\
\hline 181 & 1572 & + & - & - & - & - & - & - \\
\hline
\end{tabular}


Supplementary table 6: Individual data of specimens exhibiting at least one type of probable TBM-associated endocranial alterations regarding possible TB-related non-endocranial bony changes in the NTB group $(\Sigma=84)(+=$ present; $-=$ not present $)$.

\begin{tabular}{|c|c|c|c|c|c|c|c|c|}
\hline No. & Terry No. & $\begin{array}{l}\text { PNBFs on the } \\
\text { visceral } \\
\text { costal surfaces }\end{array}$ & HPO & $\begin{array}{l}\text { Extra-spinal } \\
\text { osteomyelitis }\end{array}$ & $\begin{array}{l}\text { Extra-spinal } \\
\text { arthritis }\end{array}$ & $\begin{array}{c}\text { Vertebral } \\
\text { hypervascularisation }\end{array}$ & $\begin{array}{c}\text { Vertebral lytic } \\
\text { lesions } \\
\text { and/or arthritis }\end{array}$ & $\begin{array}{c}\text { Reactive new bone } \\
\text { formations indicative of } \\
\text { a cold abscess }\end{array}$ \\
\hline 1 & $4 R$ & - & - & - & - & - & - & - \\
\hline 2 & $12 R$ & - & - & - & - & - & - & - \\
\hline 3 & $19 R$ & - & - & - & - & + & + & - \\
\hline 4 & $25 R$ & - & - & - & - & - & - & - \\
\hline 5 & $58 R$ & - & - & - & - & - & - & - \\
\hline 6 & 62RR & - & - & - & - & + & - & - \\
\hline 7 & $64 R$ & - & - & - & - & - & - & - \\
\hline 8 & $112 R$ & - & + & - & - & - & - & + \\
\hline 9 & 127R & - & - & - & - & - & - & - \\
\hline 10 & 135R & - & - & - & - & - & - & - \\
\hline 11 & 140RR & - & - & - & - & - & - & - \\
\hline 12 & 167 & - & - & - & - & - & - & - \\
\hline
\end{tabular}




\begin{tabular}{|c|c|c|c|c|c|c|c|c|}
\hline No. & Terry No. & $\begin{array}{l}\text { PNBFs on the } \\
\text { visceral } \\
\text { costal surfaces }\end{array}$ & HPO & $\begin{array}{l}\text { Extra-spinal } \\
\text { osteomyelitis }\end{array}$ & $\begin{array}{l}\text { Extra-spinal } \\
\text { arthritis }\end{array}$ & $\begin{array}{c}\text { Vertebral } \\
\text { hypervascularisation }\end{array}$ & $\begin{array}{l}\text { Vertebral lytic } \\
\text { lesions } \\
\text { and/or arthritis }\end{array}$ & $\begin{array}{c}\text { Reactive new bone } \\
\text { formations indicative of } \\
\text { a cold abscess }\end{array}$ \\
\hline 13 & 178R & - & - & - & - & - & - & - \\
\hline 14 & $197 R$ & - & - & - & - & - & - & - \\
\hline 15 & 209 & - & - & - & - & + & - & - \\
\hline 17 & 227 & - & - & - & - & - & - & - \\
\hline 18 & 231 & - & - & - & - & - & - & - \\
\hline 19 & 259 & - & - & - & - & + & - & - \\
\hline 22 & 296R & - & - & - & - & - & - & - \\
\hline 23 & 314 & - & - & - & - & - & - & - \\
\hline 24 & 339R & - & + & - & - & - & - & - \\
\hline 25 & 348R & - & - & - & - & - & - & - \\
\hline 26 & 403 & - & - & - & - & - & - & - \\
\hline
\end{tabular}



















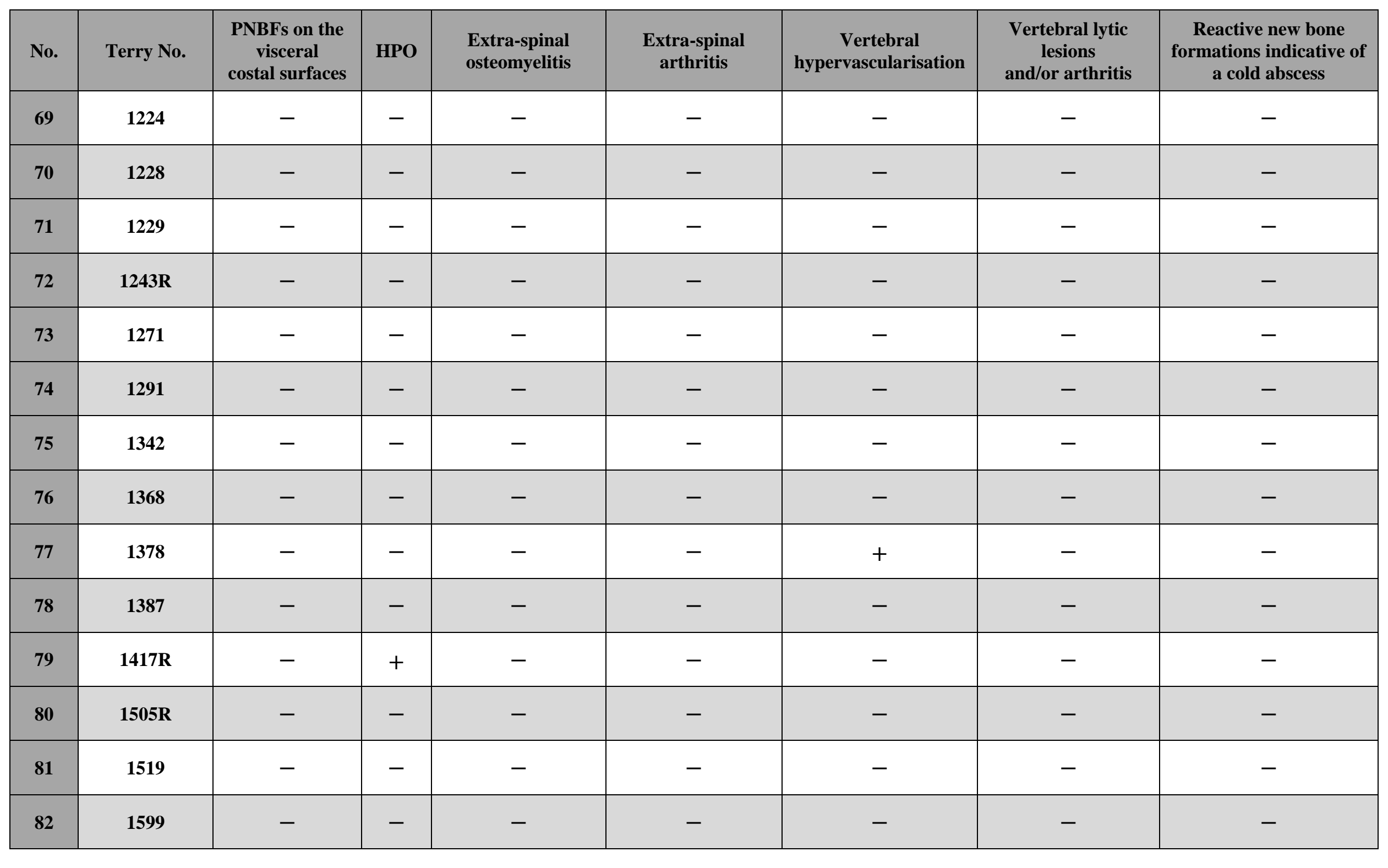




\begin{tabular}{|c|c|c|c|c|c|c|c|c|}
\hline No. & Terry No. & $\begin{array}{l}\text { PNBFs on the } \\
\text { visceral } \\
\text { costal surfaces }\end{array}$ & HPO & $\begin{array}{l}\text { Extra-spinal } \\
\text { osteomyelitis }\end{array}$ & $\begin{array}{l}\text { Extra-spinal } \\
\text { arthritis }\end{array}$ & $\begin{array}{c}\text { Vertebral } \\
\text { hypervascularisation }\end{array}$ & $\begin{array}{c}\text { Vertebral lytic } \\
\text { lesions } \\
\text { and/or arthritis }\end{array}$ & $\begin{array}{l}\text { Reactive new bone } \\
\text { formations indicative of } \\
\text { a cold abscess }\end{array}$ \\
\hline 83 & 1604 & - & - & - & - & - & - & - \\
\hline 84 & 1614 & - & - & - & - & + & - & + \\
\hline
\end{tabular}

